\title{
An enzyme-focused approach to create malic acid biosensors for winemaking
}

\section{Christopher Matthews}

A thesis submitted to the Victoria University of

Wellington in partial fulfilment of the requirements for the degree of Doctor of Philosophy

Victoria University of Wellington

(2022) 


\section{Abstract}

The tart-tasting malic acid is one of the key flavour determinants in wine. In winemaking, malic acid levels within grapes are carefully monitored prior to harvesting, as well as during secondary fermentations that aim to reduce its concentration. Commonly employed methods of measuring malic acid are expensive, consume large amounts of reagents, and require specialist laboratories and personnel to perform. Best exemplified by blood glucose monitors, enzyme-based amperometric biosensors are a solution to each of these problems, being easy to use, low-cost, and able to be integrated into miniature, portable devices. For measuring malate, these sensors use a malate oxidoreductase enzyme, which catalyses the oxidation of malate and concomitant reduction of a co-factor. When paired with an electrode, this electron transfer reaction can be transduced into a measurable electrical current.

Approximately 40 enzyme-based, amperometric malate sensors have been described in the peer-reviewed literature since the first described in 1980. Much of the work into improving devices has focused on the use of specialised materials or chemical additives to enhance electron transfer to the electrode. However, in general, little attention has been given to the choice of malate oxidoreductase used, with most devices using one of three commercially available enzymes. I hypothesised that the selection of a bespoke enzyme can be used to obtain desirable performance characteristics and afford novel utility in a biosensor. As the proof of principle, my choice of enzyme aimed to solve an issue that is particularly prevalent in the malate biosensing literature: the linear ranges of devices are poorly suited for the needs of winemakers. As these ranges are often extremely narrow, a winemaker must perform a careful and accurate dilution series to enable measurements. A device with a wider linear range would be more convenient for the end user.

Candidate malate oxidoreductases, characterised in the peer-reviewed literature, were selected based on their reported Michaelis-Menten parameters. I specifically focused on the Michaelis constant $\left(K_{\mathrm{M}}\right)$, a parameter that governs the hyperbolic relationship between increase in substrate concentration and enzyme saturation, theorising that an enzyme with a large $K_{\mathrm{M}}$ would yield a device with a wide linear range. Seven candidate enzymes were expressed, purified, and then characterised using spectrophotometric assays. The R181Q mutant of the malic enzyme from Ascaris suum was taken forward for the project. This 
enzyme has the highest $K_{\mathrm{M}}$ for malate of all characterised malate oxidoreductases and a mechanism to modulate the Michaelis-Menten parameters, including $K_{\mathrm{M}}$, by adding different concentrations of ammonium to assays.

An amperometric device was built using this enzyme in solution, on a gold screen-printed electrode. Six electron mediators were tested for their ability to increase the rate of electron transfer from the reduced co-factor, NADH, to the electrode surface. The aim was to produce the highest signal with the lowest operating potential, though it was ultimately found that many were incompatible with the sensor assay, being either insoluble, unstable, or the cause of electrode fouling. Conversely, 2,6-dichlorophenolindophenol proved compatible with the other assay components and gave relatively large signals at the relatively low potential of $+150 \mathrm{mV}$. The device created reports on malic acid concentrations up to $200 \mathrm{mM}$, the maximum concentration seen in grapes, and has the widest linear range of any enzyme-based malate biosensor described to date.

The addition of ammonium to assays was used to increase the sensitivity of the device. However, two other small molecules were also found that were capable of modulating the sensor response: $\mathrm{Mn}^{2+}$ and citrate. These three compounds, added in various combinations, were used to generate three different calibration curves. In this way, a novel type of biosensor was created with the previously undescribed utility of a linear range versus sensitivity tradeoff: the sensor could be dynamically made more sensitive if required, making it better at resolving small differences in malic acid, though each increase in sensitivity came with the reduction in the linear range. The device holds value in being operationally flexible to accommodate the needs of winemakers seeking to measure both large and small differences in malic acid.

The device was tested by applying a range of Pinot Noir and Pinot Gris grape juice taken from different stages of the winemaking process of grape and wine samples, with measurements being compared to two commercial malic acid testing kits. The sensor consistently produced current readings 5- to 6-fold higher than expected, based on the results of the commercial kits. This was shown to be due to grape juice and wine interfering with both the enzyme catalysed reaction and the mediator, resulting in non-specific current. Many of the compounds found within wine were tested for interfering effects, with ascorbic acid being identified as a potent interferent capable of reducing the mediator. Attempts to pre-treat 
real samples with chemical additives to remove ascorbic acid were unsuccessful, though an approach using an ascorbate oxidase remains to be tested.

This work represents a successful proof of the concept that careful selection of a bespoke enzyme can yield tremendous benefits to an amperometric biosensor, both in terms of the sensor performance and the addition of previously unseen utility. The presence of comprehensive databases, coupled with the current convenience of gene synthesis, suggests that the field of malate-sensing has much to gain from the search for new and better enzymes to be incorporated into devices. 


\section{Acknowledgments}

Of course, the greatest "thank you" goes to my supervisor, Wayne Patrick: one part academic, two parts agony aunt, your sage advice has got me through the $\mathrm{PhD}$ journey. Thanks for listening to my ideas and batting for my best interests. To both Wayne and Monica Gerth, thank you for the effort each of you has made to make the MME lab a fantastic place to work.

Each member of the MME lab (and our Ackerley lab cousins) deserves acknowledgment for their various technical support and for being superb company over the past three and a half years. I'd also like to thank Fraser Hughson for introducing me to the world of electrochemistry.

Thank you to my committee and to the members of the University's commercialisation team particularly Sam Wojcik and Matt Nicholson. I also greatly appreciate the help given by our partner winemakers, Maori Point, Hare's Run and Mount Difficulty Wines, who kindly provided the wine and grape juice samples I used for testing and gave their advice for creating a commercially successful device.

Lastly, my parents, for their unwavering support over the years. Thank you for the measured and balanced discussions, and for keeping me steady during my times of uncertainty. 


\section{Table of contents}

Chapter One - Introduction $\quad 1$

1.1. Foreword 1

1.2. The significance of malic acid 1

1.2.1. Malic acid in winemaking 3

1.3. Methods for quantifying malic acid 4

1.4. Introduction to the basic principles of enzyme-based amperometric biosensors 5

$\begin{array}{ll}\text { 1.5. Enzymes used for malate biosensors } & 7\end{array}$

$\begin{array}{ll}\text { 1.5.1. Malate dehydrogenase } & 7\end{array}$

1.5.2. Malic enzyme 9

1.5.3. Malate:quinone oxidoreductase 12

1.6. Sensitivity and the limit of detection 14

1.6.1. Background 14

1.6.2. Optimisation of enzymatic components 15

1.6.3. Optimisation of non-enzymatic electron transfer systems 18

1.7. Linear range $\quad 20$

1.8. Response time $\quad 22$

1.9. Long-term stability 23

1.10. Expanding the diversity of malate oxidoreductases used in biosensors 25

1.11. Summary and aims 27

Chapter Two - Screening candidate malate oxidoreductases $\quad 29$

2.1. Chapter introduction 29

2.2. Results $\quad 32$

2.2.1. Searching the BRENDA database for candidate malate oxidoreductases 32

2.2.2. Candidate enzyme profiles 34

2.2.2.1. AcME - Azorhizobium caulinodans, $\mathrm{NAD}^{+}$- dependent malic enzyme 35

2.2.2.2. AsME - Ascaris suum, $\mathrm{NAD}^{+}$- dependent malic enzyme 36

2.2.2.3. SVMDH - Sorghum vulgare, $\mathrm{NADP}^{+}$- dependent malate dehydrogenase 37

2.2.2.4. $\mathrm{ApMDH}$ - Aeropyrum pernix, $\mathrm{NAD}(\mathrm{P})^{+}$- dependent malate dehydrogenase $\quad 38$

2.2.2.5. TmME - Thermotoga maritima, putative malic enzyme 40

2.2.2.6. MaeA - Escherichia coli K12, NAD(P)+ malic enzyme 40

2.2.3. Cloning, expression and purification of chosen candidates 40 
2.2.4. Kinetic characterisation 47

$\begin{array}{ll}\text { 2.2.4.1. AcME } & 47\end{array}$

2.2.4.2. TmME 48

2.2.4.3. ApMDH and MaeA 50

2.2.4.4. AsME(R181Q) - the lead candidate 51

2.3. Discussion $\quad 55$

Chapter Three - Creating a working amperometric biosensor $\quad 59$

3.1. Chapter introduction $\quad 59$

3.1.1. Electron mediators $\quad 59$

3.1.2. Techniques used to study the electrochemistry of biosensors 60

3.1.2.1. Cyclic voltammetry 60

3.1.2.2. Chronoamperometry 61

3.2. Results 62

3.2.1. Screening electron mediators $\quad 62$

3.2.2. Creation of a standard assay and mediator optimisation 66

3.2.3. Biosensor \#1 - $\mathrm{Mg}^{2+}$ used as a co-factor 67

3.2.4. Biosensor \#2 - $\mathrm{Mn}^{2+}$ used as a co-factor 68

3.2.5. Biosensor \#3 - $\mathrm{Mn}^{2+}$ and citrate used together 69

3.2.6. Spectrophotometric assays using $\mathrm{Mn}^{2+}$ and citrate 72

3.2.7. Testing other activating compounds described in literature 73

$\begin{array}{ll}\text { 3.3. Discussion } & 73\end{array}$

3.3.1. The electrode 73

3.3.2. Selection of the mediator 74

3.3.3. Development of the sensor architecture $\quad 75$

3.3.4. Biosensor performance 76

Chapter Four - Applying real samples to the device $\quad 81$

$\begin{array}{ll}\text { 4.1. Chapter introduction } & 81\end{array}$

4.1.1. Compounds that commonly interfere with malate biosensors 82

4.1.2. Measurements in acidic samples 84

4.2. Results 85

4.2.1. Initial grape juice testing $\quad 85$

4.2.2. Development of a buffering system 86

4.2.3. Measuring malic acid across alcoholic fermentation 90 
4.2.4. Testing specific compounds found in wine for interference 94

4.2.4.1. Major organic compounds 94

4.2.4.2. Metal ions and amino acids 96

4.2.4.3. Phenolic compounds $\quad 97$

4.2.4.4. Ascorbic acid 98

$\begin{array}{ll}\text { 4.3. Discussion } & 100\end{array}$

$\begin{array}{ll}\text { 4.3.1. A buffering system for acidic samples } & 100\end{array}$

$\begin{array}{ll}\text { 4.3.2. Identifying the source of interference } & 101\end{array}$

$\begin{array}{ll}\text { Chapter Five - Summary, conclusions, and future work } & 104\end{array}$

$\begin{array}{ll}\text { 5.1. Summary and conclusions } & 104\end{array}$

5.2. Towards creating a useable sensor $\quad 107$

$\begin{array}{ll}\text { 5.3. General conclusions } & 108\end{array}$

$\begin{array}{lr}\text { Chapter Six- Materials and Methods } & 109\end{array}$

$\begin{array}{lr}\text { 6.1. Materials } & 109\end{array}$

$\begin{array}{ll}\text { 6.1.1. DNA } & 109\end{array}$

6.1.2. Bacterial strains 111

6.1.3. Supplements 111

6.2. DNA Manipulation 112

6.2.1. DNA electrophoresis 112

6.2.2. DNA purification 112

6.2.3. Plasmid extraction 113

6.2.4. Restriction cloning 113

$\begin{array}{ll}\text { 6.3. Microbiological techniques } & 114\end{array}$

$\begin{array}{ll}\text { 6.3.1. Preparation of starter cultures } & 114\end{array}$

6.3.2. Preparation of electrocompetent E. coli 115

6.3.3. Transformation of electrocompetent cells 115

6.3.4. Preparation of frozen stocks for long-term storage 116

6.4. Protein manipulation 116

$\begin{array}{ll}\text { 6.4.1. SDS-PAGE } & 116\end{array}$

$\begin{array}{ll}\text { 6.4.2. Protein expression } & 118\end{array}$

6.4.3. Co-expression with molecular chaperones 118

$\begin{array}{ll}\text { 6.4.4. Protein purification } & 118\end{array}$

6.4.4.1. Affinity chromatography using Ni-NTA resin and ÄKTA 119 
6.4.4.2. Affinity chromatography using TALON resin and gravity flow

6.4.4.3. Size-exclusion chromatography

6.4.4.4. Buffer exchange, concentrating, quantification and storage of purified proteins

6.7. Electrochemical techniques

6.7.1. Preparation of electrodes

6.7.2. Cyclic voltammetry

6.7.3. Chronoamperometry

Appendix 1. Chromatograms produced during size-exclusion chromatography for protein purification 


\section{List of abbreviations}

$\mathrm{A}_{280}$

$\mathrm{A}_{340}$

AcME

ApMDH

APS

AsME(R181Q)

BRENDA

CNT

DCPIP

$\mathrm{ddH}_{2} \mathrm{O}$

HAR

HEPES

IPTG

$\mathrm{kDa}$

LB

LOD

MaeA

$\mathrm{MDH}$

ME

MES

MLF
The absorbance at $280 \mathrm{~nm}$

The absorbance at $340 \mathrm{~nm}$

Azorhizobium caulinodans, $\mathrm{NAD}^{+}$- dependent malic enzyme

Aeropyrum pernix, $\mathrm{NAD}(\mathrm{P})^{+}$- dependent malate dehydrogenase

Ammonium persulfate

Ascaris suum, $\mathrm{NAD}^{+}$- dependent malic enzyme

Braunschweig Enzyme Database

Carbon nanotubes

2,6-dichlorophenolindophenol

Double distilled water

Hexaammineruthenium (III) chloride

4-(2-hydroxyethyl)-1-piperazineethanesulfonic acid

Isopropyl $\beta$-D-1-thiogalactopyranoside

Kilodalton

Lysogeny broth

Limit of detection

Escherichia coli $\mathrm{K} 12$, $\mathrm{NAD}(\mathrm{P})^{+}$malic enzyme

Malate dehydrogenase

Malic enzyme

2-(N-morpholino)ethanesulfonic acid

Malolactic fermentation 
MOPS

MQO

$\mathrm{NAD}^{+}$

NADH

$\mathrm{NADP}^{+}$

NADPH

Ni-NTA

$\mathrm{OD}_{600}$

PDB

Phyre2

SDS-PAGE

SvMDH

TAE

TEMED

TmME

Tris

$\mathrm{v} / \mathrm{v}$

$\mathrm{w} / \mathrm{v}$
3-(N-morpholino)propanesulfonic acid

Malate:quinone oxidoreductase

$\beta$-Nicotinamide adenine dinucleotide, oxidised form

$\beta$-Nicotinamide adenine dinucleotide, reduced form

$\beta$-Nicotinamide adenine dinucleotide 2'-phosphate, oxidised form

$\beta$-Nicotinamide adenine dinucleotide 2 '-phosphate, reduced form

Nickel-nitrilotriacetic acid

The optical density at $600 \mathrm{~nm}$

The Protein Data Bank

Protein homology/analogy recognition engine, version 2.0

Sodium dodecyl sulfate-polyacrylamide gel electrophoresis

Sorghum vulgare, $\mathrm{NADP}^{+}$- dependent malate dehydrogenase

Tris-acetate EDTA

$N, N, N^{\prime}, N^{\prime}$-Tetramethylethylenediamine

Thermotoga maritima, putative malic enzyme

Tris(hydroxymethyl)aminomethane

Volume/ volume

Weight/ volume 


\section{Chapter One - Introduction}

\subsection{Foreword}

The evaluation of the literature surrounding malic acid biosensors carried out for this project has been published as a review article in the journal Analytica Chimica Acta (Copyright Elsevier). With permission from Elsevier, this publication has been reproduced largely verbatim as the introduction to this thesis. Certain sections have been modified or expanded to improve the coherence, and have been marked in accordance with the Victoria University of Wellington submission guidelines.

The author contributions are as follows: Christopher Matthews (the author of this thesis) led the writing of the manuscript and the creation of the figures. An exception is Figure 4 from the paper, not included in this thesis, which was created by Emma Andrews. Writing was done under the supervision of Wayne Patrick, who helped shape the analysis and proof-read the manuscript. All authors provided critical feedback that helped structure the final submission.

Matthews, C. J., Andrews, E. S. V. and Patrick, W. M. (2021)

'Enzyme-based amperometric biosensors for malic acid - A review', Analytica Chimica Acta, 1156, pp. 338218

\subsection{The significance of malic acid}

Malic acid is the principal organic acid of many fruits, vegetables and their juices (Lobit et al., 2006). It is a popular additive (labelled E 296) for sweets and beverages, both to control $\mathrm{pH}$ and to confer a tart taste. It is also used as a buffer and chelator in household products such as lotions and cleansers, and it is a chemical building block for polymer synthesis. In 
addition to the naturally occurring L-enantiomer, the DL racemic mixture is also chemically synthesised from fossil resources. The size of the global market for DL-malic acid has recently been estimated at 60,000 - 200,000 tonnes per annum, with a corresponding market value of US\$105-350M (Kövilein et al., 2020). In many types of food manufacture, the concentration of the malate anion is diligently monitored. A product may taste flat if the concentration of malic acid is too low, or excessively sour if it is too high. Moreover, the increased acidity from malate can have important roles in altering the chemical stability of other flavour compounds, as well as preventing microbial spoilage. Quantitative measurements are key in carefully tuning the balance between taste and maintaining freshness (Volschenk et al., 2006).

It can also be useful to measure malate concentrations during cultivation, as it enables horticulturists to make time-critical harvesting decisions (Palma and Barroso, 2002). Shifting malate profiles during development have been reported for many fruits and vegetables (Volschenk et al., 2006, Ma et al., 2018, Moing et al., 2000, Shekhar and Iritani, 1979). For example, the malic acid concentration in potato tubers can fall from approximately $50 \mathrm{mM}$ to $15 \mathrm{mM}$ as the growing season progresses (Shekhar and Iritani, 1979). Similarly, malic acid content was shown to range from 1.7 to $29 \mathrm{mg} / \mathrm{g}$ fresh weight in 101 wild and cultivated apples, both between varieties and also depending upon the stage of fruit development (Ma $e t$ al., 2018).

Malic acid profiles within fruits have also been used to combat 'food fraud', which is the adulteration of food for economic gain. The most common targets for food fraud are fruit juices. In these, the ratio of malic acid to other flavour biomarkers can be used to screen for unlisted acidifying agents or sugars, juice-to-juice adulteration and for verifying geographical/cultivar origin (Bononi et al., 2016, Clausen et al., 2011, Ehling and Cole, 2011, Nikolaou et al., 2017). Tests such as these provide authorities with a method of quality assessment for imposing regulations and improving ingredient transparency (Dasenaki and Thomaidis, 2019). 


\subsubsection{Malic acid in winemaking ${ }^{1}$}

A particularly well-studied process, which has driven much of the research into malate biosensors, is winemaking. The international market for wine is very large, at over US $\$ 287 \mathrm{~B}$ in 2015 (Anderson et al., 2017). Foremost, malic acid has important roles in the flavour and mouthfeel of the finished wine, conferring crisp and refreshing flavour notes that balance excessive sweetness. As one of the predominant acids in grapes, the others being tartaric and citric acids, malic acid profoundly influences the $\mathrm{pH}$ of grape juice and wine, in turn influencing the development and stabilisation of other flavour compounds as well as those responsible for the colour and aroma (Volschenk et al., 2006).

A wide range of malic acid concentrations are relevant to the winemaking process. Starting on the vine, shifting malic acid profiles during grape growth can be used to monitor the maturation process of the grape and to make informed harvesting decisions. An initial growth phase is characterised by the accumulation of malic acid up to concentrations of approximately $200 \mathrm{mM}$. The subsequent onset of maturation, called veraison, marks a shift in grape metabolism that degrades malic acid through respiration, causing its concentration fall rapidly. The rate of this decrease is highly dependent on the temperature, being slower in cold environments and faster in hotter climates. Harvested grapes typically contain $30-50 \mathrm{mM}$ malic acid, though concentrations have been reported outside this range after particularly hot or cold harvesting seasons (Volschenk et al., 2006). Malic acid is also produced by the yeast Saccharomyces cerevisiae during alcoholic fermentation, with levels in grape juice increasing by up to $7.5 \mathrm{mM}$ depending on the strain and conditions used (Yéramian et al., 2007). However, particularly acidic grape juice ( $<\mathrm{pH} 2.9)$, often caused by high levels of malic acid, can prevent yeast growth, while $\mathrm{pH}$ values above 3.5 make the wine prone to microbial spoilage.

Various strategies are used to increase or decrease the malic acid concentration. Many of these are done to modulate the acidity of the wine. While $\mathrm{pH}$ can be changed through the use of additives, such a potassium bicarbonate, this method is often limited by laws preventing

\footnotetext{
1 'Malic acid in Winemaking' is a section that does not appear in my review article. Malic acid's role in winemaking is briefly discussed in the publication, though the topic has been expanded for this thesis.
} 
the addition of exogenous compounds to wine, or the potential to jeopardise a brand's image of being 'natural' and 'wholesome' (Volschenk et al., 2006). As a major acid in grapes, many winemakers seek to influence $\mathrm{pH}$ by instead changing the levels of malic acid. Such techniques include shading vines during veraison to slow the degradation through respiration, or conversely using trellises to increase the grapes' exposure to the sun (Morrison and Noble, 1990). Certain winemakers may choose to use grape cultivars that naturally produce less or more malic acid, such as Cabernet Sauvignon and Riesling varieties, respectively (Kliewer et al., 1967). However, malolactic fermentation (MLF) is the most common method of changing the malic acid content. This conversion uses various species of lactic acid bacteria, such as Oenococcus oeni, to convert the malic acid in the wine to lactic acid. The tartness is removed and is replaced by lactic acid's smoother and more full-bodied mouthfeel, as well as other flavours from by-products of the bacteria, such as the buttery flavour of diacetyl (Volschenk et al., 2006). MLF is commonly performed when producing red wines, though it is employed for certain white wines too, such as Chardonnay (Lasik-Kurdyś et al., 2018).

Using MLF, winemakers often seek the complete removal of malic acid, though this is often difficult to observe. Instead, concentrations less than $7.5 \mathrm{mM}$ are generally sufficient for the process to be deemed complete (Zoecklein et al., 1999, Sereni et al., 2020). The timing of MLF must be tightly controlled to avoid the spoilage of certain aromas and flavour, making it crucial to continually monitor malic acid levels to determine an endpoint for a conversion process that can occur over weeks or months (Volschenk et al., 2006).

\subsection{Methods for quantifying malic acid}

While there are important reasons for detecting and quantifying malic acid in the food and beverage industry, the most common approaches for measuring it are difficult (or even impossible) to implement in field and factory settings. High-performance liquid chromatography is commonly used, although other chromatographic, electrophoretic and spectrophotometric techniques such as gas chromatography and capillary electrophoresis have also been described. These have recently been reviewed in detail elsewhere (Sochorova et al., 2018). These analytical approaches share the need for a sizeable capital investment, specialised reagents, and a high level of technical expertise. Viticulturists, horticulturists and 
processers most commonly rely on outsourcing tests to dedicated laboratories - a process poorly suited for routine measurements or continuous monitoring. Simpler alternatives based on paper chromatography are available, but these are largely qualitative (Iland et al., 2013, Zoecklein et al., 1999).

There is an acknowledged need for inexpensive and easy-to-use devices that offer quantitative measurements in situ (Giménez-Gómez et al., 2017, Bucur et al., 2006, Molinero-Abad et al., 2014). Biosensors offer such traits, along with advantages of high selectivity, fast response times and real-time measurement. The flagship examples are the enzyme-based, electrochemical blood glucose biosensors for managing diabetes, which account for approximately $85 \%$ of the global biosensor market (Turner, 2013). The inherent advantages for electrochemical sensors in blood glucose monitoring - such as miniaturisation, ease of manufacture, and utility with complex biological fluids (Grieshaber et al., 2008) have also ensured that electrochemical sensors dominate the malate biosensing literature. Exceptions include an enzyme-based calorimetric sensor (Antonelli et al., 2008) and an engineered bacterial whole cell system, in which malate sensing is coupled to production of the green fluorescent protein (Ganesh et al., 2015). Moreover, of the electrochemical malic acid biosensors, the vast majority are amperometric (measuring current). Of the other measurement types, comparatively few potentiometric sensors have been published (Situmorang et al., 2001, Palleschi et al., 1994) and, to the best of my knowledge, impedimetric and conductimetric malic acid biosensors are yet to be described. This is perhaps not surprising, as amperometric devices are generally regarded as being more sensitive than potentiometric alternatives (Grieshaber et al., 2008). This literature review summarises the progress towards marketable malic acid biosensors and the limitations that remain to be overcome before they are likely to enter widespread use. Given their prevalence in the literature, and the advantages discussed above, the focus is solely on enzyme-based amperometric biosensors.

\subsection{Introduction to the basic principles of enzyme-based amperometric biosensors}

Amperometric sensors measure changes in electrical current; that is, the flow of electrons. Amperometry involves increases or decreases in signal as the result of oxidation or reduction 
reactions involving the analyte, driving a current response that corresponds to the analyte concentration. The signal is detected using an electrode, which is held at a chosen potential to facilitate the transfer of electrons. It is common for amperometric sensors to use enzymes to drive changes in current response, owing to their fast reaction velocities, high specificity, and ability to be immobilised. The analyte of interest is a substrate in the enzyme-catalysed redox reaction, and catalytic turnover results in the production (or depletion) of an electroactive agent. In turn, this electroactive agent may interact directly with the electrode surface to cause a change in current. More commonly, however, the electroactive product of the enzyme-catalysed reaction in turn reduces or oxidises an electron mediator, and it is this that interacts with the electrode. The most common reactions in enzyme-based amperometric biosensors either consume oxygen, produce hydrogen peroxide, or else produce one of the reduced, nicotinamide-derived cofactors NADH and NADPH (Prodromidis and Karayannis, 2002).

As detailed in section 1.5 below, three types of enzyme that use malate as a substrate have been used to construct biosensors. In each case, they catalyse the oxidation of malate, resulting in the transfer of electrons to an electron-accepting co-factor. For sensors using these malate oxidoreductase enzymes, the general scheme for generating an electrochemical response is shown in Figure 1.1. Broadly, the determinants of the current response can be sorted into two categories: 1 . the enzymatic production of the electroactive species; and 2. the rate at which the electroactive species is able to shuttle electrons to the electrode surface. The various components of a biosensor's architecture have profound effects on these processes. These components include the electrode material, the type and amount of enzyme used, the immobilisation matrix, and the choice of electron mediator. The general design principles and physicochemical effects of these components in enzyme-based biosensors have been reviewed elsewhere (Prodromidis and Karayannis, 2002, Nguyen et al., 2019, Kanyong et al., 2017). Here, particular emphasis is placed on reviewing the enzymes that have been used in malate biosensors, as the goal of this thesis is to demonstrate that this component is especially ripe for future development. Overall, I focus on how the architectures of malate biosensors contribute towards the performance characteristics that are crucial for creating commercially successful devices. 


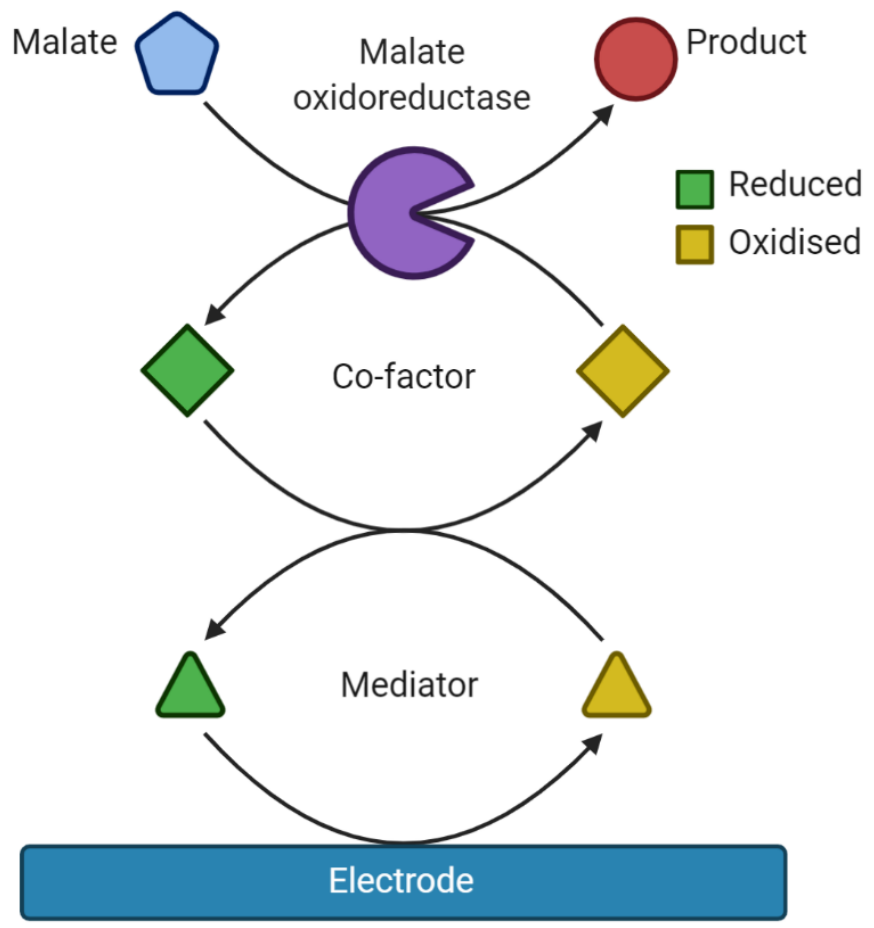

Figure 1.1. The stepwise transfer of electrons from malate to the electrode surface using a co-factordependent malate oxidoreductase enzyme. Most, but not all, malate biosensors also make use of a mediator to transfer electrons from the reduced co-factor to the electrode surface. Figure created with BioRender.com.

\subsection{Enzymes used for malate biosensors}

At least 34 distinct, enzyme-based amperometric biosensors for malate have been described in the peer-reviewed literature. These date back to the pioneering work of Blaedel and Engstrom in 1980. In this section, the different types of malate oxidoreductase enzyme that have been used to construct these biosensors are introduced. The published sensors are tabulated according to enzyme, along with some of their key functional parameters. Additional design features, such as the use of mono- versus bi-enzymatic systems, and key performance characteristics, including limits of detection, sensitivity, response time and longterm stability are discussed in more detail in the subsequent sections.

\subsubsection{Malate dehydrogenase}

By far the most commonly used enzyme for malate biosensors is malate dehydrogenase (MDH). It was used in 25 of the 34 biosensors I surveyed (Table 1.1), including all of the 
malate biosensors developed in the first 15 years of the field (Blaedel and Engstrom, 1980, Mizutani et al., 1991, Yoshioka et al., 1992, Silber et al., 1994, Cho et al., 1995). MDH reversibly catalyses the conversion of malate to oxaloacetate. Due to its crucial role in aerobic respiration, $\mathrm{MDH}$ is ubiquitous among prokaryotic and eukaryotic organisms (Goward and Nicholls, 1994, Minárik et al., 2002). The oxidation of malate can be accompanied by the reduction of $\mathrm{NAD}^{+}$to $\mathrm{NADH}$, or of $\mathrm{NADP}^{+}$to NADPH. Multiple isozymes are found in eukaryotes, with the mitochondrial and cytoplasmic forms being $\mathrm{NAD}^{+}$-dependent and the chloroplast isozyme in plants and algae using $\mathrm{NADP}^{+}$instead (Goward and Nicholls, 1994, Minárik et al., 2002). Co-factor preference is not always strict. For example, at $\mathrm{pH} 7.5$ the mitochondrial MDH from beef heart can use NADPH to reduce oxaloacetate at a rate $0.8 \%$ that observed with NADH (Englard and Siegel, 1969). Similarly, the $\mathrm{NAD}^{+}$-dependent enzyme from the bacterium Thermus flavus had detectable activity with oxaloacetate and NADPH, which could be increased more than 1000-fold by site-directed mutagenesis (Nishiyama et al., 1993). MDH enzymes with a preference for $\mathrm{NAD}^{+}$are classified as members of Enzyme Commission (EC) class 1.1.1.37, whereas the plant and algal enzymes with a preference for $\mathrm{NADP}^{+}$are members of $\mathrm{EC}$ 1.1.1.82. It is the $\mathrm{NAD}^{+}-$ preferring enzymes of EC 1.1.1.37 that have been used exclusively for malate biosensors (Giménez-Gómez et al., 2017, Blaedel and Engstrom, 1980, Mizutani et al., 1991, Yoshioka et al., 1992, Silber et al., 1994, Cho et al., 1995, Prodromidis et al., 1996, Gajovic et al., 1997, Katrlík et al., 1999, Miertuš et al., 1998, Maines et al., 2000, Manzoli et al., 2004, Gurban et al., 2006, Mazzei et al., 2007, Arvinte et al., 2008, Arvinte et al., 2009, Gamella et al., 2010, Monošík et al., 2012a, Vargas et al., 2016, Mundaca-Uribe et al., 2017, Röhlen et al., 2017). The reaction (equation (1)) involves a hydride transfer from malate to the co-factor (Cunningham et al., 1997). Under physiological conditions, the equilibrium position of this reaction lies far in the direction of malate formation, which becomes an important consideration for biosensor design (see section 1.6.2, below).

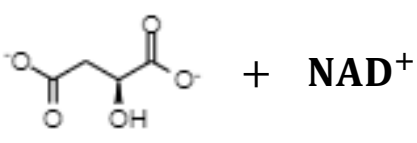

Malate

\section{Malate dehydrogenase}

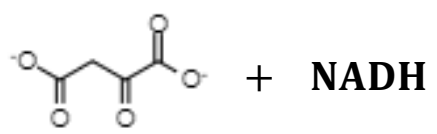

Oxaloacetate 


\subsubsection{Malic enzyme}

Malic enzyme (ME) catalyses the oxidative decarboxylation of malate, producing pyruvate and $\mathrm{CO}_{2}$ (equation (2)). Like MDHs, MEs can be either $\mathrm{NAD}^{+}$- dependent (EC 1.1.1.38) or $\mathrm{NADP}^{+}$-dependent (EC 1.1.1.40). MEs have been characterised from a wide range of animals, plants, fungi and prokaryotes, and because of their role in cellular lipid metabolism, they have been explored for biotechnological applications such as biofuel production (Liang and Jiang, 2015, Chang and Tong, 2003).

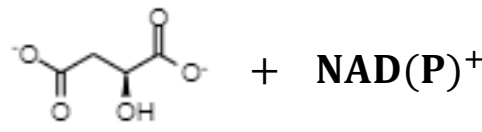

Malate

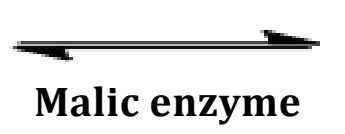

\section{Pyruvate}

Malic enzyme requires the presence of divalent metal ions (most commonly $\mathrm{Mg}^{2+} \mathrm{or}^{2+}$ ) to function (Liang and Jiang, 2015, Chang and Tong, 2003). Decarboxylation ensures that, unlike for $\mathrm{MDH}$, the forward reaction is highly favoured. Six publications have described ME-based amperometric malate biosensors (Table 1.2), starting with the work of Messia et al. in 1996. 
Table 1.1. Properties of amperometric malate biosensors that utilise the enzyme malate dehydrogenase (MDH), ordered by date of publication.

\begin{tabular}{|c|c|c|c|c|c|c|c|c|}
\hline Electrode $^{\mathrm{a}}$ & Enzyme(S) And Quantity & Mediator $^{c}$ & Enzyme Immobilisation Matrix ${ }^{d}$ & Real Samples & $\begin{array}{l}\text { LOD } \\
(\mu \mathrm{m})\end{array}$ & $\begin{array}{l}\text { Reported And } \\
\text { Normalised Sensitivities }\end{array}$ & $\begin{array}{l}\text { Linear } \\
\text { Range }(\mu \mathrm{m})\end{array}$ & Ref. \\
\hline Platinum & Porcine heart MDH (625 U) & None & $\begin{array}{l}\text { Acetylated molecular weight cut-off } \\
\text { membrane/NAD }\end{array}$ & None & 6 & $\begin{array}{l}0.2 \mu \mathrm{AmM}^{-1} \\
\left(0.16 \mu \mathrm{AM}^{-1} \mathrm{~cm}^{-2}\right)\end{array}$ & $6-400$ & (Blaedel and Engstrom, 1980) \\
\hline Clark & $\begin{array}{l}\text { Porcine heart MDH and Pediococcus sp. } \\
\text { NADH oxidase }\end{array}$ & None & $\begin{array}{l}\text { photocrosslinkable poly(vinyl alcohol) } \\
\text { bearing stilbazolium groups }\end{array}$ & $\begin{array}{l}\text { Apple, grape and fruit } \\
\text { juices; wine }\end{array}$ & 5 & $N R^{f}$ & $5-500$ & (Mizutani et al., 1991) \\
\hline Clark & $\begin{array}{l}\text { Porcine heart MDH }(240 \mathrm{U}) \text { and } \\
\text { Bacillus megaterium diaphorase }(60 \mathrm{U})\end{array}$ & Vitamin K & Cyanogen bromide-activated Sepharose & Red and white wine & $\mathrm{NR}^{f}$ & $N R^{f}$ & $50-1200$ & (Yoshioka et al., 1992) \\
\hline Gold & Porcine heart MDH $(3.8 \mathrm{U})$ & None & Poly(vinyl acetate)-polyethylene copolymer & None & 240 & $0.001 \mu \mathrm{AmM}^{-1}$ & $250-2000$ & (Silber et al., 1994) \\
\hline Carbon paste & Porcine heart MDH & PMS & $\begin{array}{l}\text { polyethylene glycol modified carbon } \\
\text { paste/NAD'/PMS }\end{array}$ & None & 500 & $N R^{f}$ & $500-10000$ & (Cho et al., 1995) \\
\hline Graphite & $\begin{array}{l}\text { Porcine heart mitochondrial MDH } \\
(7680 \mathrm{U} \text { ) and Clostridium kluyveri } \\
\text { diaphorase (23 U) }\end{array}$ & $\mathrm{HF}$ & Isothiocyanate controlled pore glass & $\begin{array}{l}\text { Peach, apple, grape, } \\
\text { kiwifruit, pear, beans }\end{array}$ & 1 & $N R^{f}$ & $20-400$ & (Prodromidis et al., 1996) \\
\hline Clark & $\begin{array}{l}\text { Porcine heart cytoplasmic MDH }(100 \mathrm{U} \mathrm{cm} \text { - } \\
\text { } 2) \text {, Pseudomonas sp. oxaloacetate } \\
\text { decarboxylase }\left(40 \mathrm{U} \mathrm{cm}^{-2}\right) \text { and Pediococcus } \\
\text { sp. pyruvate oxidase }\left(40 \cup \mathrm{cm}^{-2}\right)\end{array}$ & None & Gelatin/dialysis/poly(ethylene) membranes & $\begin{array}{l}\text { Red and white wine; } \\
\text { apple juice }\end{array}$ & 2 & $0.8 \mu \mathrm{AmM}^{-1} \mathrm{~cm}^{-2}$ & $N R^{f}$ & (Gajovic et al., 1997) \\
\hline $\begin{array}{l}\text { Graphite/HDO/ } \\
\mathrm{NAD}^{+}\end{array}$ & $\begin{array}{l}\text { Thermus flavus } \mathrm{MDH}(1.5 \mathrm{U}) \text { and } C . \text { kluyveri } \\
\text { diaphorase }(0.9 \mathrm{U})\end{array}$ & $\mathrm{HF}$ & Dried on electrode, dialysis membrane & Red, white and rosé wine & 10 & $N R^{f}$ & $10-1100$ & (Katrlik et al., 1999) \\
\hline $\begin{array}{l}\text { Graphite/SBM/ } \\
\mathrm{NAD}^{+}\end{array}$ & T. flavus MDH and C. kluyveri diaphorase & $\mathrm{HF}$ & Dried on electrode, dialysis membrane & Wines & 15 & $N R^{f}$ & $15-1500$ & (Miertuš et al., 1998) \\
\hline Platinum & $\begin{array}{l}\text { Porcine heart MDH and } C \text {. kluyveri } \\
\text { diaphorase }\end{array}$ & $\mathrm{HF}$ & $\begin{array}{l}\text { Cellulose acetate/cellulose nitrate/polyvinyl } \\
\text { chloride/polycarbonate/NAD } / \mathrm{HF}\end{array}$ & None & $N R^{f}$ & $N R^{f}$ & $\begin{array}{l}5000 \\
-30000\end{array}$ & (Maines et al., 2000) \\
\hline Graphite & Bovine heart MDH & None & $\begin{array}{l}\text { Glutaraldehyde/aminopropyl controlled } \\
\text { pore glass }\end{array}$ & $\begin{array}{l}\text { Orange, lime and } \\
\text { tangerine juices }\end{array}$ & $N R^{f}$ & $\begin{array}{l}1.35 \mu \mathrm{AmM}^{-1} \\
\left(0.69 \mu \mathrm{AM}^{-1} \mathrm{~cm}^{-2}\right)\end{array}$ & $5-100$ & (Manzoli et al., 2004) \\
\hline $\begin{array}{l}\text { Carbon paste/ } \\
\text { Prussian blue }\end{array}$ & $\begin{array}{l}\text { Porcine heart } \mathrm{MDH}(0.28 \mathrm{U}) \text { and Thermus } \\
\text { thermophilus } \mathrm{NADH} \text { oxidase }(0.05 \mathrm{U})\end{array}$ & $\begin{array}{l}\text { Prussian } \\
\text { blue }\end{array}$ & $\begin{array}{l}\text { photocrosslinkable poly(vinyl alcohol) } \\
\text { bearing stilbazolium groups }\end{array}$ & Red, white and rosé wine & 9.1 & $0.99 \mu \mathrm{AmM}^{-1}$ & $68-380$ & (Gurban et al., 2006) \\
\hline $\begin{array}{l}\text { Carbon paste/ } \\
\text { Meldola's blue }\end{array}$ & Porcine heart $\mathrm{MDH}(0.28 \mathrm{U})$ & $\begin{array}{l}\text { Meldola's } \\
\text { blue }\end{array}$ & $\begin{array}{l}\text { photocrosslinkable poly(vinyl alcohol) } \\
\text { bearing stilbazolium groups }\end{array}$ & Red, white and rosé wine & 9.4 & $1.09 \mu \mathrm{AmM}^{-1}$ & $94-797$ & (Gurban et al., 2006) \\
\hline Clark & T. flavus MDH and horseradish peroxidase & None & $\begin{array}{l}\text { Nylon membrane bearing carboxyl } \\
\text { groups/polyazetine/dialysis membrane }\end{array}$ & Red and white wine & 32 & $0.031 \mu \mathrm{A} \mathrm{mM} \mathrm{M}^{-1} \mathrm{~cm}^{-2}$ & $58-1700$ & (Mazzei et al., 2007) \\
\hline GC/SWCNT & Unspecified MDH (0.7 U) & None & Dried on electrode & None & 33 & $0.46 \mu \mathrm{Am}^{-1}$ & $200-800$ & (Arvinte et al., 2008) \\
\hline GC/SWCNT & Unspecified MDH (0.7 U) & None & Nafion & None & $N R^{f}$ & $0.2 \mu \mathrm{AmM}^{-1}$ & $200-1000$ & (Arvinte et al., 2008) \\
\hline $\begin{array}{l}\text { GC/SWCNT/ } \\
\text { Meldola's blue }\end{array}$ & Unspecified MDH (0.7 U) & $\begin{array}{l}\text { Meldola's } \\
\text { blue }\end{array}$ & Nafion & None & 3 & $12.4 \mu \mathrm{AmM}^{-1} \mathrm{~cm}^{-2}$ & $34-1350$ & (Arvinte et al., 2009) \\
\hline Gold/MPA & $\begin{array}{l}\text { T. flavus MDH }(4 \mathrm{U}) \text { and } C \text {. kluyveri } \\
\text { diaphorase }(1.6 \mathrm{U})\end{array}$ & TTF & $\begin{array}{l}\text { 3-mercaptopropionic acid self-assembled } \\
\text { monolayer/TTF/dialysis membrane }\end{array}$ & Synthetic wine & 0.52 & $\begin{array}{l}1.58 \mu \mathrm{AmM}^{-1} \\
\left(22.4 \mu \mathrm{Am}^{-1} \mathrm{~cm}^{-2}\right)\end{array}$ & $0.52-20$ & (Gamella et al., 2010) \\
\hline Gold & $\begin{array}{l}\text { T. flavus MDH }(1.5 \mathrm{U}) \text { and unspecified } \\
\text { diaphorase }(1 \mathrm{U})\end{array}$ & $\mathrm{HF}$ & Chitosan & Red and white wine & 5.41 & $\begin{array}{l}0.55 \mu \mathrm{AmM}^{-1} \\
\left(27.4 \mu \mathrm{Am}^{-1} \mathrm{~cm}^{-2}\right)\end{array}$ & $10-520$ & (Monošik et al., 2012a) \\
\hline $\begin{array}{l}\text { Gold/NE/ } \\
\text { MWCNT }\end{array}$ & $\begin{array}{l}\text { T. flavus MDH }(1.5 \mathrm{U}) \text { and unspecified } \\
\text { diaphorase }(1 \mathrm{U})\end{array}$ & $\mathrm{HF}$ & Chitosan & Red wine & 1.57 & $\begin{array}{l}1.17 \mu \mathrm{AmM}^{-1} \\
\left(58.2 \mu \mathrm{AM}^{-1} \mathrm{~cm}^{-2}\right)\end{array}$ & $10-610$ & (Monošik et al., 2012a) \\
\hline $\begin{array}{l}\text { Gold/NE/ } \\
\text { MWCNT* }\end{array}$ & $\begin{array}{l}\text { T. flavus MDH }(1.5 \mathrm{U}) \text { and unspecified } \\
\text { diaphorase }(1 \mathrm{U})\end{array}$ & $\mathrm{HF}$ & Chitosan & Red wine & 1.77 & $\begin{array}{l}1.22 \mu \mathrm{AmM}^{-1} \\
\left(60.7 \mu \mathrm{Am}^{-1} \mathrm{~cm}^{-2}\right)\end{array}$ & $10-610$ & (Monošik et al., 2012a) \\
\hline $\begin{array}{l}\text { Gold-sputtered } \\
\text { steel }\end{array}$ & $\begin{array}{l}\text { T. flavus MDH }(6 \mathrm{U}) \text { and } C \text {. kluyveri } \\
\text { diaphorase }(1.4 \mathrm{U})\end{array}$ & TTF & Dried on electrode/TTF/dialysis membrane & Red, white and rosé wine & 0.16 & $3.2 \mu \mathrm{A} \mathrm{mM}{ }^{-1}$ & $0.55-26.4$ & (Vargas et al., 2016) \\
\hline $\begin{array}{l}\text { Gold- } \\
\text { polypyrrole }\end{array}$ & $\begin{array}{l}\text { Porcine heart } \mathrm{MDH}(3 \mathrm{U}) \text { and } C \text {. kluyveri } \\
\text { diaphorase }(0.5 \mathrm{U})\end{array}$ & HAR & Polypyrrole/HAR & Red wine & 0.063 & $\begin{array}{l}21.8 \mu \mathrm{AmM} \mathrm{mM}^{-1} \\
\left(1350 \mu \mathrm{AM}^{-1} \mathrm{~cm}^{-2}\right)\end{array}$ & $0.1-1$ & (Giménez-Gómez et al., 2017) \\
\hline $\begin{array}{l}\text { StS/GC/MO/ } \\
\text { MWCNT/NAD }\end{array}$ & $\begin{array}{l}\text { Porcine heart MDH }(750 \mathrm{U}) \text { and unspecified } \\
\text { glutamate oxaloacetate transaminase }\end{array}$ & None & Entrapment within electrode materials & $\begin{array}{l}\text { Apricot, peach, apple, } \\
\text { orange and pear juices }\end{array}$ & 4.02 & $0.3 \mu \mathrm{AmM}^{-1}$ & $\begin{array}{l}2000- \\
50000\end{array}$ & (Mundaca-Uribe et al., 2017) \\
\hline Platinum & $\begin{array}{l}\text { Porcine heart MDH (1.5 U) and C. kluyveri } \\
\text { diaphorase }(2 \mathrm{U})\end{array}$ & $\mathrm{HF}$ & $\begin{array}{l}\text { Cross-linked via glutaraldehyde/bovine } \\
\text { serum albumin }\end{array}$ & None & 11 & $\begin{array}{l}0.7 \mu \mathrm{AmM} \mathrm{mM}^{-1} \\
\left(22.3 \mu \mathrm{mM}^{-1} \mathrm{~cm}^{-2}\right)\end{array}$ & $25-3000$ & (Röhlen et al., 2017) \\
\hline
\end{tabular}


${ }^{\mathrm{a}} \mathrm{HDO}=$ 2-hexadecanone; $\mathrm{NAD}^{+}=$nicotinamide adenine dinucleotide; $\mathrm{SBM}=$ solid binding matrix; $\mathrm{HEC}=$ hydroxyethyl-cellulose; $\mathrm{GC}=$ glassy carbon; $\mathrm{SWCNT}=$ single-walled carbon nanotubes; MPA = 3-mercaptopropionic acid; NE = N-eicosane; MWCNT = multi-walled carbon nanotubes; $\mathrm{MWCNT}^{*}=\mathrm{Nanocy}{ }^{\circledR} 7000$ multi-walled carbon nanotubes; StS = stainless steel; $\mathrm{MO}=$ mineral oil.

${ }^{b}$ The quantity of each immobilised enzyme in units $(U)$ is listed in cases where it was either reported, or could be calculated based on the information provided in the cited reference.

c $\mathrm{PMS}=$ phenazine methosulfate; $\mathrm{HF}=$ hexacyanoferrate(III); TTF = tetrathiafulvalene; HAR = hexaammineruthenium (III) chloride.

${ }^{\mathrm{d}} \mathrm{NAD}^{+}=$nicotinamide adenine dinucleotide; $\mathrm{PMS}=$ phenazine methosulfate; $\mathrm{HF}=$ hexacyanoferrate(III); TTF = tetrathiafulvalene, $\mathrm{HAR}=$ hexaammineruthenium (III) chloride.

e Some sensitivities were reported in $\mu \mathrm{A} \mathrm{mM}^{-1}$ whereas others were normalised for the area of the working electrode ( $\mu \mathrm{A} \mathrm{mM}^{-1} \mathrm{~cm}^{-1}$ ). Where possible, we have used information provided in each cited reference to calculate the areas of working electrodes, so that sensitivities can be normalised (in $\mu A \mathrm{mM}^{-1} \mathrm{~cm}^{-1}$ ) to allow direct comparisons between devices. These calculated estimates are listed in parentheses.

${ }^{\mathrm{f}} \mathrm{NR}=$ not reported.

Table 1.2. Properties of amperometric malate biosensors that utilise malic enzyme (ME), ordered by date of publication.

\begin{tabular}{|c|c|c|c|c|c|c|c|c|}
\hline Electrode & Enzyme(s) and quantity & Mediator $^{b}$ & Enzyme immobilisation matrix & Real samples & $\begin{array}{l}\text { LOD } \\
(\mu \mathrm{M})\end{array}$ & $\begin{array}{l}\text { Sensitivity } \\
\left(\mu \mathrm{A} \mathrm{mM}^{-1} \mathrm{~cm}^{-2}\right)\end{array}$ & $\begin{array}{l}\text { Linear range } \\
(\mu \mathrm{M})\end{array}$ & Ref. \\
\hline Platinum & $\begin{array}{l}\text { Chicken liver ME (2U) and Pediococcus sp. } \\
\text { pyruvate oxidase (3.1 U) }\end{array}$ & None & $\begin{array}{l}\text { Cellulose acetate/nylon immunoaffinity } \\
\text { membrane/polycarbonate }\end{array}$ & White wine, grape must & 0.5 & $\mathrm{NR}^{\mathrm{c}}$ & $1-500$ & (Messia et al., 1996) \\
\hline Clark & $\begin{array}{l}\text { Chicken liver ME }\left(20 \mathrm{U} \mathrm{cm}^{-2}\right) \text { and Pediococcus } \\
\text { sp. pyruvate oxidase }\left(40 \mathrm{U} \mathrm{cm}^{-2}\right)\end{array}$ & None & $\begin{array}{l}\text { Gelatin/dialysis/poly(ethylene) } \\
\text { membranes }\end{array}$ & $\begin{array}{l}\text { Red and white wine, } \\
\text { apple juice }\end{array}$ & 2 & 9.6 & $1-900$ & (Gajovic et al., 1997) \\
\hline Clark & $\begin{array}{l}\text { Chicken liver ME (5 U) and Escherichia coli } \\
\text { salicylate hydroxylase (3 U) }\end{array}$ & None & $\begin{array}{l}\text { Gelatin/dialysis/poly(ethylene) } \\
\text { membranes }\end{array}$ & $\begin{array}{l}\text { Wines, cider, apples, } \\
\text { apple juice }\end{array}$ & $n / a$ & 18.5 & $10-1200$ & (Gajovic et al., 1998) \\
\hline Carbon/Rhodium & Chicken liver ME $(3.8$ 国 10 10 & None & Dried on electrode & Apple, potato, tomato & 28 & $\mathrm{NR}^{c}$ & $28-700$ & (Arif et al., 2002) \\
\hline Platinum & Unspecified ME & PMS & Glutaraldehyde/aminopropyl glass beads & Red wine & 3 & $\mathrm{NR}^{\mathrm{c}}$ & $10-400$ & (Esti et al., 2004) \\
\hline Graphite/Meldola's blue & Unspecified ME (0.6U) & MB & $\begin{array}{l}\text { Polyethylenimine-glutaraldehyde cross- } \\
\text { linking membrane }\end{array}$ & Red and white wine & 10 & $\mathrm{NR}^{\mathrm{c}}$ & $10-1000$ & (Lupu et al., 2004) \\
\hline
\end{tabular}

${ }^{a}$ The quantity of each immobilised enzyme in units $(U)$ is listed in cases where it was either reported, or could be calculated based on the information provided in the cited reference.

${ }^{b}$ PMS = phenazine methosulfate; $\mathrm{MB}=$ Meldola's blue.

${ }^{\mathrm{c}} \mathrm{NR}=$ not reported. 


\subsubsection{Malate:quinone oxidoreductase}

Malate:quinone oxidoreductase (MQO, EC 1.1.5.4, reclassified from EC 1.1.99.16) is a membrane-associated flavoprotein that was first characterised in Corynebacterium glutamicum and has subsequently been identified in other bacteria including Escherichia coli and Helicobacter pylori (Molenaar et al., 1998, Kather et al., 2000). Like MDH, MQO catalyses the oxidation of malate to oxaloacetate, although its physiological role is to donate electrons to the quinones of the electron transport chain, rather than to $\mathrm{NAD}(\mathrm{P})^{+}$(equation (3)). Usefully, the forward reaction is highly favoured. The standard free energy difference for MQO-catalysed malate oxidation is $\Delta \mathrm{G}^{\circ \prime}=-18.5 \mathrm{~kJ} \mathrm{~mol}^{-1}$ (with menaquinone as the electron acceptor), compared with $\Delta \mathrm{G}^{\circ \prime}=+28.5 \mathrm{~kJ} \mathrm{~mol}^{-1}$ for the MDH-catalysed reaction (Kather et al., 2000).

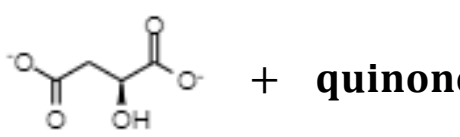

Malate

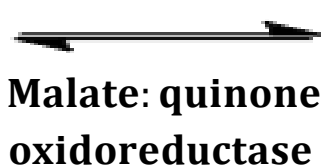
oxidoreductase

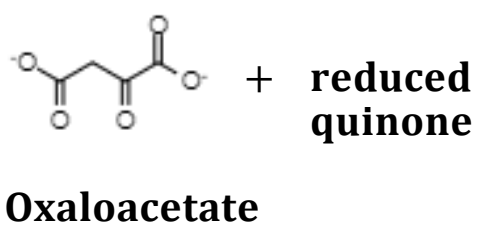

A potential advantage for MQO in malate biosensors is that its redox active co-factor is a tightly bound flavin adenine dinucleotide (FAD), rather than freely diffusing NAD(P) ${ }^{+}$ (Kather et al., 2000). The reduced form of this co-factor $\left(\mathrm{FADH}_{2}\right)$ can donate electrons directly to a variety of mediators (Bucur et al., 2006, Molinero-Abad et al., 2014). Three MQO-based amperometric biosensors have been described to date (Table 1.3). 
Table 1.3. Properties of amperometric malate biosensors that utilise malate:quinone oxidoreductase (MQO), ordered by date of publication.

\begin{tabular}{|c|c|c|c|c|c|c|c|c|}
\hline Electrode $^{a}$ & Enzyme and quantity & Mediator $^{c}$ & Enzyme immobilisation matrix & Real samples & $\operatorname{LOD}(\mu \mathrm{M})$ & $\begin{array}{l}\text { Reported and normalised } \\
\text { sensitivities }^{d}\end{array}$ & $\begin{array}{l}\text { Linear range } \\
(\mu \mathrm{M})\end{array}$ & Ref. \\
\hline Gr/HEC/TCNQ & Unspecified MQO & PMS & $\begin{array}{l}\text { Photocrosslinkable poly(vinyl alcohol) bearing } \\
\text { stilbazolium groups }\end{array}$ & Red and white wine & 5 & $\begin{array}{l}1.7 \mu \mathrm{AmM}^{-1} \\
\left(13.5 \mu \mathrm{AmM} \mathrm{mM}^{-1} \mathrm{~cm}^{-2}\right)\end{array}$ & $5-150$ & (Bucur et al., 2006) \\
\hline $\mathrm{Gr} / \mathrm{HEC} / \mathrm{TCNQ}$ & Unspecified MQO & DCPIP & $\begin{array}{l}\text { Photocrosslinkable poly(vinyl alcohol) bearing } \\
\text { stilbazolium groups }\end{array}$ & Red and white wine & 5 & $\begin{array}{l}0.85 \mu \mathrm{AmM}^{-1} \\
\left(6.8 \mu \mathrm{mM}^{-1} \mathrm{~cm}^{-2}\right)\end{array}$ & $5-250$ & (Bucur et al., 2006) \\
\hline C/AunP & Unspecified MQO $\left(2.8 \mathrm{U}^{\mathrm{b}}\right)$ & TTF & $\begin{array}{l}\text { Glutaraldehyde/bovine serum } \\
\text { albumin/tetrathiafulvalene }\end{array}$ & Red, white and rosé wine & 2 & $\begin{array}{l}5.75 \mu \mathrm{AmM}^{-1} \\
\left(46 \mu \mathrm{AM}^{-1} \mathrm{~cm}^{-2}\right)\end{array}$ & $2-19.6$ & (Molinero-Abad et al., 2014) \\
\hline
\end{tabular}

${ }^{a} \mathrm{Gr} / \mathrm{HEC} / \mathrm{TCNQ}=$ graphite/hydroxyethyl-cellulose/7,7,8,8-Tetracyanoquinodimethane (Reinecke salt); C/AuNP = carbon/gold nanoparticles.

${ }^{b}$ The quantity of immobilised enzyme in units $(U)$ was calculated based on the information provided in the cited reference.

${ }^{c}$ PMS = phenazine methosulfate; DCPIP = dichlorophenolindophenol; TTF = tetrathiafulvalene.

d Sensitivities were reported in $\mu \mathrm{A} \mathrm{mM}^{-1}$. We have used the information provided in each cited reference to calculate the area of the working electrode, so that sensitivities can be normalised (in $\mu \mathrm{A} \mathrm{mM}^{-1} \mathrm{~cm}^{-1}$ ) to allow direct comparisons between devices. These calculated estimates are listed in parentheses. 


\subsection{Sensitivity and the limit of detection}

\subsubsection{Background}

Two important considerations in designing and assessing the utility of malate biosensors are the limit of detection (LOD) and the sensitivity. The LOD is the smallest detectable change in analyte concentration. It is measured as the concentration of analyte that can be distinguished from the noise, typically defined as 2-3 times the standard deviation of the blank. As a product of the signal-to-noise ratio, LOD can be improved by decreasing the interferences that contribute to background noise, or by amplifying the signal that is specific to the electroactive analyte. The latter is quantified by a sensor's sensitivity, which describes the rate of change of current signal as the analyte concentration is increased; that is, sensitivity is the slope of the calibration curve.

In general, the stated motivation for new biosensor designs is to create a device with the best possible sensitivity (Gajovic et al., 1997, Maines et al., 2000, Arif et al., 2002). The sensitivities of enzyme-based amperometric biosensors are the result of a complex interplay between the enzyme, electron mediator, electrode, electrolyte and external variables (Borgmann et al., 2012). As highlighted in Tables 1.1-1.3, highly diverse combinations of these factors have been explored for malate biosensors. In turn, this makes it difficult to deconvolute and compare the key factors contributing to sensitivity. For example, most

publications report device sensitivity as the current generated per unit concentration of malate (e.g. units of $\mu \mathrm{A} \mathrm{mM}^{-1}$ ), rather than accounting for the area of the working electrode (e.g. $\mu \mathrm{A}$ $\mathrm{mM}^{-1} \mathrm{~cm}^{-2}$ ). Sensitivities were reported for 23 of the 34 biosensors we reviewed (Tables 1.11.3). Only five of these values were normalised for electrode area, although I was able to delve into methodological details in the cited references to estimate electrode areas (and thence normalised sensitivities) in 11 more cases. The difficulty in comparing normalised sensitivities is pervasive across the enzyme-based amperometric biosensor literature (Rathee et al., 2016, Rahman et al., 2010, Kanyong et al., 2017), impeding the most powerful direct comparisons of factors that contribute to sensitivity between different sensors.

Nevertheless, some trends are apparent and numerous strategies have been investigated for increasing sensitivity. Here, we broadly categorise these strategies as: (1) optimising the 
enzymatic components of the biosensor; and (2) optimising the non-enzymatic electron transfer systems. In the following sections we highlight selected examples and advancements.

\subsubsection{Optimisation of enzymatic components}

Most malate biosensors have used MDH as their enzymatic component (Table 1.1). However, as noted above (section 1.5.1.), the equilibrium of the MDH-catalysed reaction heavily favours the reverse reaction, which is the formation of malate rather than its oxidation (equation (1)). This makes it important to shift the equilibrium in favour of $\mathrm{NADH}$ formation. One strategy for this is to buffer the system at a mildly alkaline pH (Mizutani et al., 1991, Gajovic et al., 1997), which alters the protonation state of the enzyme to better accommodate malate/NAD ${ }^{+}$binding (Bernstein and Everse, 1978). However, the predominant strategy for improving the performance of MDH-based sensors has been to use a second enzyme for removing the reaction products and preventing the reverse reaction. NADH is most commonly targeted for enzymatic re-oxidation to $\mathrm{NAD}^{+}$(Mizutani et al., 1991, Yoshioka et al., 1992, Prodromidis et al., 1996, Katrlík et al., 1999, Miertuš et al., 1998, Maines et al., 2000), though, as will be described, oxaloacetate has also been targeted for removal (Gajovic et al., 1997, Mundaca-Uribe et al., 2017). Similar two-enzyme strategies have also been explored for ME-based sensors (Gajovic et al., 1997, Messia et al., 1996, Gajovic et al., 1998). All of the coupled enzymatic reactions that have been tested for use in malate biosensors are summarised in Table 1.4. 
Table 1.4. Enzymes that have been coupled to malate oxidoreductases in amperometric biosensors.

\begin{tabular}{ll} 
Enzyme and Catalysed Reaction & Reference \\
\hline & \\
$\mathrm{NADH}$ oxidase: & (Mizutani et al., 1991) $^{\mathrm{b}}$ \\
$\mathrm{NADH}+\mathrm{H}^{+}+\mathrm{O}_{2} \rightarrow \mathrm{NAD}^{+}+\mathrm{H}_{2} \mathrm{O}_{2}$ & $($ Gurban et al., 2006)
\end{tabular}

Oxaloacetate decarboxylase plus pyruvate oxidase:
oxaloacetate $\rightarrow$ pyruvate $+\mathrm{CO}_{2}$; and then
(Gajovic et al., 1997)
pyruvate $+\mathrm{PO}_{4}{ }^{2-}+\mathrm{O}_{2} \rightarrow$ acetylphosphate $+\mathrm{H}_{2} \mathrm{O}_{2}+\mathrm{CO}_{2}$
Pyruvate oxidase:
(Gajovic et al., 1997) ${ }^{\mathrm{b}}$
pyruvate $+\mathrm{PO}_{4}{ }^{2-}+\mathrm{O}_{2} \rightarrow$ acetylphosphate $+\mathrm{H}_{2} \mathrm{O}_{2}+\mathrm{CO}_{2}$
(Messia et al., 1996) ${ }^{c}$

Salicylate hydroxylase:

$\mathrm{NADPH}+$ salicylate $+\mathbf{O}_{\mathbf{2}} \rightarrow$ catechol $+\mathrm{CO}_{2}+\mathrm{NADP}^{+}$

(Gajovic et al., 1998)

(Yoshioka et al., 1992)

(Prodromidis et al., 1996)

(Katrlík et al., 1999)

(Miertuš et al., 1998)

Diaphorase:

(Maines et al., 2000)

$\mathrm{NAD}(\mathrm{P}) \mathrm{H}+$ mediator $\rightarrow \mathrm{NAD}(\mathrm{P})^{+}+$reduced mediator

(Gamella et al., 2010)

(Monošík et al., 2012a)

(Vargas et al., 2016)

(Giménez-Gómez et al., 2017)

(Röhlen et al., 2017)

Horseradish peroxidase:

$2 \mathrm{NADH}+\mathrm{O}_{2}+2 \mathrm{H}^{+} \rightarrow 2 \mathrm{NAD}^{+}+2 \mathrm{H}_{2} \mathrm{O}$

(Mazzei et al., 2007)

Glutamate oxaloacetate transaminase:

oxaloacetate + L-glutamate $\rightarrow$ L-aspartate +2 -

(Mundaca-Uribe et al., 2017)

oxoglutarate

\footnotetext{
a The component of the reaction that is measured is highlighted in bold typeface.

${ }^{b}$ Measured oxygen consumption.

${ }^{\mathrm{c}}$ Measured hydrogen peroxide production.
}

The first enzyme-based, amperometric malate biosensor, described in 1980, measured the $\mathrm{NADH}$ produced by MDH directly via a platinum electrode in the absence of a coupled enzymatic reaction (Blaedel and Engstrom, 1980). The biosensor used an electrode of approximately $1.3 \mathrm{~cm}^{2}$ and had a reported sensitivity of $0.2 \mu \mathrm{A} \mathrm{mM}^{-1}$, corresponding to a 
normalised sensitivity of approximately $0.16 \mu \mathrm{A} \mathrm{mM}^{-1} \mathrm{~cm}^{-2}$. The LOD of this pioneering biosensor was $6 \mu \mathrm{M}$. While the sensitivity of the device was relatively low (Table 1.1), it serves as a useful baseline for comparing with multi-enzyme sensors.

The first coupled enzyme sensor was reported in 1991 (Mizutani et al., 1991). It used NADH oxidase, both to recycle NADH back to $\mathrm{NAD}^{+}$, and also to allow oxygen consumption to be measured using a Clark electrode (Table 1.4): a platinum-based electrode with extraordinary specificity for oxygen due the use of a selectively permeable Teflon membrane (Clark and Lyons, 1962). While the LOD $(5 \mu \mathrm{M})$ was comparable to the previous biosensor, no estimate of sensitivity was provided. It was 1997 - 17 years after the original sensor - before the work of Gajovic et al. (1997) made it possible to assess the impact of a coupled enzyme system on sensitivity. These authors used a three-enzyme system: the oxaloacetate produced by MDH (equation (1)) was removed by oxaloacetate decarboxylase, which decarboxylated it to pyruvate. Then the pyruvate was oxidised with a pyruvate oxidase, enabling oxygen consumption to be measured (Table 1.4). Although the calibration curve was not linear, the estimated sensitivity was $0.8 \mu \mathrm{AmM}^{-1} \mathrm{~cm}^{-2}$ and the LOD was $2 \mu \mathrm{M}$, representing improvements of approximately 5-fold and 3-fold, respectively, compared to the sensor produced in 1980. However, the same study also emphasised that sensors based on ME, which oxidatively decarboxylates malate in one step (equation (2)), can be even more effective. Replacing MDH and oxaloacetate decarboxylase with ME (but keeping pyruvate oxidase to enable use of a Clark electrode) further improved sensitivity by over an order of magnitude, to $9.6 \mu \mathrm{A} \mathrm{mM}^{-1} \mathrm{~cm}^{-2}$ (Table 1.2) (Gajovic et al., 1997). This was despite the MEbased sensor functioning optimally at a lower enzyme loading of $20 \mathrm{U} \mathrm{cm}^{-2}$, compared to 100 $\mathrm{U} \mathrm{cm}^{-2}$ for the MDH-based sensor. Furthermore, in a subsequent study (Gajovic et al., 1998), the same authors paired ME with salicylate hydroxylase for NADPH recycling (Table 1.4), producing a sensor with even higher sensitivity $\left(18.5 \mu \mathrm{A} \mathrm{mM}^{-1} \mathrm{~cm}^{-2}\right)$. This work demonstrated that, like MDH-based systems, ME-based sensors can also benefit from approaches that deplete the reaction product(s) to drive malate oxidation.

After its use in the first coupled enzyme sensor (Mizutani et al., 1991), NADH oxidase has only been used in one subsequent study (Gurban et al., 2006). At first glance, NADH oxidase appears useful because it uses the dissolved oxygen in solution as the electron acceptor. However, the use of oxygen-based sensors has become less frequent as the field has progressed, presumably due to oxygen's poor interaction with certain electrode surfaces (Ucar et al., 2017), leading to less flexible sensor architectures, as well as unwanted 
fluctuations in baseline readings due to the sensitivity of dissolved oxygen concentration on environmental conditions (Gajovic et al., 1998). In contrast, the NADH/NADPH oxidising enzyme, diaphorase, has proven to be the most popular choice for co-factor recycling in malate biosensors (Table 1.4). It was used for 12 of the $25 \mathrm{MDH}$-based biosensors we reviewed. Diaphorase is capable of catalysing electron transfer from $\mathrm{NAD}(\mathrm{P}) \mathrm{H}$ to a wide range of oxidised mediators (Matsue et al., 1990), including the commonly used mediator hexacyanoferrate. This provides flexibility for designing and optimising the non-enzymatic components of the sensor. The well-characterised, commercially available and low-cost diaphorase from Clostridium kluyveri is a particularly favoured choice (Vargas et al., 2016, Prodromidis et al., 1996, Gamella et al., 2010, Giménez-Gómez et al., 2017, Katrlík et al., 1999, Maines et al., 2000, Miertuš et al., 1998, Monošík et al., 2012a, Röhlen et al., 2017).

It should be noted that, in addition to the pioneering malate biosensor, certain other malate sensors have opted to use an uncoupled malate oxidoreductase. Using multiple enzymes in a biosensor adds significant complexity to the design, as both must operate in the same conditions (i.e buffer, $\mathrm{pH}$ and the presence of reagents) despite potentially having different requirements for optimal activity. This adds to the time needed to develop the sensor and increases the cost of each device. The use of multiple enzymes can also decrease the selectivity as the substrates for each enzyme influences the reading. As will be discussed in section 1.8, multi-enzyme systems have been observed to have longer response times due to lag phases prior to reaching a steady state (Kucherenko et al., 2020).

\subsubsection{Optimisation of non-enzymatic electron transfer systems}

In 2002, Arif et al. showed it was possible to obtain an enhanced response in an ME-based malate biosensor by switching from a bare carbon electrode to rhodium-modified carbon. This marked a shift in focus away from developing new multi-enzyme systems towards improving sensitivity through the use of chemically modified electrodes. Subsequently, the variety of electrode materials and electron mediators explored in the literature expanded rapidly (Tables 1.1-1.3). Selection of the right mediator can result in sizeable improvements to both sensitivity and LOD due to fast shuttling of electrons from the reduced co-factor to the electrode surface, as well as rapid electron transfer upon arrival (Nagels and Staes, 2001). However, mediator performance is dependent on factors such $\mathrm{pH}$ (Gurban et al., 2006), immobilisation procedure (Giménez-Gómez et al., 2017) and electrode material (Ucar et al., 
2017), and accordingly, recent publications have screened to select the most suitable mediator before constructing the malate sensor itself (Lupu et al., 2004, Bucur et al., 2006).

At the turn of the millennium, the area of nanomaterials research experienced rapid growth (Bayda et al., 2019). In the creation of amperometric sensors, nanomaterials can be used to increase sensitivity by further promoting the rate of individual electron transfer reactions, as well as increasing the surface area of the electrode (Hayat et al., 2014). Accordingly, the use of electrocatalytic nanomaterials for malate biosensors has experienced a similar growth within the last two decades (Molinero-Abad et al., 2014, Gurban et al., 2006, Arvinte et al., 2008, Arvinte et al., 2009, Monošík et al., 2012a). The conductive properties of carbon nanotubes (CNTs) have ensured that they feature prominently in recent devices (Arvinte $e t$ al., 2008, Arvinte et al., 2009, Mundaca-Uribe et al., 2017). CNTs are cylindrical molecules comprising hexagonal arrangements of carbon atoms, which may be formed from rolling up a single sheet of graphene (giving a single-walled CNT) or by rolling up two or more sheets of graphene (giving a multi-walled CNT). Their positive impact on sensitivity was shown directly by Monosík et al. (2012a), who observed improvements of approximately 2-fold in sensitivity and 3-fold in the LOD for malate when a gold working electrode was modified with different multi-walled CNT composites (Table 1.1). The focus on modifying electrodes to increase sensitivity led to the creation of biosensors that dispensed with coupled enzymes and which also immobilised mediators on the electrode, rather than using them in solution (Arvinte et al., 2008, Arvinte et al., 2009, Arif et al., 2002). However, the devices with the overall highest sensitivities have combined the use of electrocatalytic electrode materials, electron mediators and a coupled diaphorase enzyme. For instance, a device that combined MDH and diaphorase with a hexaammineruthenium (III) chloride mediator and gold/polypyrrole electrode recorded a sensitivity of $1350 \mu \mathrm{A} \mathrm{mM}^{-1} \mathrm{~cm}^{-1}$, the highest described to date (Giménez-Gómez et al., 2017). 


\subsection{Linear range ${ }^{2}$}

The linear range of published malate biosensors is a particularly important topic, and is this focus of this thesis, as they are generally poorly suited for the needs of winemakers. A total of 16 publications test either wine or grape juice on their devices (Table 1.5). One describes the use of undiluted wine samples, though the others require at least a 10-fold dilution, with nine sensors requiring the sample to be diluted 100-fold or more. One device (Giménez-

Gómez et al., 2017), required red wine samples to be diluted up to 1 in 25,000. The need for a careful and accurate dilution series to reduce the malate concentration is undesirable for commercial products, as it requires a high level of technical competence that end-users may not have.

Table 1.5. Dilution of real samples required for published malate biosensors, ordered by date of publication.

\begin{tabular}{lll} 
Type of Real Sample & Dilution Required (fold) & Ref. \\
\hline Grape juice & $30-36^{a}$ & (Mizutani et al., 1991) \\
Wine & $35-40$ & (Yoshioka et al., 1992) \\
$\begin{array}{l}\text { Wine and grape must of maturing } \\
\text { grapes }\end{array}$ & $200-1000$ & (Messia et al., 1996) \\
Wine & 100 & (Gajovic et al., 1997) \\
Wine & 200 & (Gajovic et al., 1998) \\
Wine & 150 & (Katrlík et al., 1999) \\
Wine & $9-23^{a}$ & (Miertuš et al., 1998) \\
Malolactic fermentation & 100 & (Esti et al., 2004) \\
Wine & 100 & (Lupu et al., 2004) \\
Wine & 100 & (Bucur et al., 2006) \\
Wine & $17-51^{\text {a }}$ & (Gurban et al., 2006) \\
Wine & $8-24^{\mathrm{a}}$ & (Gurban et al., 2006) \\
Wine & $0-16^{\mathrm{a}}$ & (Mazzei et al., 2007) \\
Wine & Undiluted & (Monošík et al., 2012a) \\
Wine & $300-1000^{\text {a }}$ & (Molinero-Abad et al., 2014) \\
Wine & $5-10$ & (Vargas et al., 2016) \\
Wine & $10,000-25,000$ & (Giménez-Gómez et al., 2017) \\
\hline a The required dilution was not stated, and was calculated by dividing the determined \\
concentration of malate by the upper limits of the linear range.
\end{tabular}

\footnotetext{
${ }^{2}$ This section has been reworked from the peer-reviewed publication to include an expanded section on the dilution of wine samples. A discussion on the effect of Michaelis-Menten parameters on the linear range has been moved to the introduction of chapter 2 .
} 
While the trend in the field has been to build biosensors with ever-increasing sensitivity, my analysis revealed that this tends to trade off with linear range. Malate sensors exhibiting the highest sensitivities often have narrower linear ranges (Figure 1.2).

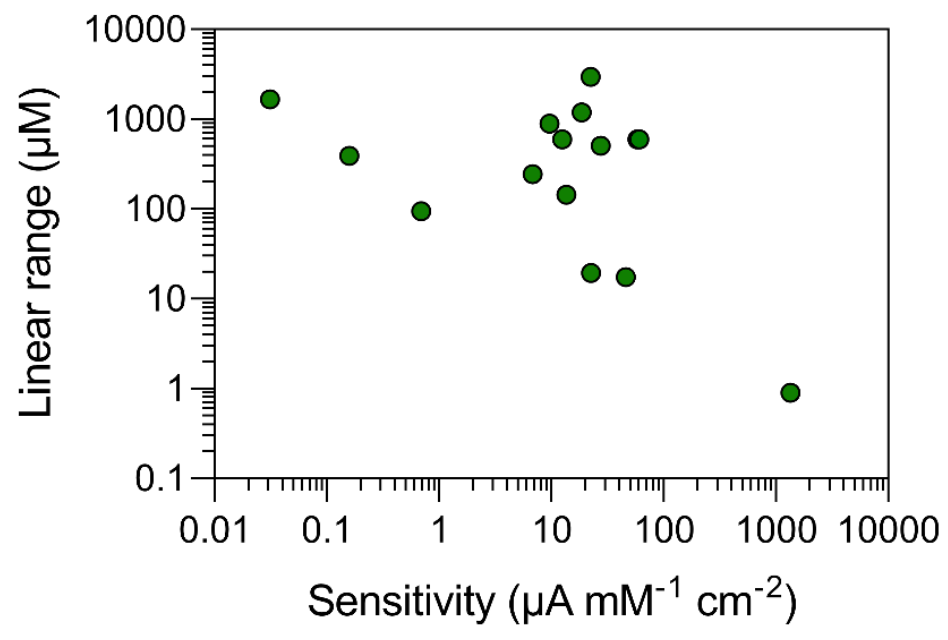

Figure 1.2. The trade-off between sensitivity and linear range for enzyme-based amperometric malate biosensors. To standardise the analysis, only sensors where the sensitivity was normalised for the area of the working electrode (reported or calculated in Tables 1.1-1.3) are included in the plot. Pearson's correlation = -0.2 .

The malate sensor that requires the greatest dilution of samples is almost the most sensitive (Giménez-Gómez et al., 2017). This device reported a sensitivity of $21.8 \mu \mathrm{A} \mathrm{mM}^{-1}$, which we estimate to be a normalised sensitivity of approximately $1350 \mu \mathrm{A} \mathrm{mM}^{-1} \mathrm{~cm}^{-2}$ (because the reported working electrode area was $1.62 \mathrm{~mm}^{2}$ ). This is at least 20 -fold more sensitive than any of the other biosensors we reviewed, though also has the narrowest linear range, $0.1-1$ $\mu \mathrm{M}$ malate. On the other hand, the second-most sensitive malate biosensor in the literature had a substantially lower normalised sensitivity (approximately $61 \mu \mathrm{A} \mathrm{mM}^{-1} \mathrm{~cm}^{-2}$ ), but displayed a linear response over the range 10-610 $\mu \mathrm{M}$ malate (Monošík et al., 2012a). This device could be used with undiluted wine samples, albeit with pre-treatment to remove interfering polyphenols.

Several factors govern the linear range of enzyme-based amperometric sensors (Silverstein and Goodney, 2010), making it difficult to discern the underlying cause of this relationship between linear range and sensitivity for each individual device. These factors include the 
immobilisation matrix, the diffusive properties of the mediator, and most importantly for this project, the Michaelis-Menten behaviour of the enzyme (further explained in Chapter 2) (Vasylieva and Marinesco, 2013, Thévenot et al., 2001). In terms of creating a commercially viable device, the ideal future biosensor is likely to be one that maximises linear range while retaining high sensitivity.

\subsection{Response time}

The response time of a sensor refers to the time taken to reach the final reading after the sample is applied, reflecting the time required to capture a detectable number of analytes on its surface. It is another important parameter in terms of ease-of-use and applicability. For instance, a sensor with a long response time may be unsuitable for rapid batch processing in industrial production lines, or for routine use by non-experts. Therefore, short response times are often desirable for practical applications. For example, the blood glucose sensors used by diabetics return a measurement within 5-15 s.

In general, response times for malic acid biosensors have not yet reached the gold standard of blood glucose monitors. The pioneering malate sensor from 1980 allowed for measurement in 5-11 min (Blaedel and Engstrom, 1980). This was rapidly improved to a response time of 1 min for the first bi-enzymatic sensor (Mizutani et al., 1991). However, there has been little advancement in response times since then. For example, the shift to chemically modified electrodes yielded a response time of $110 \mathrm{~s}$ (Arif et al., 2002) and the two most sensitive biosensors developed to date had response times of 70-120 s (Giménez-Gómez et al., 2017, Monošík et al., 2012a). Only two sensors, both from the same study (Arvinte et al., 2008), can be described as 'rapid': a response time of $8 \mathrm{~s}$ was reported for a device with adsorbed MDH on glassy carbon electrode modified with single-walled carbon nanotubes, while the response time was "less than $15 \mathrm{~s}$ " when the same enzyme was immobilised within a Nafion membrane instead.

Reducing the response times of malate biosensors is likely to be an important aspect of their commercialisation and real-world applicability. Generally, discussions on quickening response times are focused on improving the diffusion of analytes to the electrode surface, typically via the immobilisation procedure (Sassolas et al., 2012, Soleymani and Li, 2017). However, in keeping with the theme of this thesis, enzyme-related factors should not be 
overlooked. In general, multi-enzyme systems can suffer from increased response times because of the lag phase required for multiple sequential reactions to reach a linear steady state with a stable reaction rate (Kucherenko et al., 2020). However, this lag phase can be reduced by manipulating conditions (e.g. $\mathrm{pH}$; relative amounts of each enzyme; etc.) such that the malate oxidoreductase remains highly active, but the coupled enzyme is not rate limiting (Bisswanger, 2014). Accordingly, for enzyme-centric strategies that improve response time, designing sensors around a single enzyme, or using harmonious multi-enzyme systems, may be key.

\subsection{Long-term stability ${ }^{3}$}

The long-term storage stability, or 'shelf life', of a biosensor describes the retention of activity over time when stored under well-defined conditions. High storage stabilities are crucial for commercially successful sensors, which must retain activity despite the rigours of manufacturing, distribution and retail/consumer storage. In general, there is a minimum shelf life requirement of at least 6 months for sensors to be practical (Bhalla et al., 2016). As with other sensor types (Reyes-De-Corcuera et al., 2018), optimising the shelf life and storage conditions of enzyme-based amperometric malate biosensors is not generally the focus of the research literature. Of the reported stabilities, most (15 of 23) are three months or less. At the other extreme, two publications describe sensor stabilities of six months or more (Prodromidis et al., 1996, Monošík et al., 2012a).

For studies that immobilise $\mathrm{NAD}(\mathrm{P})^{+}$and/or the mediator, improvements to sensor lifetime involve the use of co-factor retaining membranes, or else screening mediators on the basis of their compatibility with the entrapment procedure (Giménez-Gómez et al., 2017, Bucur et al., 2006, Maines et al., 2000). Little focus has been placed on stabilising the enzyme. Numerous processes contribute to enzyme inactivation over time, including denaturation, proteolysis and irreversible inhibition, although various strategies can be employed to mitigate these effects (Gibson, 1999). Of these, the immobilisation procedure is particularly important, with the precise effects on stability and activity having been recently reviewed elsewhere (Reyes-

\footnotetext{
${ }^{3}$ The section 'Long-term stability' is preceded by another section in the publication: 'Interference'. This section has been used as the introduction to chapter 4.
} 
De-Corcuera et al., 2018). Many enzyme immobilisation procedures have been reported for malate biosensors, including adsorption (Maines et al., 2000, Arvinte et al., 2008, Vargas et al., 2016), entrapment (Silber et al., 1994, Gajovic et al., 1997, Gajovic et al., 1998), crosslinking (Molinero-Abad et al., 2014, Röhlen et al., 2017), covalent modification (Yoshioka et al., 1992, Messia et al., 1996, Esti et al., 2004), self-assembled monolayers (Gamella et al., 2010) and the use of anion repellent layers such as Nafion (Arvinte et al., 2008, Arvinte et al., 2009).

One study stands out for its emphasis on long-term stability. Monošík et al. set out to design a biosensor with a long shelf life, with the specific goal of incorporating it into a pre-existing commercial portable analytical device (Monošík et al., 2012a). They reported sensors based on three different electrodes (Table 1.1), each of which displayed a year-long shelf-life at room temperature. This outstanding long-term stability was attributed to the use of a chitosan 'sandwich' for enzyme stabilisation, and the storage of sensors in desiccators to avoid the negative impacts of humidity on activity (Monošík et al., 2012a). These sensors also made use of MDH from the thermophilic bacterium Thermus flavus, which is an enzyme naturally resistant to thermal inactivation (Kelly et al., 1993, Iijima et al., 1980). Thermostable enzymes are recognised as valuable tools across commercial biotechnology. Longer half-lives at ambient temperatures allow for more convenient and cost-effective means of preparing products, as well as offering resistance to other sources of activity loss, such as proteolysis or chemical-induced alterations to protein structure (Kambourova, 2018). In addition to the $T$. flavus enzyme, thermostable MDHs have also been characterised from other extremophilic microorganisms (Gharib et al., 2016, Wang et al., 2011). The utility of these other enzymes in malate biosensors remains to be tested. 


\subsection{Expanding the diversity of malate oxidoreductases used in biosensors ${ }^{4}$}

At the end of the 20th century, the emergence of protein engineering inspired a call for biosensor researchers to "adapt the molecule to the instrument, rather than the instrument to the molecule" (Hellinga and Marvin, 1998). The idea was to identify or to engineer proteins that were optimal for generic detector technologies, rather than optimising the non-protein components for use with off-the-shelf enzymes. Ironically, this was around the time that much of the malate biosensor literature was focused on advancing electrode design and immobilisation strategies, at the expense of focusing on the enzyme (section 1.6.3, above). In this section, we suggest that now is the time to explore more ambitious enzyme-based avenues for designing the next generation of malate biosensors. Further, much of what is discussed could also be applied to the broader field of enzyme-based amperometric biosensors.

Most noticeably, only a small number of commercially available malate oxidoreductase enzymes have been tested in biosensors. Of the $25 \mathrm{MDH}$-based sensors we reviewed (Table 1.1), 13 made use of porcine enzymes and eight used the thermostable MDH from $T$. flavus. One study used bovine MDH (Manzoli et al., 2004) and the other three did not specify the species origins of their commercially-sourced enzymes. The ME-based sensors (Table 1.2) either used commercial preparations of the chicken enzyme (four sensors), or enzymes of unspecified origin (two sensors). None of the MQO-based sensor studies specified the origins of their enzymes (Table 1.3). Thus, in the first four decades of the field, enzymes from only four named species have been explored for their utility in malate biosensors. This is visualised in Figure 1.3.

\footnotetext{
${ }^{4}$ The section 'Expanding the diversity of malate oxidoreductases used in biosensors' found in the peer-reviewed publication has been altered to remove the 'Protein engineering' subjection, which is not relevant for this thesis.
} 


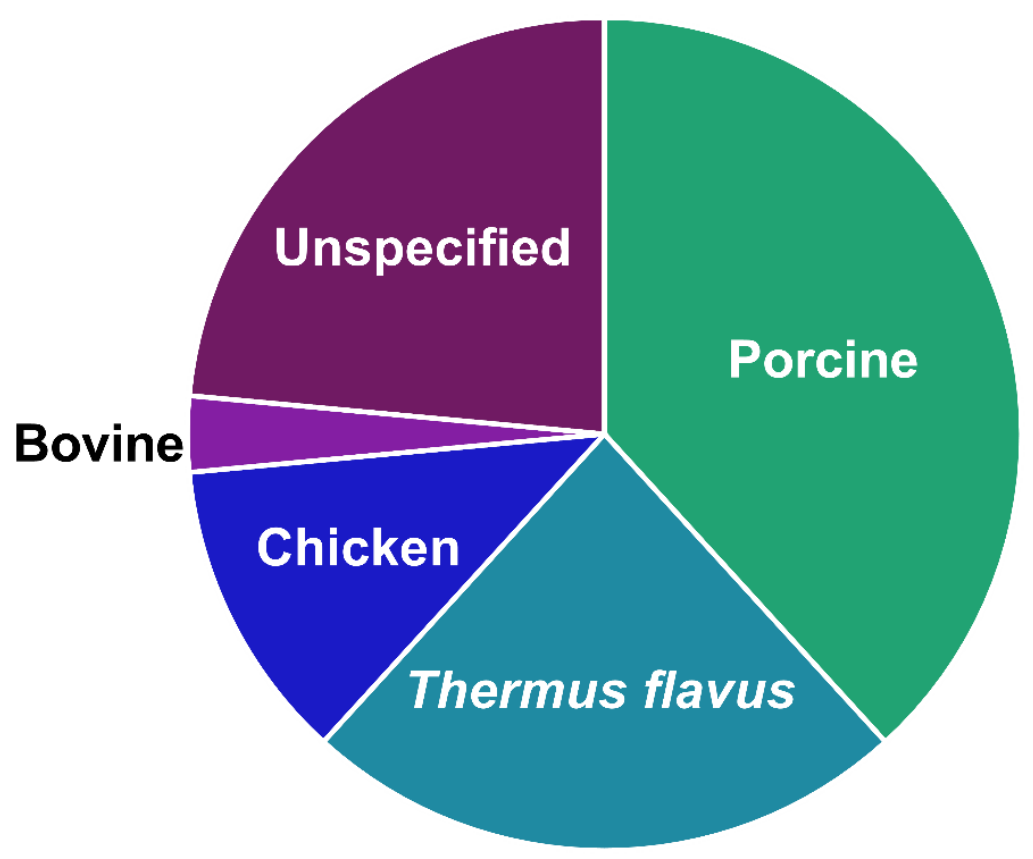

Figure 1.3. The relative number of publications that source the malate oxidoreductase enzyme used the biosensor from each organism. Each type of enzyme shown here can be found as a commercial preparation.

The narrow phylogenetic sampling of malate oxidoreductases (three animals and one thermophilic bacterium) has undoubtedly imparted constraints on the resulting sensors with respect to $\mathrm{pH}$ and temperature optima, enzyme kinetic parameters and storage stability. Environmental conditions that are favourable for any given enzyme can be detrimental to other components of the malate sensor (Gurban et al., 2006) so it seems highly likely that a broader search for new MDHs, MEs and MQOs will prove fruitful. Indeed, at the time of writing (September 2021), the BRENDA enzyme database (https://www.brendaenzymes.org/) (Chang et al., 2020) contains hundreds of entries on functionally and phylogenetically diverse malate oxidoreductases. In the present era of commercial gene synthesis and powerful technologies for protein expression in heterologous hosts, it is now plausible to screen bespoke enzymes from any organism, which may integrate well within a biosensor architecture and simultaneously improve the performance. 


\subsection{Summary and aims}

It has now been 40 years since the first enzyme-based amperometric biosensor for malic acid was described (Blaedel and Engstrom, 1980). In the ensuing decades, key operational parameters including sensitivity, linear range and stability have been improved through the use of coupled enzymes and advances in electrode design. At the same time, the field has remained heavily dependent on a small number of malate oxidoreductase enzymes as the core sensor components. In particular, most biosensors have employed MDHs, whereas promising alternatives such as MEs and MQOs have remained relatively unexplored. Now, in an era of proliferation of sequence databases, coupled with the ease of gene synthesis, it is the right time to investigate if using new types of malate oxidoreductase in can improve the operational parameters of sensors, much in the same way as advances in the non-enzymatic components has achieved.

\section{Aims of this study:}

The work in this thesis seeks to demonstrate that carefully selecting the enzyme used in a biosensor can yield desirable or improved performance characteristics. I focus on solving a particularly prevalent problem in the literature, which is the need for excessive dilution of samples taken from the winemaking process. The overarching hypothesis that I set out to test was that selecting an enzyme with desirable Michaelis-Menten kinetics for malate can be used to produce a sensor with a broad linear range.

The project has three specific aims, addressed in chapters 2, 3 and 4 of the thesis:

1. To expand the repertoire of enzymes used in malate biosensors, by characterising new candidates for their suitability. The literature was searched for enzyme candidates with high reported values for the Michaelis-Menten parameter $K_{\mathrm{M}}$. Candidates were then expressed, purified, and screened for their suitability.

2. To create a working biosensor using the lead candidate chosen from aim 1 . The sensor architecture needed to include an electrode and an electron mediator, both of which had to be compatible with the conditions needed for enzyme activity. 
3. To test the ability of the constructed biosensor to measure malic acid in real samples. As the constituents of grape juice and wine vary depending on the grape cultivar used and the stage of the winemaking process, among other factors, it was important to apply a diverse set of real samples to the device to test for interferences. 


\section{Chapter Two - Screening candidate malate oxidoreductases}

\subsection{Chapter introduction}

The narrow linear ranges of published malate biosensors create the need to use highly dilute samples from the winemaking process. This is unpractical for winemakers, who may be unfamiliar with performing a careful and accurate dilution series. None of the studies reviewed in chapter 1 have explicitly set out to increase the linear range, though an as-yet unexplored strategy is to use enzymes with desirable Michaelis-Menten properties. The principle is outlined in Figure 2.1.

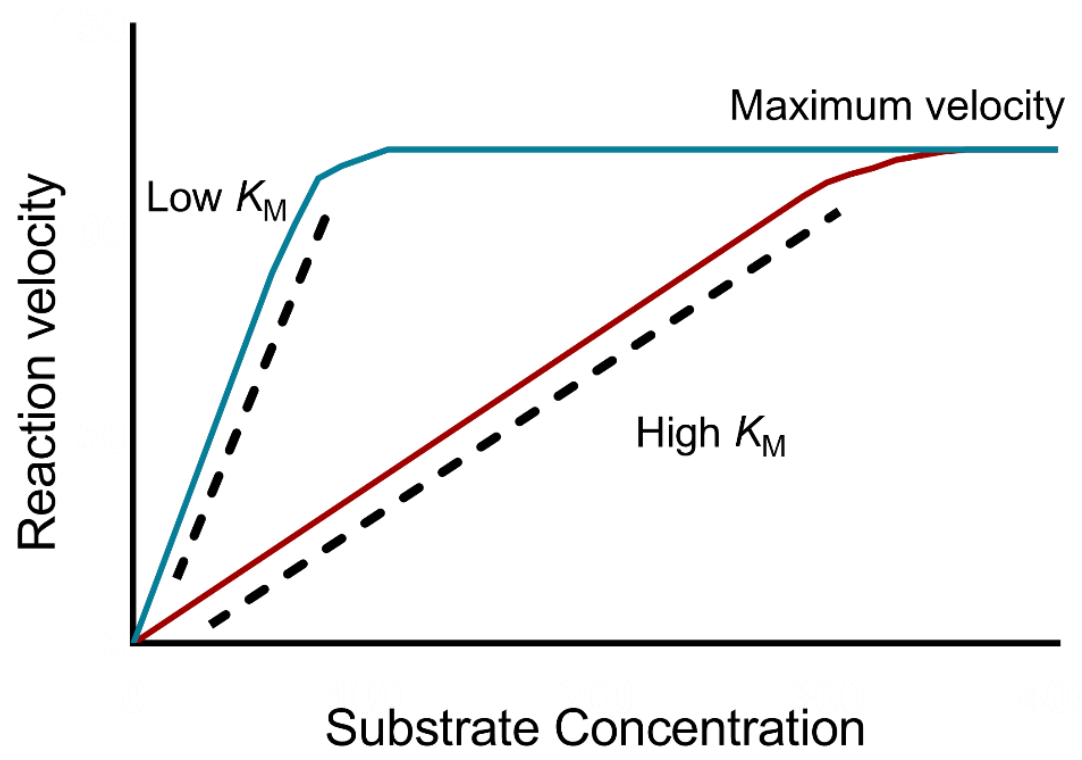

Figure 2.1. Enzyme behaviour as a determinant of a biosensor's linear range. In principle, the linear range of a biosensor (black dashed lines) can be extended by using an enzyme with a greater $K_{\mathrm{M}}$. In the hypothetical example shown here, two malate oxidoreductase enzymes converge on the same maximum reaction velocity at saturating concentrations of their substrate, malate. However, the enzyme with the higher $K_{M}$ (red line) reaches half of this maximum velocity at a higher malate concentration, effectively extending the linear range compared to an enzyme with a lower $K_{\mathrm{M}}$ (blue line). 
The linear range of biosensors is thought to be closely linked to the catalytic behaviour of the enzyme. For enzymes that follow Michaelis-Menten kinetics, the Michaelis constant, $K_{\mathrm{M}}$, is a crucial parameter governing the hyperbolic relationship between increases in analyte concentration and sensor saturation (Silverstein and Goodney, 2010, Thévenot et al., 2001, Vasylieva and Marinesco, 2013). Equation (3) shows the Michaelis-Menten equation, linking the reaction velocity, $V$, to the maximum velocity $\left(V_{\max }\right), K_{\mathrm{M}}$ and the substrate concentration [S]. This has been modified (Vasylieva and Marinesco, 2013) to describe the relationship between increases in analyte concentration and sensor saturation (equation 4), where $K_{\mathrm{M}}{ }^{\mathrm{App}}$ is the apparent $K_{\mathrm{M}}$ of the immobilised enzyme, $I_{\max }$ is the maximum current for the biosensor response and $[\mathrm{S}]$ is the concentration of the enzyme's electroactive substrate (malate, in this case).

$$
\begin{aligned}
& V=\frac{V_{\max }[\mathrm{S}]}{K_{\mathrm{M}}+[\mathrm{S}]} \\
& I=\frac{I_{\max }[\mathrm{S}]}{K_{\mathrm{M}}^{\mathrm{App}}+[\mathrm{S}]}
\end{aligned}
$$

However, it remains unclear to what extent an enzyme's reported $K_{\mathrm{M}}$ can be used to predict the linear range of a device. Table 2.1 shows the reported $K_{\mathrm{M}}$ values of the various commercial preparations used in published malate biosensors. While these values correlate well to the linear ranges of certain published devices (e.g. (Blaedel and Engstrom, 1980), which uses porcine $\mathrm{MDH}$, and has a linear range of $0.006-0.4 \mathrm{mM}$ ), others have linear ranges far narrower or broader than one might expect (Tables 1.1-1.3). For example, the porcine MDH-based sensor by Cho et al. (1995) has a linear range of $0.5-10 \mathrm{mM}$, the upper limit far exceeding the reported $K_{\mathrm{M}}$. 
Table 2.1. The reported $K_{\mathrm{M}}$ values of commercial preparation of malate dehydrogenase $(\mathrm{MDH})$ and malic enzyme (ME).

\begin{tabular}{ccl}
$\begin{array}{c}\text { Commercial } \\
\text { preparation }\end{array}$ & Reported $\boldsymbol{K}_{\mathbf{M}}$ & Reference \\
\hline $\begin{array}{c}\text { Porcine Heart MDH } \\
\text { Bovine Heart MDH }\end{array}$ & $0.4 \mathrm{mM}$ & (Bergmeyer, 1975) \\
Thermus flavus MDH & $0.99 \mathrm{mM}$ & (Grimm and Doherty, 1961) \\
Chicken Liver ME & $11 \mu \mathrm{M}$ & (Nishiyama et al., 1986) \\
\hline
\end{tabular}

For amperometric biosensors, it has been shown that the $K_{\mathrm{M}}{ }^{\mathrm{App}}$ is frequently higher or lower than the $K_{M}$ of the free enzyme (Vasylieva and Marinesco, 2013). Various aspects of the sensor architecture influence enzyme activity, including the immobilisation matrix (Datta $e t$ $a l ., 2013$ ) and co-factor recycling mechanisms that shift of equilibrium of the catalysed reaction (Mizutani et al., 1991). For sensors that use coupled enzyme reactions, the $K_{\mathrm{M}}{ }^{\mathrm{App}}$ may be affected by one enzyme limiting the rate (Bisswanger, 2014).

I hypothesise that linear range remains fundamentally a product of the enzyme's kinetic behaviour, regardless of the architecture used. The goal of this thesis was to extend the linear range by using a malate oxidoreductase with a high $K_{\mathrm{M}}$, and it was simply a matter of using a sensor design that displayed this desired kinetic activity. This chapter describes the screening procedure used to select a lead candidate malate oxidoreductase. High $K_{\mathrm{M}}$ variants, as well as those that potentially afforded extra utility, were identified from the literature, produced as pure protein samples, and had their published characteristics tested empirically. 


\subsection{Results}

\subsubsection{Searching the BRENDA database for candidate malate oxidoreductases}

BRENDA (the Braunschweig Enzyme Database) was used in this study to identify candidate malate oxidoreductases. Widely considered to be the world's most comprehensive source of information on enzymes, the database contains approximately 90,000 entries from 13,000 different organisms. Enzyme parameters, including functional parameters, molecular properties and structural information, have been manually extracted from approximately 157,000 publications in the scientific literature (Chang et al., 2020).

An initial search for enzymes that used L-malate (or the synonymous ' $(S)$-malate') returned 29 distinct enzyme classes. Of these, 14 were oxidoreductases (Enzyme Commission class 1), with seven describing malate as the main substrate. These are shown in Table 2.2. The remaining oxidoreductase classes were given due consideration, though were ultimately excluded for various reasons, including a lack of reported $K_{\mathrm{M}}$ values for malate, catalysed reactions that were not useful for an amperometric sensor, and reaction schemes using malate that were not clear. 
Table 2.2. Seven classes of malate oxidoreductase. The EC (Enzyme Commission) classes that represent my initial search space for candidate malate oxidoreductases. Classes are ordered by ascending EC number, and are grouped by colour corresponding to the enzyme type: Green = malate dehydrogenase, blue $=$ malic enzyme, yellow = malate: quinone oxidoreductase. $\mathrm{NAD}^{+}$or $\mathrm{NADP}^{+}$cofactor preference is indicated by the name of each enzyme.

\begin{tabular}{|c|c|c|}
\hline EC Number & Name & Reaction Catalysed \\
\hline 1.1.1.37 & $\mathrm{NAD}^{+}$malate dehydrogenase & L-malate $+\mathrm{NAD}^{+} \rightleftharpoons$ oxaloacetate $+\mathrm{NADH}$ \\
\hline 1.1.1.38 & $\mathrm{NAD}(\mathrm{P})^{+}$malic enzyme & L-malate $+\mathrm{NAD}(\mathrm{P})^{+} \rightleftharpoons$ pyruvate $+\mathrm{CO}_{2}+\mathrm{NAD}(\mathrm{P}) \mathrm{H}$ \\
\hline 1.1.1.39 & $\mathrm{NAD}^{+}$malic enzyme & L-malate $+\mathrm{NAD}^{+} \rightleftharpoons$ pyruvate $+\mathrm{CO}_{2}+\mathrm{NADH}$ \\
\hline 1.1.1.40 & $\mathrm{NADP}^{+}$malic enzyme & L-malate $+\mathrm{NADP}+\rightleftharpoons$ pyruvate $+\mathrm{CO}_{2}+\mathrm{NADPH}$ \\
\hline 1.1.1.82 & $\mathrm{NADP}^{+}$malate dehydrogenase & L-malate $+\mathrm{NADP}^{+} \rightleftharpoons$ oxaloacetate $+\mathrm{NADPH}$ \\
\hline 1.1.1.299 & $\mathrm{NAD}(\mathrm{P})^{+}$malate dehydrogenase & L-malate $+\mathrm{NAD}(\mathrm{P})^{+} \rightleftharpoons$ oxaloacetate $+\mathrm{NAD}(\mathrm{P}) \mathrm{H}$ \\
\hline 1.1.5.4 & Malate dehydrogenase (quinone) & L-malate + quinone $\rightleftharpoons$ oxaloacetate $+\mathrm{NADH}$ \\
\hline
\end{tabular}

The BRENDA output for these seven enzyme classes cumulatively represents a total of 348 $K_{\mathrm{M}}$ values. The distribution of these $K_{\mathrm{M}}$ values is shown in Figure 2.2. The initial focus was solely on enzymes with the greatest $K_{\mathrm{M}}$ - those with values $>20 \mathrm{mM}$, corresponding to 10 BRENDA entries. Two NAD ${ }^{+}$- dependent malic enzymes were selected from this subset: one from Azorhizobium caulinodans and the other a mutant of the malic enzyme from Ascaris suum. The latter has the highest reported $K_{\mathrm{M}}$ of all malate oxidoreductases in BRENDA, at 57 $\mathrm{mM}$. 


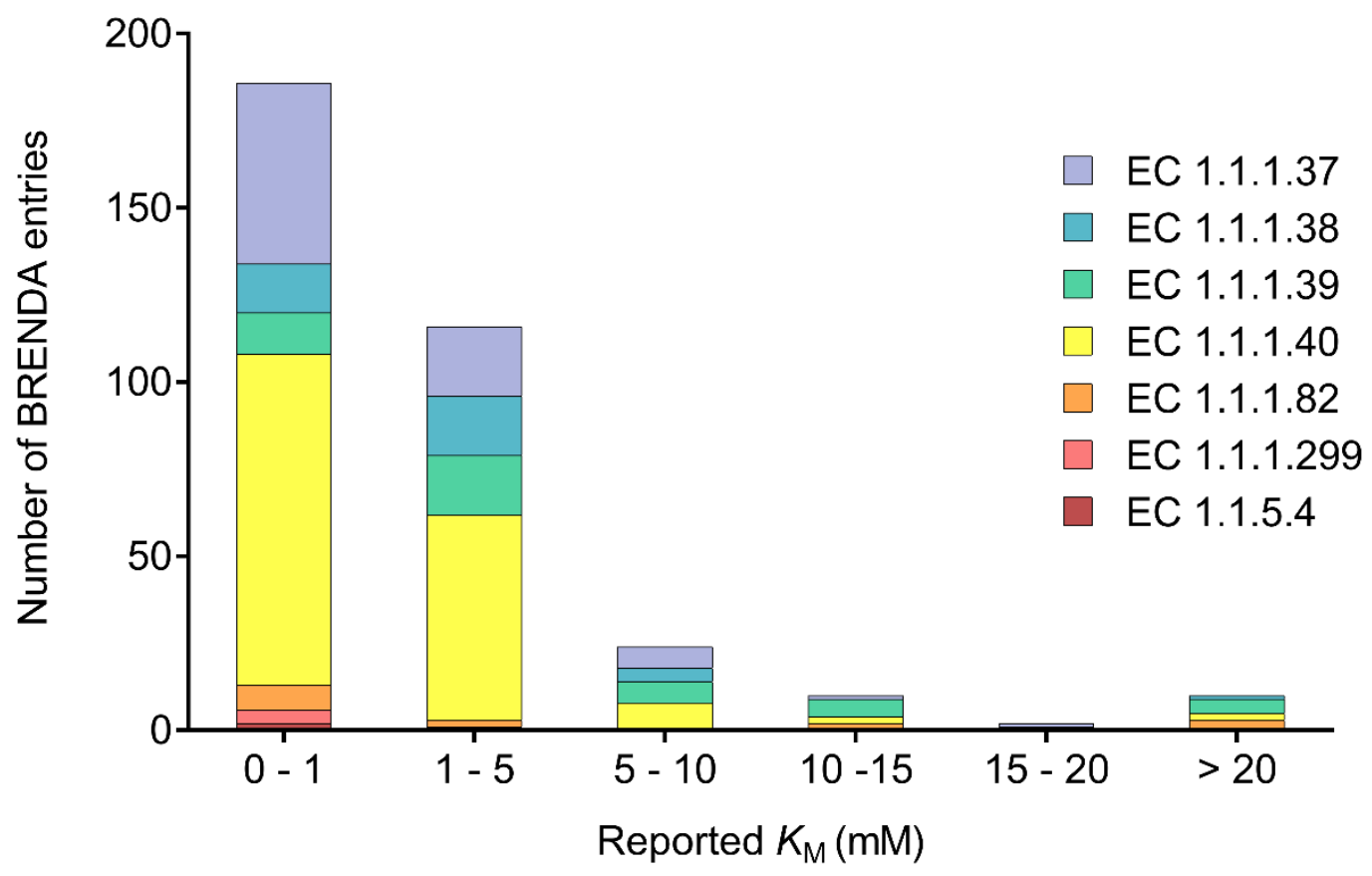

Figure 2.2. Reported $K_{\mathrm{M}}$ values of malate oxidoreductases in the BRENDA database, grouped by their size. Each coloured segment represents a different Enzyme Commission (EC) class.

During the selection of the first two candidates, a secondary selection procedure was applied to screen. This focused on choosing enzymes with characteristics that have the potential to offer novel utility in a biosensor. To be able to consider a wide range of enzyme characteristics, the search was broadened to include enzymes with $K_{\mathrm{M}}$ values less than 20 $\mathrm{mM}$, and led to the selection of 2 more candidates: the $\mathrm{NAD}(\mathrm{P})^{+}-$dependent malate dehydrogenase from Aeropyrum pernix and the $\mathrm{NADP}^{+}$- dependent malate dehydrogenase from Sorghum vulgare.

\subsubsection{Candidate enzyme profiles}

The characteristics, described in the published literature, of each of the four chosen enzymes are described below. For each enzyme, available crystal structures were analysed for the locations of the $\mathrm{N}$-terminal and $\mathrm{C}$-terminal domain. This was used to decide on which end the His $_{6}$-tag, used for purifying proteins, would be attached (described further in section 2.2.3). Three additional malate oxidoreductases, available in our lab, were added to the pool of candidates, and are also described below. 


\subsubsection{AcME - Azorhizobium caulinodans, $\mathrm{NAD}^{+}$- dependent malic enzyme}

Azorhizobium caulinodans is a gram-negative bacterium found within the soil, its genome encodes an $\mathrm{NAD}^{+}$-dependent malic enzyme (AcME; EC 1.1.1.39) which was characterised in a study of the pathways involved in nitrogen fixation (Zhang et al., 2012). The predicted structure is shown in Figure 2.3. Various compounds have been shown to modulate the $K_{\mathrm{M}}$ for malate. Of particular interest to this study, the addition of $50 \mu \mathrm{M}$ acetyl-CoA to in vitro assays increased the $K_{\mathrm{M}}$ from $2.6 \mathrm{mM}$ to $27.6 \mathrm{mM}$, an approximate 10-fold increase (Zhang et al., 2012). An increase of this co-factor to $100 \mu \mathrm{M}$ appears to increase the $K_{\mathrm{M}}$ further still, as shown by activity versus malate concentration plots, though the value is not reported.

Succinate and fumarate also modulated the Michaelis-Menten kinetics, though they both caused an approximate 4-fold decrease in $K_{\mathrm{M}}$ when used at concentrations of $10 \mathrm{mM}$ and 1 $\mathrm{mM}$ respectively (Zhang et al., 2012). I hypothesised that the ability to modify the kinetics parameters with small molecule additives might be useful for optimizing a biosensor.
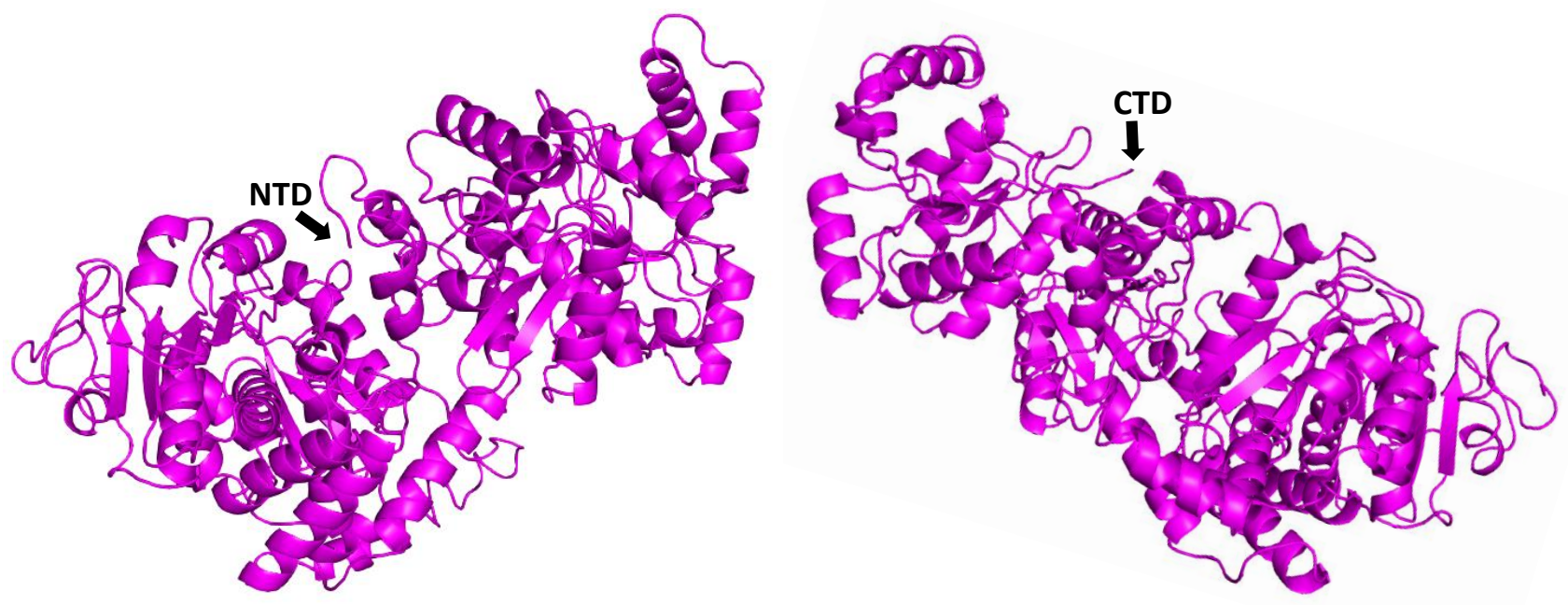

Figure 2.3. PHYRE2 homology model of the $\mathrm{NAD}^{+}$-dependent malic enzyme from Azorhizobium caulinodans. No crystal structure for the enzyme exists. The predicted structure is shown in two different orientations to clearly show the locations of the N-terminal (NTD) and C-terminal (CTD) domains. My analysis determined that neither domain is sequestered by the by the bulk of the structure. The figure was created using PyMOL. 


\subsubsection{AsME - Ascaris suum, NAD+- dependent malic enzyme}

Multiple studies have characterised the $\mathrm{NAD}^{+}$- dependent malic enzyme from Ascaris suum (AsME, EC 1.1.1.39) (Coleman et al., 2002, Rao et al., 2003, Karsten et al., 2003). However, the choice to select this enzyme as a candidate was based on the findings of Karsten and Cook (2007). The study investigated the role of arginine 181 (R181) in binding malate within the active site. Substituting R181 with glutamine yielded a mutant with a $K_{\mathrm{M}}$ for malate of 57 $\mathrm{mM}$, approximately 100-fold greater than that of the wild-type. The high $K_{\mathrm{M}}$ of the mutant was attributed to the loss of the guanidinium group present in arginine, which acted to disrupt the co-ordination of malate. This is visualised in Figure 2.4.

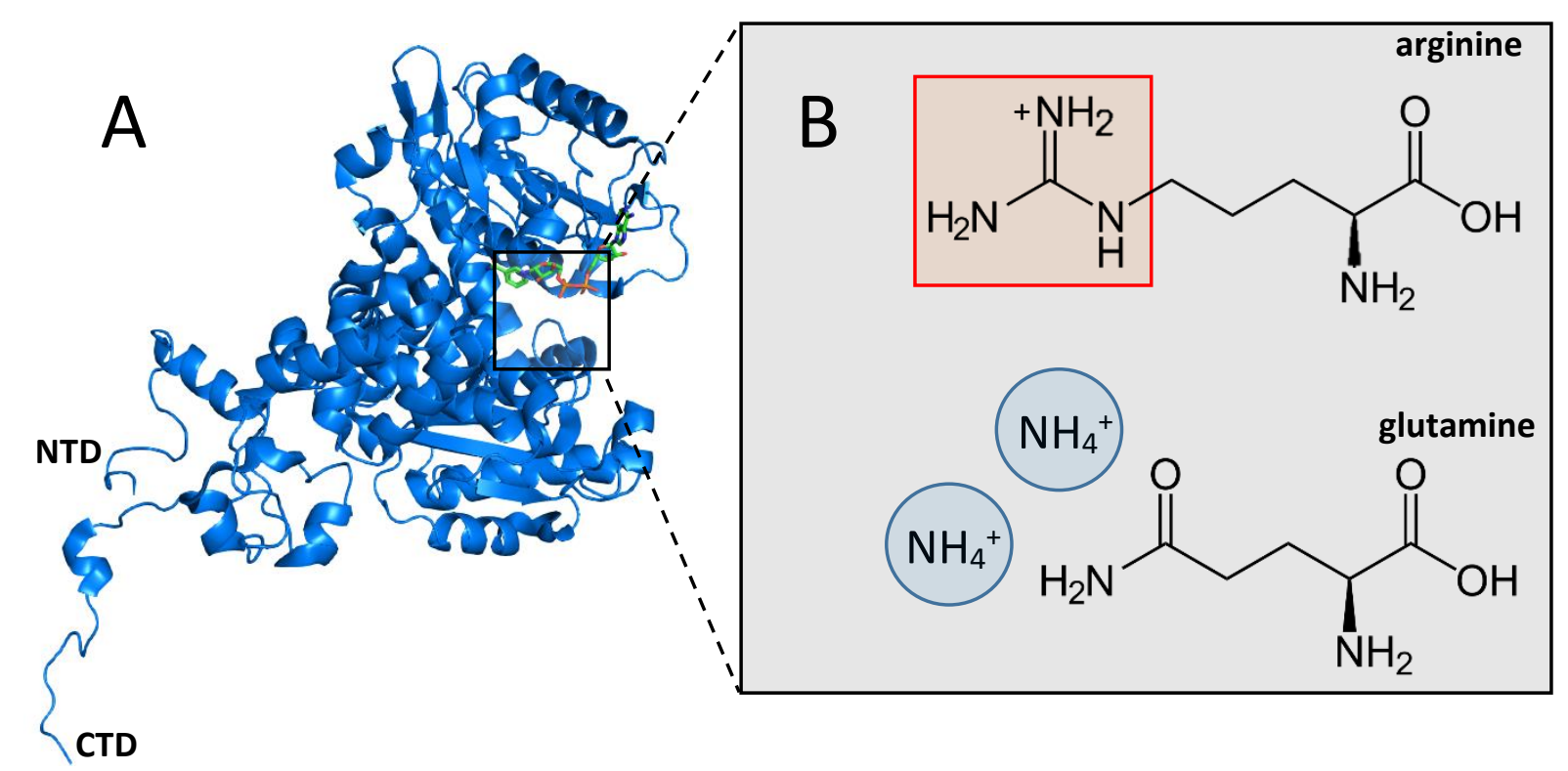

Figure 2.4. A) Ribbon structure of the monomeric subunit of AsME, which crystallises as a tetramer. PDB ID 1LLQ. N-terminal (NTD), C-terminal (CTD) domains and the active site are highlighted, with the precise residues involved in malate binding not being known. B) Visualisation of the R181Q mutation and the proposed recovery mechanism. Substituting arginine for glutamine resulted in an increased $K_{M}$ for malate, and a decreased $k_{\text {cat, }}$ compared to the wild-type. This behaviour has been attributed to the loss of the guanidinium group, which has been proposed to be involved in coordinating malate within the active site. The MichaelisMenten kinetics of the mutant can be modified by the addition of ammonium to the assay mixtures, thought to be due to two molecules of ammonia acting in place of the two 'lost' nitrogen groups of the guanidinium moiety. 
This increase in $K_{\mathrm{M}}$ is accompanied by an approximate 17 -fold decrease in $k_{\text {cat }}$. However, the authors describe a method of 'chemical rescue'. Adding ammonium $\left(\mathrm{NH}_{4}^{+}\right)$, up to concentrations of $25 \mathrm{mM}$ can be used to decrease the $K_{\mathrm{M}}$, while $k_{\text {cat }}$ remains relatively constant. However, at higher concentrations, > $25 \mathrm{mM}$, increases in $k_{\text {cat }}$ are observed instead, while the $K_{\mathrm{M}}$ remains relatively unchanged. This trend is shown in Table 2.3. A mechanism is proposed for this behaviour, where two $\mathrm{NH}_{4}{ }^{+}$molecules act in place of the two nitrogen atoms of the guanidinium group. The decrease in $K_{\mathrm{M}}$ is thought to be due to binding of $\mathrm{NH}_{4}{ }^{+}$ to a 'high-affinity' site, with a second, 'low-affinity' site being occupied at higher concentrations, resulting in the increase of $k_{\text {cat }}$ (Karsten and Cook, 2007). Like for AcME, being able to modify the Michaelis-Menten kinetics using chemical additives may be useful for tuning the parameters of a biosensor.

Table 2.3. Changes in the Michaelis-Menten parameters of AsME in response to ammonia. Values are those reported by Karsten and Cook (2007). Orange and blue wedges indicate the trends in $K_{\mathrm{M}}$ and $k_{\text {cat }}$ as ammonium concentration is increased. $K_{\mathrm{M}}$ values reflect concentrations of free malate - uncomplexed with the $\mathrm{NAD}^{+}$and $\mathrm{Mg}^{2+}$ present within assays.

\begin{tabular}{ccc}
$\mathbf{N H}_{4}{ }^{+}(\mathbf{m M})$ & $\boldsymbol{K}_{\mathbf{M}}$ malate $(\mathbf{m M})$ & $\boldsymbol{k}_{\text {cat }}\left(\mathbf{s}^{-\mathbf{1}}\right)$ \\
\hline 0 & 57 & 1.6 \\
1.5 & 21 & 1.5 \\
4 & 12 & 1.7 \\
6 & 8 & 1.7 \\
10 & 8 & 1.9 \\
25 & 6 & 3.3 \\
60 & 8 & 5.8 \\
120 & 8 & 10.5 \\
300 & 13 & 19 \\
600 & 10 & 34 \\
\hline
\end{tabular}

\subsubsection{SvMDH - Sorghum vulgare, $\mathrm{NADP}^{+}$- dependent malate dehydrogenase}

Of the eukaryotic malate dehydrogenase isoforms, cytosolic and mitochondrial are most often described. However, a third exists, an $\mathrm{NADP}^{+}$-dependent $\mathrm{MDH}$ found within the chloroplasts of higher plants. This enzyme offers a mode of regulation unique among the isoforms - light activation. The $\mathrm{NADP}^{+}$- dependent malate dehydrogenase from the chloroplast of Sorghum 
vulgare (SMDH, EC 1.1.1.82) is inactive in the dark. However, upon absorption of light, a relay of redox reactions, the ferredoxin-thioredoxin system, results in the reduction of two regulatory disulfide bridges, activating the enzyme. In vitro, this activation can be induced using a simplified system, using thioredoxin and the non-physiological reducing agent dithiothreitol. Conversely, oxidation by glutathione results in deactivation (Wolosiuk et al., 1977). It was thought that these mechanisms could be exploited to afford greater control over a biosensor through a chemically induced on/off switch. Moreover, at $12 \mathrm{mM}$, the $K_{\mathrm{M}}$ appears to be inherently high - no mutations or special additives are required to observe this value, minimising the number of reagents required for the sensor assay (Lemaire et al., 1996). The predicted structure of SvMDH is shown in figure 2.5.

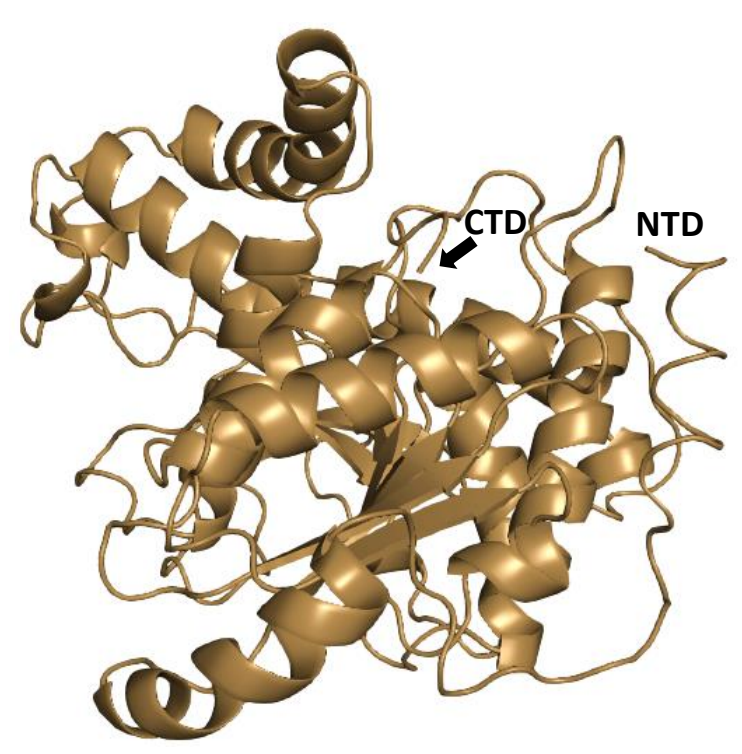

Figure 2.5. PHYRE2 homology model of the $\mathrm{NADP}^{+}$-dependent malate dehydrogenase from Sorghum vulgare. No crystal structure for the enzyme has been published. The predicted locations of the N-terminal (NTD) and C-terminal (CTD) domains are shown, and it was determined that neither is sequestered by the bulk structure. The figure was created using PyMOL.

\subsubsection{ApMDH - Aeropyrum pernix, $\mathrm{NAD}(\mathrm{P})^{+}$- dependent malate dehydrogenase}

The hyperthermophilic archaeon Aeropyrum pernix produces a $\mathrm{NAD}(\mathrm{P})^{+}$- dependent malate dehydrogenase (ApMDH, EC 1.1.1.299). This malate oxidoreductase does not have a high $K_{\mathrm{M}}$ for malate like other candidates, at $0.12 \mathrm{mM}$ when using $\mathrm{NAD}^{+}$as the cofactor, and 0.019 $\mathrm{mM}$ when using $\mathrm{NADP}^{+}$(Kawakami et al., 2009). However, the enzyme was chosen for the 
described high $\mathrm{pH}$ stability over a wide range of $\mathrm{pH}$ values, and to a lesser extent, its high thermostability. I hypothesised that ApMDH had the potential to create a robust biosensor that remains stable when subjected to mildly acidic grape juice.

The primary literature by Kawakami et al. (2009) states: "when heated at $50{ }^{\circ} \mathrm{C}$ for 30 minutes the enzyme showed no loss of activity at pHs between 5.5 and 10.5 ". This description was interpreted as the activity of ApMDH being unaffected despite large differences in the $\mathrm{pH}$ used for assays, including a shift from alkaline to mildly acidic conditions. The high thermostability is shown through the retention of $100 \%$ activity even after being heated at $100^{\circ} \mathrm{C}$ for 10 minutes, with activity during assays increasing with temperature up to at least $95^{\circ} \mathrm{C}$. The crystal structure of ApMDH is shown in figure 2.6.
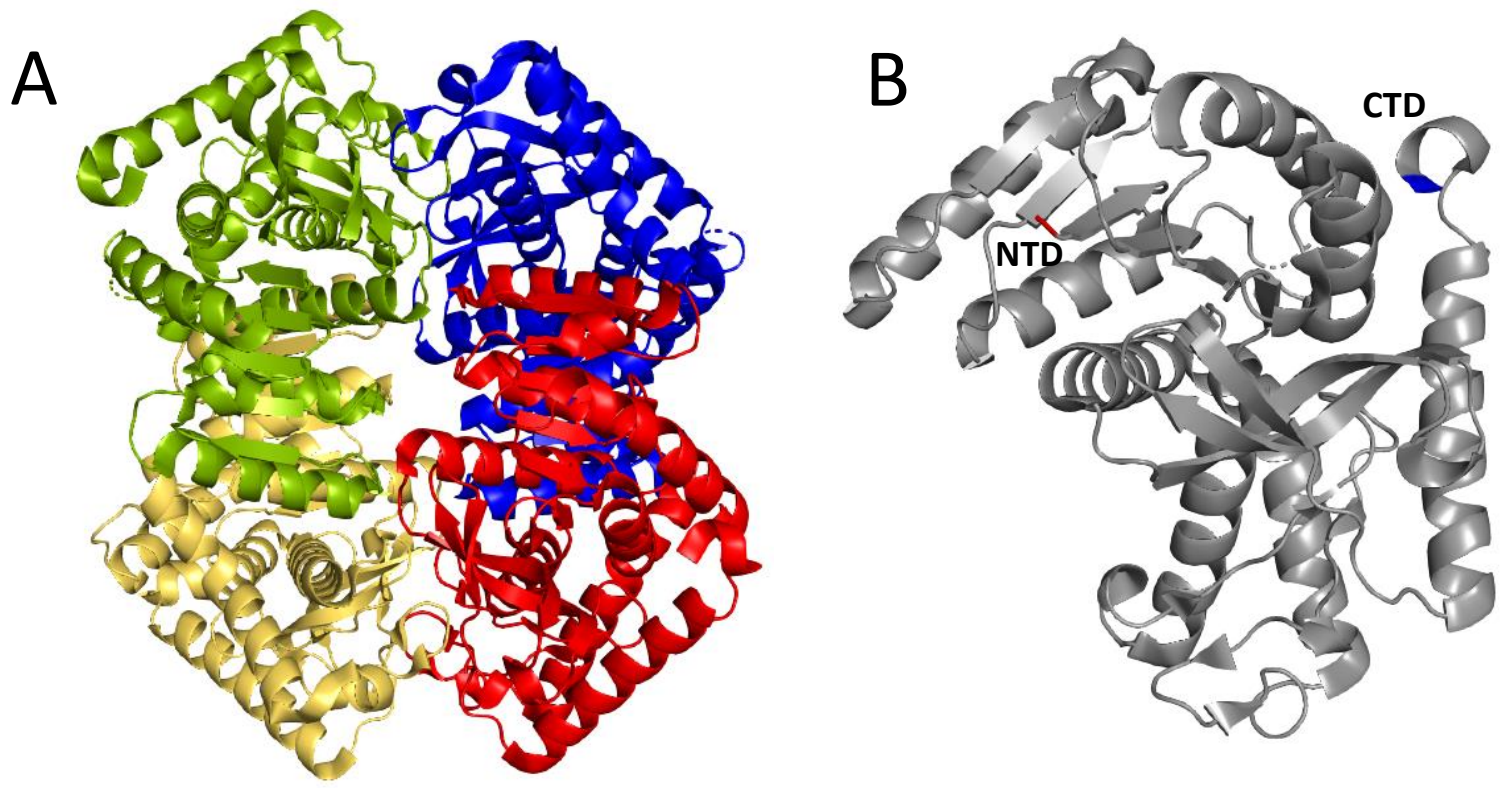

Figure 2.6. Crystal structure of the $\mathrm{NAD}(\mathrm{P})^{+}$- dependent malate dehydrogenase from Aeropyrum pernix. A) The enzyme crystallises as a tetramer, with each subunit being shown as a different colour. B) The monomer subunit. The N-terminal (NTD) and C-terminal (CTD) domains highlighted in red and blue, respectively. The Nterminal domain is located within the $\beta$-sheet of a Rossman fold, surrounded by $\alpha$-helices on either face that may sequester an attached His6-tag. The C-terminal domain is more solvent exposed due to its location at the end of an $\alpha$-helix that forms the outer structure of the enzyme. 


\subsubsection{TmME - Thermotoga maritima, putative malic enzyme}

The putative malic enzyme from the Thermotoga maitima has no information published on its functional parameters, though a tetrameric crystal structure exists under the PDB accession code 1VL6. As T. maritima is thermophilic, found inhabiting hot springs and hydrothermal vents with water temperatures up to $90^{\circ} \mathrm{C}$ (Huber et al., 1986), it is likely that TmME too, is thermophilic. As described in section 1.9, thermostable enzymes can be used to create extraordinarily robust devices, with long storage stabilities. The presence of a solved structure would also make a potential enzyme-engineering endeavour easier if required.

\subsubsection{MaeA - Escherichia coli $\mathrm{K} 12, \mathrm{NAD}(\mathrm{P})^{+}$malic enzyme}

The genome of Escherichia coli strain K-12 encodes two malic enzymes, one a NAD $(\mathrm{P})^{+}$ dependent (MaeA, EC 1.1.1.38), the other a NADP ${ }^{+}$- dependent (MaeB, EC 1.1.1.40). MaeA was used in this study. The enzyme has been characterised, in terms of the Michaelis-Menten kinetics and the compounds that regulate its activity, through multiple studies (Bologna et al., 2007, Kwon et al., 2007, Ganesh et al., 2013). Using NAD ${ }^{+}$as co-factor, the $K_{\mathrm{M}}$ reported for malate was $0.66 \mathrm{mM}$, while the use of $\mathrm{NADP}^{+}$produced a sigmoidal curve when increasing malate. It is unclear if such a non-hyperbolic curve can be used in a biosensor. Various compounds affect the activity of MaeA, that is, the change in rate was measured at a set concentration of malate, as opposed to measuring the difference in the Michaelis-Menten kinetics: MaeA is activated by aspartate and inhibited by acetylphosphate, palmitoyl-CoA and oxaloacetic acid (Bologna et al., 2007).

\subsubsection{Cloning, expression and purification of chosen candidates}

The process to obtain pure samples of each candidate started with the design of recombinant vectors that contained the gene of each malate oxidoreductase. To enable purification by immobilised metal affinity chromatography, each expression product was to include a His6tag, fused on to either the $\mathrm{N}$ - or C-terminal end of the amino acid sequence.

There is a possibility for protein purification to fail if the His ${ }_{6}$-tag is sequestered by the bulk of the protein structure. Based on the analyses of the structures (described above), an N- 
terminal His $6^{-}$tag was used for AcME, AsME(R181Q) and SvMDH, whereas a C-terminal tag was used for ApMDH.

The DNA sequences of these four candidates were purchased from a commercial gene synthesis company (Twist Bioscience), cloned into the pET-28a(+) expression vector. The genes for the other three malate oxidoreductases, TmME and the two E.coli enzymes, were available in our laboratory as members of two different open reading frame collections. The gene encoding TmME was available in the arabinose-inducible expression vector pMH1, constructed as part of a structural genomics project (Lesley et al., 2002). The gene encoding MaeA was in the isopropyl $\beta$-D-1-thiogalactopyranoside (IPTG)-inducible expression vector pCA24N (Kitagawa et al., 2005), with the gene for green fluorescent protein (GFP; 242 amino acids) fused to their C-termini. It was unknown whether this C-terminal GFP fusion would hinder the malate oxidoreductase activity, so it was removed. This involved digesting pCA24N-maeA and with NotI to remove the GFP gene, excising the digested vector from an agarose gel, re-circularising it with T4 DNA ligase, and using the ligated product to transform E. coli strain E. cloni $10 \mathrm{G}$ by electroporation. The relevant methods are described in sections 6.2 and 6.3 .

Initial attempts to express the four candidates from BRENDA, AcME, AsME(R181Q), ApMDH and SvMDH, used LB medium, $1 \mathrm{mM}$ IPTG, and an overnight incubation at $25^{\circ} \mathrm{C}$. However, SvMDH, AcMDH, ApMDH expressed as abundant insoluble proteins. AsME(R181Q) was less abundant, though was still insoluble. The first attempt to optimise expression used a reduced inducer concentration, $50 \mu \mathrm{M}$ IPTG, though this did not result in the production of soluble protein either, only a reduced abundance of all candidates.

Changing the growth medium has been reported to improve the solubility of certain proteins (Francis and Page, 2010). The next set of expression trials used autoinduction media instead of LB. Timepoint samples were taken at 1, 3 and 5 hours during the incubation period. Overexpression bands were best seen for the samples taken after 5 hours. While this did not improve the solubility of ApMDH and SvMDH, for both AcME and AsME(R181Q), the expressed protein appeared in both the insoluble and soluble fractions in equal abundance.

To build on these promising results, further optimisation trials also used autoinduction media, but reduced the incubation temperature to $16^{\circ} \mathrm{C}$. A longer incubation time, up to 48 hours, was also tested for each of the four candidates, as well as varying the type of buffer used for cell lysis. These results are shown in Figure 2.7. Soluble AcME and AsME(R181Q) proteins 
were highly abundant in time point samples taken at 12 and 48 hours, though similarly-sized overexpression bands was also seen in the insoluble fraction for AcME. The time point sample taken at 48 hours, when lysed in lysis buffer that contained $100 \mathrm{mM}$ HEPES, produced the greatest amount of soluble protein for AcME, while the type of lysis buffer had no effect on the solubility of AsME(R181Q).

The effect of these conditions on the expression of soluble SvMDH and ApMDH was less clear. For SvMDH, it at first appeared that overexpressed protein was present in the soluble fraction of the 12 hour timepoint. However, upscaled expression using these conditions failed to yield any soluble protein, suggesting that the band seen was an artifact caused by overloaded adjacent lanes. For ApMDH, insoluble protein was abundant in 12- and 48-hour time point samples, though smaller bands of the same molecular weight were seen in the corresponding soluble fractions. Upscaled expression and the subsequent purification by affinity chromatography revealed that these bands were due to small amounts of soluble ApMDH. 

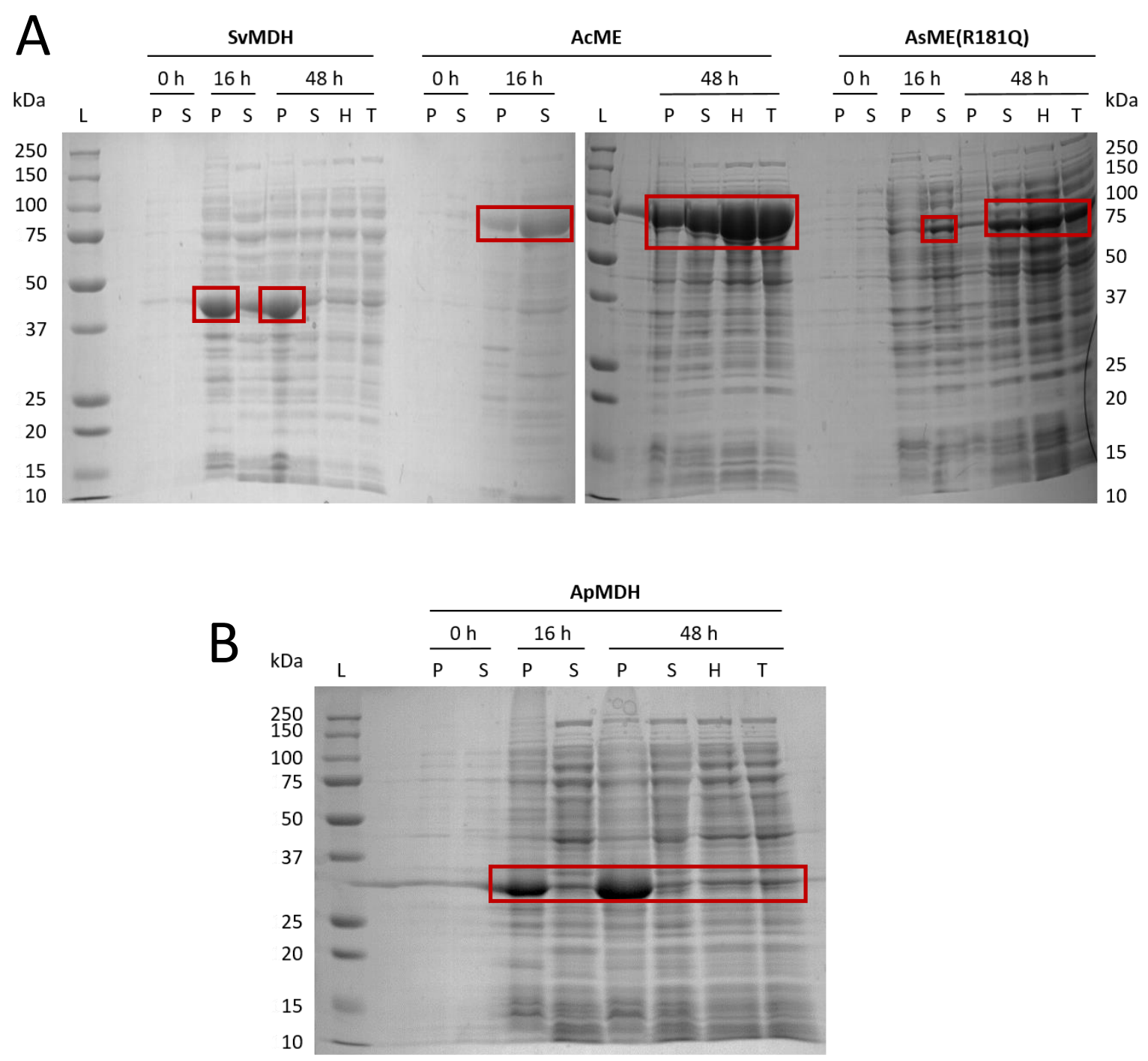

Figure 2.7. SDS-PAGE gels of expression trials of A) SvMDH, AcME and AsME(R181Q), and B) ApMDH. Each protein was expressed using the BL21(DE3) Gold expression strain grown in autoinduction media and an incubation temperature of $16^{\circ} \mathrm{C}$. The incubation time was varied from 0 to $48 \mathrm{~h}$. $\mathrm{L}=$ Precision Plus Protein Standard, $\mathbf{P}=$ "pellet", insoluble fraction of lysed cells, $\mathbf{S}=$ "soluble" fraction of cells lysed in $100 \mathrm{mM}$ potassium phosphate buffer $\mathrm{pH} \mathrm{7,} \mathrm{H} \mathrm{=} \mathrm{soluble} \mathrm{fraction} \mathrm{of} \mathrm{cells} \mathrm{lysed} \mathrm{in} 100 \mathrm{mM}$ HEPES buffer $\mathrm{pH}$ 7, T= soluble fraction of cells lysed in $100 \mathrm{mM}$ Tris- $\mathrm{HCl}$ buffer $\mathrm{pH}$ 7. Additional constituents of each lysis buffer are described in section 6.4.4. Overexpression bands corresponding to the size of the candidate are highlighted in red rectangles.

Only SvMDH remained a challenge to express and purify. The final attempt at obtaining soluble SvMDH involved co-expression with a molecular chaperone. E. coli BL21(DE3) Gold containing pET-28a(+)/SVMDH was subsequently re-transformed to also include the 
pGro7 plasmid - a commercial plasmid able to express the two subunits of the GroES GroEL chaperone complex. Visualisation of the co-expression by SDS-PAGE showed overexpressed bands corresponding to the size of GroEL and GroES in the soluble fraction, though the band corresponding to SvMDH remained entirely within the insoluble fraction. After these multiple attempts of obtaining soluble protein, SvMDH was removed as a candidate for the biosensor.

MaeA and TmME, were successfully expressed under the first conditions that were tried. These conditions, along with those that were successful for other candidates are shown in Table 2.4.

Table 2.4. Summary of the protein expression conditions used to obtain soluble protein samples of each malate oxidoreductase candidate

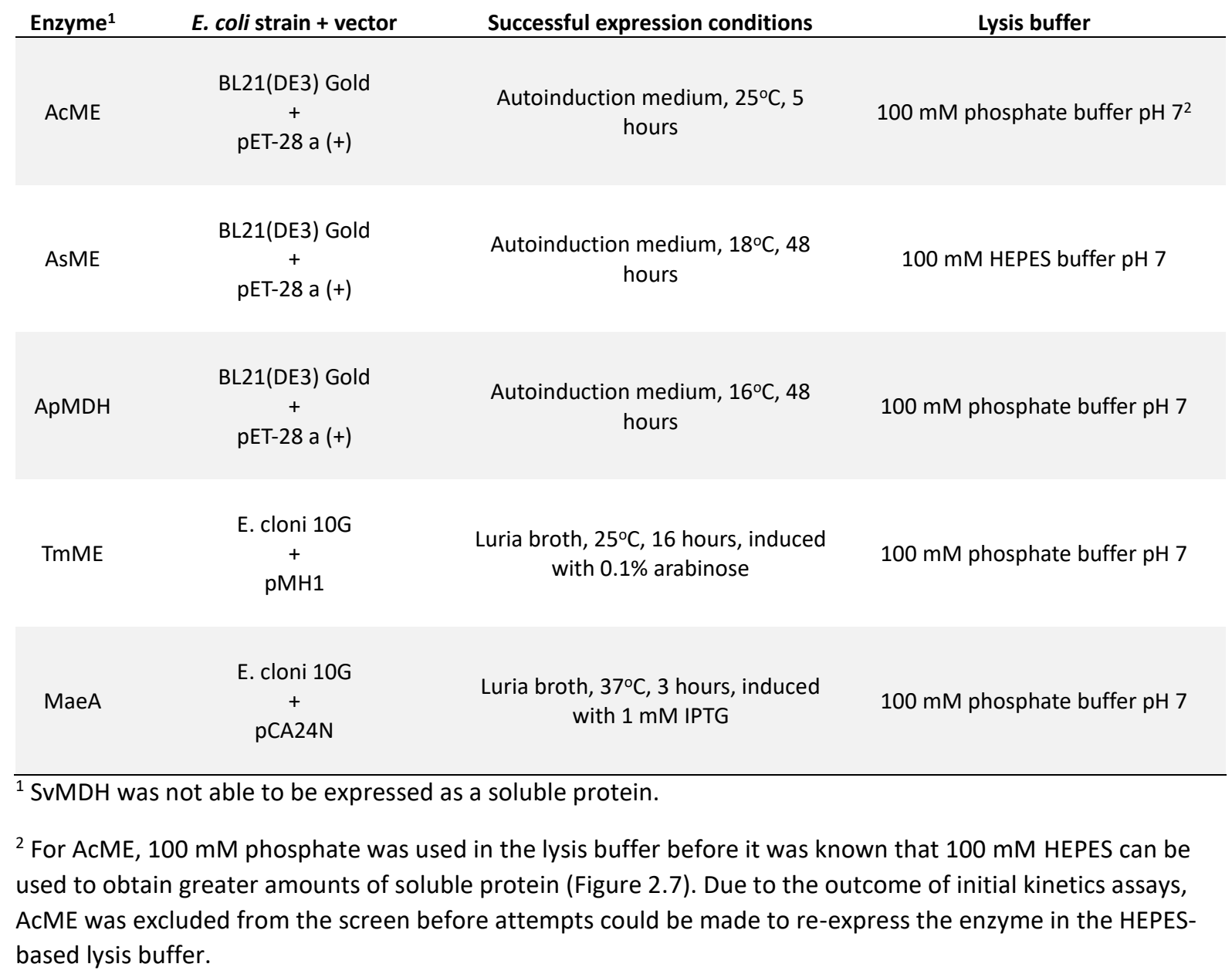


Using the conditions in Table 2.4, each candidate was expressed using upscaled cultures (500 $\mathrm{mL}$ ), then purified using affinity chromatography using Ni-NTA resin and the ÄKTA system (see section 6.4.4.1). The exception was ApMDH, which was purified using TALON resin and gravity flow (section 6.4.4.2). The success of the purification was judged by eye, with samples being deemed sufficiently pure if the desired protein made up at least $90 \%$ of the total protein in each sample. For MaeA and ApMDH, affinity chromatography alone was sufficient to obtain pure samples, though for AsME(R181Q), AcME and TmME further purification by size-exclusion chromatography was necessary. The purified products are shown in Figure 2.8. 
AcME

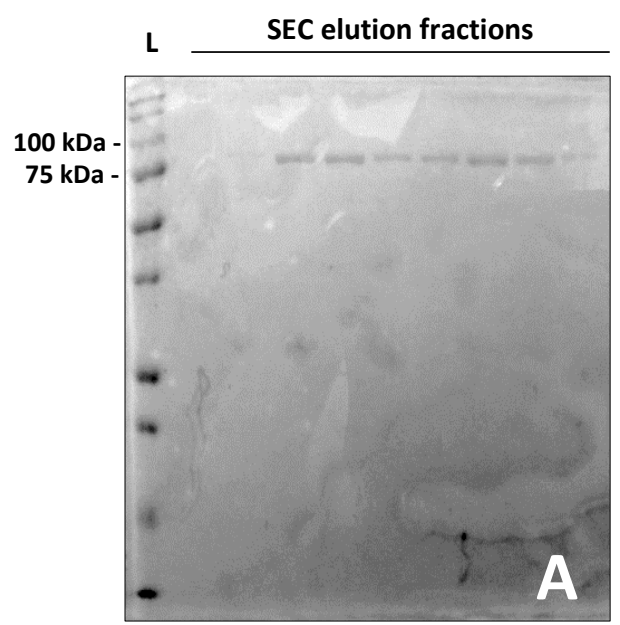

Expected size: 81.8 kDa

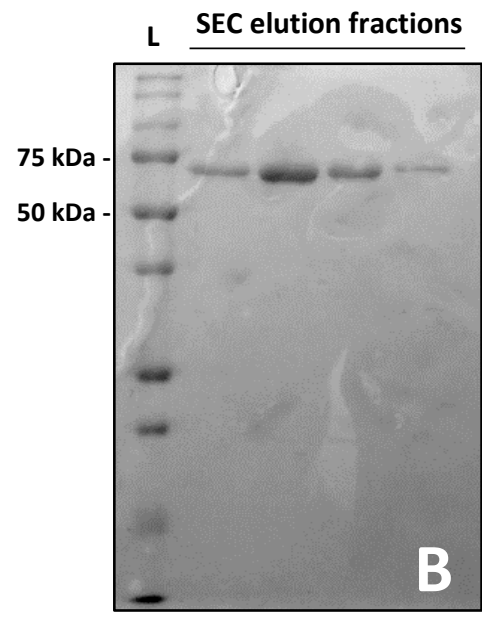

Expected size: $68.6 \mathrm{kDa}$
ApMDH

Elution fractions

L $\quad 500 \mathrm{mM}$ imidazole

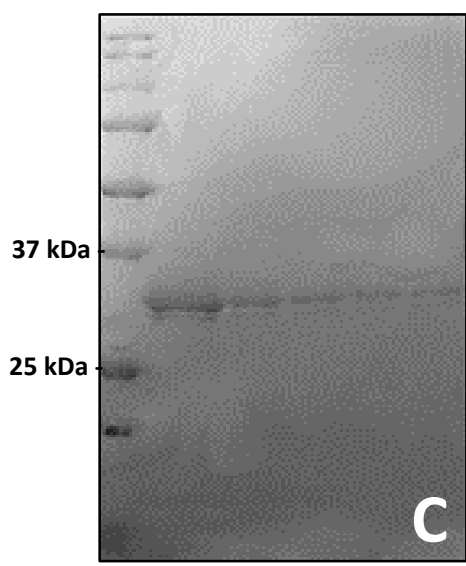

Expected size: 34.7 kDa

\section{TmME}

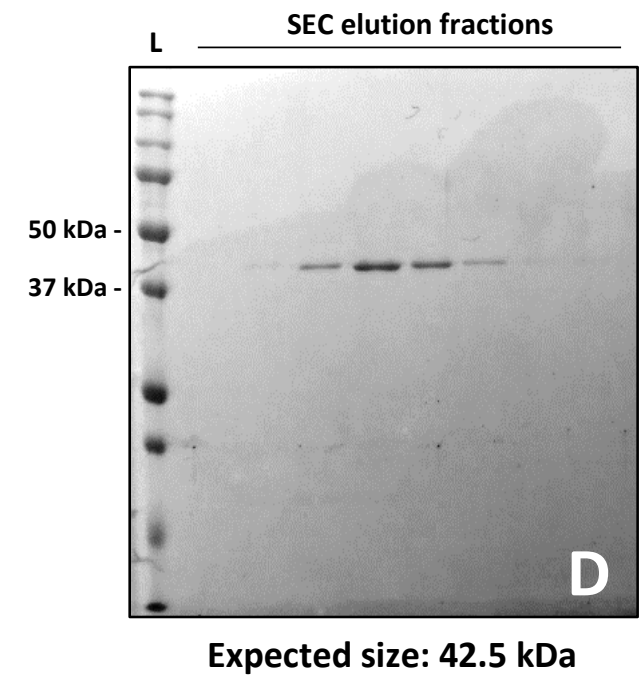

MaeA

Elution fractions

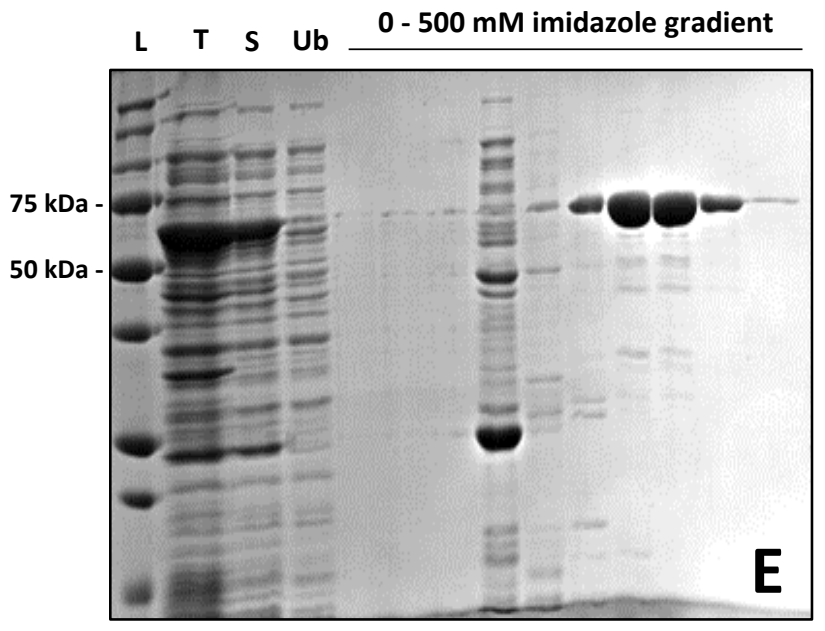

Expected size: $65.5 \mathrm{kDa}$

Figure 2.8. SDS-PAGE gels of solubly expressed and purified candidate malate oxidoreductases. Gels for $\mathbf{A}$ ) AcME, B) AsME(R181Q) and D) TmME shows the protein obtained from the elution fractions from sizeexclusion chromatography (SEC) after previously having been purified by metal affinity chromatography. The corresponding SEC chromatograms are shown in Appendix 1. C) ApMDH and E) MaeA show proteins after metal affinity chromatography alone. $\mathrm{L}=$ Precision Plus Protein prestained ladder, $\mathrm{T}$ = 'total protein', the sample obtained after centrifugation, $\mathrm{S}=$ the "soluble fraction", obtained after centrifugation, Ub = "unbound protein", the flow through after column loading. 


\subsubsection{Kinetic characterisation}

The remaining five candidates were subjected to kinetic characterisation. The aim was to validate the Michaelis-Menten parameters given in the published literature and to identify any undescribed characteristics that may benefit the construction of a biosensor. Aliquots of each purified enzyme were tested for their ability to oxidise malate in vitro, using the appropriate cofactor (either $\mathrm{NAD}^{+}$of $\mathrm{NADP}^{+}$). The protocols used and the specific constituents of assays are described in section 6.5.

\subsubsection{AcME}

The change in the initial velocity $\left(V_{0}\right)$ of $A c M E$ was measured when increasing the malate concentration from $0-100 \mathrm{mM}$, using $1.5 \mathrm{mM} \mathrm{NAD}^{+}$as the co-factor (Figure 2.9). The $K_{\mathrm{M}}$ reported by Zhang et al. (2012) is $27.6 \mathrm{mM}$ when using $50 \mu \mathrm{M}$ acetyl-coenzyme A, with an increase to $100 \mu \mathrm{M}$ appearing to increase the $K_{\mathrm{M}}$ further, though an exact value is not given. My investigation used a different co-factor, coenzyme A (coA), on the hypothesis that its structural similarity to acetyl-CoA would produce similar $K_{\mathrm{M}}$-increasing effects.

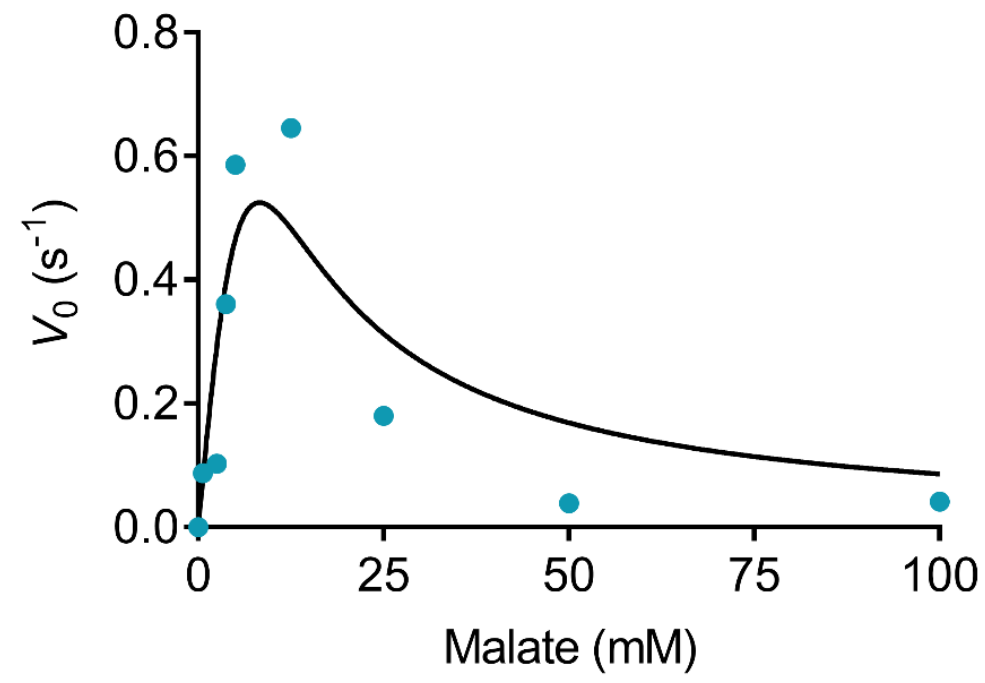

Figure 2.9. The kinetic behaviour of $A c M E$. The initial velocity $\left(V_{0}\right)$ is plotted against increasing concentrations of malate. Assays used $100 \mu \mathrm{M}$ coenzyme A. Points have been fitted with the Haldane model for substrate inhibition. Replicates were not performed due to the instability of the enzyme causing it to be removed from the screen. 
$V_{0}$ increased as malate was increased from $0-12.5 \mathrm{mM}$, and the highest velocity observed was $0.65 \mathrm{~s}^{-1}$. However, at concentrations above $12.5 \mathrm{mM}$, the enzyme activity sharply declined in a hyperbolic fashion towards zero. As this trend was the result of increasing malate concentration, it was thought to be the product of substrate inhibition. However, as shown in Figure 2.9, the model of substrate inhibition fits poorly to the plotted points, which indicates that a non-classical mode of substrate inhibition was influencing the kinetics. Such kinetic behaviour is not seen in the study by (Zhang et al., 2012), where increases in activity were observed up to the maximum concentration tested, $40 \mathrm{mM}$. Despite potentially having use in a biosensor for measuring malate concentrations up to a maximum of $12.5 \mathrm{mM}$, such substrate inhibition was ultimately deemed undesirable for creating the biosensor, as malate concentrations present in the winemaking process often exceed $12.5 \mathrm{mM}$, and the calibration plot allows for two different concentrations of malate give the same readout.

As assays were performed, the aliquots of AcME were stored on ice. After approximately 6 hours, a sharp decline in activity was observed to rates approximately $10 \%$ of the freshly thawed enzyme. Aliquots also formed a white precipitate, indicating that AcME had aggregated. For this instability, and the substrate inhibition described above, AcME was not taken forward for further characterisation.

\subsubsection{TmME}

No Michaelis-Menten kinetics had previously been reported for TmME, so I aimed to characterise the kinetics for the first time. It should be noted that the Michaelis-Menten kinetics described here for TmME are 'apparent', as initial assays, before the optimisation of co-factor concentrations, were sufficient to decide on TmME's suitability in a biosensor.

Kinetics assays were first performed at $50^{\circ} \mathrm{C}$, with $1 \mathrm{mM} \mathrm{NAD}^{+}$and $0.5 \mathrm{mM} \mathrm{Mg}^{2+}$ as cofactors. Increasing malate from $0-10 \mathrm{mM}$ yielded a plot of initial rates that could be fitted to the Michaelis-Menten equation: apparent values for $k_{\text {cat }}$ and $K_{\mathrm{M}}$ were $4.2 \mathrm{~s}^{-1}$ and $1.8 \mathrm{mM}$, respectively (Figure 2.10).

There are published investigations that show that, for malate oxidoreductases, substituting $\mathrm{NAD}^{+}$for $\mathrm{NADP}^{+}$can be used to alter the $K_{\mathrm{M}}$ for malate. In an attempt to increase TmME's $K_{\mathrm{M}}$ for malate, the assays described above were repeated with $1 \mathrm{mM} \mathrm{NADP}{ }^{+}$. The initial velocity increased up to $20 \mathrm{mM}$ malate, though using higher concentrations resulted in a 
progressive decrease in activity, somewhat similar to the kinetic behaviour of AcME. At 30 $\mathrm{mM}$ malic acid, activity had decreased to $75 \%$ of the maximum, and further decreased to $24 \%$ when using $40 \mathrm{mM}$. An approximate 4-fold increase in the $K_{\mathrm{M}}^{\text {app }}(8.0 \mathrm{mM})$ occurred, and 11-fold decrease in $k_{\text {cat }}{ }^{\text {app }}\left(0.4 \mathrm{~s}^{-1}\right)$.

Assays were not performed in the absence of the divalent metal ion, so it cannot be said for certain that TmME is a malic enzyme. However because of the co-factor preference for $\mathrm{NAD}^{+}$, TmME may belong to the EC 1.1.1.39 class.

Assays were repeated again, this time lowering the temperature to reflect the conditions used by the hypothetical end user of a biosensor. At $25^{\circ} \mathrm{C}$, and using $\mathrm{NAD}^{+}$as the cofactor, the apparent $k_{\text {cat }}$ decreased 3-fold to $1.5 \mathrm{~s}^{-1}$, while the apparent $K_{\mathrm{M}}$ increased to $2.0 \mathrm{mM}$ (Figure 2.10).

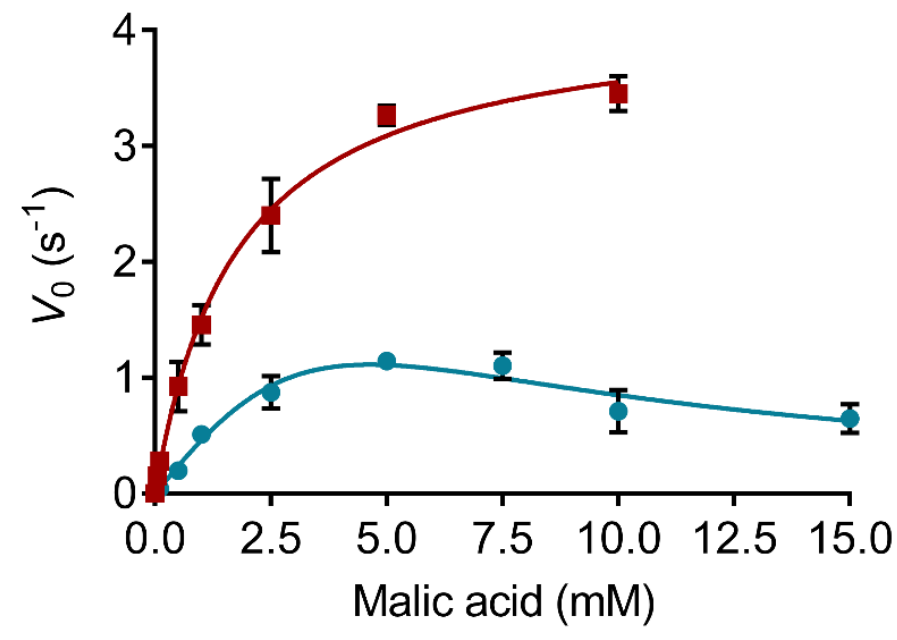

Figure 2.10. The kinetic behaviour of TmME at different temperatures, using $\mathrm{NAD}^{+}$as the co-factor. The initial velocity $\left(V_{0}\right)$ is plotted against increasing concentrations of malate. Assays were performed at $25^{\circ} \mathrm{C}$ (blue) and $50^{\circ} \mathrm{C}$ (red). Each data point shows the mean of triplicate repeats, with bars representing standard error. The data obtained at $50^{\circ} \mathrm{C}$ data has been fitted with the Michaelis-Menten equation, while the Haldane model for substrate inhibition was used for the data collected at $25^{\circ} \mathrm{C}$.

Similar to the kinetics observed for $\mathrm{NADP}^{+}$-based assays at $50^{\circ} \mathrm{C}$, activity decreased above a certain concentration of malic acid, though in this case the decline occurred at lower concentrations, starting at $10 \mathrm{mM}$ (figure 2.10 ). Assays at $25^{\circ} \mathrm{C}$ that used $\mathrm{NADP}^{+}$showed only trace activity, preventing apparent Michaelis-Menten parameters from being calculated. 
As the substrate inhibition at low temperatures limited the useable range of malic acid concentrations for a sensor, TmME was removed from the screening process.

\subsubsection{ApMDH and MaeA}

ApMDH was chosen as candidate for its reported tolerance to acidic conditions. However, it is not clear if this tolerance refers to ApMDH activity being resistant to changes in the $\mathrm{pH}$ used in assays, or if activity is simply retained in storage under acidic conditions (Kawakami et al., 2009). The assays described below sought to clarify this uncertainty by showing the $\mathrm{pH}$-dependence of enzyme activity.

The $\mathrm{pH}$ dependence of MaeA is also described here. While the $K_{\mathrm{M}}, 0.66 \mathrm{mM}$, is undesirable for this project, MaeA may still be useful if it is acid-tolerant. While an optimum $\mathrm{pH}$ has been reported, $\mathrm{pH} 7.5$, the enzyme's performance in the mildly acidic conditions have not been published.

Before ApMDH and MaeA had been successfully expressed and purified, crude $\mathrm{pH}$ dependence assays were performed using TmME and AcME. The assays used a range of buffers to ensure that reactions were buffered effectively at each $\mathrm{pH}$ tested. These were selected on the basis of having overlapping buffering ranges, allowing the effect of buffer type on enzyme activity to be compared at certain $\mathrm{pH}$ values. However, certain buffers inhibited enzyme activity (e.g. citrate buffer inhibited TmME), and it became clear that the best strategy for determining $\mathrm{pH}$ optima was to simply use potassium phosphate buffers for every $\mathrm{pH}$, as it produced superior enzyme velocities compared to other buffers in overlapping $\mathrm{pH}$ regions..

Using phosphate buffers, the $\mathrm{pH}$ dependence of ApMDH activity was tested over the range of pH $3-10$ (Figure 2.11a). The highest activity was observed at pH 10, which sharply decreased as the $\mathrm{pH}$ tended toward more acidic values. At $\mathrm{pH} 5$, the activity was $4.5 \%$ of that seen at $\mathrm{pH} 10$, and at $\mathrm{pH} 4$ only trace activity could be observed, which could not be measured reliably. The enzyme was chosen for the potential to create a device able to operate in undiluted grape juice ( $\mathrm{pH} 3-4)$. Due to activity being severely diminished at values $<\mathrm{pH}$ 4, ApMDH was not taken forward for future study. 
The $\mathrm{pH}$ dependence of MaeA activity was investigated over the range of $\mathrm{pH} 3-7.5$. Activity decreased from the $\mathrm{pH}$ optimum of 7 as the $\mathrm{pH}$ became more acidic (Figure 2.11b). Rates could be measured at $\mathrm{pH} 4$, though no activity was seen below this value. Like ApMDH, the $\mathrm{pH}$ dependence of MaeA was deemed unlikely to be useful for a biosensor used with grape juice or wine.
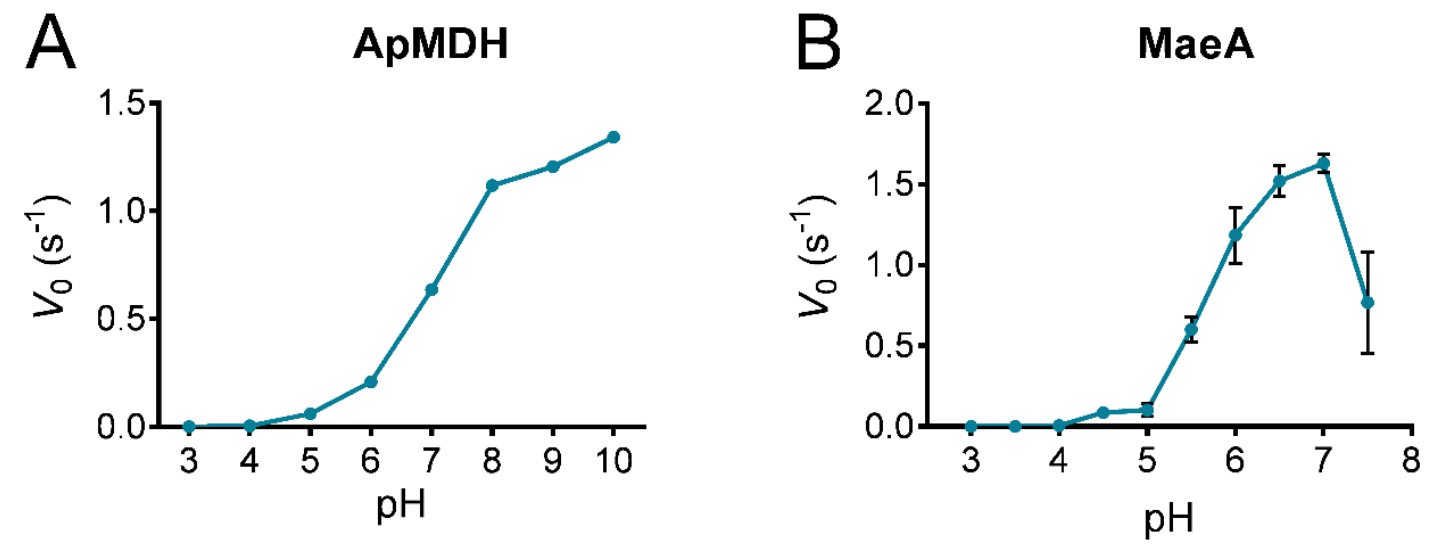

Figure 2.11. The activity of $\mathbf{A}) \mathrm{ApMDH}$ and $\mathbf{B}$ ) MaeA at different $\mathrm{pH}$ values, using potassium phosphate buffers. At each $\mathrm{pH}$, activity was measured as the initial velocity $\left(V_{0}\right)$. Points represent the mean of technical triplicates for ApMDH and duplicates for MaeA. Error bars, which represent standard error, are often too small to be seen.

\subsubsection{4. $\operatorname{AsME}(\mathrm{R} 181 \mathrm{Q})$ - the lead candidate}

The R181Q mutant of the A. suum malic enzyme was chosen for the high $K_{\mathrm{M}}$ reported by Karsten and Cook (2007), $57 \mathrm{mM}$, as well as the ability to modify this $K_{\mathrm{M}}$ through the addition of ammonium, potentially affording a biosensor with modifiable operational parameters. For the reasons described below, AsME(R181Q) emerged as the lead candidate for constructing a biosensor. This section describes the experiments that led to its selection for further characterisation in subsequent chapters.

As a malic enzyme, AsME(R181Q)'s activity is dependent on the presence of a divalent metal ion. Karsten and Cook (2007) used $\mathrm{Mg}^{2+}$ at saturating concentrations to determine the Michaelis-Menten kinetics, though the exact concentrations used in assays are not described. I therefore sought to characterise the magnesium dependence of $\operatorname{AsME}(\mathrm{R} 181 \mathrm{Q})$ to determine 
the optimum concentration (Figure 2.12). No activity was observed in the absence of $\mathrm{Mg}^{2+}$, though the initial velocity increased rapidly as its concentration was increased to a peak at 25 $\mathrm{mM}$. Further increases in $\left[\mathrm{Mg}^{2+}\right]$ resulted in a reduction of activity: at $200 \mathrm{mM}$, the rate is approximately $30 \%$ that seen at the peak. The decrease in rate appears to be biphasic, with an initial rapid decline as $\mathrm{Mg}^{2+}$ is increased to $100 \mathrm{mM}$, with the further increase to $200 \mathrm{mM}$ having less of an effect.

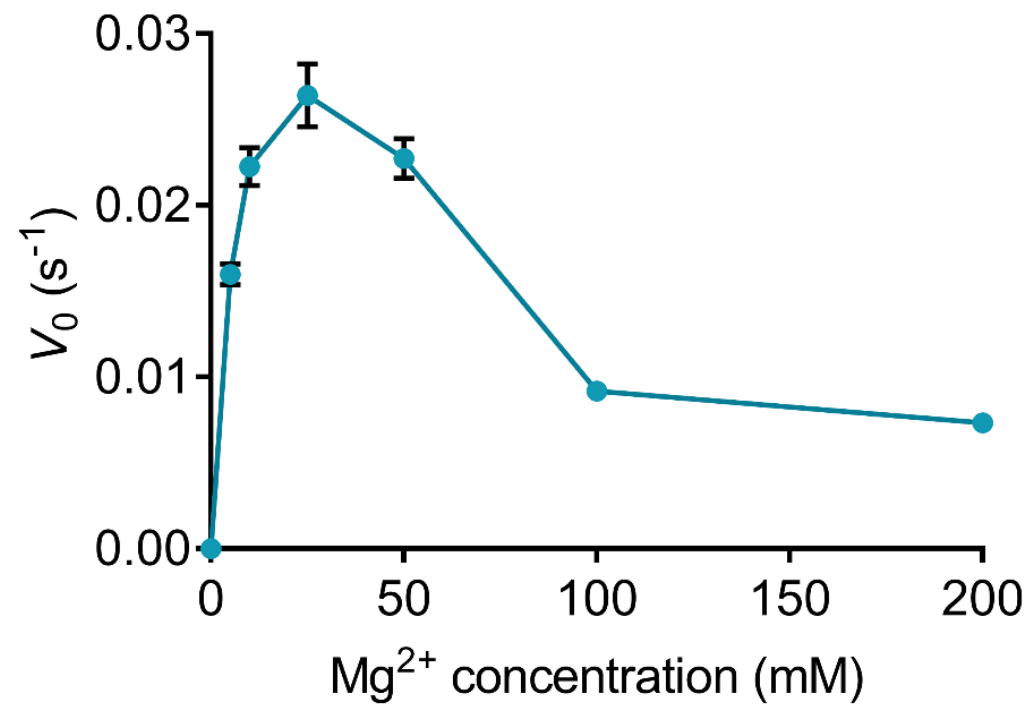

Figure 2.12. $\mathrm{Mg}^{2+}$ dependence of $\operatorname{AsME}(\mathrm{R} 181 \mathrm{Q})$. The initial velocity $\left(V_{0}\right)$ is plotted against increasing concentrations of $\mathrm{Mg}^{2+}$. Assays used a fixed concentration of $1 \mathrm{mM} \mathrm{NAD}^{+}$and $100 \mathrm{mM}$ malate. Each point is the mean of technical triplicates, with error bars showing the standard error - though these are often too small to be seen.

Subsequent assays sought to investigate the effect of various concentrations of ammonium on enzyme kinetics in the context of potentially creating a device with operational parameters that can be dynamically modified to suit the needs of the end user. Following previous work ((Karsten and Cook, 2007) and Table 2.4), assays in the presence of 0,4 , and $300 \mathrm{mM} \mathrm{NH}_{4}{ }^{+}$ were compared. Reaction mixtures initially used $25 \mathrm{mM} \mathrm{Mg}^{2+}$, as well as $10 \mathrm{mM} \mathrm{NAD}^{+}$, which was the optimized concentration for assays using each concentration of ammonia (assay plots shown appendix 1). The change in the initial velocities as the concentration of malate was increased is shown in Figure 2.13. 

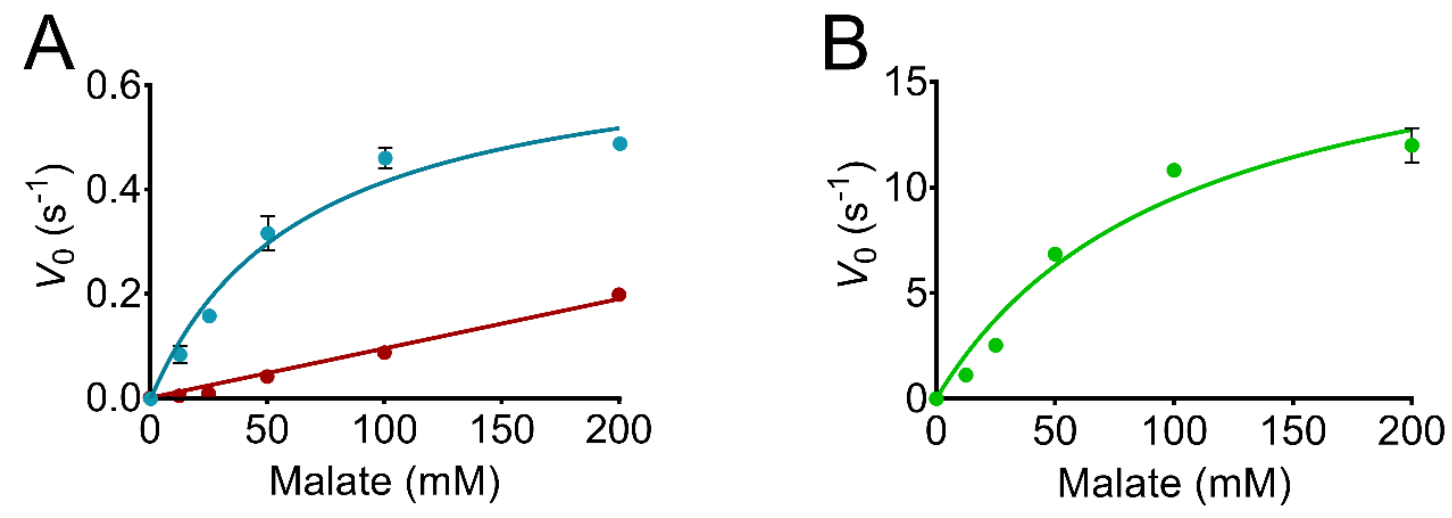

Figure 2.13. The kinetic behaviour of $\operatorname{AsME(R181Q)~at~different~concentrations~of~ammonium.~Assays~used~} 25$ $\mathrm{mM} \mathrm{Mg}^{2+}$ and $10 \mathrm{mM} \mathrm{NAD}^{+}$. The initial velocity $\left(V_{0}\right)$ is plotted against increasing concentrations of malate when using $\mathbf{A}$ - red) $0 \mathrm{mM} \mathrm{NH}_{4}{ }^{+}$, $\mathbf{A}$ - blue) $4 \mathrm{mM} \mathrm{NH}_{4}{ }^{+}$or $\mathbf{B}$ - green) $300 \mathrm{mM} \mathrm{NH}_{4}{ }^{+}$. Plotted points have been fitted with the Michaelis-Menten equation, with each point representing the mean of technical triplicates and error bars showing the standard error. Some error bars are too small to be seen.

Due to saturation kinetics not being fully observed, calculated Michaelis-Menten parameters were deemed "apparent". For assays using $0 \mathrm{mM} \mathrm{NH}_{4}{ }^{+}$, the $K_{\mathrm{M}}$ for malate could not be calculated, though the initial velocity increased linearly $\left(\mathrm{R}^{2}=0.99\right)$ for the full range of concentrations tested, $0-200 \mathrm{mM}$ malate. Adding $4 \mathrm{mM} \mathrm{NH}_{4}{ }^{+}$resulted in a $K_{\mathrm{M}}$ for malate of $65 \mathrm{mM}$ and a $k_{\text {cat }}$ of $6.9\left(\mathrm{~s}^{-1}\right)$. Initial velocities were generally 6 -fold greater than at $0 \mathrm{mM}$ $\mathrm{NH}_{4}{ }^{+}$. Using $300 \mathrm{mM} \mathrm{NH}_{4}{ }^{+}$further increased the size of the initial velocity, approximately 110 -fold greater than assays without $\mathrm{NH}_{4}{ }^{+}$. The $K_{\mathrm{M}}$ and $k_{\text {cat }}$ was $103 \mathrm{mM}$ and $19 \mathrm{~s}^{-1}$, respectively.

Crude assays showed that these $K_{\mathrm{M}}$ values could be increased by increasing the $\mathrm{Mg}^{2+}$ concentration. Due to the potential for increased $\mathrm{Mg}^{2+}$ chelation, which sequesters reagents, $\mathrm{NAD}^{+}$optimisation experiments were repeated in the presence of $200 \mathrm{mM} \mathrm{MgCl} 2$ for assays using 4 and $300 \mathrm{mM} \mathrm{NH}_{4}{ }^{+}$. These are shown in Figure 2.14. At both concentrations of $\mathrm{NH}_{4}{ }^{+}$, initial increases in $\mathrm{NAD}^{+}$result in greater velocities, following a hyperbolic trend resembling Michaelis-Menten-like behaviour. However, inhibition was observed beyond $20 \mathrm{mM} \mathrm{NAD}^{+}$

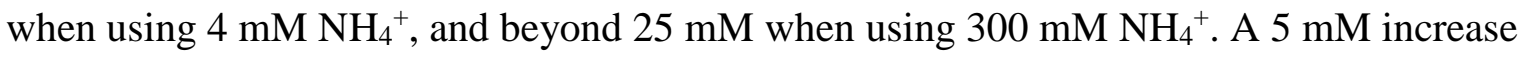
beyond each of these $\mathrm{NAD}^{+}$concentration 'limits' resulted in a subsequent $51 \%$ and $12 \%$ decreases in activity for $4 \mathrm{mM}$ and $300 \mathrm{mM} \mathrm{NH}_{4}{ }^{+}$assays, respectively. 

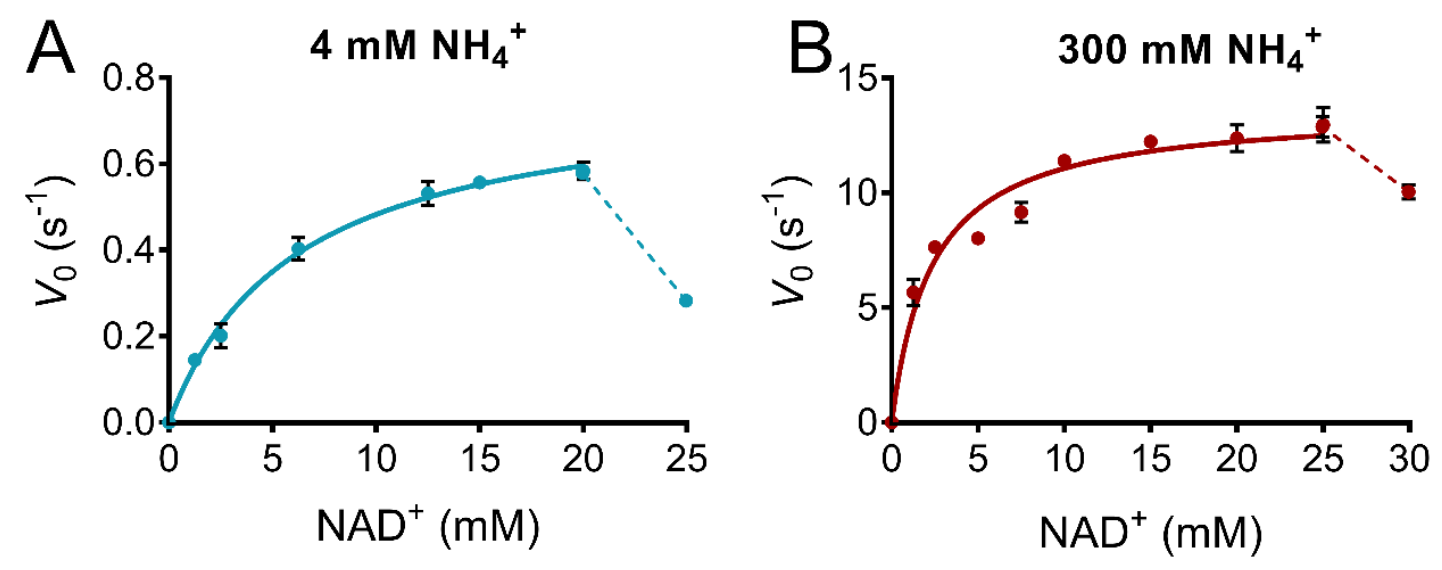

Figure 2.14. The kinetic behaviour of $\operatorname{AsME}(\mathrm{R} 181 \mathrm{Q})$ at different concentrations of $\mathrm{NAD}^{+}$, when using $200 \mathrm{mM}$ $\mathrm{Mg}^{2+}$ in assays. The initial velocity $\left(V_{0}\right)$ is plotted against increasing concentrations of $\mathrm{NAD}^{+}$when using A) 4 $\mathrm{mM}$ and B) $300 \mathrm{mM} \mathrm{NH}_{4}^{+}$in assays. Assays used a fixed concentration of $100 \mathrm{mM}$ malate. Plots have been fitted with the Michaelis-Menten equation, with each point representing the mean of triplicates. Error bars show the standard error, though are often too small to be seen.

Kinetics assays that varied malate concentrations were repeated using $200 \mathrm{mM} \mathrm{MgCl}_{2}$ and optimised NAD ${ }^{+}$concentrations: $20 \mathrm{mM} \mathrm{NAD}^{+}$when using $4 \mathrm{mM} \mathrm{NH}_{4}{ }^{+}$(Figure 2.14a), and $25 \mathrm{mM}$ when using $300 \mathrm{mM} \mathrm{NH}_{4}{ }^{+}$(figure 2.14b). Again, saturation kinetics were not observed over the $0-200 \mathrm{mM}$ malate tested (Figure 2.15), making calculated values “apparent". It was not possible to calculate the Michaelis-Menten parameters for plots that used $4 \mathrm{mM} \mathrm{NH}_{4}{ }^{+}$, though the curve was highly linear over the full range of malate concentrations $\left(\mathrm{R}^{2}=0.99\right)$. Assays using $300 \mathrm{mM} \mathrm{NH}_{4}{ }^{+}$, produced apparent $K_{\mathrm{M}}$ and $k_{\text {cat }}$ values of $540 \mathrm{mM}$ and $48 \mathrm{~s}^{-1}$, respectively. Both values were far greater than the corresponding assays using $25 \mathrm{mM} \mathrm{Mg}^{2+}$. The plot was subsequently fitted to the equation of a straight line, showing the increase in initial velocity to also be highly linear $\left(\mathrm{R}^{2}=0.99\right)$. 


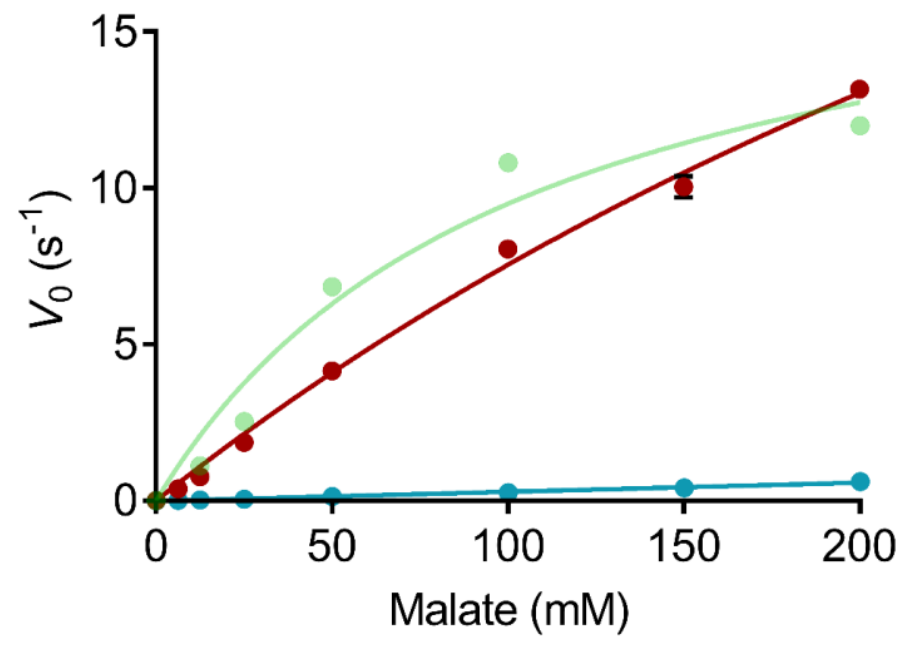

Figure 2.15. The kinetic behaviour of $\operatorname{AsME}(\mathrm{R} 181 \mathrm{Q})$ at different concentrations of malate, when using $200 \mathrm{mM}$ $\mathrm{Mg}^{2+}$ in assays. The initial velocity $\left(V_{0}\right)$ is plotted against increasing concentrations of malate when using (blue) $4 \mathrm{mM}$ and (red) $300 \mathrm{mM} \mathrm{NH}_{4}^{+}$in assays. The data from Figure 2.13b (300 $\mathrm{mM} \mathrm{NH}_{4}^{+} ; 25 \mathrm{mM} \mathrm{Mg}^{2+}$ ) have been overlaid in pale green. Plots have been fitted with the Michaelis-Menten equation, with each point representing the mean of triplicates. Error bars show the standard error, though are often too small to be seen.

Overall, these experiments indicated that $\operatorname{AsME(R181Q)~had~potential~for~use~in~a~biosensor~}$ because its initial velocity did not saturate, even at the very high concentrations of malate (200 $\mathrm{mM})$ that can be found in ripening wine grapes.

\subsection{Discussion}

The goal of the work in this chapter was to identify malate oxidoreductases with $K_{\mathrm{M}}$ values much higher than those of the commercial enzymes. We hypothesised that these could be used to create a malate biosensor with a wide linear range.

The search was successful: the three high- $K_{\mathrm{M}}$ variants chosen from BRENDA had reported values of 12, 27.6 and $57 \mathrm{mM}, 30-, 69$ - and 140-fold greater than the $K_{\mathrm{M}}$ of the porcine $\mathrm{MDH}$, the most commonly used enzyme for malate biosensors (Table 2.1).

BRENDA not only holds vast numbers of $K_{\mathrm{M}}$ values, but also curates many other functional parameters and molecular properties for each enzyme. This allowed a secondary selection 
scheme to quickly develop in the early stages of the search which focused on finding enzymes that offer the potential for extra utility in a biosensor.

Such utility may have included being able to function in the acidic conditions of grape juice/wine (ApMDH) or a mechanism to switch activity on or off when desired (SvMDH). Using an enzyme from the malate dehydrogenase (quinone) class (EC 1.1.5.4) was also considered to remove the need for solubilised $\mathrm{NAD}(\mathrm{P})^{+}$. In these enzymes, $\mathrm{NAD}(\mathrm{P})^{+}$is replaced by a tightly bound FAD co-factor integrated into the enzyme's structure (Kather et $a l ., 2000)$. However, this type of utility has already been used in malate biosensors, and accordingly, the focus shifted to finding enzyme characteristics that had the potential to afford novel functionality.

Particularly high value was placed on enzymes whose kinetics could be modified through the use of specific compounds. The $K_{\mathrm{M}}$ of both AcME and AsME(R181Q) can be modulated between high and low values, and we envisioned a device with a sensitivity versus linear range trade-off, that is, a device that whose sensitivity can be increased by using the low $K_{\mathrm{M}}$ form of the enzyme, though at the cost of the linear range, and the ability to switch to the high $K_{\mathrm{M}}$ form, which is less sensitive but has a wider linear range. Such a sensor would suit the needs of winemakers who want to measure small concentrations of malic acid, such as those in malolactic fermentation, and large concentrations, such as those found within the maturing grape. No such switchable mechanism has been described for malate sensors; nor, to the best of our knowledge, the enzyme-based biosensor field in general. Certain commercial malic acid testing kits are sold as 'normal' and 'extended-range' models (https://www.unitechscientific.com/), though the need to buy two kits could be mitigated by using a biosensor with a dynamically tuneable linear range.

A summary of the screening procedure is shown in Table 2.5. The aim was not to comprehensively (re)characterise each enzyme, but rather to rapidly identify a lead candidate for biosensor development. For this reason, troublesome enzymes were quickly removed from the screening process, though this is not to say that these enzymes are unusable. With further work, rejected candidates may still be implemented into a sensor. For example, though significant efforts were made to express $\mathrm{SvMDH}$ as a soluble protein, other strategies to improve solubility remain to be tested, including the use of detergents, reducing agents and a wider-ranging buffer screen (Golovanov et al., 2004). For AcME, which aggregated on ice, the stability may be improved using additives such as glycerol (Vagenende et al., 2009). 
However, including such endeavours would make the screening process much longer and commercial implementation will ultimately require enzymes that are easy to produce and store.

Table 2.5. Summary of the screening process

Malate oxidoreductase

AcME

ApMDH

SVMDH

TmME

MaeA
Reason for exclusion

Unstable on storage

pH-stable activity not observed

Unable to be expressed as soluble protein

Substrate inhibition at $>5 \mathrm{mM}$ malic acid

Low $K_{\mathrm{M}}$

Non-Michaelis-Menten behaviour was observed for TmME and AcME. For the latter, the activity decreases as malate concentration is increased beyond $12.5 \mathrm{mM}$, though the trend fits poorly to the model of substrate inhibition (Figure 2.9). Such kinetic behaviour was not observed by (Zhang et al., 2012). This difference may be the result of assays in my study using CoA, instead of the acetyl-CoA: a comparison of each molecule's effects on the Michaelis-Menten kinetics was not pursued due to AcME being removed from the screening process for its instability. Conversely, no previously published kinetic data for TmME has been described to allow for comparison. Substrate-inhibited malate oxidoreductases jeopardise the accuracy of a biosensor, as two concentrations of malate can produce the same signal. Their existence, shown here and in other studies (Ge et al., 2010), means that the kinetic behaviour of future candidates should be tested at high concentrations of malate as part of the screening procedure. 
The lead candidate, $\operatorname{AsME}(\mathrm{R} 181 \mathrm{Q})$, was chosen for its kinetic behaviour, with the activity being shown here to increase linearly up to high concentrations of malate. The extent of these linear ranges depends on the concentrations of both $\mathrm{Mg}^{2+}$ and $\mathrm{NH}_{4}{ }^{+}$present in assays.

Karsten and Cook (2007), who first characterised the kinetics of AsME(R181Q), reported a $K_{\mathrm{M}}$ of $57 \mathrm{mM}$, though this reflects the concentration of malate corrected for the effects of magnesium chelation - AsME(R181Q) cannot use malate when complexed with magnesium. Karsten and Cook (2007) do not provide sufficient information to reversecalculate the $57 \mathrm{mM} K_{\mathrm{M}}$ to the "total" amount of malate present in assays (Park et al., 1984) the format used in my study - making it impossible to compare to the malate-dependent kinetics.

However, certain other trends can be compared, and indeed, are similar: like the results presented by Karsten and Cook (2007), varying the $\mathrm{NH}_{4}{ }^{+}$concentration used in assays can be used to modulate the enzyme kinetics. For my assay mixtures, that contained $25 \mathrm{mM} \mathrm{Mg}^{2+}$, using $300 \mathrm{mM} \mathrm{NH}_{4}{ }^{+}$results in initial velocities over 100-fold greater than assays without $\mathrm{NH}_{4}{ }^{+}$, though the enzyme begins to saturate at lower concentrations (Figure 2.13). This is consistent with the previously published trend that $300 \mathrm{mM} \mathrm{NH}_{4}{ }^{+}$increases the $k_{\text {cat }}$ while decreasing the $K_{\mathrm{M}}$.

However, this mode of tuning became somewhat redundant after changing the $\mathrm{Mg}^{2+}$ concentration from 25 to $200 \mathrm{mM}$. With the increased magnesium, using $300 \mathrm{mM} \mathrm{NH}_{4}{ }^{+}$, the initial velocities were not only similar to those in the plot of those in the $300 \mathrm{mM} \mathrm{NH}_{4}{ }^{+} / 25$ $\mathrm{mM} \mathrm{Mg}{ }^{2+}$ assays, but the apparent $K_{\mathrm{M}}$ also increased from 103 to $540 \mathrm{mM}$, meaning saturation kinetics were not observed even at $200 \mathrm{mM}$ malic acid, the maximum concentration seen in grapes. It is unclear to what extent this increase kinetic behaviour is the product of the extra magnesium increasing the $K_{\mathrm{M}}$, or the effect of magnesium sequestering malate, thereby increasing the apparent $K_{\mathrm{M}}$. In the following chapter, this information on how $\mathrm{Mg}^{2+}$ and $\mathrm{NH}_{4}{ }^{+}$, concentrations alter AsME(R181Q) activity was used to inform the design of biosensor prototypes. 


\section{Chapter Three - Creating a working amperometric biosensor}

\subsection{Chapter introduction}

In chapter two, the R181Q mutant of Ascaris suum ME was chosen as the enzyme to be used in the biosensor. The work in this chapter focused on the translation of the enzymatic production of NADH into measurable electrical current with the aim of obtaining a wide linear range from the kinetics shown in spectrophotometric assays. The notion of creating a biosensor with a tuneable linear range versus sensitivity trade-off was also revisited.

\subsubsection{Electron mediators}

When measuring an analyte in a complex solution such as grape juice or wine, amperometric biosensors generally have been designed to use the lowest potential possible. This is to avoid unwanted electron-transfer reactions involving the myriad of biological compounds, which may generate non-specific current signals (Bucur et al., 2006, Giménez-Gómez et al., 2017, Lupu et al., 2004). However, for many dehydrogenase-based biosensors, one limitation is that a high potential is required to oxidise NADH (Prieto-Simón and Fàbregas, 2004). It is therefore now standard practice to incorporate an electron mediator into the biosensor architecture, which acts to lower the required potential, as well as increasing the output signal by improving electron-transfer kinetics (Nagels and Staes, 2001). In my comprehensive survey (section 1.5; Matthews et al. 2021), I found that 18 of the 20 most recent malate biosensors have incorporated an electron mediator into the architecture, including the sensors that use carbon nanotube modified electrodes to achieve the same effect.

A wide range of electron mediators have been described in the literature, typically in the form of inorganic redox ions or organic dyes (Giménez-Gómez et al., 2017). The impact on a biosensor, in terms of lowering the potential and increasing sensitivity, varies between mediators, and is highly dependent on the sensor architecture itself. For example, many of the 
organic dyes change protonation states when transferring electrons, making their performance dependent on the $\mathrm{pH}$. Some mediators may interact poorly with NADH or certain electrode materials, and others facilitate unwanted electron-transfer reactions from interfering molecules (Tonomura et al., 1978). As such, it is important to screen a range of mediators for a given biosensor system and choose the one best suited for the final design.

\subsubsection{Techniques used to study the electrochemistry of biosensors}

The electrochemical techniques used in this project are based around a three-electrode system. This type of electrode employs working, counter and reference electrodes, which were used in conjunction to enable the measurement of electrical current at a set potential. The reference electrode is used by a potentiostat to maintain this desired potential, which is applied between the counter and working electrodes. The working electrode is the interface between the analyte in solution and the solid-state electronics such as wires and the ammeter used for measuring current. At an appropriate potential, redox reactions between the analyte and working electrode are made thermodynamically favourable, promoting electron transfer (Bard, 2001). The electrode material has profound effects on the size of the current generated, as some analytes interact poorly with certain materials e.g. oxygen has a poor interaction with carbon electrodes (Ucar et al., 2017). There also exists a directly proportional relationship between the working electrode's surface area and current, given by the Cottrell equation (Cottrell, 1903). Accordingly, all currents in this chapter have been standardised for the 0.13 $\mathrm{cm}^{2}$ electrode area used to develop biosensors, and therefore are reported as current density $\left(\mu \mathrm{A} \mathrm{cm}^{-2}\right)$.

\subsubsection{Cyclic voltammetry}

Within the electrochemist's repertoire of techniques, cyclic voltammetry serves as a powerful tool to study the redox behaviour of electroactive species. In this project, cyclic voltammetry has been used to determine the working potential required for signal generation.

The change in current is measured as the potential is cycled, or "swept", between a high and low potential at a defined rate. In a simple scenario, the cycle results in both sweep directions producing a current peak that corresponds to the reduction and oxidation of the species 
(Figure 3.1) (Elgrishi et al., 2018). Working potentials for amperometric sensors are chosen on the basis of which potentials these peaks are seen.

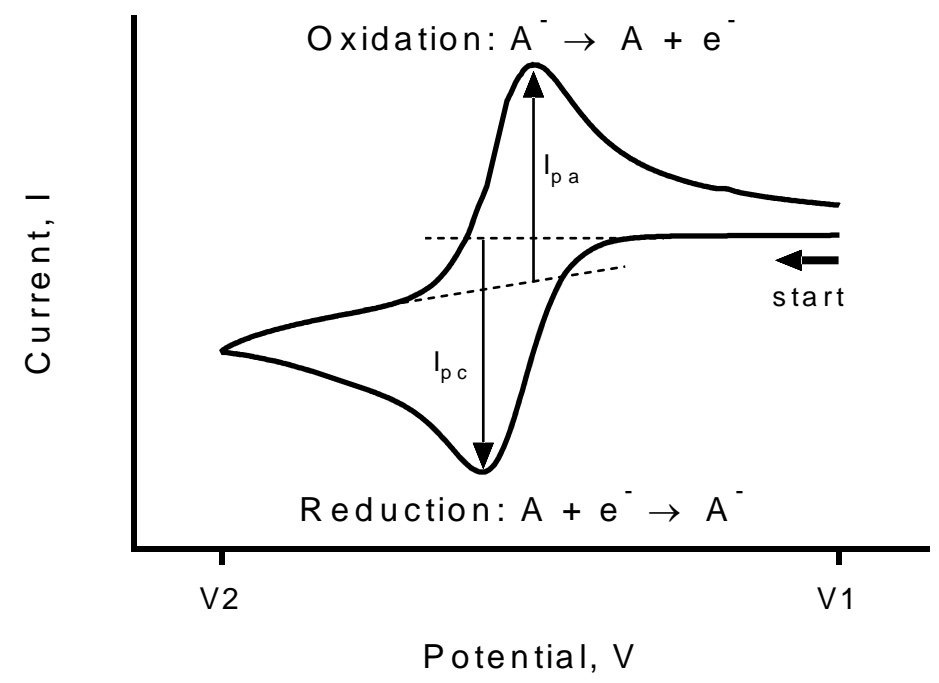

Figure 3.1. Example of a cyclic voltammogram for a reversible reaction. $I_{p a}$ and $I_{p c}$ represent the peak anodic and cathodic currents, respectively. The baseline current reading is extrapolated (dashed lines) to determine the peak size.

\subsubsection{Chronoamperometry}

After cyclic voltammetry has been used to determine the optimal potential, the enzyme-based biosensor then uses the principle of chronoamperometry. This is when a change in current is measured over time, at a set potential. A typical trace is shown in Figure 3.2. 


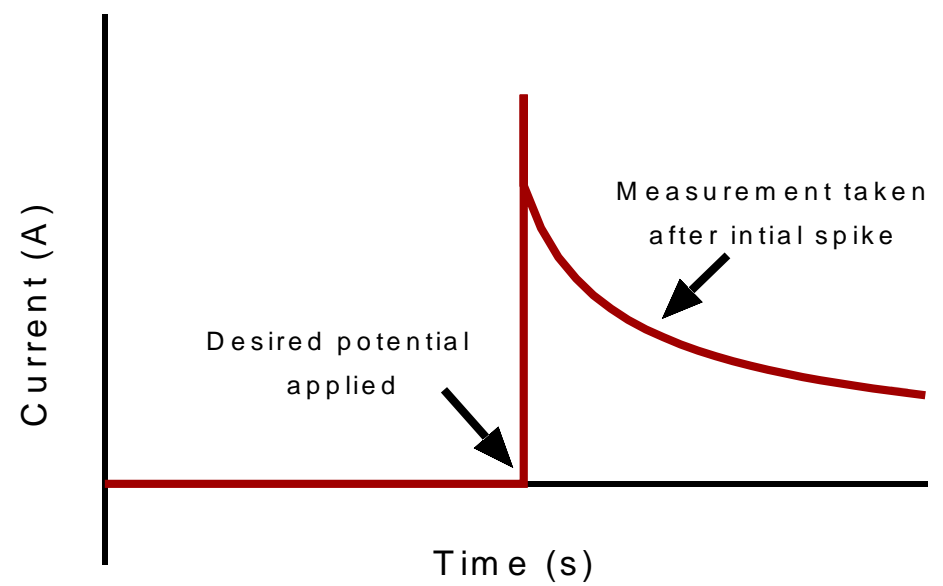

Figure 3.2. A typical chronoamperometry trace.

Like for cyclic voltammetry, a trend in decay is the product of an expanding diffusion layer as the analyte is depleted at the electrode surface. An initial spike occurs when potential is first applied. It represents the capacitive current caused by ions accumulating at the electrode surface, and quickly decays in the order of milliseconds. As this type of current is different from Faradaic current, caused by redox reactions between the electrode and the analyte, current measurements are taken at a set time a short while after the potential is first applied (Bard, 2001).

The work in this chapter sought to identify a suitable electron mediator and working potential, then create a working biosensor assay using chronoamperometry. Experiments were designed to optimise these aspects of biosensor construction.

\subsection{Results}

\subsubsection{Screening electron mediators}

Six different mediators were tested for their suitability in our biosensor system, with two being promptly eliminated from the screen. The mediator phenazine methosulfate was unstable in the buffer used for enzyme assays (100 mM HEPES pH 7), as the solution rapidly changed colour from yellow to dark brown. Another mediator, methyl red, was insoluble in this buffer. 
Cyclic voltammetry traces were generated for the remaining four mediator candidates (Figure 3.3). Without a mediator, the oxidation peak for NADH was seen at the relatively high potential of $750 \mathrm{mV}$. This was consistent with the values reported for NADH oxidation in other buffers (Arvinte et al., 2008, Blandón-Naranjo et al., 2018) and emphasised the need to use a mediator to lower the peak oxidation potential and avoid non-specific oxidation of wine components.

The lowest peak oxidation potential was seen using hexaammineruthenium(III) chloride (HAR) at $-150 \mathrm{mV}$, followed by gallocyanine at $-130 \mathrm{mV}, 2,6$-dichlorophenolindophenol (DCPIP) at $+30 \mathrm{mV}$, and finally potassium ferricyanide at $+210 \mathrm{mV}$. Shown in Table 3.1, selected working potentials were generally $+100 \mathrm{mV}$ greater than each peak oxidation potential, with care being taken to avoid potentials that existed in the regions of both reduction and oxidation peaks, as this caused current readings to become highly variable. 

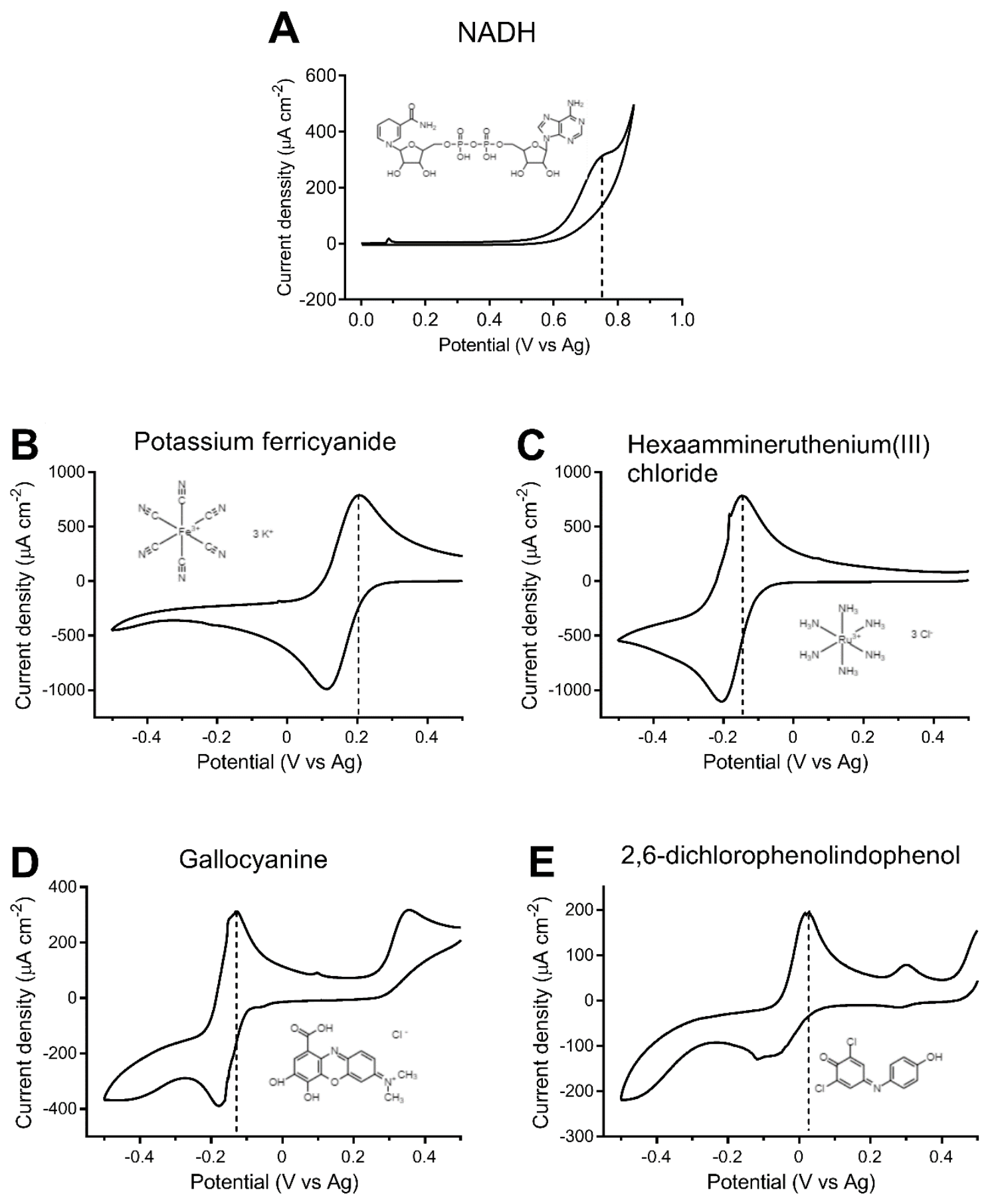

Figure 3.3. Cyclic voltammograms of NADH and various electron mediators: A) NADH B) potassium ferricyanide C) hexaammineruthenium(III) chloride D) 2,6-dichlorophenolindophenol. Each trace was generated using 5 $\mathrm{mM}$ of the compound dissolved in $100 \mathrm{mM}$ HEPES buffer $\mathrm{pH} 7$, containing $300 \mathrm{mM} \mathrm{NH}_{4}{ }^{+}$and $200 \mathrm{mM} \mathrm{Mg}^{2+}$ to replicate the conditions of the activity assay for $\operatorname{AsME}(\mathrm{R} 181 \mathrm{Q})$. Anodic peak currents that were used to select the working potentials of each mediator is shown by a dashed line. The scan rate was $100 \mathrm{mV} / \mathrm{s}$. 
Table 3.1. Performance of tested electron mediators. Assays used $5 \mathrm{mM}$ of both NADH and the mediator. The values given for the net analytical signal are the mean of technical triplicates, with the variability representing standard error.

\begin{tabular}{ccc} 
Mediator & Working potential $(\mathbf{m V})$ & $\begin{array}{c}\text { Net analytical signal } \\
\left(\mu \mathrm{A} \mathrm{cm} \mathbf{~ c m}^{-2}\right)\end{array}$ \\
HAR & +50 & $16( \pm 1)$ \\
Gallocyanine & +50 & $4.0( \pm 0.2)$ \\
DCPIP & +150 & $29( \pm 1)$ \\
\hline
\end{tabular}

HAR = Hexaammineruthenium(iii) chloride, DCPIP = 2,6-Dichlorophenolindophenol

While it had the highest (and therefore least desirable) peak oxidation potential (Figure 3.3b), potassium ferricyanide has been used to create some of the most sensitive malate biosensors (Monošík et al., 2012a). However, during the cyclic voltammetry experiments, I observed electrode fouling as a brown deposit on the silver reference electrode (Figure 3.4). Control experiments showed that this deposition was dependent on the mediator being reduced in the presence of the magnesium that was required in the buffer to ensure enzyme activity. For this reason, potassium ferricyanide was ruled out for use in the biosensor.
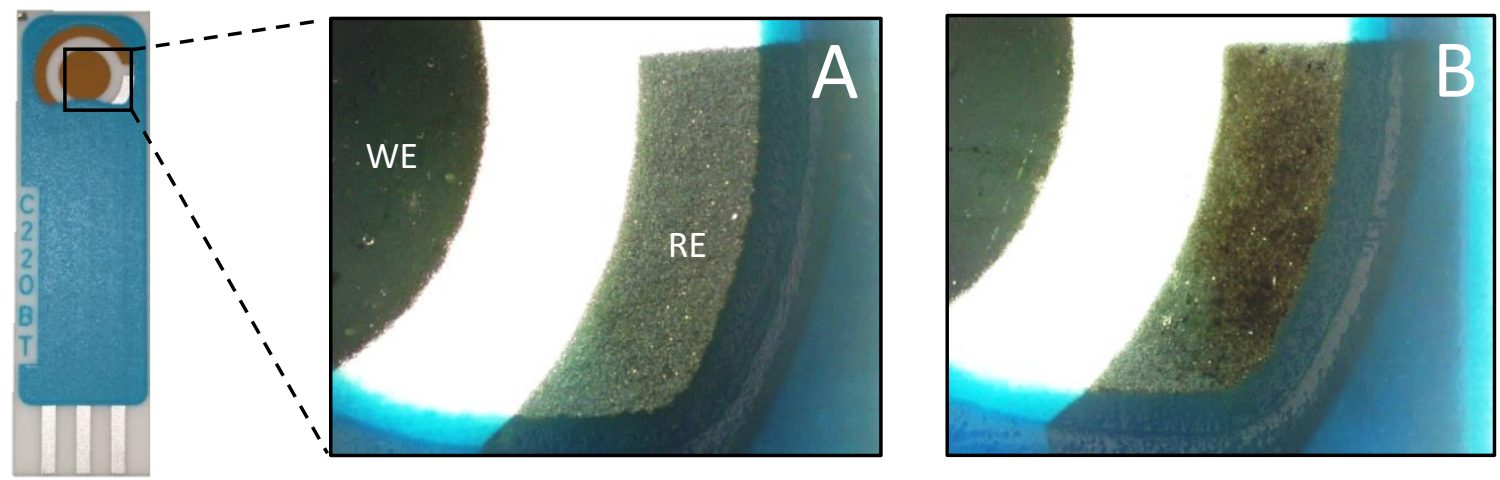

Figure 3.4. Reference electrode fouling when using ferricyanide. A) a new, untainted screen-printed electrode. The working electrode (WE) and reference electrode (RE) are labelled. B) electrode fouling in the form of a brown deposit on the reference electrode after being subjected to assays using both the mediator and magnesium. Images were taken under 10x magnification. 
The voltammograms showed that certain mediators produce greater peak oxidation currents than others (e.g. HAR versus DCPIP, Figure 3.3). However, the ability of each mediator to oxidise NADH remained unclear. The analytical signal was therefore measured in the presence of the cofactor at the chosen working potentials. Baseline readings in the absence of NADH were performed first: for DCPIP and gallocyanine, these readings took the form of small oxidative currents less than $0.05 \mu \mathrm{A}$, or $<5 \%$ of the net signal. The baseline of HAR, however, produced a large cathodic current $\left(-15 \mu \mathrm{A} \mathrm{cm}^{-2}\right)$. The greatest net analytical signal was seen using DCPIP, which is approximately 2-fold greater than that when using HAR and 8-fold greater than gallocyanine. DCPIP, in conjunction with its low operating potential, was therefore chosen to be taken forward in the development of the biosensor.

\subsubsection{Creation of a standard assay and mediator optimisation}

A standard assay was created using 10.2 $\mu \mathrm{M}$ AsME(R181Q), $10 \mathrm{mM} \mathrm{NAD}^{+}, 200 \mathrm{mM} \mathrm{MgCl}_{2}$, $300 \mathrm{mM} \mathrm{NH}_{4}{ }^{+}$and $100 \mathrm{mM}$ HEPES $\mathrm{pH}$ 7. A total of $50 \mu \mathrm{l}$ of the assay mixture was pipetted onto the screen-printed electrode (as shown in Figure 3.4a). The enzyme-catalysed reaction was started with the addition of malate and was allowed to proceed for two minutes before a potentiostat was used to apply the $+150 \mathrm{mV}$ potential. Current was measured after $5 \mathrm{~s}$. Initial assays allowed for a five-minute temperature equilibration step prior to starting the assays, though it was later found that removing this step left readings unaffected.

DCPIP, when dissolved in the buffer, formed a precipitate after approximately five min. The stock solutions of the mediator instead used water, and once mixed with the other reagents dissolved in HEPES, the biosensor assay was started within one min.

To find the optimal mediator concentration, a range of $0.1-0.5 \mathrm{mM}$ was tested in assays with a fixed concentration of $200 \mathrm{mM}$ malate. Increasing the DCPIP concentration caused the current response to also increase, following a shallow hyperbolic trend from $7.3 \mu \mathrm{A}$ to 27.7 $\mu \mathrm{A}$ (Figure 3.5), a four-fold difference. Higher concentrations were not tested due to the solubility limits of the stock solution. Future experiments then used 0.5 mM DCPIP. 


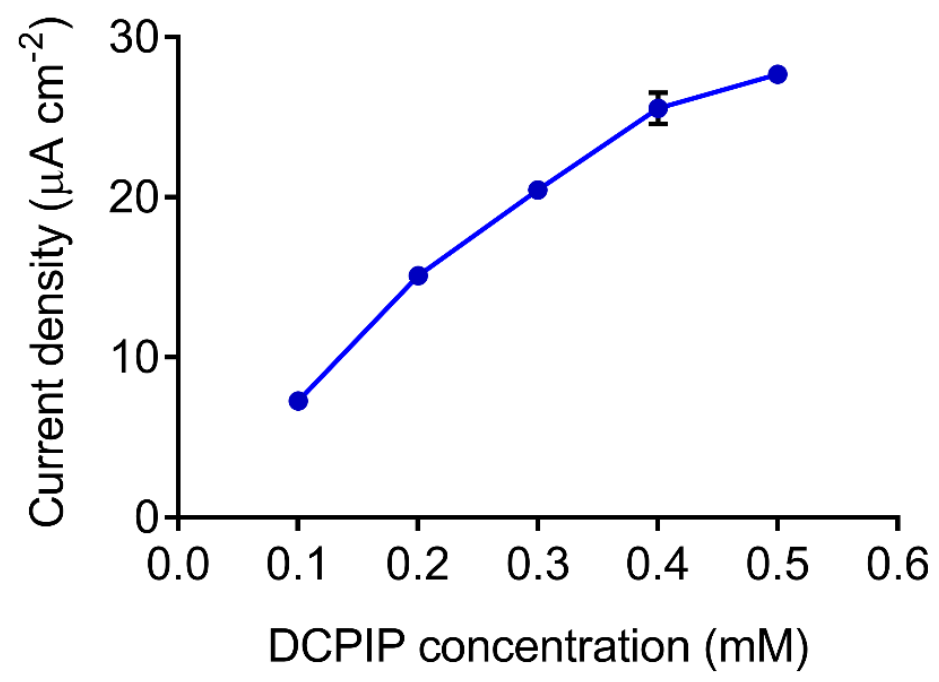

Figure 3.5. Optimisation of 2,6-dichlorophenolindophenol concentration used in biosensor assays. Plotted points show the mean of triplicates, with error bars representing standard error, though these are often too small to be seen.

\subsubsection{Biosensor \#1 - $\mathrm{Mg}^{2+}$ used as a co-factor}

Using the standard assay and optimised DCPIP concentration described in section 3.2.2, a calibration curve was constructed from 0 to $200 \mathrm{mM}$ malic acid (Figure 3.6). The curve is highly linear from $25-200 \mathrm{mM}$ with a sensitivity of $0.11 \mu \mathrm{A} \mathrm{mM} \mathrm{m}^{-1} \mathrm{~cm}^{-2}$; that is, each increase in malate concentration of $1 \mathrm{mM}$ yielded an increase in current density of $0.11 \mu \mathrm{A}$ $\mathrm{cm}^{-2}$. The blank, obtained in the absence of malic acid, gave a current reading of $1.2 \mu \mathrm{A} \mathrm{mM}^{-}$ ${ }^{1} \mathrm{~cm}^{-2}$ with a standard deviation of $0.12 \mu \mathrm{A} \mathrm{mM}^{-1} \mathrm{~cm}^{-2}$. The limit of detection (LOD), the lowest difference in analyte concentration a sensor is able to detect, was estimated by dividing $3 \times$ the standard deviation of the blank by the slope of the linear range. For biosensor $\# 1$, the LOD was $3.3 \mathrm{mM}$.

An unexpectedly high current was seen repeatedly at $10 \mathrm{mM}$ malic acid, $4.8 \mu \mathrm{A} \mathrm{mM}^{-1} \mathrm{~cm}^{-2}$, which is approximately $25 \%$ larger than that produced with $25 \mathrm{mM}, 3.8 \mu \mathrm{A} \mathrm{mM}^{-1} \mathrm{~cm}^{-2}$ (Figure 3.6). This 'spike' corresponds to $45 \mathrm{mM}$ on the linear calibration curve, making some concentrations on the linear range unusable. The usable lower limit of the linear range was therefore redefined as the smallest concentration of malate that produces a signal greater than that of the spike current (spike current + LOD). Accordingly, the useable linear-range is 48 $200 \mathrm{mM}$. 


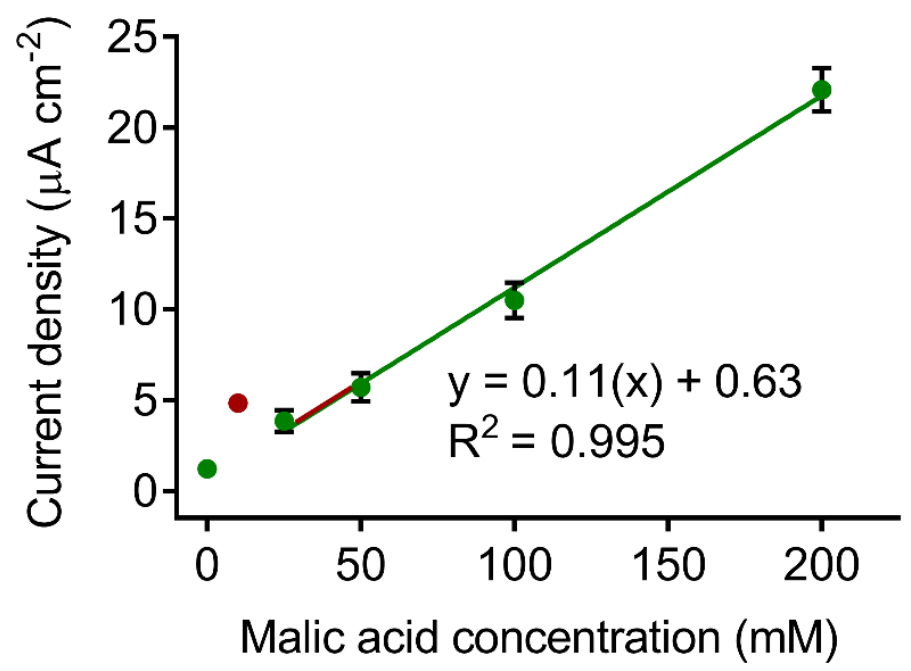

Figure 3.6. Calibration curve for biosensor \#1. The line of best fit (and associated $R^{2}$ ) is for the range $25-200$ $\mathrm{mM}$ malic acid. Plotted points are the mean of triplicates, with bars representing standard error, though these are sometimes too small to be seen. The red point highlights an unexpectedly high current observed at $10 \mathrm{mM}$ malic acid. The portion of the linear range made unusable by this "spike current" is also shown in red.

\subsubsection{Biosensor \#2 - $\mathrm{Mn}^{2+}$ used as a co-factor}

Previous studies have shown that for malic enzymes, the type of divalent metal ion used in assays, either magnesium or manganese, can have profound effects on the enzyme kinetics, including the $K_{\mathrm{M}}$ for malate (Lin and Davis, 1974, Casati et al., 1997).

The $200 \mathrm{mM}$ magnesium dication used in assays was substituted for the same concentration of $\mathrm{Mn}^{2+}$, and the change in the biosensor's performance was investigated. The resulting calibration curve showed a sensitivity of $0.57 \mu \mathrm{A} \mathrm{mM}^{-1} \mathrm{~cm}^{-2}-$ a 5-fold increase compared to biosensor \#1, though this was accompanied by a reduction in the linear range, to $5-50 \mathrm{mM}$ (Figure 3.7). The use of $\mathrm{Mn}^{2+}$ also increased the baseline noise, shown by the average reading of the blank increasing from 1.2 to $1.8 \mu \mathrm{A} \mathrm{mM}^{-1} \mathrm{~cm}^{-2}$. However, the estimated LOD is fourfold lower, at $0.85 \mathrm{mM}$, due to the increase in sensitivity.

It was noticed that solutions of $\mathrm{MnCl}_{2}$ dissolved in $100 \mathrm{mM}$ HEPES $\mathrm{pH} 7$ began to undergo an overnight colour change from a pink tint to brown, corresponding to manganese's oxidation to $\mathrm{Mn}^{3+}$ (Johnson and Stokes, 1966). In case this effected readings, measurements were made within 6 hours of the creation of the $\mathrm{MnCl}_{2}$ stock solution. 


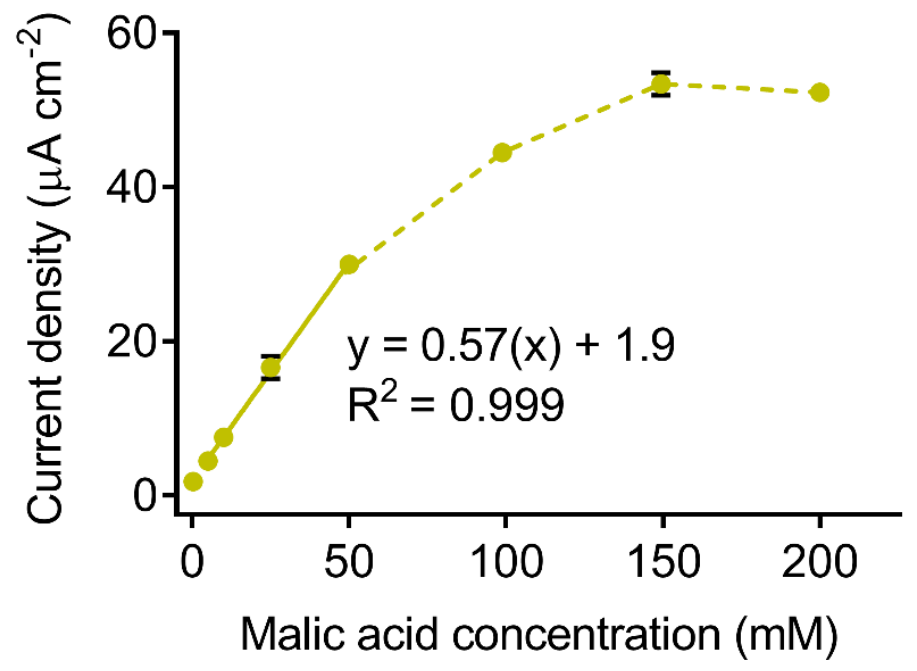

Figure 3.7. Calibration curve for biosensor \#2. The line of best fit (and associated $\mathrm{R}^{2}$ ) is for the range $5-50 \mathrm{mM}$ malic acid. Plotted points are the mean of triplicates, with bars representing standard error, though these are often too small to be seen.

\subsubsection{Biosensor \#3 $-\mathrm{Mn}^{2+}$ and citrate used together}

Various compounds found in wine were tested for any interfering effects. This investigation is described in more detail in Chapter 4, though citrate was found to amplify the signal. In an effort to create a device with even greater sensitivity than biosensor \#2, a third biosensor was created that used citrate and $\mathrm{Mn}^{2+}$ together.

First, the effect of increasing citrate concentration from $0-100 \mathrm{mM}$ was investigated (Figure 3.8), with the upper limit defined by the solubility limit of the stock solution. Increasing the concentration from 0 to $25 \mathrm{mM}$ yielded a 1.5 -fold increase in signal, with greater concentrations further increasing the current generated: at $100 \mathrm{mM}$ citric acid, the reading was 2.3-fold greater than the original reading. Adding $100 \mathrm{mM}$ citric acid to the assay components in biosensor \#1 $\left(\mathrm{Mg}^{2+}\right.$ rather than $\left.\mathrm{Mn}^{2+}\right)$ yielded a smaller increase, 1.5-fold, showing that a greater synergy between $\mathrm{Mn}^{2+}$ and citrate exists for generating the greatest signals. 


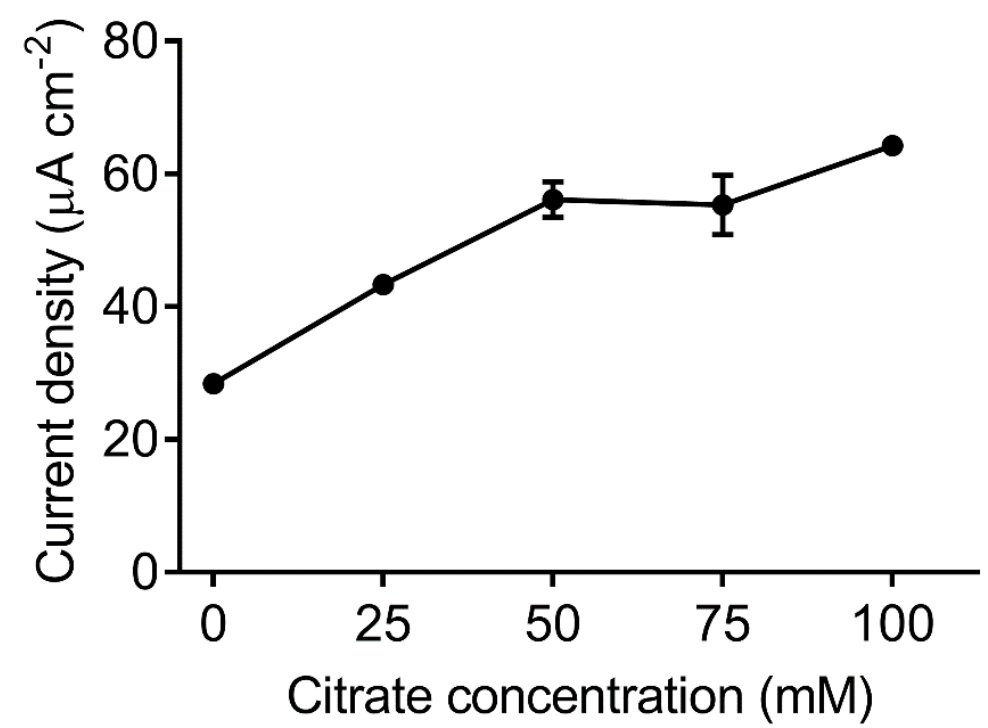

Figure 3.8. Citrate dependence of biosensor \#3. A fixed concentration of $50 \mathrm{mM}$ malate was used in each assays; only citrate was varied. Plotted points are the mean of triplicates, with bars representing standard error, though these are sometimes too small to be seen.

A citrate concentration of $100 \mathrm{mM}$ was used to create biosensor \#3. The calibration curve was only linear from $1-10 \mathrm{mM}$ (Figure 3.9). However, the sensitivity, $3.2 \mu \mathrm{A} \mathrm{mM}^{-1} \mathrm{~cm}^{-2}$, was 30-fold greater than biosensor \#1 and 6-fold greater than biosensor \#2. The baseline noise was also the greatest of the three sensors, with an average reading of $4 \mu \mathrm{A} \mathrm{mM}^{-1} \mathrm{~cm}^{-2}$ and a standard deviation of $0.19 \mu \mathrm{A} \mathrm{mM}^{-1} \mathrm{~cm}^{-2}$. The estimated LOD was also the best of the three sensors, at $0.18 \mathrm{mM}$. 


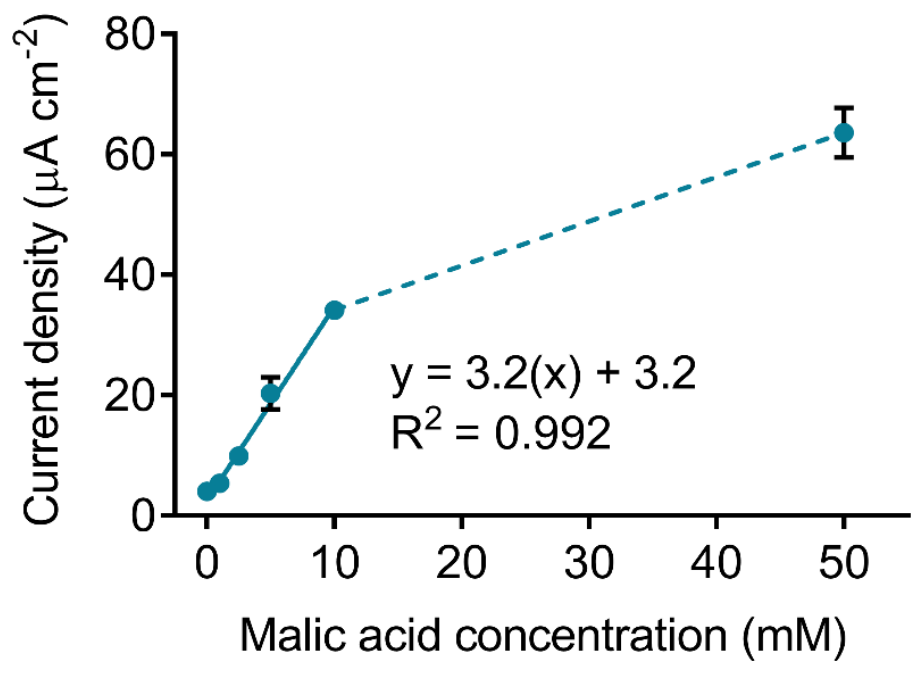

Figure 3.9. Calibration curve for biosensor \#3. The line of best fit (and associated $R^{2}$ ) is for the range $1-10$ $\mathrm{mM}$ malic acid Plotted points are the mean of triplicates. Bars represent standard error, though are oftentimes too small to be seen.

A side-by-side comparison of the three biosensors is shown in Table 3.2.

Table 3.2. Key operational parameters of the three biosensors constructed in this chapter.

\section{Biosensor \#1}

$\left(\mathrm{Mg}^{2+}\right)$

$50-200$

0.11

3.3

125
Biosensor \#2

$\left(\mathrm{Mn}^{2+}\right)$
Biosensor \#3

$\left(\mathrm{Mn}^{2+}+\right.$ citrate $)$
Linear range $(\mathrm{mM})$

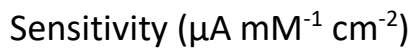

Limit of detection (mM)

Response time (s)
$1-10$

0.18

0.85 


\subsubsection{Spectrophotometric assays using $\mathrm{Mn}^{2+}$ and citrate}

To investigate whether the increased sensitivity caused by $\mathrm{Mn}^{2+}$ and citrate was the result of modified enzyme behaviour, the spectrophotometric assays described in section 2.2.2.4 were repeated in the presence of these two additives. As shown Figure 3.10, these new MichaelisMenten plots were compared to that obtained when using $200 \mathrm{mM} \mathrm{Mg}^{2+}$ in Chapter 2 (Figure 2.15).

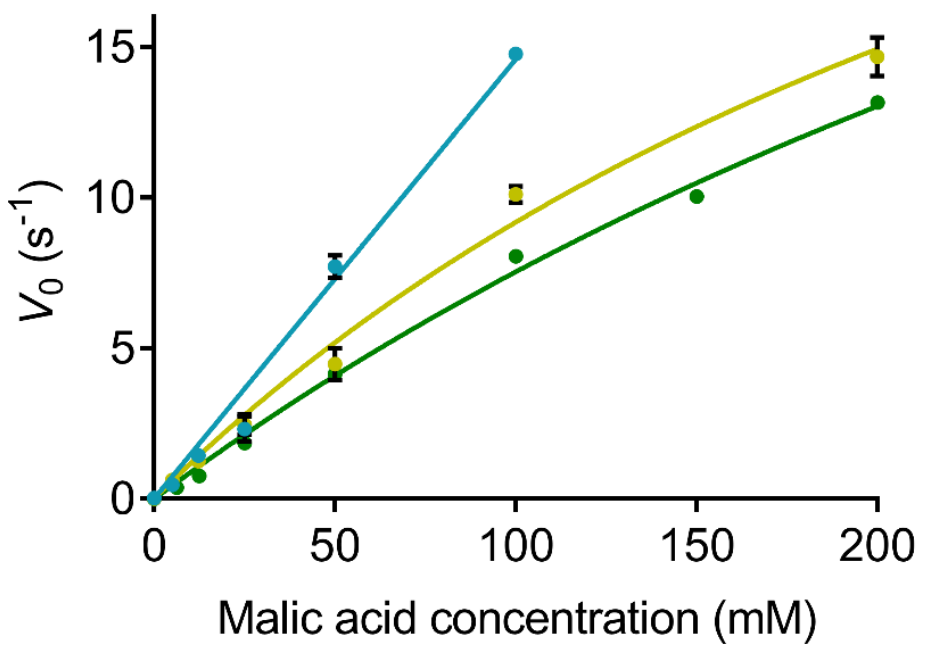

Figure 3.10. Comparison of spectrophotometric assays using $200 \mathrm{mM} \mathrm{Mg}^{2+}$ (green), $200 \mathrm{mM} \mathrm{Mn}^{2+}$ (yellow) and $200 \mathrm{mM} \mathrm{Mn}{ }^{2+}+100 \mathrm{mM}$ citrate (blue). The change in initial velocity $\left(V_{0}\right)$ has been plotted against increasing concentrations of malate. Each set of assays used $300 \mathrm{mM} \mathrm{NH}_{4}{ }^{+}$and $25 \mathrm{mM} \mathrm{NAD}^{+}$, with the data for $\mathrm{Mg}^{2+}$ based assays being taken from Figure 2.15. Rates were measured through the increase in absorbance at 340 $\mathrm{nm}$, corresponding to the reduction of $\mathrm{NAD}^{+}$. Plotted points were fitted with the Michaelis-Menten equation and are the mean of triplicates, with bars representing standard error, though these are oftentimes too small to be seen.

For each of the plots, saturation kinetics were not observed, making the calculated MichaelisMenten parameters “apparent". Substituting $\mathrm{Mg}^{2+}$ with $\mathrm{Mn}^{2+}$ results in a reduction of the apparent $K_{\mathrm{M}}$ from 540 to $340 \mathrm{mM}$ and a decrease in apparent $k_{\text {cat }}$ from 48 to $40 \mathrm{~s}^{-1}$. Comparing the plots at $100 \mathrm{mM}$ malate, $\mathrm{Mn}^{2+}$-based assays produced an initial velocity 1.3fold larger than $\mathrm{Mn}^{2+}$-based assays did. Michaelis-Menten parameters could not be calculated using the plot for $\mathrm{Mn}^{2+}+$ citrate assays. However, the velocity at $100 \mathrm{mM}$ malate was 1.5 fold greater than that of $\mathrm{Mn}^{2+}$ - based assays. 
The increase in velocity for each combination of additives followed the trend seen for the linear responses of electrode-based assays: $\mathrm{Mg}^{2+}<\mathrm{Mn}^{2+}<\mathrm{Mn}^{2+}+$ citrate. This suggests that the linear range of each biosensor is directly related to the apparent $K_{\mathrm{M}}$ of AsME(R181Q).

\subsubsection{Testing other activating compounds described in literature}

L-aspartate, succinate and fumarate have been reported to increase the activities of the malic enzymes E. coli, human, and cauliflower (Brassica oleracea), respectively (Bologna et al., 2007, Grissom et al., 1983, Su et al., 2009). Therefore, I tested them for their ability to amplify the signal of biosensors \#1 and \#2. Compounds were tested at concentration ranges 1- $10 \mathrm{mM}$ (aspartate), 5-50 mM (succinate), and $0.2-10 \mathrm{mM}$ (fumarate). In each case, no increase in the current response was observed.

\subsection{Discussion}

\subsubsection{The electrode}

Many of the malate biosensors reviewed in chapter one have successfully employed gold working electrodes. Among them are some of the most sensitive devices (Giménez-Gómez et al., 2017, Monošík et al., 2012b), indicating that gold is capable of partaking in rapid electron-transfer kinetics, while also being able to interface with a wide range of electron mediators, allowing for flexible sensor architectures. The device in this study was developed using gold screen-printed electrodes - electrode systems which are printed as metallic inks onto a ceramic or plastic material. These were chosen over standard rod electrodes due to their miniature size, high reproducibility, and single-use design that mitigates the need for the electrode polishing used to obtain uniform surfaces (Renedo et al., 2007). The electrodes employed in this study were also relatively cost-effective, at approximately NZ $\$ 8$ each. These aspects increase the ease-of-use of the device and are important considerations for downstream translation to end users. However, there are some drawbacks. In particular, I showed that the promising electron mediator, hexacyanoferrate, which is frequently used in malate biosensors, was not useable due to its magnesium-dependent electrode fouling (and switching the metal ion to $\mathrm{Mn}^{2+}$ produced a brown precipitate). 


\subsubsection{Selection of the mediator}

Each of the candidate electron mediators have been used previously for the electrocatalytic oxidation of NADH. To an extent, the selection procedure used in this study, as well as the candidate choices, were modelled on the methods described by Giménez-Gómez et al. (2017).

The peak oxidation potentials determined in this study correspond well to those described by Giménez-Gómez et al. (2017). However, a key difference is that my study prioritised mediators that produced the greatest signal generation over those with the lowest oxidation potential. This was done in anticipation that a sensor with an ultra-wide linear range may struggle to resolve small differences in malic acid concentration, which could be potentially remediated by increasing the sensitivity through the use an efficient electron mediator.

Giménez-Gómez et al. (2017) used the maximum current densities from cyclic voltammograms to influence their selection of the mediator. However, the data in this study showed that this method is not necessarily the best for obtaining the largest signal. For example, the oxidation peak current when using gallocyanine corresponds to $410 \mu \mathrm{AmM}^{-1}$ $\mathrm{cm}^{-2}$, which is twice as large as the peak seen when using DCPIP, $200 \mu \mathrm{A} \mathrm{mM}^{-1} \mathrm{~cm}^{-2}$. However, in the presence of NADH, DCPIP generated a signal four times larger than gallocyanine (Table 3.1), indicating that the former is a better chemical oxidant of the cofactor in solution. A similar study has been published (Prieto-Simón and Fàbregas, 2004), which also compares the signal generated by various mediators in the presence of NADH, albeit using differing environmental conditions. DCPIP was likewise shown to produce the largest current compared to other commonly used mediators such as hexacyanoferrate and Meldola's blue.

A wide range of mediators have been successfully employed in published malate biosensors, many of which were not included in my screen. Due to the diversity of architectures, it is difficult to discern whether other mediators would be better suited for the device described here. The comparison of mediator performance between biosensors is made more complex by certain studies using diaphorase, an enzyme that catalyses the electron transfer between $\mathrm{NAD}(\mathrm{P}) \mathrm{H}$ and mediators, as well as only few studies testing multiple mediators before selecting one. One such study, by Bucur et al. (2006), does measure the analytical signal generated when using a variety of different mediators, with DCPIP again producing the 
largest current. However, the comparison is limited, as their biosensor uses a malate:quinone oxidoreductase, which does not produce $\mathrm{NAD}(\mathrm{P}) \mathrm{H}$, instead reducing mediators directly via the integrated flavin group.

Improved sensitivity may be gained by using a more soluble mediator: the trend shown in Figure 3.5 indicates that the optimal concentration of DCPIP has not been reached at $0.5 \mathrm{mM}$, though this was maximum able to be tested due to the solubility limits of the stock solution.

\subsubsection{Development of the sensor architecture}

The stock solutions used in assays were all close to the solubility limits of each compound, leaving little space for further optimisation by increasing the concentration of reagents. Two components that were not specifically optimised were the enzyme concentration and the time allowed for the enzymatic reaction to proceed once the malic acid sample had been added. The biosensor was built with a working concentration of 10.2 $\mu \mathrm{M}$ AsME(R181Q) and a twominute reaction time, as the corresponding spectrophotometric activity assays using this concentration of enzyme showed linear rates up to this time. Reducing the incubation time was avoided to reduce the influence of a short $(<10 \mathrm{~s})$ pre-steady-state phase at the start of assays, where rates were not linear and slower than the steady-state kinetics.

The total volume of each assay was $50 \mu \mathrm{L}$, with $30 \mu \mathrm{L}$ consisting of the necessary assay reagents, leaving $20 \mu \mathrm{L}$ for the sample containing malic acid. The need for this dilution could be completely removed, thus making the device even easier to use, by making the sensor 'reagentless', i.e. by immobilising all the reagents needed onto the electrode itself, so that users of the device need only add the sample to begin measurement. Attempts have been made to create reagentless malic acid biosensors using MDH-based systems; however, while methods of enzyme immobilisation are well-established, co-immobilising the other assay components can be problematic. Giménez-Gómez et al. (2017) attempted to entrap the MDH enzyme, $\mathrm{NAD}^{+}$and electron mediator in a polypyrrole film, but reported issues of leaching and incompatibility of certain mediators with polypyrrole, which acted to decrease the storage stability of the sensor. Making the sensors in this current study reagentless may be particularly challenging, as five or six different compounds would have to be entrapped, depending on the sensor. A potential alternative route is to combine reagents in their powdered form with the lyophilised enzyme to create at tablet, which is then dissolved in a 
specific volume of testing solution before the mixture is applied to the biosensor for the measurement. This method has been used for several spectrophotometric commercial testing kits, such as the UniTab range from Unitech (https://www.unitechscientific.com/), which includes a kit for malic acid, though this approach is yet to be used with amperometric sensors.

\subsubsection{Biosensor performance}

A novel feature of my biosensor is its tuneable linear range. The three calibration plots have been overlaid in Figure 3.11.

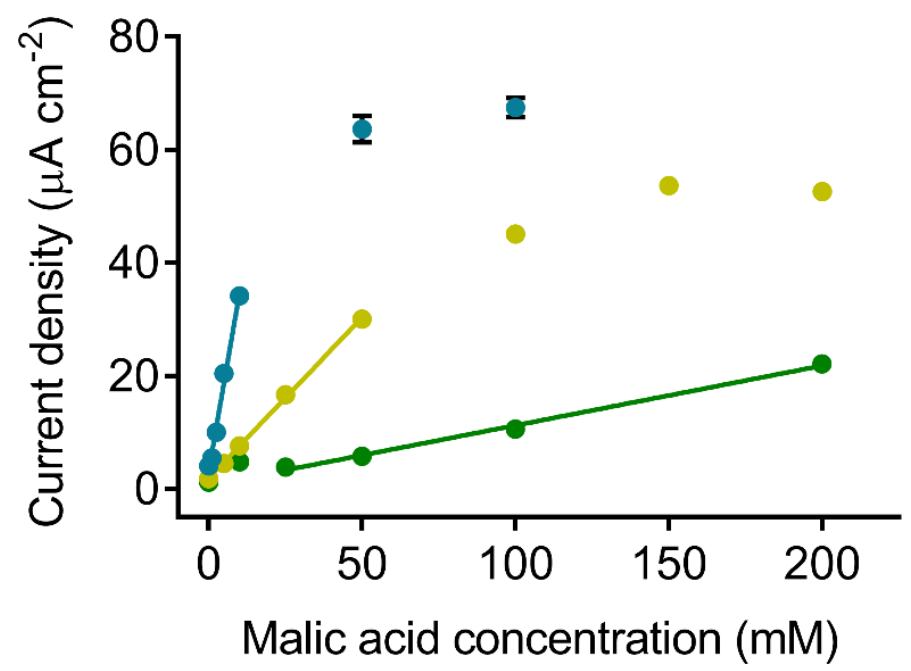

Figure 3.11. Superimposed calibration curves for biosensor \#1 (green; using $\mathrm{Mg}^{2+}$ ), biosensor \#2 (yellow; $\mathrm{Mn}^{2+}$ ) and \#3 (blue; $\mathrm{Mn}^{2+}$ plus citrate). Data taken from Figures 3.6, 3.7 and 3.9.

No such sensor has been described in the academic field of malate sensing, nor to the best of my knowledge, in the broader field of enzyme-based biosensors. This mechanism does however share certain similarities to a concept described for DNA-based sensors, where multiple receptors with different affinities for the target analyte, a specific nucleic acid sequence, have been used together to extend or narrow the linear range of the sensor (ValléeBélisle et al., 2012). 
An increase in sensitivity is seen from biosensor \#1 to biosensor \#2 when substituting the divalent metal ion from $\mathrm{Mg}^{2+}$ to $\mathrm{Mn}^{2+}$ : using $\mathrm{Mn}^{2+}$ in assays decreased the $K_{\mathrm{M}}$, which resulted in greater amounts of $\mathrm{NAD}^{+}$being produced at a given concentration of malate. Changing the divalent metal ion has been reported to alter the kinetics of multiple different enzyme classes, including malic enzymes (Jernejc and Legiša, 2002, Knape et al., 2017). However, a direct comparison of the kinetics that use $\mathrm{Mg}^{2+}$ and $\mathrm{Mn}^{2+}$ has not been performed for AsME or the R181Q mutant. In AsME(R181Q), the divalent metal ion helps co-ordinate malate within the active site (Karsten and Cook, 2007), though it is difficult to predict the precise mechanism of how $\mathrm{Mn}^{2+}$ decreases the $K_{\mathrm{M}}$. Investigations into how altering the divalent cation effects enzyme kinetics have been conducted for DNA polymerase, with the proposed mechanisms being complex: the atomic radii of the metal ion, its coordination geometry and its ability to change $\mathrm{p} K_{\mathrm{a}}$ of nearby molecules are all thought to be factors that change enzyme activity (Vashishtha and Konigsberg, 2016).

The action of citrate remains unclear. The nature of an enzyme activator can be deduced by the changes to $K_{\mathrm{M}}$ and $V_{\max }$ (Baynes and Dominiczak, 2009), though these could not be determined using spectrophotometric assays due to saturation kinetics not being observed. Previous work has shown citrate to be an inhibitor of the malic enzyme from Yarrowia lipolytica, though the mode of inhibition was not investigated (Zhang et al., 2013). Malic enzyme has been linked to the citric acid cycle. It is possible that citrate acts as an allosteric activator, as other molecules from citric acid cycle have been shown to be allosteric regulators of malic enzymes from a range organisms. These include fumarate and succinate, which function as allosteric activators of the human mitochondrial NAD(P)-malic enzyme (Su et al., 2009), glucose-6-phosphate as an allosteric activator of the E. coli NADP-malic enzyme (Bologna et al., 2007), and acetyl-CoA as an allosteric inhibitor of the malic enzyme from the bacterium Rhodopseudomonas palustris (Sato et al., 2010).

Overall, the three sensors described in this chapter have characteristics that compare favourably to others described in the literature (as reviewed in Chapter 1 and Matthews et al., 2021). The linear range, sensitivity and LOD of each biosensor have been compared to those in the literature in Figures 3.12, 3.13 and 3.14. 


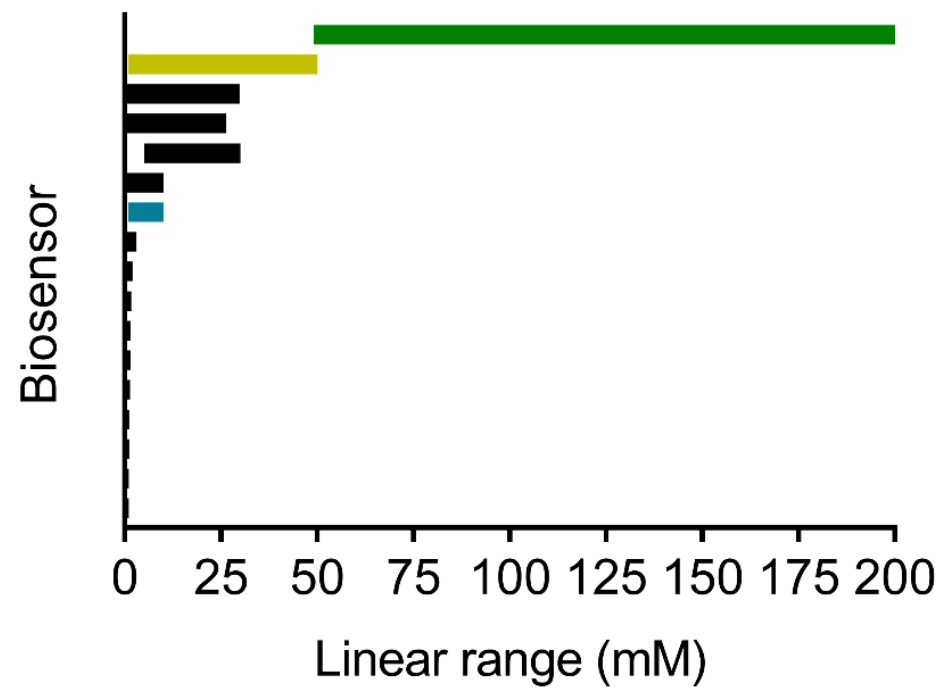

Figure 3.12. Comparison of the linear ranges of biosensors \#1 (green), \#2 (yellow) and \#3 (blue) with those in the literature (black). The linear ranges of 14 of the published malic acid biosensors are shown. The ranges of 21 other sensors, whose upper limits are less than $1 \mathrm{mM}$, have been omitted due to being too small to be visualised.

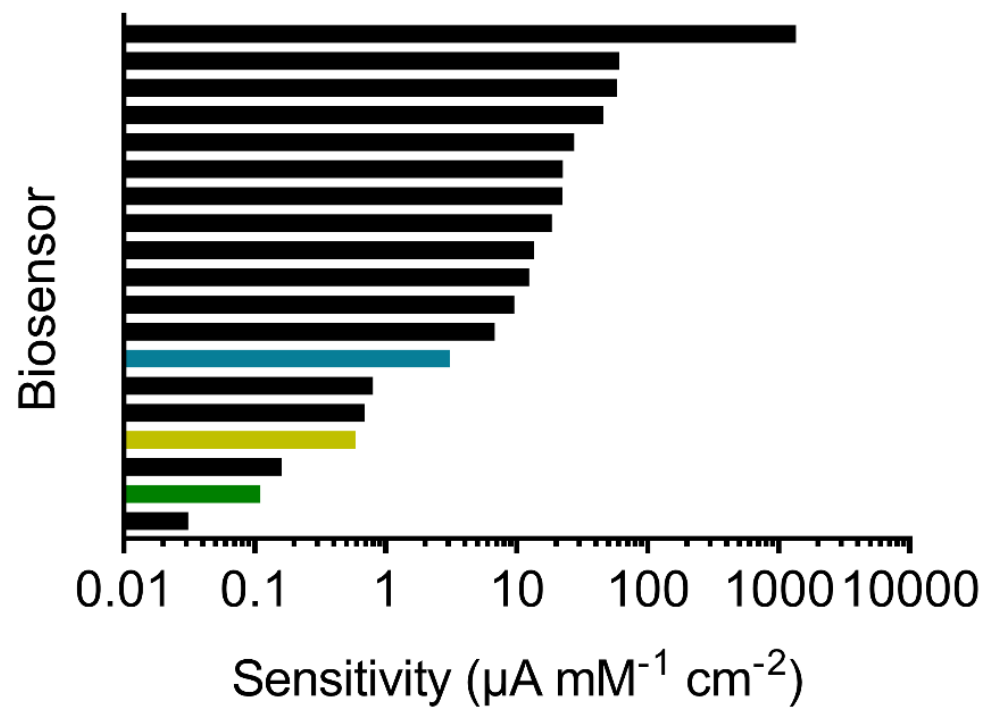

Figure 3.13. Comparison of the sensitivities of biosensors \#1 (green), \#2 (yellow) and \#3 (blue) with those in the literature (black). Only sensitivities that were reported, or could be calculated, as current density (i.e. normalised for electrode area) are included. Note that sensitivity is plotted on a logarithmic scale. 


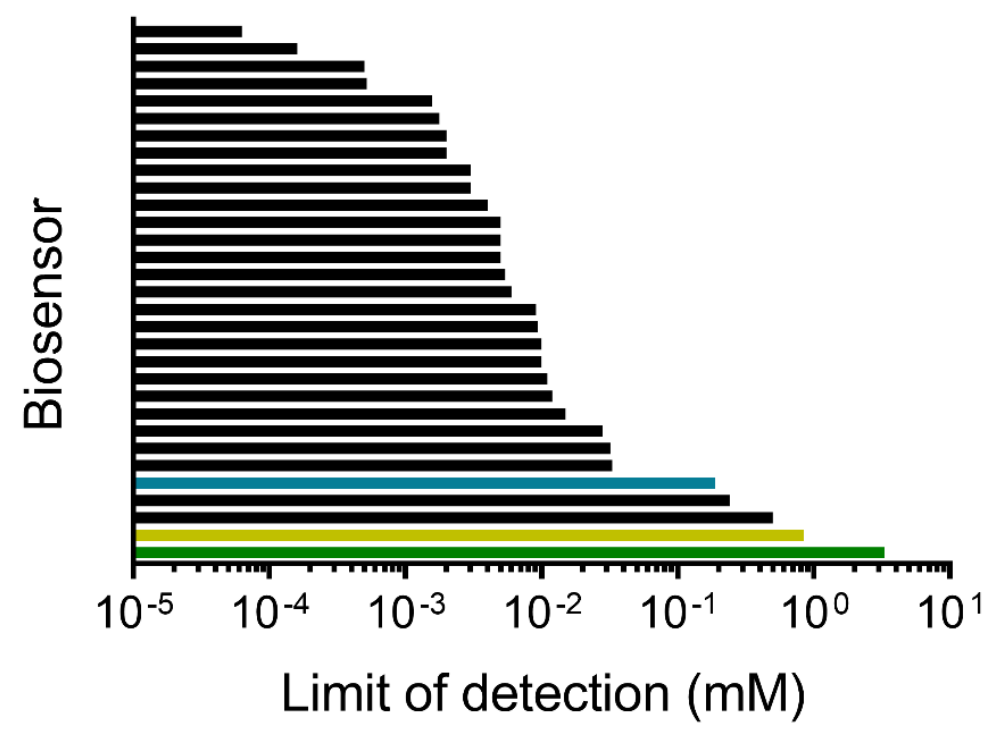

Figure 3.14. Comparison of the limit of detection for biosensors \#1 (green), \#2 (yellow) and \#3 (blue) with those in the literature (black). Note that the limit of detection is plotted on a logarithmic scale.

Biosensor \#1 has the greatest linear range $(50-200 \mathrm{mM})$ of any amperometric malate sensor described to date. The ability to monitor malate concentrations over a span of $150 \mathrm{mM}$ is over 5-fold higher than the device created by Röhlen et al. (2017), which previously held the widest linear range (top black bar in Figure 3.12). Indeed, even biosensor \#2, with its reduced linear range of 1 - $50 \mathrm{mM}$ malate, is still superior to the Röhlen et al. device.

Conversely, biosensor \#1 is amongst the sensors with the lowest sensitivity and has the highest LOD, making it one of the worst at resolving small differences in malic acid concentration. However, biosensor \#1 remains useful, despite the low ranking of these two operational parameters, as the sensor is well-suited to monitor the large changes in malic acid that occur during the growth and maturation phases of grapes on the vine. The LOD is appropriate for measuring the $>5 \mathrm{mM}$ per day change in malic acid that can occur during its accumulation and degradation as the grape grows and matures (Volschenk et al., 2006). Moreover, the upper limit of the linear range, $200 \mathrm{mM}$, corresponds to the maximum malate concentration found in wine grapes (Volschenk et al., 2006), suggesting that it will be possible for viticulturists to test their samples without having to dilute them.

The linear ranges of the two more sensitive devices, biosensors \# 2 and \# 3, are better suited for monitoring post-harvest winemaking processes, such as the degradation of malic acid by fermenting yeast or its removal during malolactic fermentation. The lower limit of the linear range for biosensor \#3, $1 \mathrm{mM}$, is close to the effective endpoint of malolactic fermentation 
(0.75 mM; https://www.awri.com.au/), and coupled with the low LOD, would be useful for fine-tuning the malic acid concentration towards the end of the winemaking process.

Given my focus on developing biosensors with parameters that make them suitable for use by viticulturists and winemakers, the next chapter explores the performance of the three devices in a range of "real world" scenarios. 


\section{Chapter Four - Applying real samples to the device}

\subsection{Chapter introduction}

In previous chapters, the biosensor was developed using tightly controlled conditions and pure solutions of reagents. Conversely, real wine and grape juice samples are highly complex, containing hundreds of different compounds, some of which may adversely affect readings (Sáenz-Navajas et al., 2015, López et al., 2002, Gil et al., 1996).

In grapes, the most abundant compounds are sugars, predominantly glucose and fructose. These are followed by organic acids, phenolics, nitrogenous compounds and minerals. An indication of the relative concentrations of these compounds in wine and grape juice is given in Table 4.1, though it should be noted that in winemaking the concentrations of each of these are highly variable, being dependent on a wide range of factors that include: the grape cultivar used and its maturity, environmental conditions, and the stage and nature of the winemaking process itself (Eyduran et al., 2015, Ough, 1964, Volschenk et al., 2006). 
Table 4.1. Relative quantities of the main constituents of wine. Data reproduced with permission from the NZ Institute of Chemistry (https://nzic.org.nz/). The table has been modified to show the mM concentrations of each compound, calculated where possible.

\begin{tabular}{lcc} 
Compound & Grape juices & Wines \\
\hline Water & $79 \%(\mathrm{w} / \mathrm{w})$ & $85 \%(\mathrm{w} / \mathrm{w})$ \\
Sugars & & \\
Fructose & $610 \mathrm{mM}$ & $4 \mathrm{mM}$ \\
Glucose & $550 \mathrm{mM}$ & $3 \mathrm{mM}$ \\
Alcohols & & \\
Ethanol & Trace & $12.5 \%(\mathrm{v} / \mathrm{v})$ \\
Glycerol & 0 & $65-110 \mathrm{mM}$ \\
Organic acids & & \\
Tartaric & $40-80 \mathrm{mM}$ & $30-70 \mathrm{mM}$ \\
Malic & $15-200 \mathrm{mM}$ a & $0-45 \mathrm{mM}$ \\
Citric & $1 \mathrm{mM}$ & $1 \mathrm{mM}$ \\
Lactic & 0 & $3-40 \mathrm{mM}$ \\
Succinic & 0 & $8 \mathrm{mM}$ \\
Ascorbic & $75 \mu \mathrm{M}$ & $\mathrm{Trace}$ \\
Phenols & - & $0.1-0.25 \mathrm{~g} / \mathrm{L}$ \\
Nitrogenous compounds & & \\
Ammonium & $3 \mathrm{mM}$ & $17 \mathrm{mM}$ \\
Amino acids & $0.04 \%(\mathrm{w} / \mathrm{w})$ & $0.1-0.25 \%(\mathrm{w} / \mathrm{w})$ \\
Minerals & & \\
Calcium & $4 \mathrm{mM}$ & $1-3 \mathrm{mM}$ \\
Chloride & $3 \mathrm{mM}$ & $1-6 \mathrm{mM}$ \\
Magnesium & $6 \mathrm{mM}$ & $1-5 \mathrm{mM}$ \\
Phosphate & $3 \mathrm{mM}$ & $0.25-9 \mathrm{mM}$ \\
Potassium & $50 \mathrm{mM}$ & $15-30 \mathrm{mM}$ \\
Sodium & Trace & $2 \mathrm{mM}$ \\
Sulfate & $2 \mathrm{mM}$ & $7-30 \mathrm{mM}$ \\
\hline & & \\
\hline & & \\
& & \\
\hline
\end{tabular}

a The concentration of malic acid has been modified from the original data to include the concentrations described by Volshenk, Vuuren and Viljoen-Bloom (2006).

${ }^{b}$ Ascorbic acid concentrations were added to the table using the data given by Ribereau-Gayon et al. (2006).

\subsubsection{Compounds that commonly interfere with malate biosensors ${ }^{5}$}

For a measurement to reflect the actual malate concentration within a sample, a biosensor must be highly selective, and it must be resistant to the effects of interfering compounds. Interference studies have been carried out for approximately half of the enzyme-based amperometric devices reviewed in Tables 1.1-1.3. The findings of these studies are summarised in Table 4.2.

\footnotetext{
5 'Common compounds that interfere with malate biosensors' is a section that appears in my published review article (see section 1.1), though has been modified here to focus on AsME and malic enzymes.
} 
Table 4.2. Interference studies conducted on enzyme-based amperometric malate biosensors, ordered by date of publication.

Compounds tested for interference ${ }^{a}$

Acetic acid, adipic acid, alanine, ascorbic acid, butyric acid, calcium, chloride, citric acid, glutamic acid, glycine, lactic acid, leucine, lysine, magnesium, oxalic acid, oxaloacetic acid, potassium, sodium, tartaric acid

Ethanol, white and red wine matrices

Oxaloacetate, pyruvate

NADH, NADPH

Acetic acid, ascorbic acid, citric acid, L-lactate, succinic acid, sulfite, tartaric acid, white and red wine matrices

Ascorbic acid

Ascorbic acid, citric acid, glutamic acid, tartaric acid

Red wine matrix

White wine matrix

Ascorbic acid

Gallic acid, red wine matrix

Gallic acid, red wine matrix

Acetic acid, arabinose, ascorbic acid, citric acid, ethanol, fructose, galactose, gluconic

acid, glucose, glycerol, D-lactic acid, L-lactic acid, tartaric acid

Acetic acid, L-ascorbic acid, citric acid, ethanol, fructose, glucose, L-lactic acid, red wine matrix, tartaric acid

Acetic acid, L-arabinose, ascorbic acid, citric acid, ethanol, D-fructose, D-galactose,

gluconic acid, D-glucose, glutamine, glycerol, D-lactic acid, L-lactic acid, tartaric acid

Acetic acid, ascorbic acid, citric acid, ethanol, fructose, gluconic acid, glucose,

glycerol, L-lactate, tartaric acid

Ascorbic acid, citric acid, lactic acid, oxalic acid, tartaric acid

${ }^{a}$ Compounds found to interfere with biosensor performance are highlighted in bold typeface.
Ref.

(Prodromidis et al., 1996)

(Messia et al., 1996)

(Gajovic et al., 1997)

(Gajovic et al., 1998)

(Katrlík et al., 1999)

(Maines et al., 2000)

(Arif et al., 2002)

(Esti et al., 2004)

(Lupu et al., 2004)

(Manzoli et al., 2004)

(Bucur et al., 2006)

(Gurban et al., 2006)

(Gamella et al., 2010)

(Monošík et al., 2012a)

(Vargas et al., 2016)

(Giménez-Gómez et al., 2017)

(Mundaca-Uribe et al., 2017)

As with other types of electrochemical biosensors (Rahman et al., 2010, Rathee et al., 2016), ascorbic acid interference pervades the malate biosensor literature. Of the 17 interference studies that have been reported, ascorbic acid was specifically tested in ten and shown to be an interferent in eight of these (Table 4.2). As a strong reducing agent, ascorbic acid is ubiquitous within plants as a tool for mitigating oxidative stress(Asensi-Fabado and MunnéBosch, 2010), with millimolar concentrations found within grapes (Derradji-Benmeziane et al., 2014, Hemraj et al., 2019). The ability of ascorbic acid to reduce the electrode surface, even at low potentials (Borsook and Keighley, 1933), has proven to be a major obstacle in creating devices resistant to generating non-specific current. Most malate sensors have been designed for use in winemaking, where polyphenols present a similar hurdle. These are abundant in red, and to a lesser extent, white wine matrices (Cordova and Sumpio, 2009). Like ascorbic acid, certain polyphenols act as potent reducing agents (Chiorcea-Paquim et al., 2020), while also inhibiting a wide range of enzyme classes (Gil and Rebelo, 2011, NyambeSilavwe et al., 2015, Zolghadri et al., 2019) that includes certain malic enzymes (Pairoba et 
$a l .$, 1996). However, the ability to polyphenols to inhibit AsME, or its mutants, has not yet been investigated.

While compounds such as ascorbic acid and polyphenols interfere by directly reducing the electrode or the mediator, other compounds can interfere with the enzymatic components of malate sensors (Gajovic et al., 1997). Outside of a biosensor context, the influence of various compounds on wild-type AsME activity have been characterised previously (Landsperger and Harris, 1976). Fumarate, at $0.7 \mathrm{mM}$, was the only compound shown have an influence, enhancing activity to $210 \%$ of the control. Many more regulatory compounds have been described for other malic enzymes, which have been collated by Liang et al. (2015). These include compounds found in Table 4.1: citrate, succinate and lactic acid (Su et al., 2009, Zhang et al., 2013), as well as other structurally related compounds from neighbouring parts of the metabolic network, such as oxaloacetic acid. Each of these has been shown to be a source of interference for certain malate biosensors (Table 4.2).

\subsubsection{Measurements in acidic samples}

As described in chapter two, the $\mathrm{pH}$ of grape juice and wine ( $\mathrm{pH} 3-4)$ is too acidic to be added directly to the biosensor without diminishing enzyme activity. In the literature, strategies to deacidify samples are rarely considered, as the high dilution required to lower the malic acid concentration also allows the $\mathrm{pH}$ to be effectively buffered. Only one study, by Maines et al. (2000), created a protective mechanism against $\mathrm{pH}$ inactivation: the enzyme layer was covered with a plastic-based resin, allowing malate concentration to be measured at $\mathrm{pH} 3.3$, though experiments using real samples were not described. The biosensor in this current study has been designed to accommodate the malic acid concentrations found within undiluted grape juice; therefore, a strategy needed to be developed to negate the adverse effects of acidity.

This chapter describes the sensor response when subjected to grape juice and wine. A buffering system was developed to accommodate these acidic samples, and a range of compounds found in wine were tested as potential interfering compounds. 


\subsection{Results}

\subsubsection{Initial grape juice testing}

Initial trials used both Pinot Noir and Pinot Gris grapes sourced from the Hare's Run vineyard in the Wairarapa region of New Zealand. Grapes were picked from the vine in March, corresponding to the late stage of maturation shortly before harvesting (see section 1.2.1). Samples were applied to biosensor \#1, with the readings compared to those of two commercial malic acid testing kits: one supplied by Unitech Scientific and the other from Megazyme.

Using undiluted samples on the sensor failed at first to produce a reading: only large cathodic (negative) currents could be generated. The acidity of the grape juice ( pH 3-4) had overcome the buffering capacity of the $100 \mathrm{mM}$ HEPES used in assays, indicated by a colour change of the mediator DCPIP from blue to pink, a well characterised reaction that occurs at low $\mathrm{pH}$. This was later shown empirically, described in section 4.2.2. Samples were thereafter raised to $\mathrm{pH} 7$ using concentrated $\mathrm{NaOH}$ before being applied to the device, with measured concentrations being corrected for the dilution.

The measurements from the sensor and commercial kits are shown in Figure 4.1. The concentrations determined by each kit were similar, averaging 21 and $24 \mathrm{mM}$ for Pinot Noir and Pinot Gris, respectively. This corresponds well to the expected concentrations of malic acid found in mature grapes prior to harvesting, which is between $7-50 \mathrm{mM}$. The readings from biosensor \#1, however, were much larger: $130 \mathrm{mM}$ for Pinot Noir and $160 \mathrm{mM}$ for Pinot Gris - concentrations that would not be expected in the mature grapes used here, but rather concentrations seen prior to the onset of veraison. The measured concentrations were 6- and 6.5-fold larger compared to the testing kits, respectively.

At this stage in the project, biosensors \#2 and \#3 had not yet been developed. Given that the commercial testing kits suggested that the malate concentrations in the samples were outside the linear range of sensor \#1 (50 - $200 \mathrm{mM})$, juice samples spiked with $100 \mathrm{mM}$ malic acid were testing alongside the original samples. The currents produced now exceeded the linear range, though when extrapolated, corresponded to $250 \mathrm{mM}$ and $320 \mathrm{mM}$. This increase was not consistent with the spiked-in concentration of malate. 


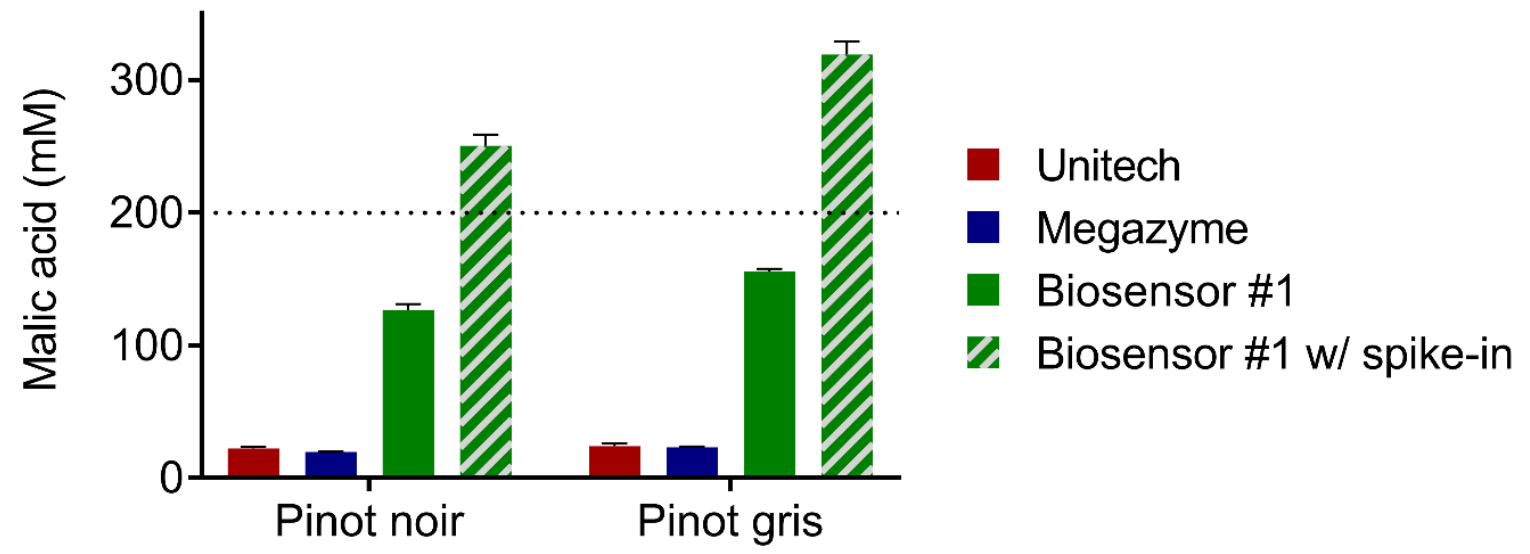

Figure 4.1. Comparison of measurements between biosensor \#1 and commercial test kits. Spiked samples contained an additional $100 \mathrm{mM}$ malic acid. The dotted line represents the upper limit of the linear range for biosensor \#1. Error bars are the standard error of technical triplicates. Grape samples were sourced from the Wairarapa region in New Zealand.

\subsubsection{Development of a buffering system}

Raising the $\mathrm{pH}$ of each sample using $\mathrm{NaOH}$ was time-consuming. As the project continued, the number of real samples available for testing increased, and attempts were made to develop a faster approach. The efforts were focused on the buffer component of sensor assays, with a desired outcome that assay $\mathrm{pH}$ remained unchanged when the real sample was added.

Buffering capacity increases proportionally with the concentration of the buffering molecule (Urbansky and Schock, 2000). Accordingly, initial attempts involved increasing the concentration of HEPES buffer used in sensor assays from $100 \mathrm{mM}$ to $500 \mathrm{mM}$. The change in $\mathrm{pH}$ was measured when the biosensor assay containing this new buffer was mixed with each of 21 different grape juice samples: 10 Pinot Noir and 11 Pinot Gris, sourced from Mt Difficulty Wines (Central Otago, New Zealand). When added, most samples caused a decrease in $\mathrm{pH}$, from the initial $\mathrm{pH}$ of 7.0 to values falling in the range of $\mathrm{pH} 6.76-7.00$. Approximately half of the samples caused the $\mathrm{pH}$ to fall below $\mathrm{pH} 6.8$, which is the lower limit of the effective buffering range of HEPES (pH 6.8 - 8.2) (Good et al., 1966). This meant that the assay mixtures were not effectively buffered and were therefore susceptible to large changes in $\mathrm{pH}$ during the period of the monitored reaction. The initial $\mathrm{pH}$ of the buffer was increased from $\mathrm{pH} 7.0$ to $\mathrm{pH} 7.2$, on the hypothetical basis that even the greatest decrease in 
pH seen, 0.24 units, would lead to assays remaining within HEPES's effective buffering range. The strategy was largely successful, with only one sample causing the $\mathrm{pH}$ to fall below $\mathrm{pH}$ 6.8. However, the range of $\mathrm{pH}$ values after addition of the juice samples actually increased, so that assay mixtures were now between $\mathrm{pH} 6.79$ and $\mathrm{pH}$ 7.20.

The increase in HEPES concentration from $100 \mathrm{mM}$ to $500 \mathrm{mM}$ adversely impacted the sensor's performance, as shown by a diminished response. When using the most sensitive biosensor, \#3 (with $\mathrm{Mn}^{2+}$ and citrate present), $500 \mathrm{mM}$ HEPES pH 7.0 reduced the signal obtained at $10 \mathrm{mM}$ malic acid to $40 \%$ of that produced when using $100 \mathrm{mM}$ HEPES pH 7.0. This attenuation was shown to be $\mathrm{pH}$ dependent, becoming more pronounced as the $\mathrm{pH}$ was increased: using $500 \mathrm{mM}$ HEPES pH 7.2 decreased the signal further, to $30 \%$ of the original. The cause may be linked to oxidation of $\mathrm{Mn}^{2+}$, as indicated by a rapid (within minutes) colour change of the stock solution from light pink to brown. Below $\mathrm{pH} 6.9$, the colour change was much slower, with solutions at pH 6.8 turning brown only after 12 hours. However, even at this lowered $\mathrm{pH}, 500 \mathrm{mM}$ HEPES still reduced the signal to $70 \%$ of the signal obtained when $100 \mathrm{mM}$ HEPES was used instead.

Subsequent attempts focused on creating a buffering system that used a high concentration of the buffering molecule and which could buffer effectively at a lower $\mathrm{pH}$, potentially slowing the oxidation of $\mathrm{Mn}^{2+}$. HEPES was substituted for $500 \mathrm{mM}$ MOPS buffer, $\mathrm{pH}$ 6.7. This buffer has an effective range of $6.5-6.9$, lower than that of HEPES (Good et al., 1966). The buffering capacity appeared to be poorer than HEPES buffer, as shown by all but one sample causing the $\mathrm{pH}$ to fall outside of this effective buffering range (Figure 4.2). However, signal generation was stable and readings were comparable to the original sensor developed with 100 mM HEPES, pH 7.0. 


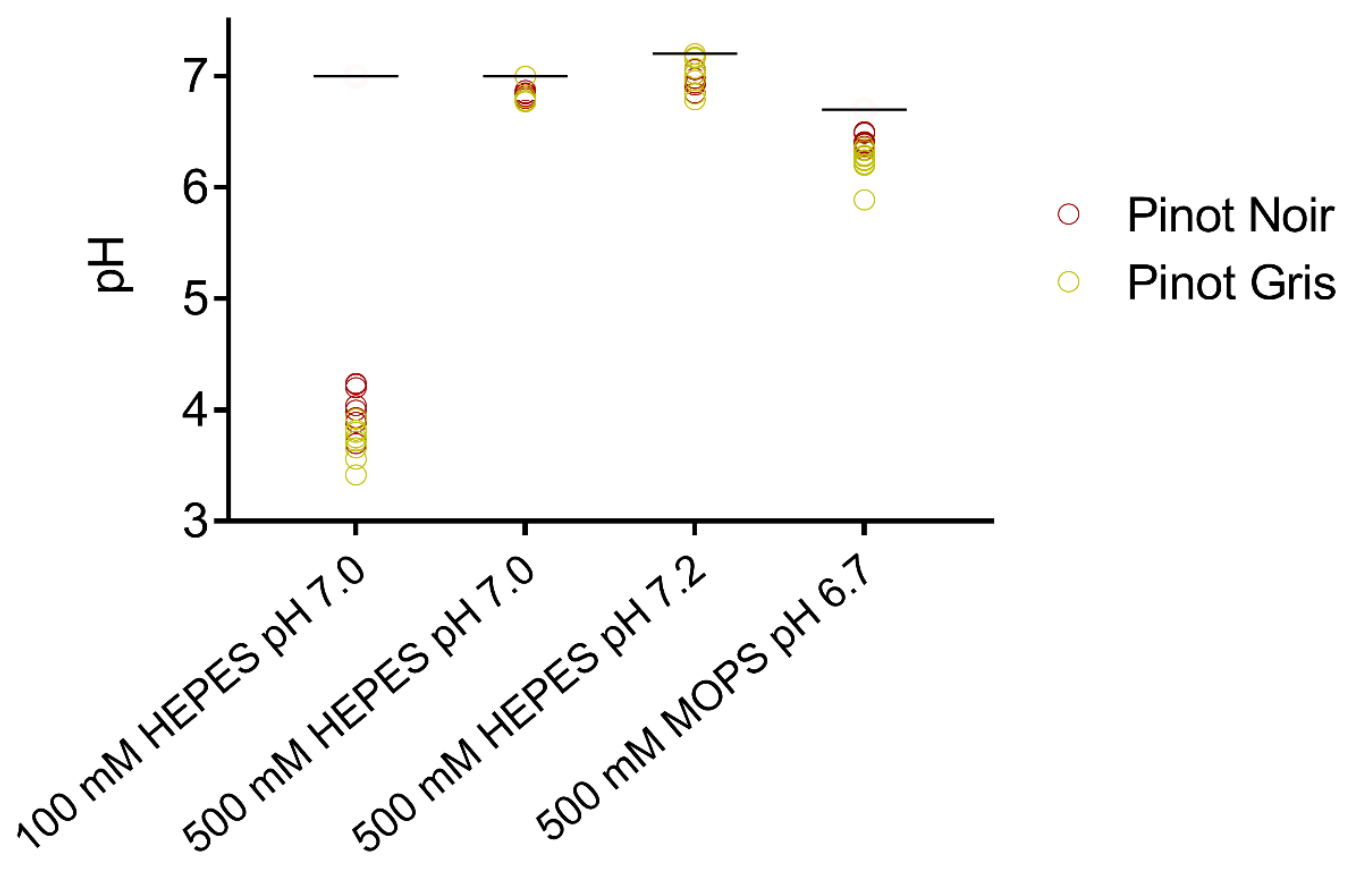

Figure 4.2. $\mathrm{pH}$ variation of sensor assays using various buffers upon addition of real samples. Each solution used the buffering molecule indicated on the graph, along with $200 \mathrm{mM} \mathrm{MnCl}_{2}$ and $300 \mathrm{mM} \mathrm{NH}_{4}{ }^{+}$. Results are shown for 21 grape juice samples (10 Pinot Noir and 11 Pinot Gris). For each juice, a $20 \mu \mathrm{L}$ sample was added to $30 \mu \mathrm{L}$ of assay reagents and then the $\mathrm{pH}$ was measured. Black lines on each plot indicate the original $\mathrm{pH}$ of the buffer before the addition of juice samples.

MOPS was used to investigate the tolerance of biosensor \#3 to small changes in $\mathrm{pH}$. The change in response was measured over the range of $\mathrm{pH} 6-7$, producing a general trend where the response decreased as the $\mathrm{pH}$ increased. However, a stable range was identified between $\mathrm{pH} 6.3-6.7$, where the difference between the highest and lowest signal was less than $6 \%$ (Figure 4.3). 


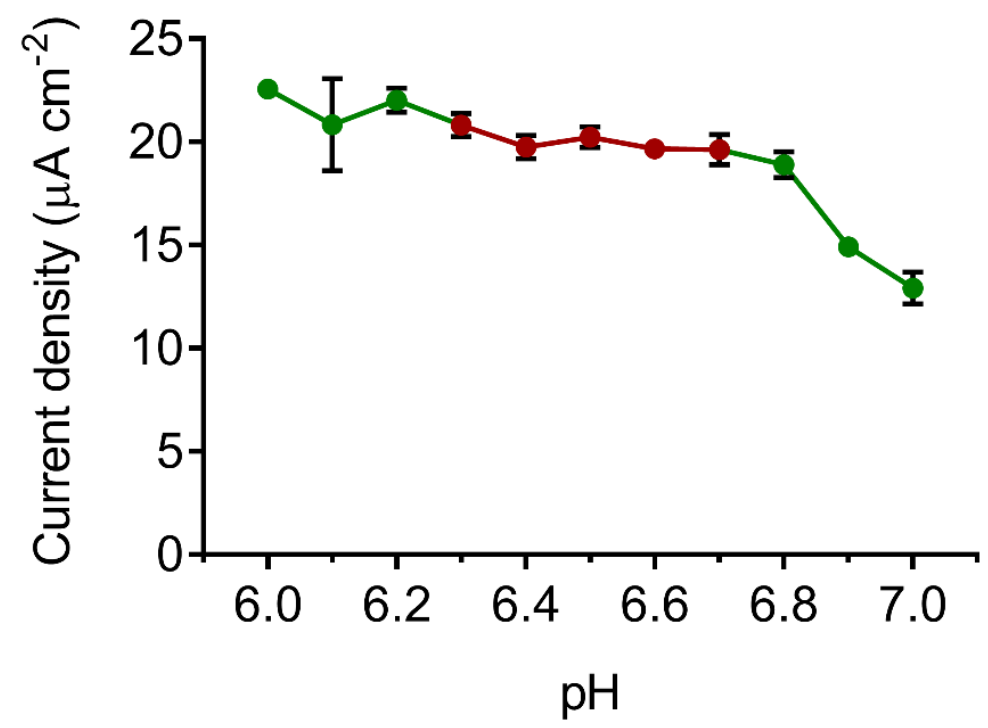

Figure 4.3. The $\mathrm{pH}$ dependence of biosensor \#3 using $500 \mathrm{mM}$ MOPS buffer, $\mathrm{pH}$ 6.7. Assays used $10 \mathrm{mM}$ malate. A region of relative $\mathrm{pH}$ stability, described in the text, is shown in red. Plots are the mean of technical triplicates, with error bars representing standard error, though these are sometimes too small to be seen.

Finally, it was reasoned that MES, with its effective buffering range of 5.5-6.7 (Good et al., 1966) might be best suited for maintaining the $\mathrm{pH}$ within this region of stable activity. Indeed, using an assay mixture with $500 \mathrm{mM}$ MES at pH 6.7, 20 of the 21 juice samples were maintained between $\mathrm{pH} 6.3-6.5$, with one just outside of the stable region at $\mathrm{pH} 6.26$. Sensor assays proved compatible with MES, with comparable current readings to the original calibration curve. Therefore, a new calibration curve was created for biosensor \#3 using 500 $\mathrm{mM}$ MES, $\mathrm{pH}$ 6.7. The MES-based system resulted in a small increase in sensitivity, to 3.95 $\mu \mathrm{A} \mathrm{mM}^{-1} \mathrm{~cm}^{-2}$, which was 1.3-fold greater than that of the original sensor (Figure 4.4). At the same time, a more unstable baseline meant that the limit of detection was approximately twice as high, at $0.32 \mathrm{mM}$. The linear range of biosensor \#3 with MES was also reduced from $0-10 \mathrm{mM}$ malate, to $0-5 \mathrm{mM}$ (Figure 4.4). Nevertheless, the ability to use grape juice samples without dilution or $\mathrm{pH}$ adjustment suggested that this trade-off was worthwhile, to maximise the user-friendliness for winemakers. 


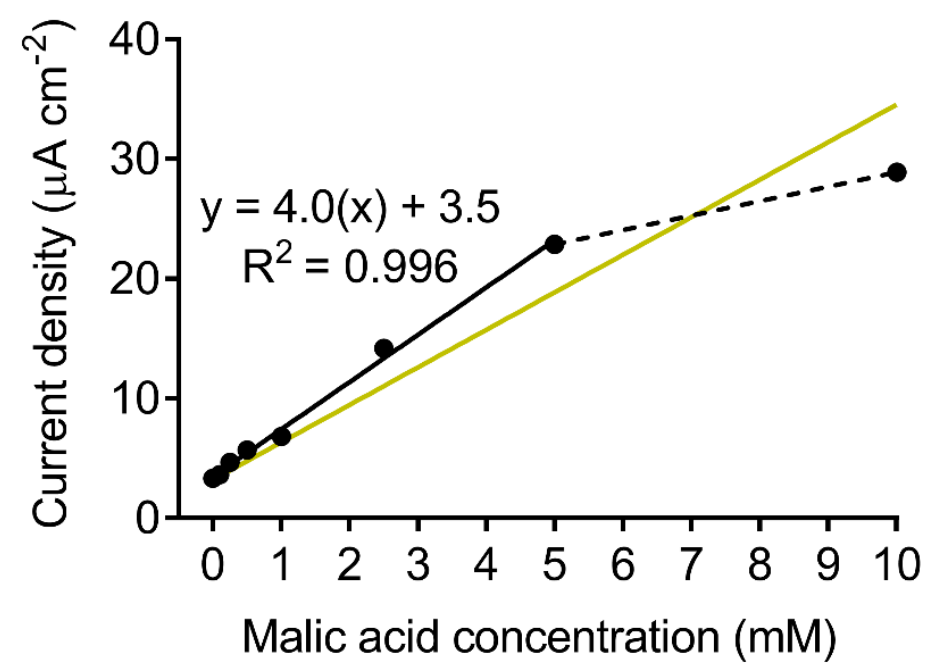

Figure 4.4. Comparison of calibration curves for biosensor \#3 generated using either $500 \mathrm{mM} \mathrm{MES} \mathrm{buffer} \mathrm{pH}$ 6.7 (black) or $100 \mathrm{mM} \mathrm{HEPES} \mathrm{buffer} \mathrm{pH} 7$ (yellow). Points are the mean of triplicate repeats with error bars representing standard error, though these are too small to be seen. Measurements were also performed using 50 and $100 \mathrm{mM}$ malic acid, though these are not shown to make the comparison between linear ranges more clear.

\subsubsection{Measuring malic acid across alcoholic fermentation}

Two time courses, comprising 13 Pinot Noir and 12 Pinot Gris juice samples, were obtained from Mt Difficulty Wines. Each sample had been taken at a different day during the alcoholic fermentation over a three-week period. The malic acid concentrations of these samples were measured with the two commercial test kits and with biosensor \#3, using the MES-based system described in the previous section. The objective was to investigate whether these new samples produced the same level of interference as in the initial assays in section 4.2.1, and if so, to identify any trends in the interference across the fermentation process.

A comparison of the measurements made using the biosensor and the test kits is shown in Figure 4.5. The kits produce concordant measurements, with reported malic acid concentrations between $2-5 \mathrm{mM}$ across samples. These concentrations are within the linear range of the MES-based calibration curve in Figure 4.4. However, unexpectedly high signals were once again produced using the biosensor, far exceeding the upper limits of the linear range. The degree of this unwanted extra signal was not consistent, with current 
measurements being between 2.5 and 4.5 -fold greater than expected based on the commercial kit results. There was also no obvious trend in the level of interference between the early and late stages of the fermentation process, nor did the type of grape have a noticeable effect. 


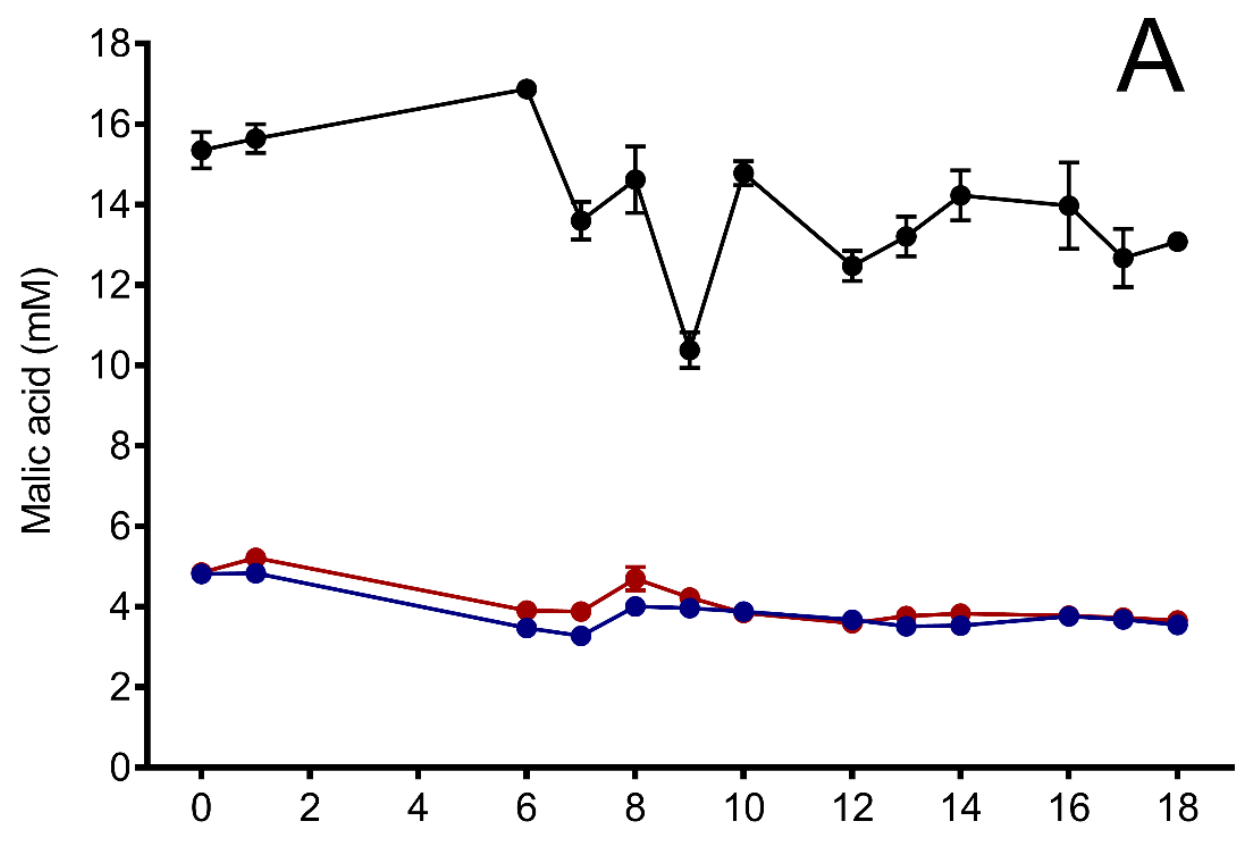

Days during fermentation

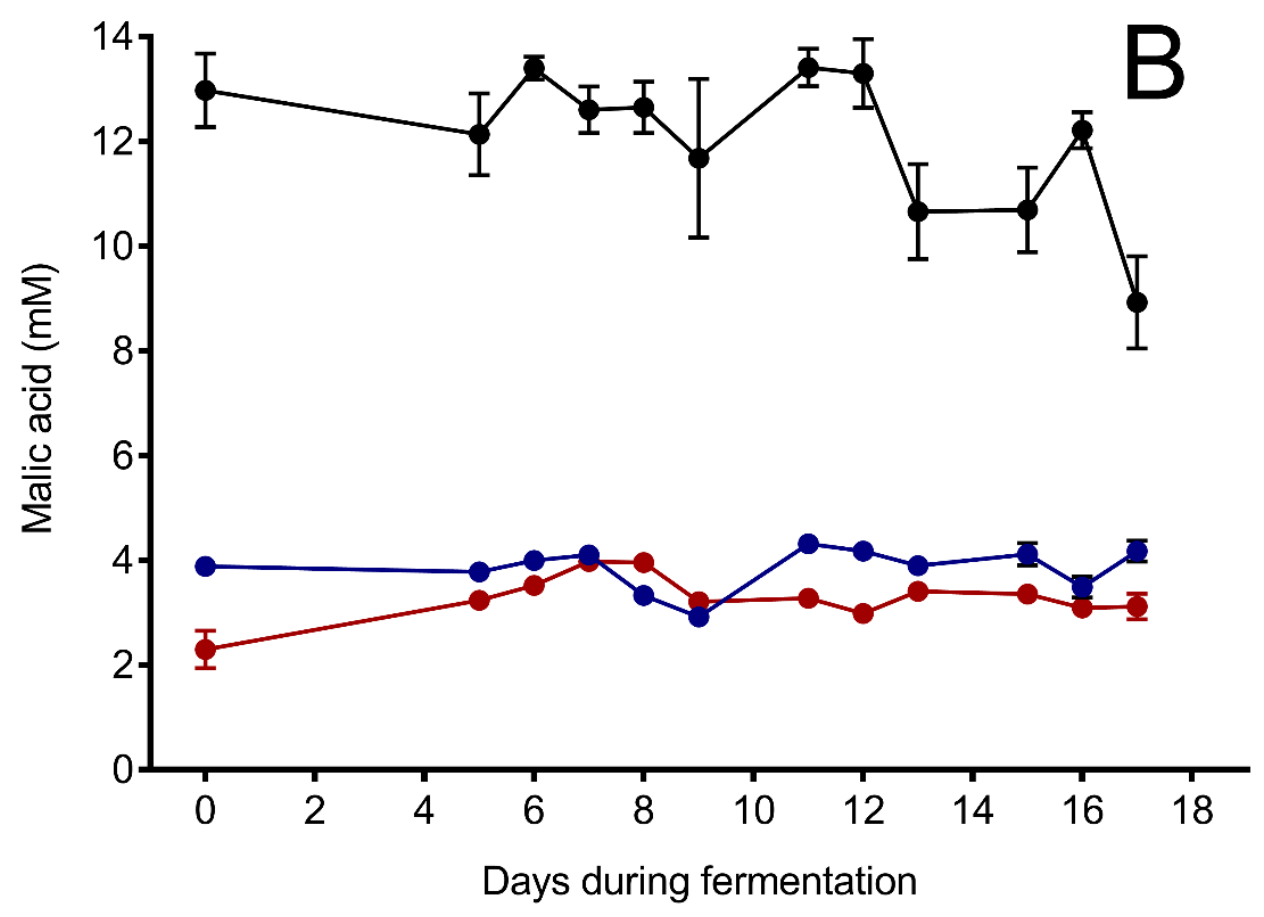

Figure 4.5. Comparison of measurements between the biosensor and commercial kits using grape juice/wine samples from alcoholic fermentation. Black: biosensor \#3 (MES-buffered). Blue: Unitech kit. Red: Megazyme kit. A) Using Pinot Noir grapes. B). Using Pinot Gris grapes. Current readings obtained with biosensor \#3 far exceeded the linear range and are therefore highly unreliable. To show the relative size of the signal, concentrations reported for the sensor were calculated by extrapolating the linear portion of the calibration curve. Error bars represent the standard error of technical triplicates, though are sometimes too small to see. 
A series of deductive experiments was performed to identify which components of the biosensor architecture were susceptible to interference (Figure 4.6). Arbitrarily, the Pinot Noir sample from the ninth day of the fermentation was used for these tests.

The sample, when applied to biosensor \#3, produced a current reading of $31 \mu \mathrm{A} \mathrm{cm}^{-2}$, when only $9.9 \mu \mathrm{A} \mathrm{cm}^{-2}$ was expected. First, to test whether the grape juice matrix was capable of reducing the electrode itself, assays were performed in the absence of AsME(R181Q) or the mediator (DCPIP). Only a very small anodic current was observed, though this was approximately $1 \mu \mathrm{A} \mathrm{mM}^{-1} \mathrm{~cm}^{-2}$ less than the background current obtained in control readings without sample - likely due to the absence of a background reading caused by the presence of the mediator.

Next, the assay was repeated, omitting only the enzyme. After being corrected for the baseline reading, the resulting current was $7.5 \mu \mathrm{A} \mathrm{cm}^{-2}$ larger than expected (Figure 4.6), indicating that the juice matrix was reducing $\mathrm{NAD}^{+}$and/or the mediator. In future sections, this unwanted signal is termed "NAD"/mediator interference", though, having not tested each individually, it is unclear which component is being reduced.

However, this current does not account for the total level of interference seen in the standard assay using the grape juice sample, which corresponds to an extra $14 \mu \mathrm{A} \mathrm{mM}^{-1} \mathrm{~cm}^{-2}$ in addition to the signal generated from direct reduction of the mediator and/or NAD ${ }^{+}$. This shows that the juice matrix interferes with the activity of AsME(R181Q). 

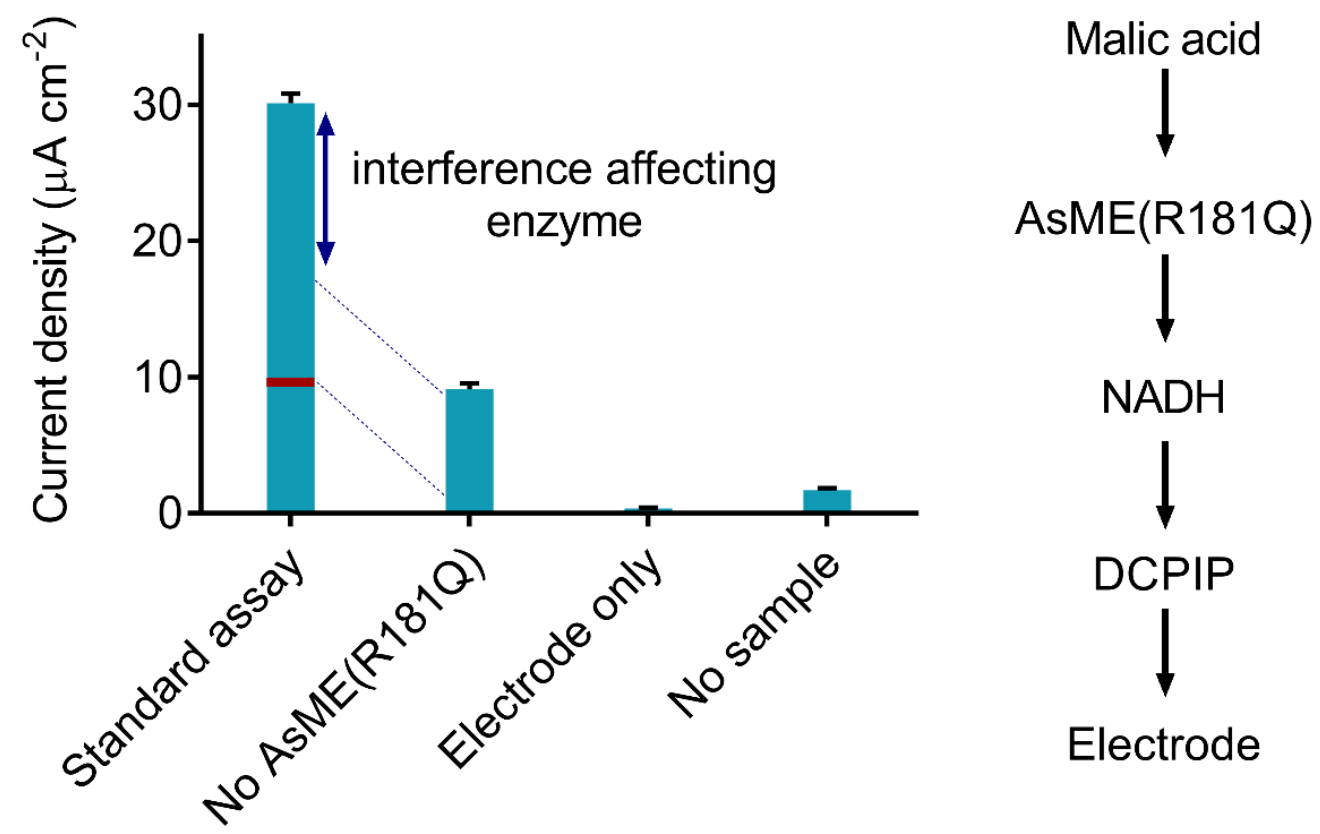

Figure 4.6. Control experiments used to identify the architecture components prone to interference. Assays used biosensor \#3 (MES-buffered) and a Pinot Noir grape juice sample nine days along the fermentation process from Mount Difficulty Wines. A red line has been drawn to show the expected signal of the standard assay, as determined by the average reading of commercial testing kits. Dashed lines are used to help visualise the contribution of the interference signal observed in the absence of enzyme to the total signal of the standard assay. "Electrode only" assays were performed in the absence of AsME(R181Q), NADH or DCPIP. Error bars represent the standard error of technical triplicates. For reference, a schematic for the electrontransfer reactions used by the biosensor has been included.

\subsubsection{Testing specific compounds found in wine for interference}

\subsubsection{Major organic compounds}

In an attempt to identify the specific molecule(s) within grape juice causing the interference, assays were performed with biosensor \#1 in the presence of the major organic compounds found in wine. These included glucose, glycerol, all the organic acids shown in Table 4.1, as well as three unlisted acids: fumaric, acetic and pyruvic acids.

Compounds were tested at a concentration of $100 \mathrm{mM}$, with succinate, fumarate and citrate producing increased signals: $119 \%, 148 \%$ and $151 \%$ of the original signal, respectively 
(Figure 4.7). It was this result with citrate, showing the greatest increase in signal, that led to its use in creating biosensor \#3 (section 3.2.5).

Many of the compounds tested are structurally similar to malic acid and could potentially be used by the enzyme as a substrate. Accordingly, a series of control assays using each compound were performed in the absence of malate, testing for promiscuous oxidoreductase activity. None of the compounds notably increased the signal generated compared to assays performed in the absence of substrate (Figure 4.7).

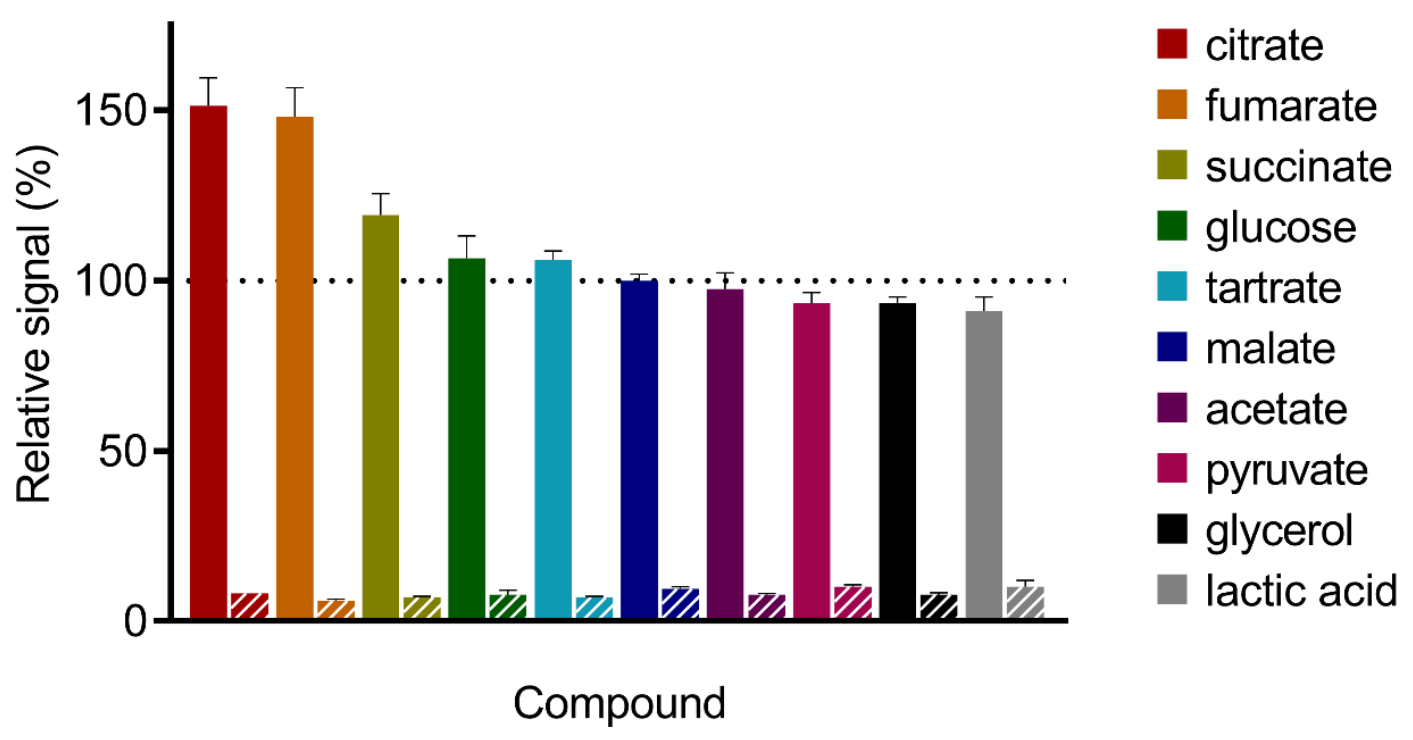

Figure 4.7. Testing various compounds for interference effects. Biosensor \#1 was used to obtain readings, with bars in block colour representing the output of assays containing $100 \mathrm{mM}$ of both the tested compound and malate. Bars have been ordered by the relative increase in signal. Striped bars denote the results of the same assays with malate absent. Error bars represent the standard error of technical triplicates.

To investigate the possibility of multiple compounds acting synergistically to increase the generated signal, each of the compounds shown in Figure 4.7 were all applied together to biosensor \#3 (MES-buffered), along with $5 \mathrm{mM}$ malate. Only $10 \mathrm{mM}$ of each compound was used, as opposed to $100 \mathrm{mM}$, due to the solubility limits of this mixture. Citrate was excluded due to already being used in the biosensor at $100 \mathrm{mM}$. The signal was reduced to $60 \% \pm 3 \%$ of that obtained with malate alone. Next, a blend of the activating compounds (from Figure 4.7) was made, comprising of fumarate and succinate at $20 \mathrm{mM}$, as well as the citrate (100 $\mathrm{mM}$ ) used in biosensor \#3. An even smaller signal was generated, at $27 \% \pm 3 \%$ of the original. 
Together, these experiments showed that some individual components of juice and wine were able to increase signal generation, although none of them approached the magnitude of the effect seen with actual juice samples (Figure 4.6). Unexpectedly, combinations of the compounds decreased AsME(R181Q) activity by substantially more than any individual compound. Therefore, none of the compounds tested in Figure 4.7 were responsible for the interference observed with juice and wine samples.

\subsubsection{Metal ions and amino acids}

Four of the most abundant amino acids in wine were tested for interference: glutamine, arginine, alanine and glutamic acid (Gutiérrez-Gamboa et al., 2019), in addition to various metal ions: $\mathrm{Ca}^{2+}, \mathrm{Na}^{+}, \mathrm{Zn}^{2+}$ and $\mathrm{K}^{+}$. The impact each of these compounds had on the biosensor signal is shown in Figure 4.8. While zinc precipitated when added to the assay mixture, $\mathrm{Na}^{+}$and $\mathrm{K}^{+}$caused minor increases in the signal generated (by $<10 \%$ ). Conversely, the addition of $\mathrm{Ca}^{2+}$ resulted in a reduction of the signal to $53 \% \pm 5 \%$ of the original. It was concluded that these compounds were not the main source of the interference.

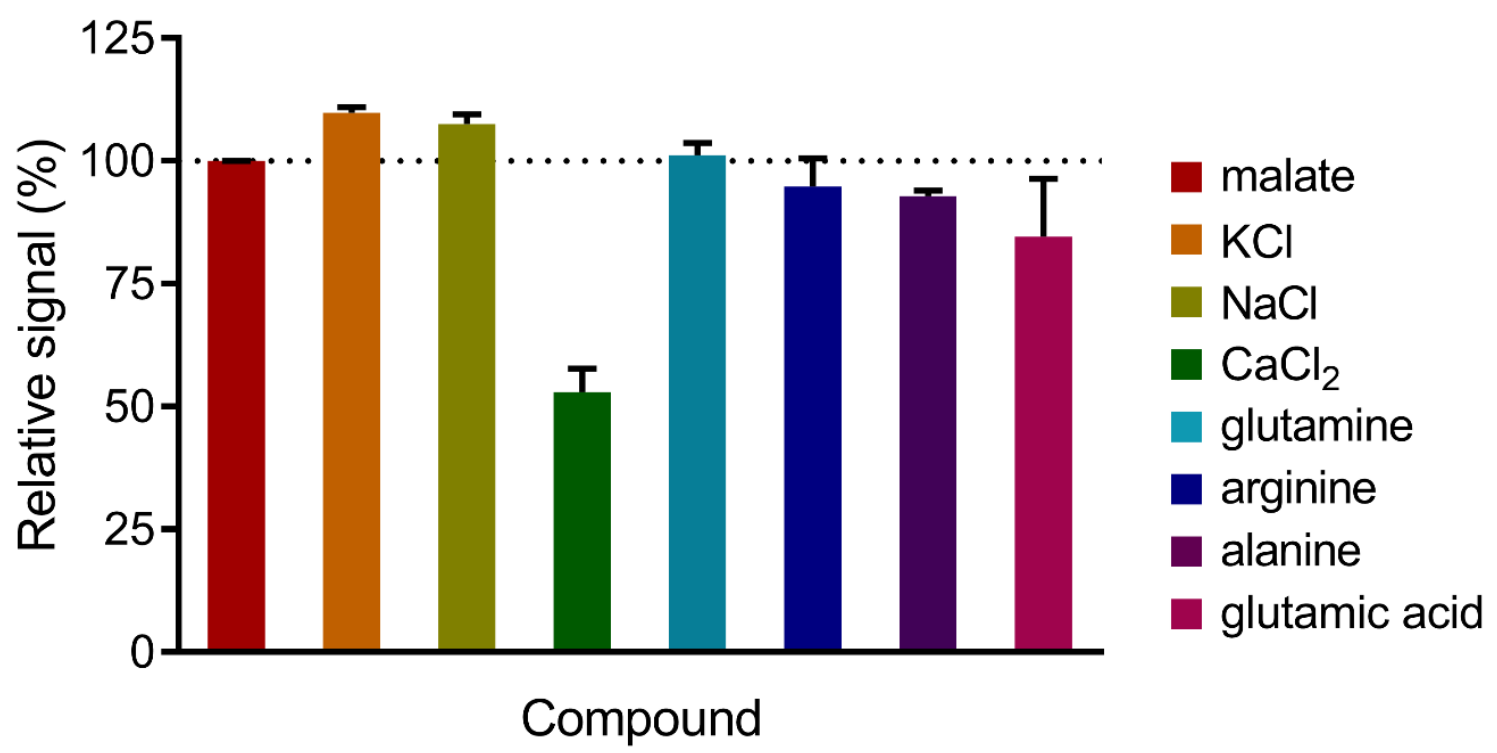

Figure 4.8. Testing various metal ions and amino acids for interference effects. Biosensor \#3 was used to obtain readings. Each assay contained $5 \mathrm{mM}$ malic acid, along with either $100 \mathrm{mM}$ metal ion or $7.5 \mathrm{mM}$ amino acid. Error bars represent the standard error of technical triplicates. 


\subsubsection{Phenolic compounds}

Polyphenols, found mainly in red wines, have been reported to interfere with various components of electrochemical biosensors (Chiorcea-Paquim et al., 2020, Gil and Rebelo, 2011). These can effectively be removed by activated carbon, a decolourisation process where polyphenols are adsorbed within hydrophobic layers of graphite (García-Pérez et al., 2019). The effect of decolourising samples on the interference signal is shown in Figure 4.9. As the interference is two-pronged, affecting both $\mathrm{AsME}(\mathrm{R} 181 \mathrm{Q})$ and $\mathrm{NAD}^{+} /$mediator, assays were performed with and without the enzyme to investigate the effects on each component.

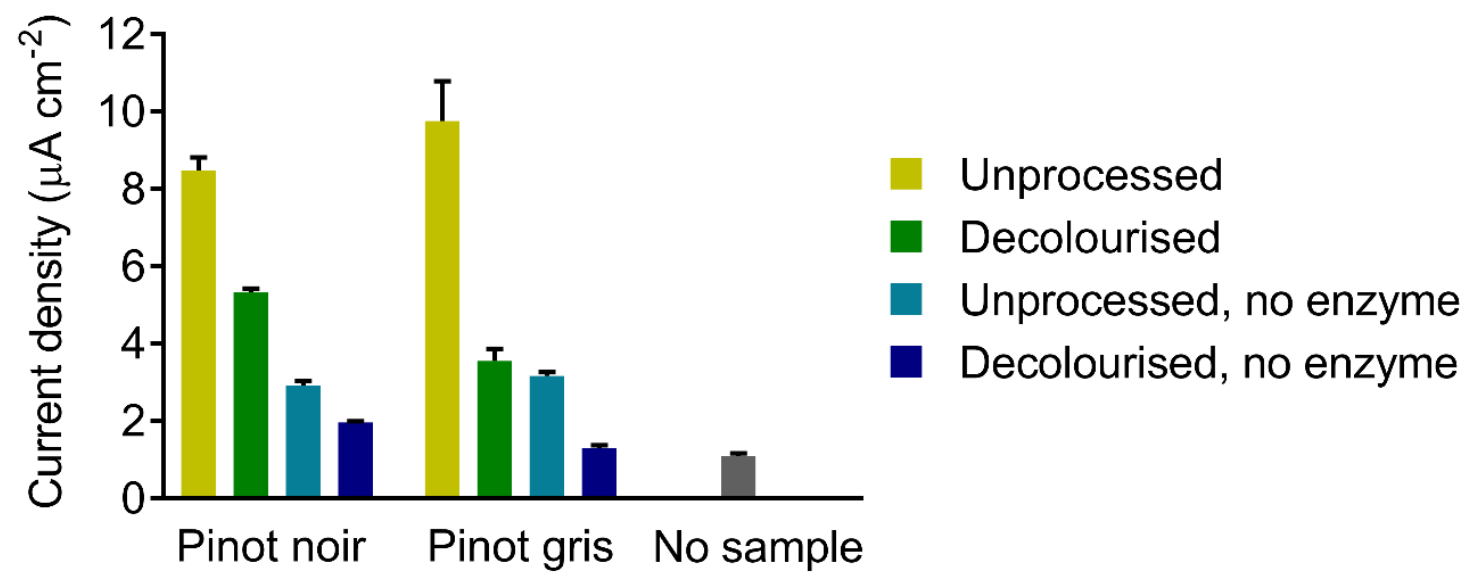

Figure 4.9. The effect of decolourising grape juice samples on the interference signal. Biosensor \#1 was used to obtain readings. Grape juice samples were sourced from the Hare's Run vineyard, and brought to $\mathrm{pH} 7 \mathrm{using}$ concentrated $\mathrm{NaOH}$. The 'no sample' reading is a standard assay in the absence of grape juice, which was substituted with water. Error bars represent the standard error of technical triplicates.

Such decolourisation initially appeared to be promising, marked by a decrease in current by $3.2 \mu \mathrm{A} \mathrm{cm}^{-2}$ for the Pinot Noir grape juice, and an even greater decrease when using Pinot Gris, $6.2 \mu \mathrm{A} \mathrm{cm}^{-2}$. In the absence of enzyme, decolourisation also decreased the interference signal by $0.9 \mu \mathrm{A} \mathrm{cm}^{-2}$ for Pinot Noir, and $1.9 \mu \mathrm{A} \mathrm{cm}^{-2}$ for Pinot Gris, suggesting that polyphenols, at least in part, were responsible for reducing $\mathrm{NAD}^{+} / \mathrm{DCPIP}$.

However, as determined by the measurements made using the Unitech commercial test kit, applying activated charcoal to samples also removes a portion of the malic acid. Comparisons of the percentage loss of malic acid with the percentage loss of enzyme-dependent signal 
(Table 4.3) shows the signal decrease to be disproportionate: a greater loss of signal occurs than the loss of malic acid. This suggests that decolourisation is useful for removing proportionately more of the interfering compound(s) than it removes malate, but the effects vary between grape variety (Table 4.3) making it difficult to deconvolute further, let alone to implement in a user-friendly device for winemakers.

Table 4.3. The effect of decolourisation on the malic acid concentrations within samples and the signals generated. Malic acid concentrations were determined by the Unitech commercial test kit. 'Enzymedependent' signals are shown to compare the percentage loss of signal with the percentage loss in malic acid after decolourising wine samples, shown in Figure 4.9. These were calculated as the difference between the signals generated using assays with enzyme and those without it. This accounts for the interference signal generated through the mediator and direct electron transfer to the electrode, which also decreases after decolourisation. Each concentration has been reported as the mean of triplicates, along with the standard error.

\section{Pinot Noir Pinot Gris}

$\begin{array}{lcc}\text { Malic acid concentration before decolourisation (mM) } & 22 \pm 1 & 24 \pm 2 \\ \text { Malic acid concentration after decolourisation (mM) } & 19 \pm 1 & 12+1 \\ \text { \% loss of malic acid from decolourisation } & \mathbf{1 6} & \mathbf{4 9}\end{array}$

$\begin{array}{lcc}\text { Enzyme-dependent signal before decolourisation }\left(\mu \mathrm{A} \mathrm{cm}^{-2}\right) & 5.5 \pm 0.3 & 6.6 \pm 1.1 \\ \text { Enzyme-dependent signal after decolourisation }\left(\mu \mathrm{A} \mathrm{cm}^{-2}\right) & 3.4 \pm 0.1 & 2.3 \pm 0.3 \\ \text { \% loss of enzyme-dependent signal from decolourisation } & \mathbf{3 8} & \mathbf{6 5}\end{array}$

\subsubsection{Ascorbic acid}

Initially, ascorbic acid was tested at a concentration of $5 \mathrm{mM}$, using biosensor \#3. The current readings obtained were approximately 10 -fold greater than the standard assays with malate alone, strongly suggesting that ascorbate contributed to the interference observed with juice and wine samples. Indeed, readings were similar with and without enzyme indicating that the ascorbate interference had completely saturated the sensor. Assays were repeated at a lower concentration of ascorbate $(0.1 \mathrm{mM})$, in an attempt to determine the nature of the interference signal (Figure 4.10). 


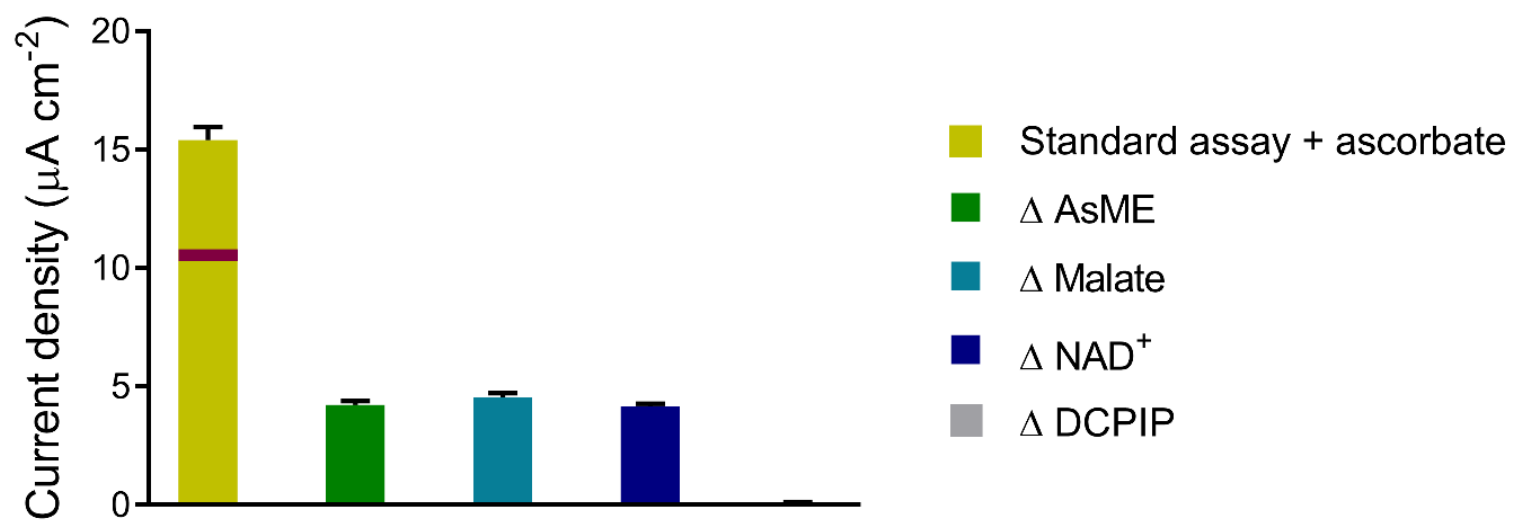

Figure 4.10. Experiments to deduce the nature of ascorbate interference. Biosensor \#1 was used to obtain measurements, with $0.1 \mathrm{mM}$ ascorbic acid and $100 \mathrm{mM}$ malate added to reaction mixtures. $(\Delta)$ symbols in the figure key indicate that the corresponding sensor component was removed. A red line has been drawn to show the signal of the assay in the absence of ascorbic acid, taken from the calibration plot of biosensor \#1 (Figure 3.6). Error bars shown represent the standard error of technical triplicates.

Standard assays with ascorbate produced current readings approximately $4 \mu \mathrm{A} \mathrm{cm}^{-2}$ greater than expected, despite the 1000-fold excess of malate over ascorbate. The same $4 \mu \mathrm{A} \mathrm{cm}^{-2}$ interference current is seen in the absence of enzyme, malate or $\mathrm{NAD}^{+}$, though is completely removed in the absence of DCPIP, showing that ascorbic acid specifically acts on the mediator (Figure 4.10).

The addition of iodine was explored as a strategy to mitigate the interference by oxidising ascorbic acid to the relatively inert dehydroascorbic acid (Caro-Jara et al., 2013). Crude assays showed that pre-treating ascorbate samples with iodine could be used to stop the reduction of DCPIP, as shown by the mediator remaining blue, as opposed to being turned clear - the colour of the reduced mediator. However, samples containing iodine were not compatible with the sensor, producing large cathodic (negative) currents, and produced a similar type of electrode fouling described for ferricyanide in section 3.2.1. Iodine was substituted for $\mathrm{CuCl}_{2}$ or $\mathrm{FeSO}_{4}$, which are both also capable of oxidising ascorbate to dehydroascorbic acid (Caro-Jara et al., 2013). However, these too were incompatible, as $\mathrm{CuCl}_{2}$ also produced large cathodic currents and $\mathrm{FeSO}_{4}$ resulted in a brown precipitate forming when added to assays, making the sensor unusable. 


\subsection{Discussion}

Initial tests showed that the sensors developed in chapter 3 were incompatible with grape juice/wine samples. Here, after first developing a buffering system to accommodate undiluted grape juice samples, I aimed to identify the factors preventing the accurate measurement of malate, then modify the sensor architecture to mitigate any hindrances.

\subsubsection{A buffering system for acidic samples}

Firstly, the acidity of grape juice/wine samples ( $\mathrm{pH} 3-4)$ overcame the original buffering system used by the sensor, $100 \mathrm{mM}$ HEPES $\mathrm{pH}$ 7. This resulted in the unwanted reduction of the mediator and diminished $\operatorname{AsME(R181Q)~activity.~As~described~in~section~4.1.2,~this~is~}$ seldom a problem for previously published malate biosensors due to the high dilution of samples in buffer before their application, performed to reduce the concentration of malate to accommodate narrow linear ranges. However, the sensors described here have been developed to have linear ranges that accommodate undiluted samples - increasing the influence of the grape juice's acidity.

Increasing the buffering capacity was a successful strategy for preventing large changes in $\mathrm{pH}$ upon addition of the sample. Various buffers were tested using an increased concentration of the buffering molecule, $500 \mathrm{mM}$, which generally prevented the $\mathrm{pH}$ from dropping below 0.5 units of the set $\mathrm{pH}$ of the buffer. For biosensor \#3, using MES buffer $\mathrm{pH} 6.7$, the range of these small changes in $\mathrm{pH}$ were sufficiently narrow to be within a stable range, $\mathrm{pH} 6.3$ - 6.7, where there was little change in the signal generated.

To the best of my knowledge, this is the first time that such an approach has been used for a biosensor designed to be used with acidic samples. The only other acidity mitigation strategy described in the malate biosensing literature is given by Maines et al. (2000), who used a plastic coating to protect the enzyme. One disadvantage of this system is the attenuation of the signal due to the coating causing reduced diffusion of analytes through the biosensor matrix. While some of the buffers tested in my study also attenuated the signal by destabilising certain assay reagents (e.g. those that used $500 \mathrm{mM}$ HEPES), it was relatively facile to test a range of buffer types, eventually arriving on $500 \mathrm{mM}$ MES pH 6.7, which produces a similar, though not identical, calibration curve to the original that used $100 \mathrm{mM}$ 
HEPES pH 7. It remains unclear how generalisable such a buffering system is, due to its dependence on a pH stable range, which may not be present in all amperometric biosensors. The $\mathrm{pH}$ dependencies of other biosensors are likely to vary considerably due to different $\mathrm{pH}$ optima described for malate oxidoreductases (Willeford and Wedding, 1987, Holaday and Gary, 1989) and mediators (Jahn et al., 2020), which together influence the overall pH dependence of a sensor. Accordingly, future work should first identify a $\mathrm{pH}$ stable range, if it exists, before developing the buffer component.

\subsubsection{Identifying the source of interference}

The biosensors developed here were susceptible to interference when using grape juice/wine samples: the signals produced were much greater than expected for the malic acid concentration present in samples, as determined by commercial testing kits. Collectively, the experiments in this chapter represent one of the most comprehensive investigations into compounds that may interfere with a malate biosensor, both in terms of the number of compounds tested and the efforts made to identify the specific components of the sensor affected.

The interference observed is a large anodic current inducted by multiple components of the sensor: the enzyme itself, as well as downstream components (either/both $\mathrm{NAD}^{+}$and the mediator). Direct electron-transfer to the electrode was not a significant source of interference.

Theoretically, two mechanisms of enzyme-based interference exist: 1 . the enzyme catalyses a non-specific reaction producing a molecule capable of reducing the mediator; and 2. the rate of malate oxidation is increased or decreased in the presence of the interfering molecule. As assays in the absence of malate failed to generate a signal, the latter was true for the interfering compounds shown in Figure 4.7.

Here, citrate, fumarate and succinate have been shown to enhance the signal produced by the biosensor. For fumarate and succinate, greater signals were only seen at the relatively high concentration of $100 \mathrm{mM}$. Lower concentrations tested in chapter $3(\leq 10 \mathrm{mM}$ for fumarate, and $\leq 50 \mathrm{mM}$ for succinate), did not change the signal. This insensitivity to activating compounds is beneficial to the sensor, as the low concentrations of fumarate and succinate 
found in wine and grape juice do not considerably affect the signal. These are less than 10 $\mathrm{mM}$ for succinate (Table 4.1), while concentrations of fumarate in grape/juice have not been reported, assumedly because it exists only in trace amounts, or not at all. Citrate too, is a relatively minor constituent of wine, typically found at concentrations $<1 \mathrm{mM}$ (Soyer et al., 2003). As shown by the citrate optimisation experiments for biosensor \#3 in Chapter 3 (Figure 3.8), such concentrations are likely to have little impact on the signal.

The effect of polyphenols on sensor performance remains inconclusive. Though a reduction in both the enzyme- and mediator- dependent interference signals occurs when samples are decolourised (Figure 4.9), so does the malic acid concentration, making it likely that other small molecules are also adsorbed by the activated carbon. As these could include nonpolyphenol interfering molecules, the interference effects of polyphenols remain unclear. Alternative dephenolisation strategies include absorption by polyvinylpyrrolidone (Andersen and Sowers, 1968), which is used in the malic acid testing kit from Megazyme (https://www.megazyme.com/), or the use of a tyrosinase enzyme to catalyse the degradation of polyphenols into removable products (Girelli et al., 2006). However, for both techniques, the specificity towards other compounds in wine is unknown.

Ascorbic acid is a potent interferent for the biosensor, affecting the mediator. This is perhaps unsurprising since the molecule partakes in a well-known reaction with DCPIP (Omaye et al., 1979). Ascorbic acid has been reported to exist a small concentrations within grape juice, approximately $75 \mu \mathrm{M}$, before it is rapidly degraded to trace amounts during fermentation (Ribéreau-Gayon et al., 2006). The concentrations of ascorbic acid used in my study, 100 $\mu \mathrm{M}$, is similar to that found grapes (Table 4.1), and produces a $4 \mu \mathrm{M} \mathrm{cm}^{-2}$ interference signal that corresponds well to the "NAD"/mediator interference" seen in Figure 4.6. As ascorbic acid degrades during the fermentation process (Ribéreau-Gayon et al., 2006), it would be expected that the level of interference would decrease across process the time point experiments shown in Figure 4.5. However, this is not the case, which suggests that ascorbate is not a main source of interference in real samples, but is rather another compound(s).

To test this hypothesis, attempts were made to remove ascorbate from grape juice samples to investigate if this decreased the interference signal. The use of iodine for its removal has been used previously for a malate biosensor (Mundaca-Uribe et al., 2017), though it is incompatible with the sensor in this current study. The use of ascorbate oxidase, which similarly converts ascorbic acid to dehydroascorbic acid has been shown to be an effective 
means of pre-treatment two malic acid biosensors (Maines et al., 2000, Manzoli et al., 2004) and may be easier to integrate into this enzyme-centric architecture. At the time of writing (September 2021), ascorbate oxidase from Cucurbita sp. is readily available as a commercial preparation from Sigma-Aldrich.

In Figure 4.6, "NAD + mediator interference" accounted for approximately one third of the interference, while the rest was caused by a different type of interference that affected AsME(R181Q) activity. Ascorbate solely affected the mediator, meaning the compound(s) causing the latter type of interference are yet to be found. For further testing of individual compounds, expanding the range to include those found in glycolysis and the citric acid cycle maybe prove fruitful, as malic enzyme acts at the interface of these two processes (Kanellis and Roubelakis - Angelakis, 1993), with specific compounds from each pathway having been reported to modulate activity of certain MEs (Sato et al., 2010, Bologna et al., 2007, Su et al., 2009). 


\section{Chapter Five-Summary, conclusions, and future work}

\subsection{Summary and conclusions}

Prior to this work, 40 malic acid biosensors had been described in the published literature, as reviewed over the course of this project (Chapter 1 and (Matthews et al., 2021)). While much effort has been directed into improving sensor performance by way of the electron mediator, immobilisation method and modifications to the electrode surface, there has been a general lack of focus on the choice of enzyme. Here, I sought to show that a bespoke enzyme can be used to simultaneously improve the attributes of a biosensor and afford new functionality. To guide my efforts, I aimed to solve a problem prevalent in the literature - narrow linear ranges that are poorly suited to the concentrations of malic acid found in real samples, specifically those found in winemaking, a process which has driven much of the malate biosensor research. I aimed to extend the linear range by using a malate oxidoreductase enzyme with a high $K_{\mathrm{M}}$ for malate.

The search for the enzyme to be used in our device started bioinformatically, using the BRENDA database (https://www.brenda-enzymes.org/). This repository was particularly well-suited for this project, as it not only curates hundreds of text-mined $K_{\mathrm{M}}$ values but also a wide range of functional and molecular properties. This allowed for a dual selection criterion: enzyme candidates must have a high $K_{\mathrm{M}}$, as well as having other functional characteristics or molecular parameters that may benefit a biosensor. Other databases that were considered do not have such a holistic approach to the data collected, instead focusing one aspect of the enzyme. For example, the SABIO-RK database only focuses on the enzyme kinetics (Wittig et al., 2012).

The search was successful, and led to the creation of a shortlist of six candidates. These enzymes were subjected to a screening process, where they were expressed, purified and subjected to characterisation through spectrophotometric assays. A lead candidate, $\operatorname{AsME}(\mathrm{R} 181 \mathrm{Q})$ was identified that is easy to produce and could produce linear enzyme kinetics up to $200 \mathrm{mM}$ malic acid, the maximum concentration found in grapes (Volschenk et 
$a l ., 2006)$. This enzyme was chosen, in part, due to the previously-described ability to modify apparent kinetic parameters by varying the concentration of ammonium used in assays (Karsten and Cook, 2007). However, this system was deemed redundant after developing a new assay that used high concentrations of $\mathrm{Mg}^{2+}$. The idea for a tuneable mechanism was later revitalised when it was found that the kinetic behaviour could be modified by switching the divalent metal ion used in assays and by adding citrate to assays.

Next, AsME(R181Q) was paired with an electrode and electron mediator to transduce the catalytic reaction into a measurable electrical signal. It was known that the choice of the electron mediator can have profound effects on the performance of biosensors, and so six different mediators were screened. Few were suitable, with many being unstable or the cause of electrode fouling. DCPIP was chosen due to its relatively low operating potential and ability to generate the highest current out of all the mediator candidates. However, despite being the best mediator in this study, there were some limitations to its use: its poor solubility prevented the use of optimal concentrations, and its poor stability when dissolved in the buffers used made assays more complicated to perform. Expanding the range of mediators tested may prove fruitful in solving both issues.

The enzyme kinetics of AsME(R181Q), determined spectrophotometrically, translated well into creating an amperometric sensor with a wide linear range. The range of malic acid concentrations accommodated by the device is the widest described to date, with calibration curves being linear to $200 \mathrm{mM}$, similar to the plots produced spectrophotometrically. Likewise, additives that modulated the enzyme kinetics were used to change the operational parameters of the biosensor. However, some differences were observed between electrochemical and spectrophotometric assays, such as unexpectedly high signals when using certain concentrations of malate. In conclusion, a bespoke enzyme chosen for its kinetic behaviour has a profound influence on the operational parameters, but when integrated into a biosensor architecture, such as the relatively simple one used here, the operational parameters of the sensor can deviate from the observed kinetic behaviour.

Here, for the first time, an enzyme-based biosensor with a dynamic sensitivity versus linear range is described. In Chapter 3, I showed how a single device can be used to accommodate the differing needs of winemakers, both for processes where small and large differences in malic acid concentration are important. My results are a testament to the idea that the enzyme-centric design of a biosensor is a fruitful avenue for research that aims to improve on 
current malate-sensing devices. Future work may capitalise on enzymes with different features, such as those of candidate enzymes removed by the screen. These include unique modes of regulating activity (i.e. the on/off switch seen for SvMDH, (Lemaire et al., 1996)), high stability (pH-stable and thermostable enzymes) and enzymes that do not require $\mathrm{NAD}(\mathrm{P})^{+}$(i.e. the malate quinone oxidoreductases (Kretzschmar et al., 2002)). For endeavours that do aim to create the most sensitive device possible, selecting enzymes based on high catalytic efficiency $\left(k_{\mathrm{cat}} / K_{\mathrm{M}}\right)$ may be key.

In terms of applying real samples to sensor, two aspects were addressed: 1 . mitigating the effects of the acidic wine and grape juice samples on enzyme activity; and 2. identifying the source of interference. The former was resolved by developing a buffering system that used a high concentration of MES buffer and a tuned initial buffer $\mathrm{pH}$ so that sensor assays remain within a " $\mathrm{pH}$ stable" range once the sample is added; that is, a $\mathrm{pH}$ range where the signal remained consistent. This strategy proved effective for the samples used in this study. Further, a previous approach using a protective membranes to regulate $\mathrm{pH}$ (Maines et al. 2000) decreased readings, whereas the use of MES buffer here actually increased sensitivity.

Real samples consistently produced an interference signal that manifested as a large anodic current, affecting both the enzyme and the $\mathrm{NAD}^{+} /$mediator. Many different compounds found in wine were assessed for their role as a potential interferent, and ascorbic acid was identified as a potent source of non-specific current acting through the mediator, DCPIP. Though almost all the main compounds in wine were tested, the interferent capable of modulating enzyme activity remains elusive. While further experiments are required to assess the effect of ascorbate across the range of concentrations it is found in juice and wine, it may be responsible for at least $33 \%$ of the interference signal. The complexity of wine extends far beyond the constituents found at highest concentrations, tested in this study, with the activity of certain malate oxidoreductases having been reported to be influenced by far smaller concentrations of specific compounds. For example, both the $K_{\mathrm{M}}$ and $k_{\text {cat }}$ of AcME are profoundly influenced by as little as $25 \mu \mathrm{M}$ acetyl-CoA (Zhang et al., 2012). As this adds hundreds of compounds found in wine to be tested, future work may benefit from simplifying real samples by fractionation and the use of powerful analytical tools to identify specific molecules, such as mass spectrometry. 


\subsection{Towards creating a useable sensor}

Several factors must be addressed before this biosensor constitutes a useable product. Most importantly, the interferents must be found and dealt with. One interferent, ascorbic acid, has been identified, though the attempts to remove it using chemical additives have been unsuccessful. Immediate future work should therefore experiment with the use of the ascorbate oxidate enzyme, which catalyses the degradation of ascorbic acid into the inert dehydroascorbic acid. However, even if this enzyme-based method also fails to remove this interferent, the sensor system described here can still find use within a spectrometric assaybased kit, as this eliminates the need for the electron mediator, DCPIP - the target of ascorbic acid interference.

Storage stability and response time are two parameters that were not the focus of this work. However, both are important for the commercial viability of amperometric sensors (see sections 1.8 and 1.9 for discussions of each parameter). As described in chapter 1 , the ability to be stored for six months is considered the minimum requirement for a marketable sensor. The time constraints on this project have prevented such a long-term experiment. This type of study would most aptly be performed once a final sensor has been created, i.e. one that may contain the mechanism for eliminating interference and immobilised reagents on the electrode.

Currently, the response time of the device is two minutes and five seconds. While this is a typical time amongst malic acid biosensors, it represents a relatively long period in comparison to commercially available glucose sensors, which are able to produce readings within 5-15 s. For the device described here, attempts at reducing the response time should first focus on reducing the incubation step once the sample has been added. Care should be taken so as not to diminish the sensitivity of the device due to a reduced time-dependent production of NADH by AsME(R181Q), though increasing the enzyme concentration used on the electrode surface may remediate this.

The device described here has the potential to enter the US\$105-350M (Kövilein et al., 2020) market for malic acid with a unique selling point of being able to measure both large and small differences concentration. The biosensor has been designed to be used in winemaking, a US\$287B industry (Anderson et al., 2017), and is operationally flexible to accommodate a wide range of winemaker needs. Overall, the device represents a valuable, new concept that 
will help amperometric malic acid biosensors gain a commercial footing, similar to that achieved by glucose sensors.

\subsection{General conclusions}

Despite unreliable readings using real samples, this study remains a strong proof of principle - that the careful consideration of which enzyme is used to construct a biosensor holds (previously) untapped potential for creating improved and novel biosensors. We have shown here that a bespoke enzyme can be used to extend the linear range of a biosensor and afford a mechanism for dynamically modulating the sensor's operational parameters, though there are doubtless many other characteristics of enzymes that we have not considered that could be exploited in a similar manner as done here. With the availability of comprehensive enzyme databases and the ease of gene synthesis, the use of bespoke enzymes in biosensors is a strategy ripe for further exploration. 


\section{Chapter Six- Materials and Methods}

\subsection{Materials}

All chemicals used in this study were purchased at reagent or laboratory grade purity. Unless otherwise stated, these were sourced from Sigma Aldrich (St Louis, MO, USA). Solutions of reagents were generally made using deionised water, though, as noted in the following sections, some techniques used ultrapure water from a Barnstead Easypure II water purification System (Lake Balboa, CA, USA). Where needed, solutions were autoclaved at $121^{\circ} \mathrm{C}, 100 \mathrm{kPa}$ for $20 \mathrm{~min}$.

\subsubsection{DNA}

The sequences corresponding to each malate oxidoreductase were sourced from various databases. These are shown in Table 6.1, along with the accession code. For this study, the sequences of SvMDH and AsMDH were modified to remove the first 120 and 12 amino acids, respectively, as these are not present in the mature protein products (Kulkarni et al., 1993, Crétin et al., 1990). 
Table 6.1. The malate oxidoreductase genes used in this study

\begin{tabular}{|c|c|c|}
\hline Malate oxidoreductase $^{a}$ & Gene sequence database & Accession code $^{b}$ \\
\hline $\begin{array}{c}\text { Azorhizobium caulinodans, } \\
\text { NAD } \\
\text { (1.1.1.39) }\end{array}$ & KEGG genes & AZC_3656 \\
\hline $\begin{array}{c}\text { Ascaris suum, } \mathrm{NAD}^{+} \text {- dependent } \\
\text { malic enzyme, R181Q mutant } \\
(1.1 .1 .39)\end{array}$ & GenBank & M81055.1 \\
\hline $\begin{array}{c}\text { Sorghum vulgare NADP+ } \\
\text { dependent malate dehydrogenase } \\
(1.1 .1 .82)\end{array}$ & GenBank & X53453.1 \\
\hline $\begin{array}{c}\text { Aeropyrum pernix, NAD(P) })^{+} \\
\text {dependent malate dehydrogenase } \\
(1.1 .1 .82)\end{array}$ & GenBank & AB263815.1 \\
\hline $\begin{array}{l}\text { Thermotoga maritima, putative } \\
\text { malic enzyme }\end{array}$ & RefSeq & NP_228352.1 \\
\hline $\begin{array}{c}\text { Escherichia coli, } \mathrm{NAD}(\mathrm{P})^{+} \text {malic } \\
\text { enzyme }(1.1 .1 .38)\end{array}$ & GenBank & $\begin{array}{l}\text { U00096.3, } \\
\text { b1479 }\end{array}$ \\
\hline
\end{tabular}

\footnotetext{
a Malate oxidoreductases highlighted in bold typeface were identified using BRENDA. Remaining enzymes were 'in-house' variants sourced from our lab.

b 00096.3 refers to the accession code for the complete genome Escherichia coli str. K-12 substr. MG1655. b1479 is the Blattner identifier for the gene encoding MaeA.
}

The genes encoding AcME, AsME, SvMDH and ApMDH were synthesized and cloned into the pET-28 a (+) vector by Twist Bioscience (San Francisco, CA, USA). DNA was received as a lyophilised powder, which was resuspended in TE buffer (10 mM Tris- $\mathrm{HCl}, 0.1 \mathrm{mM}$ EDTA, $\mathrm{pH}$ 8.0) to a final concentration of $10 \mathrm{ng} / \mu \mathrm{L}$. When not being used, DNA samples were stored at $-20^{\circ} \mathrm{C}$.

The genes encoding MaeA was sourced from the ASKA (A complete Set of E. coli $\mathbf{K}-12$ ORF Archive) collection, which is a complete set of $E$. coli open reading frames cloned into the pCA24N expression vector (Kitagawa et al., 2005). The gene encoding the TmME enzyme was sourced from a library of open reading frames from Thermotoga maritima that have been inserted into the pMH1 vector (Lesley et al., 2002). The plasmids used in this study, and their important features, are shown in Table 6.2. 
Table 6.2. Plasmids used in this study.

\begin{tabular}{cccc} 
Name & Antibiotic resistance & Inducer for expression & Promoter \\
\hline pET-28 a $(+)$ & Kanamycin & IPTG & T7 \\
pMH1 & Ampicillin & Arabinose & araC \\
pCA24N & Chloramphenicol & IPTG & T5-lac \\
\hline
\end{tabular}

\subsubsection{Bacterial strains}

Two strains of $E$. coli were used throughout this study. These are described in Table 6.3.

Table 6.3. E. coli strains used in this study.

\begin{tabular}{|c|c|c|}
\hline Strain & Genotype & Source \\
\hline E. cloni $10 \mathrm{G}$ & $\begin{array}{c}\text { F- } m c r A \Delta(m r r-h s d \mathrm{RMS}-m c r B C) \text { endA1 recA1 } \$ 80 \text { dlacZ } \Delta \mathrm{M} 15 \\
\Delta \text { lacX74 araD139 } \Delta \text { (ara,leu)7697galU galK rpsL nupG } \lambda- \\
\text { tonA (StrR) }\end{array}$ & $\begin{array}{c}\text { Lucigen } \\
\text { (Middletown, WI, USA) }\end{array}$ \\
\hline BL21(DE3) Gold & $\begin{array}{c}\text { E. coli B F- ompT hsdS }\left(\mathrm{rB}^{-} \mathrm{mB}^{-}\right) d c m^{+} \operatorname{Tet}^{r} g a l \lambda(\mathrm{DE} 3) \text { endA } \\
\text { Hte }\end{array}$ & $\begin{array}{l}\text { Agilent technologies (Santa Clara, } \\
\text { CA, USA) }\end{array}$ \\
\hline
\end{tabular}

\subsubsection{Supplements}

Various supplements were used throughout the study. Antibiotics were employed to select for bacteria that contained the desired plasmid DNA. Kanamycin, ampicillin and chloramphenicol were made as $1000 \times$ stock solutions using ultrapure water, or $100 \%$ ethanol for chloramphenicol, at concentrations of $30 \mathrm{mg} / \mathrm{mL}, 100 \mathrm{mg} / \mathrm{mL}$ and $34 \mathrm{mg} / \mathrm{mL}$, respectively. The working concentration for each was $1 \times$. Both IPTG and arabinose were used to induce protein overexpression. Generally, concentrations of $1 \mathrm{mM}$ and $0.1 \%(\mathrm{w} / \mathrm{v})$ were used for IPTG and arabinose, respectively, though these were often varied during attempts to optimise protein expression. All supplement stock solutions were filtered sterilised using syringe-driven sterile filters with $0.22 \mu \mathrm{M}$ pore size (Jet Biofil, Guangzhou, China). 


\subsection{DNA Manipulation}

\subsubsection{DNA electrophoresis}

DNA electrophoresis was used to separate and visualise DNA fragments by size. The separation matrix consisted of $1 \%(\mathrm{w} / \mathrm{v})$ agarose dissolved in TAE buffer (40 mM Tris base, $20 \mathrm{mM}$ acetic acid, $1 \mathrm{mM}$ EDTA, pH 8.0), and was casted as a layer approximately $1 \mathrm{~cm}$ thick with a comb inserted to form wells as it set.

Gels were inserted into a Mini-Sub Cell GT horizontal cell (Bio-Rad, Hercules, CA, USA) and immersed in TAE buffer which acted as the electrolyte. Before loading, samples were mixed with a $1 \times$ working concentration of KAPA loading dye, and were run alongside KAPA universal DNA ladder for size comparison (both from KAPA Biosystems, Wilmington, MA, USA). Electrophoresis used $100 \mathrm{~V}$ for approximately $30 \mathrm{~min}$. Visualisation of DNA fragments began with soaking gels in $0.01 \%(\mathrm{v} / \mathrm{v})$ ethidium bromide solution for $1 \mathrm{~h}$, then rinsing them with water. The remaining intercalated dye was then illuminated using ultraviolet (UV) light. As UV light damages DNA, fragments that were required for future cloning experiments were instead visualised under blue light, though this required SYBR Safe stain (Thermo Fisher Scientific, Waltham, MA, USA) to be added to gel mixtures instead of soaking them in ethidium bromide. Gels were imaged using the UVITEC Essential V6 imaging platform (United Kingdom).

\subsubsection{DNA purification}

Separated DNA fragments from electrophoresis (see section 6.2.1) were excised from agarose gels using a scalpel, then purified using the NucleoSpin Gel and PCR Clean-up kit from New England BioLabs (Ipswich, MA, USA). The supplied buffer used to elute DNA from the clean-up columns was pre-heated to $70^{\circ} \mathrm{C}$ to improve yield. Estimations of the concentration of the purified DNA used the absorbance at $260 \mathrm{~nm}$, performed by the Eppendorf (Hamburg, Germany) BioPhotometer Model \#6131, before samples were stored at $4^{\circ} \mathrm{C}$. 


\subsubsection{Plasmid extraction}

Plasmid preparation was performed using the Monarch Plasmid DNA Miniprep Kit (Ipswich, MA, USA), following the protocol provided by the manufacturer. The procedure used $5 \mathrm{~mL}$ of overnight culture grown at $37^{\circ} \mathrm{C}$, and DNA was eluted using $40 \mu \mathrm{L}$ of the supplied elution buffer that had been pre-warmed to $50^{\circ} \mathrm{C}$. The concentration was estimated as is described in section 6.2.2.

\subsubsection{Restriction cloning}

Restriction enzyme-based excision was used in this study to remove the GFP-tag from the gene encoding MaeA. Plasmids were extracted from E. cloni 10G containing pCA24N-maeA (+ GFP) as is described in section 6.2.3. Largely following the protocol given by the New England BioLabs NEBcloner tool (https://international.neb.com/), the NotI-HF restriction endonuclease was used to cleave recognition sites that flank both the $5^{\prime}$-and $3^{\prime}$ ends of the sequence encoding GFP. The $30 \mu \mathrm{L}$ reaction mixture is shown in Table 6.4.

Table 6.4. Restriction digest mixture.

\begin{tabular}{ccc} 
Reagent & Volume $(\mu \mathrm{L})$ & Working Concentration \\
\hline CutSmart Buffer $(10 \mathrm{x})$ & $3 \mu \mathrm{L}$ & $1 \times$ \\
Notl-HF $(20 \mathrm{U} / \mu \mathrm{L})$ & $1 \mu \mathrm{L}$ & $0.7 \mathrm{U} / \mu \mathrm{L}$ \\
$\mathrm{DNA}$ & Varied & $\sim 70 \mathrm{ng} / \mu \mathrm{L}$ \\
$\mathrm{H}_{2} \mathrm{O}$ & & - \\
\hline
\end{tabular}

Both Notl-HF and the CutSmart Buffer were purchased from New England Biolabs (Ipswich, MA, USA).

Digests were run overnight $(\sim 16 \mathrm{~h})$ at $37^{\circ} \mathrm{C}$. DNA products where then separated by electrophoresis (see section 6.2.1), resolving the double-cut vector from the single-cut and uncut. A band corresponding to $6215 \mathrm{bp}$ for the gene encoding MaeA(- GFP) was excised and purified as described in section 6.2.2. 
Plasmids were then re-circularised using T4 DNA ligase in the reaction mix shown in Table 6.5. The method used is a modified version of the 'Ligation Protocol with T4 DNA Ligase', given by New England BioLabs (https://international.neb.com/), using a low concentration of DNA so that intramolecular ligation is favoured over concatemerisation. The reaction was run at $16^{\circ} \mathrm{C}$ overnight, before being use to transform electrocompetent E. cloni $10 \mathrm{G}$ and BL21(DE3) Gold E. coli cells. The protocol used for transformation is described in section 6.3.3. Transformed cells using the ligation mixture were checked for the - GFP vector by diagnostic restriction digest, confirmed by the absence of a $772 \mathrm{bp}$ band when using NotI-HF.

Table 6.5. Ligation mixture

\begin{tabular}{ccc} 
Reagent & Volume $(\mu \mathrm{l})$ & Working Concentration \\
\hline Ligation buffer $(10 \mathrm{x})$ & $1 \mu \mathrm{L}$ & $40 \mathrm{U} / \mu \mathrm{L}$ \\
\hline T4 DNA ligase $(400 \mathrm{U} / \mu \mathrm{L})$ & $1 \mu \mathrm{L}$ & $2 \mathrm{ng} / \mu \mathrm{L}$ \\
Linearised plasmid & Varied & - \\
$\mathrm{H}_{2} \mathrm{O}$ & To $10 \mu \mathrm{L}$ & \\
\hline Both T4 DNA ligase and the ligation buffer were purchased from New England Biolabs (Ipswich, MA, USA).
\end{tabular}

\subsection{Microbiological techniques}

\subsubsection{Preparation of starter cultures}

Initiating a starter culture was among the first steps of many of the microbial-based techniques described below. These were created by first streaking lysogeny broth (LB)-agar with $E$. coli freezer stocks, then picking one colony to inoculate $5 \mathrm{~mL}$ LB. In most cases, the appropriate antibiotic was added at each stage to prevent contamination. Inoculated starter cultures were grown overnight at $37^{\circ} \mathrm{C}$, shaking at $200 \mathrm{rpm}$, before being used. 


\subsubsection{Preparation of electrocompetent $E$. coli}

Electrocompetent cells were used in this study for high efficiency cloning. The preparation procedure began with creating a starter culture (section 6.3.1) for the desired strain of E. coli, either E. cloni 10G or BL21(DE3) Gold. As neither strain contains an antibiotic resistance marker, LB-agar plates used for streaking and the inoculated liquid medium contained no antibiotic.

The starter culture was used to inoculate $500 \mathrm{~mL} \mathrm{LB}$, which was then incubated at $37^{\circ} \mathrm{C}$, while shaking at $200 \mathrm{rpm}$, until the optical density (OD) reached 0.4 . The culture was subsequently split into $50 \mathrm{~mL}$ aliquots, then cooled on ice. Cells were pelleted by centrifugation at $4^{\circ} \mathrm{C}, 1,800 \mathrm{~g}$, for $10 \mathrm{~min}$, before discarding the supernatant.

Pelleted cells were next subjected to a series of wash steps: twice, the pellets were gently resuspended in chilled $\mathrm{H}_{2} \mathrm{O}$, before being repelleted and the supernatant discarded. The final wash used $10 \%$ glycerol instead of $\mathrm{H}_{2} \mathrm{O}$, with the pellet being resuspended in residual liquid after the supernatant was decanted. This suspension was then split into pre-chilled microcentrifuge tubes as $50 \mu \mathrm{L}$ aliquots, then stored at $-80^{\circ} \mathrm{C}$.

\subsubsection{Transformation of electrocompetent cells}

An aliquot of electrocompetent cells (section 6.3.2) was first thawed on ice before being gently mixed with $1 \mu \mathrm{L}$ of the ligation reaction or commercially prepared plasmid. After a 10 min incubation on ice, the mixture was transferred into a sterile $2 \mathrm{~mm}$ MicroPulser electroporation cuvette (Bio-Rad, Hercules, CA, USA). Electroporation was performed using the MicroPulser electroporator (Bio-Rad, Hercules, CA, USA), which pulsed a voltage of 2.5 $\mathrm{kV}$. Transformed cells were then recovered in $500 \mu \mathrm{L} \mathrm{SOC}$ medium, at $37^{\circ} \mathrm{C}$ while shaking, for $1 \mathrm{~h}$. Between $5-50 \mu \mathrm{L}$ of the recovery mixture was spread on LB-agar plates containing the appropriate antibiotic. Colonies were grown overnight at $37^{\circ} \mathrm{C}$. 


\subsubsection{Preparation of frozen stocks for long-term storage}

Colonies of transformed bacteria were used to create overnight cultures. These were then mixed in a 1:1 ratio with $50 \%(\mathrm{w} / \mathrm{v})$ glycerol before being aliquoted in $1 \mathrm{~mL}$ cryogenic storage vials from Sigma (St Louis, MO, USA) and stored at $-80^{\circ} \mathrm{C}$.

\subsection{Protein manipulation}

\subsubsection{SDS-PAGE}

SDS-PAGE (sodium dodecyl sulfate polyacrylamide gel electrophoresis) was used to visualise proteins by their difference in size. SDS-PAGE gels consist of a stacking and resolving layer. Their make-up is shown in Table 6.6.

Table 6.6. The recipes used to create 10 SDS-PAGE gels.

\begin{tabular}{ccc} 
Reagent & Stacking Gel (4\%) & Resolving Gel (12\%) $^{\mathbf{b}}$ \\
\hline SDS $(10 \% \mathrm{w} / \mathrm{v})$ & $200 \mu \mathrm{L}$ & $550 \mu \mathrm{L}$ \\
Acrylamide/Bis-acrylamide $(40 \% \mathrm{w} / \mathrm{v})$ & $2 \mathrm{~mL}$ & $16.5 \mathrm{~mL}$ \\
Tris-HCla & $5 \mathrm{~mL}$ & $13.75 \mathrm{~mL}$ \\
Ammonium persulfate $(10 \% \mathrm{w} / \mathrm{v})$ & $100 \mu \mathrm{L}$ & $275 \mu \mathrm{L}$ \\
$\mathrm{H}_{2} \mathrm{O}$ & $12.7 \mathrm{~mL}$ & $23.9 \mathrm{~mL}$ \\
$\mathrm{~N}, \mathrm{~N}, \mathrm{~N}^{\prime}, \mathrm{N}^{\prime}$-tetra-methylethylenediamine (TEMED) & $20 \mu \mathrm{L}$ & $55 \mathrm{~L}$ \\
\hline
\end{tabular}

${ }^{a} 0.5 \mathrm{M}$ Tris- $\mathrm{HCl}, \mathrm{pH}=6.8$ was used for stacking gels, $1.5 \mathrm{M}$ Tris- $\mathrm{HCl} ; \mathrm{pH}=8.8$ was used for resolving.

${ }^{b}$ Percentages refer to the $w / v$ of acrylamide/bis-acrylamide in gels. 
TEMED was added last to start the polymerisation reaction that causes the gels to set.

Mixtures for each gel type were cast in between two glass plates with $1 \mathrm{~mm}$ spacing, with the resolving gel forming the bottom layer, and the stacking gel creating an approximately $2 \mathrm{~cm}$ thick layer on top. A gel comb was inserted into the stacking layer before it had set, creating wells to accommodate protein samples. Fully polymerised gels were stored in their glass casing at $4^{\circ} \mathrm{C}$, wrapped in a moistened paper towel, and sealed in an airtight plastic bag.

In preparation for electrophoresis, protein samples were mixed in a 1:1 ratio with SDS loading dye, then were incubated at $95^{\circ} \mathrm{C}$ for $10 \mathrm{~min}$. Both the high temperature and the buffer act to denature the protein, preventing the protein's folded structure from affecting its migration through the gel. The constituents of the dye, as well as other solutions used in SDSPAGE analysis can be found Table 6.7.

Electrophoresis was performed using the Bio-Rad mini-PROTEAN Tetra Vertical Electrophoresis Cell (Hercules, CA, USA): gels were placed in a two-well cassette, with a buffer dam being used if running only one gel. Cassettes were fitted into the buffer tank, which in turn, was filled with running buffer. Typically, $10 \mu \mathrm{L}$ of the protein plus loading dye mixture was added to the gel wells, though less volume was used if it was known that sample contained a particularly high concentration of protein. Samples were run at $180 \mathrm{~V}$ for $1 \mathrm{~h}$, alongside the Precision Plus Protein prestained ladder, which is a series of molecular weight standards for size comparison. Gels were subsequently stained using a Coomassie blue-based mixture for at least $30 \mathrm{~min}$, before being immersed in destaining solution until protein bands were clearly resolved.

Table 6.7. Solutions used for SDS-PAGE analysis

\begin{tabular}{|c|c|}
\hline Solution & Constituents \\
\hline Loading dye & $\begin{array}{c}100 \mathrm{mM} \text { Tris- } \mathrm{HCl}, 20 \%(\mathrm{w} / \mathrm{v}) \text { glycerol, } 4 \%(\mathrm{w} / \mathrm{v}) \mathrm{SDS}, 0.2 \%(\mathrm{w} / \mathrm{v}) \text { bromophenol blue, } 30 \mathrm{mM} \\
\text { 2-mercaptoethanol }\end{array}$ \\
\hline Running buffer & $250 \mathrm{mM}$ Tris- $\mathrm{HCl}, 192 \mathrm{mM}$ glycine, 0.1\% (w/v) SDS \\
\hline Stain & $\begin{array}{l}2.4 \%(w / v) \text { Coomassie Brilliant Blue R-250, } 50 \%(v / v) \text { methanol, } 10 \%(v / v) \text { acetic acid, } 40 \% \\
(v / v) \mathrm{H}_{2} \mathrm{O}\end{array}$ \\
\hline Destain & $50 \%(\mathrm{v} / \mathrm{v}) \mathrm{H}_{2} \mathrm{O}, 40 \%(\mathrm{v} / \mathrm{v})$ methanol, $10 \%(\mathrm{v} / \mathrm{v})$ acetic acid \\
\hline
\end{tabular}




\subsubsection{Protein expression}

An overnight culture was used to inoculate $500 \mathrm{~mL}$ of the growth medium, or $50 \mathrm{~mL}$ for expression trials. Either LB or autoinduction media was used to grow cells. These cultures were incubated at $37^{\circ} \mathrm{C}$ while shaking until the $\mathrm{OD}_{600}$ had reached 0.6 , where upon the compound used to induce expression was added. The inducer type, inducer concentration, the incubation time and incubation temperature were varied for the expression of each malate oxidoreductase. Proteins were expressed using either E. coli strain E. cloni $10 \mathrm{G}$ or E. coli BL21(DE3) Gold. Induced cells were harvested by centrifugation at 4000g for $10 \mathrm{~min}$ and stored at $-20^{\circ} \mathrm{C}$. Successful expression conditions are given in Chapter 2, section 2.2.3.

\subsubsection{Co-expression with molecular chaperones}

Co-expression of SvMDH with chaperone proteins was performed in an attempt to improve its solubility. Co-expression used pGro7 (Takara Bio, Kusatsu, Japan), a commercial plasmid used to express the GroES - GroEL chaperone complex. The pGro7 plasmid allows transformed E. coli to be resistant to chloramphenicol, in addition to the kanamycin resistance conferred by pET-28 a (+). Transformed BL21(DE3) Gold cells containing both plasmids could therefore be selected using LB-agar plates containing both antibiotics.

The protocol used followed the manufacturer's instructions. In brief, co-expression was performed by inoculating $250 \mathrm{~mL}$ LB containing both chloramphenicol and kanamycin. Expression of chaperones was immediately induced by addition of $0.5 \mathrm{mg} / \mathrm{mL}$ L-arabinose, and once the $\mathrm{OD}_{600}$ had reached 0.6, SvMDH expression was induced by $1 \mathrm{mM}$ IPTG. Induced cultures were incubated at $37^{\circ} \mathrm{C}$ for $3 \mathrm{~h}$ while shaking at $200 \mathrm{rpm}$.

\subsubsection{Protein purification}

Harvested cell pellets obtained from sections 6.4.2 and 6.4.3 were resuspended in lysis buffer, which contained $100 \mathrm{mM}$ of either HEPES, potassium phosphate or Tris- $\mathrm{HCl}$, as well as 300 mM NaCl. Lysozyme (Gold Biotechnology, St. Louis, MO, USA) and Benzoase nuclease from Sigma (St Louis, MO, USA) were added to concentrations of $0.5 \mathrm{mg} / \mathrm{mL}$ and $0.5 \mathrm{U} / \mathrm{mL}$, respectively. Per gram of cell pellet, $20 \mu \mathrm{L}$ protease inhibitor cocktail (EDTA-free) (Abcam). 
This cell lysis mixture was incubated for $30 \mathrm{~min}$ at room temperature. Cells were lysed by sonication, using a Vibra-Cel Ultrasonic Liquid Processor, model VCX500 (Sonics and Materials, Newtown, CT, USA). Sonication was performed for $10 \mathrm{~s}$ at a time using $25 \%$ amplitude, with a subsequent $30 \mathrm{~s}$ recovery on ice. This was repeated 10 times for each sample. The soluble protein was separated from the insoluble debris by centrifugation at 20 , $000 \mathrm{~g}$ for $30 \mathrm{~min}$ at $4^{\circ} \mathrm{C}$. The decanted soluble fraction was further clarified by passing it through a filter with a $0.22 \mu \mathrm{m}$ pore size.

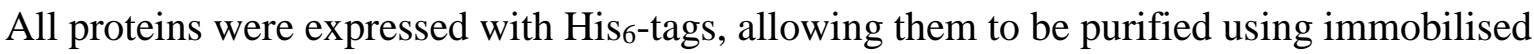
metal affinity chromatography (IMAC). However, two different IMAC protocols were followed.

\subsubsection{Affinity chromatography using Ni-NTA resin and ÄKTA}

Most proteins in this study were purified using either the ÄKTA pure or ÄKTA start (GE Healthcare). Both chromatography systems were used in conjunction with either $1 \mathrm{~mL}$ or 5 mL HisTrap HP columns, which are pre-packed with nickel nitrilotriacetic acid (NTA) resin (GE Healthcare) that binds to His6-tags. A flow rate of $1 \mathrm{~mL} \mathrm{~min}{ }^{-1}$ and $5 \mathrm{~mL} \mathrm{~min}^{-1}$ was used when using $1 \mathrm{~mL}$ and $5 \mathrm{~mL}$ columns, respectively, according to the manufacturer's instructions.

Before applying the soluble fraction, the lines of each ÄKTA system, as well as the column, were first washed and equilibrated, using 10 column volumes for each step. All liquids used by the ÄKTA had been filtered and degassed. The first wash used $20 \%$ ethanol, followed by double-distilled water. The column was further cleaned using lysis buffer containing $500 \mathrm{mM}$ imidazole, eluting any proteins that may be bound to the column from previous runs, followed by an equilibration step that used the lysis buffer without imidazole.

The impure soluble protein sample was loaded on the column using a $50 \mathrm{~mL}$ Superloop (Amersham Biosciences, Amersham, UK) for the ÄKTA pure or the integrated sample loading pump when using the ÄKTA start. Once completely loaded, unbound proteins were washed from the columns using 10 column volumes of lysis buffer, or until the trace of the $280 \mathrm{~nm}$ absorption had reached baseline values. 
The bound protein was eluted from the column using a gradient of imidazole concentrations that ranged from $0-500 \mathrm{mM}$, with $1 \mathrm{~mL}$ fractions of the eluent being collected during this process. Fractions that corresponded to increases in $\mathrm{A}_{280}$ were taken forward for analysis by SDS-PAGE to check for the expected protein size and the purity.

\subsubsection{Affinity chromatography using TALON resin and gravity flow}

Gravity flow was used to purify ApMDH, as well as SvMDH when it was co-expressed with molecular chaperones. The process used the $\mathrm{Co}^{2+}$ - based TALON metal affinity resin (Clontech Laboratories, Mountain View, CA, USA). As per the manufacturer's instructions, 1 $\mathrm{mL}$ of the resin was washed/equilibrated by pelleting the resin by centrifugation for $2 \mathrm{~min}$ at $700 \mathrm{~g}, 4^{\circ} \mathrm{C}$, decanting the supernatant, then resuspending the resin in $5 \mathrm{~mL}$ lysis buffer. This process was repeated twice before the pellet was resuspended in the impure soluble protein sample. The mixture was then incubated at $4^{\circ} \mathrm{C}$ while rocking for $1 \mathrm{~h}$, allowing the His6tagged protein to bind to the resin.

The pelleting and resuspending in lysis buffer procedure was repeated to remove unbound proteins, though the resuspended pellet was left shaking at $4^{\circ} \mathrm{C}$ for $10 \mathrm{~min}$ for each wash. This was also repeated twice, before the resin was transferred into a Poly-Prep chromatography column from Bio-Rad (Hercules, CA, USA). Here, the resin was further washed with $2.5 \mathrm{~mL}$ lysis buffer and $2.5 \mathrm{~mL}$ lysis buffer that contained $10 \mathrm{mM}$ imidazole for the co-expression of SvMDH, these two buffers contained $3 \mathrm{mM} \mathrm{Mg}$-ATP to promote chaperone separation. The bound protein was then eluted as $500 \mu \mathrm{l}$ fractions using lysis buffer $+500 \mathrm{mM}$ imidazole. To test which fractions contained protein, $2 \mu \mathrm{L}$ of each fraction was mixed with $50 \mu 1$ Bradford reagent from Bio-Rad (Hercules, CA, USA). Blue coloured fractions were analysed by SDS-PAGE gel (section 6.4.1).

\subsubsection{Size-exclusion chromatography}

If required, size-exclusion chromatography (SEC) was used to further purify protein samples obtained from affinity chromatography. In conjunction with the ÄKTA pure, the Superdex 75 Increase $10 / 300 \mathrm{GL}$ column was used if the protein sample to be loaded was $500 \mu \mathrm{L}$ or less, 
or the HiLoad 16/600 Superdex 200 pg column for larger sample volumes, up to $5 \mathrm{~mL}$ (both columns from GE Healthcare, Chicago, IL, USA).

Following the manufacturer's instructions, columns used flow rates of $0.5 \mathrm{~mL} \mathrm{~min}^{-1}$ and 1 $\mathrm{mL} \mathrm{min}^{-1}$, respectively. Prior to sample loading, each column was washed with two column volumes of filtered and degassed water, before being equilibrated with the desired storage buffer (e.g. $100 \mathrm{mM}$ HEPES, pH 7). Samples were loaded onto the column by injection loop, though were first centrifuged at $17,000 \mathrm{~g}\left(4^{\circ} \mathrm{C}, 5 \mathrm{~min}\right)$ and filtered through a $0.22 \mu \mathrm{m}$ pore size syringe driven filter to remove any insoluble particles. As for affinity chromatography, fractions corresponding to increases in $\mathrm{A}_{280}$ were collected and analysed by SDS-PAGE (section 6.4.1).

\subsubsection{Buffer exchange, concentrating, quantification and storage of purified proteins}

Fractions containing purified proteins were pooled and concentrated using a $10 \mathrm{kDa}$ Amicon Ultra-15 Centrifugal Filter Unit from MilliporeSigma (Burlington, MA, USA) and centrifugation at $2,800 \mathrm{~g}, 4^{\circ} \mathrm{C}$. The protocol for buffer exchange also used these filters, with pooled fractions being concentrated to approximately $200 \mu \mathrm{L}$ before being re-diluted to 15 $\mathrm{mL}$ in the desired buffer. This process was repeated twice, before re-concentrating the sample back to $200 \mu \mathrm{L}$. Split into $10 \mu \mathrm{L}$ aliquots, the now purified and concentrated protein samples were stored within $1.5 \mathrm{~mL}$ microcentrifuge tubes at $-80^{\circ} \mathrm{C}$ for long-term storage, or $4^{\circ} \mathrm{C}$ for the short-term $(<24 \mathrm{~h})$.

The protein concentration was measured using a Cary $100 \mathrm{UV}-\mathrm{V}$ is spectrophotometer and 80 $\mu \mathrm{L}$ quartz cuvette (both from Agilent, Santa Clara, CA, USA). Absorbance over wavelengths of $200-300 \mathrm{~nm}$ were measured using the spectrophotometer's scan function, with the reading at $280 \mathrm{~nm}$ being used to calculate protein concentration as given by the Beer-Lambert law: 


$$
\mathrm{c}=\frac{\mathrm{A}}{\varepsilon \times \mathrm{l}}
$$

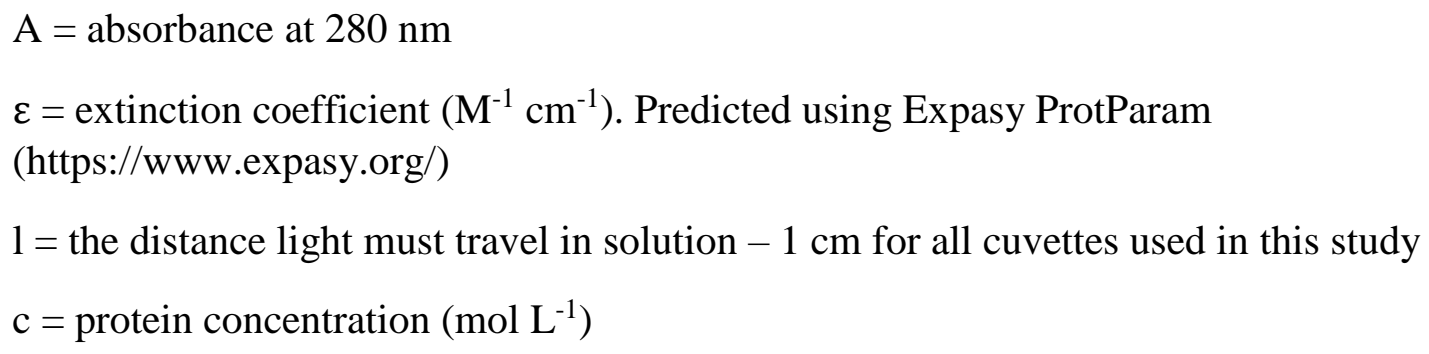

As protein samples often had to be diluted for the absorbance to fall within the operational range of the spectrophotometer, the calculated concentrations accounted for the dilution factor. For each $\mathrm{A}_{280}$ measurement, a background scan was performed, without protein, with the difference being used for concentration calculations. Concentration measurements were performed in triplicate, with the average being taken as the final value.

\subsection{Kinetic characterisation of malate oxidoreductases}

To help select a lead candidate, spectrophotometric assays were used to investigate kinetic behaviour of chosen malate oxidoreductases. Assays were performed using $1.4 \mathrm{~mL}$ quartz cuvettes (Hellma Analytics), with measurements made by Cary $100 \mathrm{UV}-\mathrm{Vis}$ spectrophotometer and the temperature maintained by an accessory temperature controller module (both from Agilent, Santa Clara, CA, USA). The increase in A340 was measured, corresponding to the production of $\mathrm{NAD}(\mathrm{P}) \mathrm{H}$.

The reagents used in assays varied between malate oxidoreductases. All assays were equilibrated to the set temperature for $5 \mathrm{~min}$, and initial rates were measured as the gradient of the linear portion of kinetics traces, typically manifesting as the rate observed in the first 30 seconds of each assay. For Michaelis-Menten kinetics, the concentration of one compound was varied, with all others fixed at saturating concentrations, between 5 to $10 \times K_{\mathrm{M}}$. Where saturating kinetics could not be observed for a compound, such as for AsME(R181Q) when varying malate or $\mathrm{Mg}^{2+}$, concentrations were fixed at the highest possible, with calculated parameters being labelled as "apparent". The concentrations tested for the varied compound 
were sequentially increased from zero with the aim of the plotted initial rates producing a hyperbolic curve, showing clear saturation kinetics. Each concentration was measured in triplicate, though replicates were performed in a random order to ensure that changes in initial rate were not a product of enzyme activity loss or the degradation of reagents. Specific assay conditions for each enzyme are given in Table 6.8 .

Table 6.8. Assay conditions of each candidate malate oxidoreductase

\begin{tabular}{|c|c|}
\hline Enzyme & Conditions used \\
\hline AsME & $\begin{array}{l}1-5 \mathrm{mM} \mathrm{NAD}^{+}, 25-200 \mathrm{mM} \mathrm{MgCl}_{2}, 0-300 \mathrm{mM} \mathrm{NH}_{4} \mathrm{Cl}, 100 \mathrm{mM} \mathrm{HEPES} \text { buffer } \mathrm{pH} 7,25^{\circ} \mathrm{C} \text {. Reaction started by } \\
\text { addition of malate. }\end{array}$ \\
\hline AcME & $\begin{array}{l}1.5 \mathrm{mM} \mathrm{NAD}^{+}, 3 \mathrm{mM} \mathrm{MgCl}_{2}, 5 \mathrm{mM} \mathrm{NH}_{4} \mathrm{Cl}, 100 \mu \mathrm{M} \mathrm{CoA}, 100 \mathrm{mM} \text { HEPES buffer } \mathrm{pH} 7,25^{\circ} \mathrm{C} \text {. Reaction started by } \\
\text { addition of enzyme. }\end{array}$ \\
\hline ApMDH & 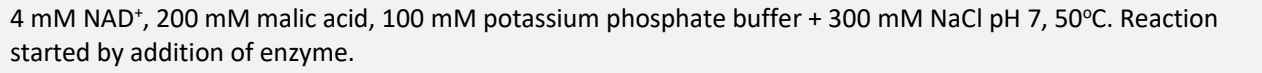 \\
\hline MaeA & 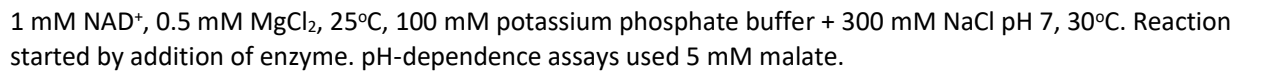 \\
\hline TmME & $\begin{array}{l}1 \mathrm{mM} \mathrm{NAD}(\mathrm{P})^{+}, 0.5 \mathrm{mM} \mathrm{MgCl} 2,100 \mathrm{mM} \text { potassium phosphate buffer }+300 \mathrm{mM} \mathrm{NaCl} \mathrm{pH} 7,25-50^{\circ} \mathrm{C} \text {. Reaction } \\
\text { started by addition of enzyme. }\end{array}$ \\
\hline
\end{tabular}

Initial rates were converted to velocity $\left(\mathrm{s}^{-1}\right)$ using the molar extinction coefficient for NAD(P)H: 6,220 M-1 $\mathrm{cm}^{-1}$ (Veskoukis et al., 2018), and the concentration of the enzyme. Plots were fitted with the Michaelis-Menten equation using GraphPad Prism, allowing both $K_{\mathrm{M}}$ and $k_{\text {cat }}$ to be calculated:

$$
V=\frac{k_{\mathrm{cat}}[\mathrm{S}]}{K_{\mathrm{M}}+[\mathrm{S}]}
$$

Certain plots were fitted to the Haldane model for substrate inhibition by using the following equation (Copeland, 2000): 


$$
V=\frac{k_{\mathrm{cat}}[\mathrm{S}]}{K_{\mathrm{M}}+[\mathrm{S}]+\frac{[\mathrm{S}]^{2}}{K_{\mathrm{i}}}}
$$

For both equations:

$V=$ enzyme velocity $\left(\mathrm{s}^{-1}\right)$

$K_{\text {cat }}=$ turnover number $\left(\mathrm{s}^{-1}\right)$

$K_{\mathrm{M}}=$ the concentration required for half the maximum velocity

$K_{\mathrm{i}}=$ the inhibition constant

$\mathrm{S}=$ substrate concentration

\section{6. $\mathrm{pH}$-dependence assays}

Studies into the effect of $\mathrm{pH}$ on enzyme activity used the spectrophotometric assays described in section 6.5. Activity was measured over a $\mathrm{pH}$ range of $3-10$, initially using a series of different buffers as the effective buffering range of any one buffer type is typically limited to $\pm 1 \mathrm{pH}$ unit from the $\mathrm{p} K_{\mathrm{a}}$. A list of these buffers is shown in Table 6.9.

Table 6.9. Buffers used to investigate the $\mathrm{pH}$ dependence of candidate malate oxidoreductases. Each solution contained $100 \mathrm{mM}$ of the buffering molecule and was brought to the desired $\mathrm{pH}$ using concentrated $\mathrm{NaOH}$ and $\mathrm{HCl}$.

\begin{tabular}{cc} 
Buffer & pH range used \\
\hline Citrate & $3-6$ \\
MES & $5.5-6.5$ \\
Potassium Phosphate & $6-8$ \\
Tris- $\mathrm{HCl}$ & $7.5-9$ \\
Glycine & $8.5-10.5$ \\
\hline
\end{tabular}


Overlapping $\mathrm{pH}$ ranges were chosen to compare the effect of the type of buffer on activity. As certain assays used high concentrations of malic acid (up to $100 \mathrm{mM}$ ), whose acidity may overcome the buffering capacity and undesirably decrease the $\mathrm{pH}$, buffers were mixed with malic acid prior to being brought to the correct $\mathrm{pH}$ using concentrated $\mathrm{NaOH}(5 \mathrm{M})$ and $\mathrm{HCl}$ (12 M). After observing that certain buffers appear to inhibit enzyme activity, the assays switched to using potassium phosphate buffer only, for the entire $\mathrm{pH}$ range tested.

\subsection{Electrochemical techniques}

All electrochemical experiments used C220BT screen-printed electrodes from Metrohm Dropsens (Asturias, Spain). Each electrode featured a planar gold working electrode with an area of $0.13 \mathrm{~cm}^{2}$ (4 mm diameter), a gold counter electrode and silver reference electrode. Electrochemical measurements were performed using the Digi-Ivy (Austin, TX, USA) DY2116B potentiostat, with the subsequent analysis being performed with the integrated software. Assays were performed at room temperature, and used $50 \mu \mathrm{L}$ of the testing solution, which was sufficient to fully cover all three electrodes. All currents are reported using the IUPAC convention, which defines oxidation currents (electrons are transferred to the electrode, also known as anodic current) as positive.

\subsubsection{Preparation of electrodes}

Gold is particularly susceptible to fouling by ambient contaminants (Fischer et al., 2009). As electrochemistry experiments were not performed in a clean-room, electrodes were cleaned each day before use by immersion in a $1 \%$ solution of Decon 90 surfactant, then sonication using the VCX 500 Vibra-Cel sonicator (Sonics and Materials, Newtown, CT, USA), 20\% amplitude for $5 \mathrm{~min}$. After rinsing with copious amounts of double-distilled water, electrodes were dried by a directed stream of air. Electrodes were re-used after being washed with $70 \%$ ethanol and water, though those with visible scratches or fouling that could not be removed by sonication were immediately discarded. 


\subsubsection{Cyclic voltammetry}

Cyclic voltammetry was used to characterise the electron mediators that were considered for use in the biosensor. Each mediator ( $5 \mathrm{mM}$, final concentration) was dissolved in $100 \mathrm{mM}$ HEPES buffer $\mathrm{pH} 7$ containing $200 \mathrm{mM} \mathrm{MgCl}_{2}$ and $300 \mathrm{mM} \mathrm{NH}_{4} \mathrm{Cl}$. When $\mathrm{NAD}^{+}$was used, $5 \mathrm{mM}$ was added to the buffer. Voltammograms were produced by sweeping the potential from +500 to $-500 \mathrm{mV}$, and back, at a scan rate of $100 \mathrm{mV} / \mathrm{s}$ for one cycle. Peak oxidation currents and peak oxidation potentials were calculated by the potentiostat's integrated software, with the latter being determined by extrapolating the baseline, which was performed automatically by the program.

\subsubsection{Chronoamperometry}

Chronoamperometry experiments used $100 \mathrm{mM}$ HEPES pH 7², $200 \mathrm{mM}$ divalent metal ion (either $\mathrm{Mg}^{2+}$ or $\mathrm{Mn}^{2+}$ ) $1,300 \mathrm{mM} \mathrm{NH} \mathrm{Cl}^{1}, 10 \mathrm{mM} \mathrm{NAD}^{+2}, 10.2 \mu \mathrm{M} \mathrm{AsME}^{3}$ and $0.5 \mathrm{mM}$ 2,6dichloroindophenol ${ }^{4}$. Certain assays also contained $100 \mathrm{mM}$ citrate $^{1}$. Together, these compounds were unstable as a master mix solution, as shown by a decrease in current response. Instead, compounds were added sequentially, directly onto the electrode in the order marked by the superscript after each reagent. 2,6-dichloroindophenol was particularly unstable in solution containing HEPES, therefore measurements were made within two min of it being added.

When using the finalised biosensor architecture, reactions were started by the addition of malate or grape juice/wine sample and were allowed to proceed for two min. Current measurements were taken after a five second pulse at $+150 \mathrm{mV}$, with the sensitivity $\left(\mathrm{A} \mathrm{V}^{-1}\right)$ setting of the potentiostat being modified for the expected size of the current. As the majority of current readings were between $1-10 \mu \mathrm{A}$, the potentiostat's $10 \mu \mathrm{A} \mathrm{V}^{-1}$ sensitivity setting was used for most measurements. Recorded currents were standardised for the electrode area, and were therefore reported as current density. 


\subsection{Preparation of real samples}

Grape juice and wine samples were sourced from either the Hare's Run vineyard in the Wairarapa region of New Zealand, or from Mount Difficulty Wines in Central Otago, also in New Zealand. Pinot Gris and Pinot Noir samples were taken at various points during winemaking process, including grapes from the vine, directly post-harvest and during alcoholic fermentation. Such specifications have been described alongside the corresponding results in each chapter.

Grape samples were blended for 5 min using the Mix and Go food processor (George Foreman) before being applied to the biosensor. Certain samples were decolourised using activated carbon: first, grape juice samples were centrifuged at $4000 \mathrm{~g}, 5 \mathrm{~min}, 25^{\circ} \mathrm{C}$ to remove insoluble grape matter. The soluble fraction was then filtered through muslin cloth before adding $25 \mathrm{mg}$ activated carbon per $\mathrm{mL}$ of grape juice. The mixture was then incubated at room temperature while rocking for $5 \mathrm{~min}$. The activated carbon was then removed by repeating the centrifugation step and separating the soluble and insoluble fractions. When not used, real samples, processed or unprocessed, were stored at $-20^{\circ} \mathrm{C}$. 


\section{Chapter Seven - References}

Andersen, R. A. and Sowers, J. A. (1968) 'Optimum conditions for bonding of plant phenols to insoluble polyvinylpyrrolidone', Phytochemistry, 7(2), pp. 293-301

Anderson, K., Nelgen, S. and Pinilla, V. (2017) Global Wine Markets, 1860 to 2016: A Statistical Compendium. Adelaide: University of Adelaide Press

Antonelli, M. L., Spadaro, C. and Tornelli, R. F. (2008) 'A microcalorimetric sensor for food and cosmetic analyses: L-malic acid determination', Talanta, 74(5), pp. 1450-1454

Arif, M., Setford, S. J., Burton, K. S. and Tothill, I. E. (2002) 'L-Malic acid biosensor for field-based evaluation of apple, potato and tomato horticultural produce', Analyst, 127(1), pp. 104-108

Arvinte, A., Rotariu, L. and Bala, C. (2008) 'Amperometric low-potential detection of malic acid using single-wall carbon nanotubes based electrodes', Sensors, 8(3), pp. 14971507

Arvinte, A., Rotariu, L., Bala, C. and Gurban, A. M. (2009) 'Synergistic effect of mediatorcarbon nanotube composites for dehydrogenases and peroxidases based biosensors', Bioelectrochemistry, 76(1), pp. 107-114

Asensi-Fabado, M. A. and Munné-Bosch, S. (2010) 'Vitamins in plants: occurrence, biosynthesis and antioxidant function', Trends in Plant Science, 15(10), pp. 582-592

Bard, A. L. (2001) Electrochemical methods fundamentals and applications. New York: John Wiley \& Sons.

Bayda, S., Adeel, M., Tuccinardi, T., Cordani, M. and Rizzolio, F. (2019) 'The history of nanoscience and nanotechnology: from chemical-physical applications to nanomedicine', Molecules, 25(1), pp. 112

Baynes, J. and Dominiczak, M. H. (2009) Medical biochemistry. Elsevier Health Sciences.

Bergmeyer, H. U. I. (1975) Methods of Enzymatic Analysis, Volume 2. New York: Academic Press.

Bernstein, L. H. and Everse, J. (1978) 'Studies on the mechanism of the malate dehydrogenase reaction', Journal of Biological Chemistry, 253(24), pp. 8702-8707

Bhalla, N., Jolly, P., Formisano, N. and Estrela, P. (2016) 'Introduction to biosensors', Essays in Biochemistry, 60(1), pp. 1-8

Bisswanger, H. (2014) 'Enzyme assays', Perspectives in Science, 1(1), pp. 41-55

Blaedel, W. J. and Engstrom, R. C. (1980) 'Reagentless enzyme electrodes for ethanol, lactate, and malate', Analytical Chemistry, 52(11), pp. 1691-1697 
Blandón-Naranjo, L., Della Pelle, F., Vázquez, M. V., Gallego, J., Santamaría, A., AlzateTobón, M. and Compagnone, D. (2018) 'Electrochemical Behaviour of Microwaveassisted Oxidized MWCNTs Based Disposable Electrodes: Proposal of a NADH Electrochemical Sensor', Electroanalysis, 30(3), pp. 509-516

Bologna, F. P., Andreo, C. S. and Drincovich, M. F. (2007) 'Escherichia coli malic enzymes: two isoforms with substantial differences in kinetic properties, metabolic regulation, and structure', Journal of bacteriology, 189(16), pp. 5937-5946

Bononi, M., Quaglia, G. and Tateo, F. (2016) 'Preliminary LC-IRMS characterization of Italian pure lemon juices and evaluation of commercial juices distributed in the Italian market', Food Anal. Methods, 9(10), pp. 2824-2831

Borgmann, S., Schulte, A., Neugebauer, S. and Schuhmann, W. (2012) 'Amperometric biosensors', Advances in Electrochemical Sciences and Engineering, 13, pp. 1-83

Borsook, H. and Keighley, G. (1933) 'Oxidation-reduction potential of ascorbic acid (vitamin C)', Proceedings of the National Academy of Sciences of the United States of America, 19(9), pp. 875-878

Bucur, B., Mallat, E., Gurban, A.-M., Gocheva, Y., Velasco, C., Marty, J.-L. and Noguer, T. (2006) 'Strategies to develop malic acid biosensors based on malate quinone oxidoreductase (MQO)', Biosensors and Bioelectronics, 21(12), pp. 2290-2297

Caro-Jara, N., Mundaca-Uribe, R., Zaror-Zaror, C., Carpinelli-Pavisic, J., Aranda-Bustos, M. and Peña-Farfal, C. (2013) 'Development of a Bienzymatic Amperometric Glucose Biosensor Using Mesoporous Silica (MCM-41) for Enzyme Immobilization and Its Application on Liquid Pharmaceutical Formulations', Electroanalysis, 25(1), pp. 308315

Casati, P., Spampinato, C. P. and Andreo, C. S. (1997) 'Characteristics and Physiological Function of NADP-Malic Enzyme from Wheat', Plant and Cell Physiology, 38(8), pp. 928-934

Chang, A., Jeske, L., Ulbrich, S., Hofmann, J., Koblitz, J., Schomburg, I., Neumann-Schaal, M., Jahn, D. and Schomburg, D. (2020) 'BRENDA, the ELIXIR core data resource in 2021: new developments and updates', Nucleic Acids Research, 49(D1), pp. D498D508

Chang, G.-G. and Tong, L. (2003) 'Structure and function of malic enzymes, a new class of oxidative decarboxylases', Biochemistry, 42(44), pp. 12721-12733

Chiorcea-Paquim, A.-M., Enache, T. A., De Souza Gil, E. and Oliveira-Brett, A. M. (2020) 'Natural phenolic antioxidants electrochemistry: towards a new food science methodology', Comprehensive Reviews in Food Science and Food Safety, 19(4), pp. $1680-1726$

Cho, J.-H., Yang, J.-W., Yabuki, S. and Mizutani, F. (1995) 'Construction of carbon paste enzyme electrodes for the measurement of L-malate with polyethylene glycolmodified malate dehydrogenase', Denki Kagaku oyobi Kogyo Butsuri Kagaku, 63(12), pp. $1143-1144$ 
Clark, L. C., Jr. and Lyons, C. (1962) 'Electrode systems for continuous monitoring in cardiovascular surgery', Annals of the New York Academy of Sciences, 102, pp. 29-45

Clausen, M. R., Pedersen, B. H., Bertram, H. C. and Kidmose, U. (2011) 'Quality of sour cherry juice of different clones and cultivars (Prunus cerasus L.) determined by a combined sensory and NMR spectroscopic approach', Journal of Agricultural and Food Chemistry, 59(22), pp. 12124-12130

Coleman, D. E., Rao, G. S. J., Goldsmith, E. J., Cook, P. F. and Harris, B. G. (2002) 'Crystal Structure of the Malic Enzyme from Ascaris suum Complexed with Nicotinamide Adenine Dinucleotide at 2.3 Å Resolution', Biochemistry, 41(22), pp. 6928-6938

Copeland, R. A. (2000) Enzymes: a practical introduction to structure, mechanism, and data analysis. John Wiley \& Sons.

Cordova, A. C. and Sumpio, B. E. (2009) 'Polyphenols are medicine: is it time to prescribe red wine for our patients?', International Journal of Angiology, 18(3), pp. 111-117

Cottrell, F. G. (1903) 'Der Reststrom bei galvanischer Polarisation, betrachtet als ein Diffusionsproblem', Zeitschrift für Physikalische Chemie, 42U(1), pp. 385-431

Crétin, C., Luchetta, P., Joly, C., Decottignies, P., Lepiniec, L., Gadal, P., Sallantin, M., Huet, J. C. and Pernollet, J. C. (1990) 'Primary structure of sorghum malate dehydrogenase (NADP) deduced from cDNA sequence. Homology with malate dehydrogenase (NAD)', European Journal of Biochemistry, 192(2), pp. 299-303

Cunningham, M. A., Ho, L. L., Nguyen, D. T., Gillilan, R. E. and Bash, P. A. (1997) 'Simulation of the enzyme reaction mechanism of malate dehydrogenase', Biochemistry, 36(16), pp. 4800-4816

Dasenaki, M. E. and Thomaidis, N. S. (2019) 'Quality and authenticity control of fruit juices - a review', Molecules, 24(6), pp. 1014

Datta, S., Christena, L. R. and Rajaram, Y. R. S. (2013) 'Enzyme immobilization: an overview on techniques and support materials', 3 Biotech, 3(1), pp. 1-9

Derradji-Benmeziane, F., Djamai, R. and Cadot, Y. (2014) 'Antioxidant capacity, total phenolic, carotenoid, and vitamin $\mathrm{C}$ contents of five table grape varieties from Algeria and their correlations', OENO One, 48(2), pp. 153-162

Ehling, S. and Cole, S. (2011) 'Analysis of organic acids in fruit juices by liquid chromatography-mass spectrometry: an enhanced tool for authenticity testing', Journal of Agricultural and Food Chemistry, 59(6), pp. 2229-2234

Elgrishi, N., Rountree, K. J., McCarthy, B. D., Rountree, E. S., Eisenhart, T. T. and Dempsey, J. L. (2018) 'A Practical Beginner's Guide to Cyclic Voltammetry', Journal of Chemical Education, 95(2), pp. 197-206

Englard, S. and Siegel, L. (1969) 'Mitochondrial L-malate dehydrogenase of beef heart [EC 1.1.1.37 L-Malate: NAD oxidoreductase]', Methods in Enzymology, 13, pp. 99-106 
Esti, M., Volpe, G., Micheli, L., Delibato, E., Compagnone, D., Moscone, D. and Palleschi, G. (2004) 'Electrochemical biosensors for monitoring malolactic fermentation in red wine using two strains of Oenococcus oeni', Analytica Chimica Acta, 513(1), pp. 357364

Eyduran, S. P., Akin, M., Ercisli, S., Eyduran, E. and Maghradze, D. (2015) 'Sugars, organic acids, and phenolic compounds of ancient grape cultivars (Vitis vinifera L.) from Igdir province of Eastern Turkey', Biological research, 48(1), pp. 2-2

Fischer, L. M., Tenje, M., Heiskanen, A. R., Masuda, N., Castillo, J., Bentien, A., Émneus, J., Jakobsen, M. H. and Boisen, A. (2009) 'Gold cleaning methods for electrochemical detection applications', Microelectronic Engineering, 86(4), pp. 1282-1285

Francis, D. M. and Page, R. (2010) 'Strategies to optimize protein expression in E. coli', Current protocols in protein science, Chapter 5(1), pp. 5241-52429

Gajovic, N., Warsinke, A. and Scheller, F. W. (1997) 'Comparison of two enzyme sequences for a novel L-malate biosensor', Journal of Chemical Technology \& Biotechnology, 68(1), pp. 31-36

Gajovic, N., Warsinke, A. and Scheller, F. W. (1998) 'A bienzyme electrode for L-malate based on a novel and general design', Journal of Biotechnology, 61(2), pp. 129-133

Gamella, M., Campuzano, S., Conzuelo, F., Curiel, J. A., Muñoz, R., Reviejo, A. J. and Pingarrón, J. M. (2010) 'Integrated multienzyme electrochemical biosensors for monitoring malolactic fermentation in wines', Talanta, 81(3), pp. 925-933

Ganesh, I., Ravikumar, S., Park, S. J., Lee, S. H. and Hong, S. H. (2013) 'Expression characteristics of the maeA and maeB genes by extracellular malate and pyruvate in Escherichia coli', Korean Journal of Chemical Engineering, 30(7), pp. 1443-1447

Ganesh, I., Ravikumar, S., Yoo, I. K. and Hong, S. H. (2015) 'Construction of malate-sensing Escherichia coli by introduction of a novel chimeric two-component system', Bioprocess and Biosystems Engineering, 38(4), pp. 797-804

García-Pérez, P., Losada-Barreiro, S., Gallego, P. P. and Bravo-Díaz, C. (2019) 'Adsorption of gallic acid, propyl gallate and polyphenols from Bryophyllum extracts on activated carbon', Scientific Reports, 9(1), pp. 14830

Ge, Y. D., Cao, Z. Y., Wang, Z. D., Chen, L. L., Zhu, Y. M. and Zhu, G. P. (2010) 'Identification and biochemical characterization of a thermostable malate dehydrogenase from the mesophile Streptomyces coelicolor A3(2)', Bioscience, Biotechnology, and Biochemistry, 74(11), pp. 2194-2201

Gharib, G., Rashid, N., Bashir, Q., Gardner, Q. T., Akhtar, M. and Imanaka, T. (2016) 'Pcal_1699, an extremely thermostable malate dehydrogenase from hyperthermophilic archaeon Pyrobaculum calidifontis', Extremophiles, 20(1), pp. 57-67

Gibson, T. D. (1999) 'Biosensors: the stabilité problem', Analusis, 27(7), pp. 630-638 
Gil, D. M. A. and Rebelo, M. J. F. (2011) 'Gallic acid interference on polyphenolic amperometric biosensing using Trametes versicolor laccase', Journal of Molecular Catalysis B: Enzymatic, 72(3), pp. 193-198

Gil, J. V., Mateo, J. J., Jiménez, M., Pastor, A. and Huerta, T. (1996) 'Aroma Compounds in Wine as Influenced by Apiculate Yeasts', Journal of Food Science, 61(6), pp. 12471250

Giménez-Gómez, P., Gutiérrez-Capitán, M., Capdevila, F., Puig-Pujol, A., FernándezSánchez, C. and Jiménez-Jorquera, C. (2017) 'Robust L-malate bienzymatic biosensor to enable the on-site monitoring of malolactic fermentation of red wines', Analytica Chimica Acta, 954, pp. 105-113

Girelli, A. M., Mattei, E. and Messina, A. (2006) 'Phenols removal by immobilized tyrosinase reactor in on-line high performance liquid chromatography', Analytica Chimica Acta, 580(2), pp. 271-277

Golovanov, A. P., Hautbergue, G. M., Wilson, S. A. and Lian, L. Y. (2004) 'A Simple Method for Improving Protein Solubility and Long-Term Stability', Journal of the American Chemical Society, 126(29), pp. 8933-8939

Good, N. E., Winget, G. D., Winter, W., Connolly, T. N., Izawa, S. and Singh, R. M. M. (1966) 'Hydrogen Ion Buffers for Biological Research', Biochemistry, 5(2), pp. 467477

Goward, C. R. and Nicholls, D. J. (1994) 'Malate dehydrogenase: a model for structure, evolution, and catalysis', Protein Science, 3(10), pp. 1883-1888

Grieshaber, D., MacKenzie, R., Vörös, J. and Reimhult, E. (2008) 'Electrochemical biosensors - sensor principles and architectures', Sensors, 8(3), pp. 1400-1458

Grimm, F. C. and Doherty, D. G. (1961) 'Properties of the Two Forms of Malic Dehydrogenase from Beef Heart', Journal of Biological Chemistry, 236(7), pp. 19801985

Grissom, C. B., Canellas, P. F. and Wedding, R. T. (1983) 'Allosteric regulation of the NAD malic enzyme from cauliflower: activation by fumarate and coenzyme A', Archives of Biochemistry and Biophysics, 220(1), pp. 133-144

Gurban, A. M., Prieto-Simón, B., Marty, J. L. and Noguer, T. (2006) 'Malate biosensors for the monitoring of malolactic fermentation: different approaches', Analytical Letters, 39(8), pp. 1543-1558

Gutiérrez-Gamboa, G., Garde-Cerdán, T., Moreno-Simunovic, Y. and Pérez-Álvarez, E. P. (2019) '10 - Amino Acid Composition of Grape Juice and Wine: Principal Factors That Determine Its Content and Contribution to the Human Diet', in Grumezescu, A.M. \& Holban, A.M. (eds.) Nutrients in Beverages: Academic Press, pp. 369-391.

Hayat, A., Catanante, G. and Marty, J. L. (2014) 'Current trends in nanomaterial-based amperometric biosensors', Sensors, 14(12), pp. 23439-23461 
Hellinga, H. W. and Marvin, J. S. (1998) 'Protein engineering and the development of generic biosensors', Trends in Biotechnology, 16(4), pp. 183-189

Hemraj, S., Hari Prasad, S., Ashish, K., Omraj, D. and Rupa, G. (2019) 'A comparative anaylsis of vitamin $\mathrm{C}$ concentration in commercial fruit juices and fresh fruits of Nepal with effect of temperature', International Journal of Pharmacy and Pharmaceutical Sciences, 11(8), pp. 46-51

Holaday, A. S. and Gary, W. L. (1989) 'Effect of pH on the Kinetic Parameters of NADPMalic Enzyme from a C 4 Flaveria (Asteraceae) Species', Plant Physiology, 90(2), pp. 401-405

Huber, R., Langworthy, T. A., König, H., Thomm, M., Woese, C. R., Sleytr, U. B. and Stetter, K. O. (1986) 'Thermotoga maritima sp. nov. represents a new genus of unique extremely thermophilic eubacteria growing up to $90^{\circ} \mathrm{C}^{\prime}$, Archives of Microbiology, 144(4), pp. 324-333

Iijima, S., Saiki, T. and Beppu, T. (1980) 'Physicochemical and catalytic properties of thermostable malate dehydrogenase from an extreme thermophile Thermus flavus AT62', Biochimica et Biophysica Acta - Enzymology, 613(1), pp. 1-9

Iland, P., Bruer, N., Edwards, G., Caloghiris, S. and Wilkes, E. (2013) Chemical Analysis of Grapes and Wine: Techniques and Concepts (2nd ed.). Adelaide: Patrick Iland Wine Promotions Pty Ltd.

Iritani, N., Ikeda, Y., Fukuda, H. and Katsurada, A. (1984) 'Comparative study of lipogenic enzymes in several vertebrates', Lipids, 19(11), pp. 828-835

Jahn, B., Jonasson, N. S. W., Hu, H., Singer, H., Pol, A., Good, N. M., den Camp, H. J. M. O., Martinez-Gomez, N. C. and Daumann, L. J. (2020) 'Understanding the chemistry of the artificial electron acceptors PES, PMS, DCPIP and Wurster's Blue in methanol dehydrogenase assays', JBIC Journal of Biological Inorganic Chemistry, 25(2), pp. 199-212

Jernejc, K. and Legiša, M. (2002) 'The influence of metal ions on malic enzyme activity and lipid synthesis in Aspergillus niger', FEMS Microbiology Letters, 217(2), pp. 185-190

Johnson, A. H. and Stokes, J. L. (1966) 'Manganese Oxidation by Sphaerotilus discophorus', Journal of Bacteriology, 91(4), pp. 1543-1547

Kambourova, M. (2018) 'Thermostable enzymes and polysaccharides produced by thermophilic bacteria isolated from Bulgarian hot springs', Engineering in Life Sciences, 18(11), pp. 758-767

Kanellis, A. K. and Roubelakis - Angelakis, K. A. (1993) 'Grape', in Seymour, G.B., Taylor, J.E. \& Tucker, G.A. (eds.) Biochemistry of Fruit Ripening. Dordrecht: Springer Netherlands, pp. 189-234.

Kanyong, P., Krampa, F. D., Aniweh, Y. and Awandare, G. A. (2017) 'Enzyme-based amperometric galactose biosensors: a review', Microchimica Acta, 184(10), pp. 36633671 
Karsten, W. E. and Cook, P. F. (2007) 'Multiple roles of arginine 181 in binding and catalysis in the NAD-malic enzyme from Ascaris suum', Biochemistry, 46(50), pp. 1457814588

Karsten, W. E., Pais, J. E., Rao, G. S., Harris, B. G. and Cook, P. F. (2003) 'Ascaris suum NAD-malic enzyme is activated by L-malate and fumarate binding to separate allosteric sites', Biochemistry, 42(32), pp. 9712-9721

Kather, B., Stingl, K., van der Rest, M. E., Altendorf, K. and Molenaar, D. (2000) 'Another unusual type of citric acid cycle enzyme in Helicobacter pylori: the malate:quinone oxidoreductase', Journal of Bacteriology, 182(11), pp. 3204-3209

Katrlík, J., Pizzariello, A., Mastihuba, V. r., Švorc, J., Stred'anský, M. and Miertuš, S. (1999) 'Biosensors for L-malate and L-lactate based on solid binding matrix', Analytica Chimica Acta, 379(1), pp. 193-200

Kawakami, R., Sakuraba, H., Goda, S., Tsuge, H. and Ohshima, T. (2009) 'Refolding, characterization and crystal structure of (S)-malate dehydrogenase from the hyperthermophilic archaeon Aeropyrum pernix', Biochimica et Biophysica Acta, 1794(10), pp. 1496-504

Kelly, C. A., Nishiyama, M., Ohnishi, Y., Beppu, T. and Birktoft, J. J. (1993) 'Determinants of protein thermostability observed in the $1.9-\AA$ crystal structure of malate dehydrogenase from the thermophilic bacterium Thermus flavus', Biochemistry, 32(15), pp. 3913-3922

Kitagawa, M., Ara, T., Arifuzzaman, M., Ioka-Nakamichi, T., Inamoto, E., Toyonaga, H. and Mori, H. (2005) 'Complete set of ORF clones of Escherichia coli ASKA library (A Complete Set of E. coli K -12 ORF Archive): Unique Resources for Biological Research', DNA Research, 12(5), pp. 291-299

Kliewer, W. M., Howarth, L. and Omori, M. (1967) 'Concentrations of Tartaric Acid and Malic Acids and Their Salts in Vitis Vinifera Grapes', American Journal of Enology and Viticulture, 18(1), pp. 42-54

Knape, M. J., Ballez, M., Burghardt, N. C., Zimmermann, B., Bertinetti, D., Kornev, A. P. and Herberg, F. W. (2017) 'Divalent metal ions control activity and inhibition of protein kinases', Metallomics, 9(11), pp. 1576-1584

Kövilein, A., Kubisch, C., Cai, L. and Ochsenreither, K. (2020) 'Malic acid production from renewables: a review', Journal of Chemical Technology \& Biotechnology, 95(3), pp. $513-526$

Kretzschmar, U., Rückert, A., Jeoung, J. H. and Görisch, H. (2002) 'Malate:quinone oxidoreductase is essential for growth on ethanol or acetate in Pseudomonas aeruginosa', Microbiology (Reading), 148(Pt 12), pp. 3839-3847

Kucherenko, I. S., Soldatkin, O. O., Dzyadevych, S. V. and Soldatkin, A. P. (2020) 'Electrochemical biosensors based on multienzyme systems: main groups, advantages and limitations - a review', Analytica Chimica Acta, 1111, pp. 114-131 
Kulkarni, G., Cook, P. F. and Harris, B. G. (1993) 'Cloning and Nucleotide Sequence of a Full-length cDNA Encoding Ascaris suum Malic Enzyme', Archives of Biochemistry and Biophysics, 300(1), pp. 231-237

Kwon, Y. D., Kwon, O. H., Lee, H. S. and Kim, P. (2007) 'The effect of NADP-dependent malic enzyme expression and anaerobic C4 metabolism in Escherichia coli compared with other anaplerotic enzymes', Journal of Applied Microbiology, 103(6), pp. 23402345

Landsperger, W. J. and Harris, B. G. (1976) 'NAD+-malic enzyme. Regulatory properties of the enzyme from Ascaris suum', Journal of Biological Chemistry, 251(12), pp. 35993602

Lasik-Kurdyś, M., Majcher, M. and Nowak, J. (2018) 'Effects of Different Techniques of Malolactic Fermentation Induction on Diacetyl Metabolism and Biosynthesis of Selected Aromatic Esters in Cool-Climate Grape Wines', Molecules (Basel, Switzerland), 23(10), pp. 2549

Lemaire, M., Miginiac-Maslow, M. and Decottignies, P. (1996) 'The catalytic site of chloroplastic NADP-dependent malate dehydrogenase contains a His/Asp pair', European Journal of Biochemistry, 236(3), pp. 947-952

Lesley, S. A., Kuhn, P., Godzik, A., Deacon, A. M., Mathews, I., Kreusch, A., Spraggon, G., Klock, H. E., McMullan, D., Shin, T., Vincent, J., Robb, A., Brinen, L. S., Miller, M. D., McPhillips, T. M., Miller, M. A., Scheibe, D., Canaves, J. M., Guda, C., Jaroszewski, L., Selby, T. L., Elsliger, M.-A., Wooley, J., Taylor, S. S., Hodgson, K. O., Wilson, I. A., Schultz, P. G. and Stevens, R. C. (2002) 'Structural genomics of the Thermotoga maritima proteome implemented in a high-throughput structure determination pipeline', Proceedings of the National Academy of Sciences of the United States of America, 99(18), pp. 11664-11669

Liang, Y.-J. and Jiang, J.-G. (2015) 'Characterization of malic enzyme and the regulation of its activity and metabolic engineering on lipid production', RSC Advances, 5(56), pp. 45558-45570

Lin, R. C. and Davis, E. J. (1974) 'Malic enzymes of rabbit heart mitochondria. Separation and comparison of some characteristics of a nicotinamide adenine dinucleotidepreferring and a nicotinamide adenine dinucleotide phosphate-specific enzyme', Journal of Biological Chemistry, 249(12), pp. 3867-3875

Lobit, P., Genard, M., Soing, P. and Habib, R. (2006) 'Modelling malic acid accumulation in fruits: relationships with organic acids, potassium, and temperature', Journal of Experimental Botany, 57(6), pp. 1471-1483

López, R., Aznar, M., Cacho, J. and Ferreira, V. (2002) 'Determination of minor and trace volatile compounds in wine by solid-phase extraction and gas chromatography with mass spectrometric detection', Journal of Chromatography A, 966(1), pp. 167-177

Lupu, A., Compagnone, D. and Palleschi, G. (2004) 'Screen-printed enzyme electrodes for the detection of marker analytes during winemaking', Analytica Chimica Acta, 513(1), pp. 67-72 
Ma, B., Yuan, Y., Gao, M., Li, C., Ogutu, C., Li, M. and Ma, F. (2018) 'Determination of predominant organic acid components in Malus species: correlation with apple domestication', Metabolites, 8(4), pp. 74

Maines, A., Prodromidis, M. I., Tzouwara-Karayanni, S. M., Karayannis, M. I., Ashworth, D. and Vadgama, P. (2000) 'Reagentless enzyme electrode for malate based on modified polymeric membranes', Analytica Chimica Acta, 408(1), pp. 217-224

Manzoli, A., Tomita, I. N., Fertonani, F. L., de Oliveira-Neto, G., Mascini, M. and Yamanaka, H. (2004) 'Determination of malic acid in real samples by using enzyme immobilized reactors and amperometric detection', Analytical Letters, 37(9), pp. $1823-1832$

Matsue, T., Yamada, H., Chang, H.-C., Uchida, I., Nagata, K. and Tomita, K. (1990) 'Electron transferase activity of diaphorase (NADH:acceptor oxidoreductase) from Bacillus stearothermophilus', Biochimica et Biophysica Acta - Protein Structure and Molecular Enzymology, 1038(1), pp. 29-38

Matthews, C. J., Andrews, E. S. V. and Patrick, W. M. (2021) 'Enzyme-based amperometric biosensors for malic acid - A review', Analytica Chimica Acta, 1156, pp. 338218

Mazzei, F., Botrè, F. and Favero, G. (2007) 'Peroxidase based biosensors for the selective determination of D,L-lactic acid and L-malic acid in wines', Microchemical Journal, 87(1), pp. 81-86

Messia, M. C., Compagnone, D., Esti, M. and Palleschi, G. (1996) 'A bienzyme electrode probe for malate', Analytical Chemistry, 68(2), pp. 360-365

Miertuš, S., Katrlík, J., Pizzariello, A., Stred'anský, M., Švitel, J. and Švorc, J. (1998) 'Amperometric biosensors based on solid binding matrices applied in food quality monitoring', Biosensors and Bioelectronics, 13(7), pp. 911-923

Minárik, P., Tomášková, N., Kollárová, M. and Antalík, M. (2002) 'Malate dehydrogenases structure and function', General Physiology and Biophysics, 21(3), pp. 257-265

Mizutani, F., Yabuki, S. and Asai, M. (1991) 'L-Malate-sensing electrode based on malate dehydrogenase and NADH oxidase', Analytica Chimica Acta, 245, pp. 145-150

Moing, A., Rothan, C., Svanella, L., Just, D., Diakou, P., Raymond, P., Gaudillère, J.-P. and Monet, R. (2000) 'Role of phosphoenolpyruvate carboxylase in organic acid accumulation during peach fruit development', Physiologia Plantarum, 108(1), pp. 110

Molenaar, D., van der Rest, M. E. and Petrović, S. (1998) 'Biochemical and genetic characterization of the membrane-associated malate dehydrogenase (acceptor) from Corynebacterium glutamicum', European Journal of Biochemistry, 254(2), pp. 395403

Molinero-Abad, B., Alonso-Lomillo, M. A., Domínguez-Renedo, O. and Arcos-Martínez, M. J. (2014) 'Malate quinone oxidoreductase biosensors based on tetrathiafulvalene and gold nanoparticles modified screen-printed carbon electrodes for malic acid determination in wine', Sensors and Actuators B: Chemical, 202, pp. 971-975 
Monošík, R., Stredanský, M., Greif, G. and Šturdík, E. (2012a) 'Comparison of biosensors based on gold and nanocomposite electrodes for monitoring of malic acid in wine', Central European Journal of Chemistry, 10(1), pp. 157-164

Monošík, R., Ukropcova, D., Stredansky, M. and Šturdik, E. (2012b) 'Multienzymatic amperometric biosensor based on gold and nanocomposite planar electrodes for glycerol determination in wine', Analytical Biochemistry, 421(1), pp. 256-261

Morrison, J. C. and Noble, A. C. (1990) 'The Effects of Leaf and Cluster Shading on the Composition of Cabernet Sauvignon Grapes and on Fruit and Wine Sensory Properties', American Journal of Enology and Viticulture, 41(3), pp. 193-200

Mundaca-Uribe, R., Valencia-Muñoz, D., Castillo, R. C., Hinojosa, J. N., Bustos, M. A. and Peña-Farfal, C. (2017) 'Bienzymatic biosensor for malic acid based on malate dehydrogenase and transaminase immobilized onto a glassy carbon powder/carbon nanotubes/NAD ${ }^{+}$composite electrode', Electroanalysis, 29(1), pp. 238-243

Nagels, L. J. and Staes, E. (2001) 'Polymer (bio)materials design for amperometric detection in LC and FIA', Trends in Analytical Chemistry, 20(4), pp. 178-185

Nguyen, H. H., Lee, S. H., Lee, U. J., Fermin, C. D. and Kim, M. (2019) 'Immobilized enzymes in biosensor applications', Materials, 12(1), pp. 121

Nikolaou, C., Karabagias, I. K., Gatzias, I., Kontakos, S., Badeka, A. and Kontominas, M. G. (2017) 'Differentiation of fresh Greek orange juice of the Merlin cultivar according to geographical origin based on the combination of organic acid and sugar content as well as physicochemical parameters using chemometrics', Food Analytical Methods, 10(7), pp. 2217-2228

Nishiyama, M., Birktoft, J. J. and Beppu, T. (1993) 'Alteration of coenzyme specificity of malate dehydrogenase from Thermus flavus by site-directed mutagenesis', Journal of Biological Chemistry, 268, pp. 4656-4660

Nishiyama, M., Matsubara, N., Yamamoto, K., Iijima, S., Uozumi, T. and Beppu, T. (1986) 'Nucleotide sequence of the malate dehydrogenase gene of Thermus flavus and its mutation directing an increase in enzyme activity', Journal of Biological Chemistry, 261(30), pp. 14178-14183

Nyambe-Silavwe, H., Villa-Rodriguez, J. A., Ifie, I., Holmes, M., Aydin, E., Jensen, J. M. and Williamson, G. (2015) 'Inhibition of human $\alpha$-amylase by dietary polyphenols', Journal of Functional Foods, 19, pp. 723-732

Omaye, S. T., David Turnbull, J. and Sauberlich, H. E. (1979) 'Selected methods for the determination of ascorbic acid in animal cells, tissues, and fluids', Methods in Enzymology: Academic Press, pp. 3-11.

Ough, C. S. (1964) 'Fermentation rates of Grape Juice. I. Effects of Temperature and Composition on White Juice Fermentation Rates', American Journal of Enology and Viticulture, 15(4), pp. 167 
Pairoba, C. F., Colombo, S. L. and Andreo, C. S. (1996) 'Flavonoids as inhibitors of NADPmalic enzyme and PEP carboxylase from C4 plants', Bioscience, Biotechnology, and Biochemistry, 60(5), pp. 779-783

Palleschi, G., Volpe, G., Compagnone, D., La Notte, E. and Esti, M. (1994) 'Bioelectrochemical determination of lactic and malic acids in wine', Talanta, 41(6), pp. $917-923$

Palma, M. and Barroso, C. G. (2002) 'Ultrasound-assisted extraction and determination of tartaric and malic acids from grapes and winemaking by-products', Analytica Chimica Acta, 458(1), pp. 119-130

Park, S. H., Kiick, D. M., Harris, B. G. and Cook, P. F. (1984) 'Kinetic mechanism in the direction of oxidative decarboxylation for NAD-malic enzyme from Ascaris suum', Biochemistry, 23(23), pp. 5446-5453

Prieto-Simón, B. and Fàbregas, E. (2004) 'Comparative study of electron mediators used in the electrochemical oxidation of NADH', Biosensors and Bioelectronics, 19(10), pp. $1131-1138$

Prodromidis, M. I. and Karayannis, M. I. (2002) 'Enzyme based amperometric biosensors for food analysis', Electroanalysis, 14(4), pp. 241-261

Prodromidis, M. I., Tzouwara-Karayanni, S. M., Karayannis, M. I., Vadgama, P. and Maines, A. (1996) 'Amperometric determination of L-malic acid in a flow injection analysis manifold using packed-bed enzyme reactors', Analyst, 121(4), pp. 435-439

Rahman, M. M., Ahammad, A. J. S., Jin, J.-H., Ahn, S. J. and Lee, J.-J. (2010) 'A comprehensive review of glucose biosensors based on nanostructured metal-oxides', Sensors, 10(5), pp. 4855-4886

Rao, G. S. J., Coleman, D. E., Karsten, W. E., Cook, P. F. and Harris, B. G. (2003) 'Crystallographic Studies on Ascaris suum NAD-Malic Enzyme Bound to Reduced Cofactor and Identification of an Effector Site', Journal of Biological Chemistry, 278(39), pp. 38051-38058

Rathee, K., Dhull, V., Dhull, R. and Singh, S. (2016) 'Biosensors based on electrochemical lactate detection: a comprehensive review', Biochemistry and Biophysics Reports, 5, pp. 35-54

Renedo, O. D., Alonso-Lomillo, M. A. and Martínez, M. J. A. (2007) 'Recent developments in the field of screen-printed electrodes and their related applications', Talanta, 73(2), pp. 202-219

Reyes-De-Corcuera, J. I., Olstad, H. E. and García-Torres, R. (2018) 'Stability and stabilization of enzyme biosensors: the key to successful application and commercialization', Annual Review of Food Science and Technology, 9(1), pp. 293322

Ribéreau-Gayon, P., Dubourdieu, D., Donèche, B. and Lonvaud, A. (2006) Handbook of enology, Volume 1: The microbiology of wine and vinifications. John Wiley \& Sons. 
Röhlen, D. L., Pilas, J., Schöning, M. J. and Selmer, T. (2017) 'Development of an amperometric biosensor platform for the combined determination of L-malic, fumaric, and L-aspartic acid', Applied Biochemistry and Biotechnology, 183(2), pp. 566-581

Sáenz-Navajas, M.-P., Avizcuri, J.-M., Ballester, J., Fernández-Zurbano, P., Ferreira, V., Peyron, D. and Valentin, D. (2015) 'Sensory-active compounds influencing wine experts' and consumers' perception of red wine intrinsic quality', LWT - Food Science and Technology, 60(1), pp. 400-411

Sassolas, A., Blum, L. J. and Leca-Bouvier, B. D. (2012) 'Immobilization strategies to develop enzymatic biosensors', Biotechnology Advances, 30(3), pp. 489-511

Sato, I., Yoshikawa, J., Furusawa, A., Chiku, K., Amachi, S. and Fujii, T. (2010) 'Isolation and properties of malic enzyme and its gene in Rhodopseudomonas palustris No. 7', Bioscience, Biotechnology, and Biochemistry, 74(1), pp. 75-81

Sereni, A., Phan, Q., Osborne, J. and Tomasino, E. (2020) 'Impact of the Timing and Temperature of Malolactic Fermentation on the Aroma Composition and Mouthfeel Properties of Chardonnay Wine', Foods (Basel, Switzerland), 9(6), pp. 802

Shekhar, V. C. and Iritani, W. M. (1979) 'Changes in malic and citric acid contents during growth and storage of Solanum tuberosum L', American potato journal, 56(2), pp. 8794

Silber, A., Bräuchle, C. and Hampp, N. (1994) 'Dehydrogenase-based thick-film biosensors for lactate and malate', Sensors and Actuators B: Chemical, 18(1), pp. 235-239

Silverstein, T. P. and Goodney, D. E. (2010) 'Enzyme-linked biosensors: Michaelis-Menten kinetics need not apply', Journal of Chemical Education, 87(9), pp. 905-907

Situmorang, M., Gooding, J. J., Hibbert, D. B. and Barnett, D. (2001) 'Development of potentiometric biosensors using electrodeposited polytyramine as the enzyme immobilization matrix', Electroanalysis, 13(18), pp. 1469-1474

Sochorova, L., Torokova, L., Baron, M. and Sochor, J. (2018) 'Electrochemical and others techniques for the determination of malic acid and tartaric acid in must and wine', International Journal of Electrochemical Science, 13(9), pp. 9145-9165

Soleymani, L. and Li, F. (2017) 'Mechanistic challenges and advantages of biosensor miniaturization into the nanoscale', ACS Sensors, 2(4), pp. 458-467

Soyer, Y., Koca, N. and Karadeniz, F. (2003) 'Organic acid profile of Turkish white grapes and grape juices', Journal of Food Composition and Analysis, 16(5), pp. 629-636

Su, K.-L., Chang, K.-Y. and Hung, H.-C. (2009) 'Effects of structural analogues of the substrate and allosteric regulator of the human mitochondrial NAD $(\mathrm{P})$ +-dependent malic enzyme', Bioorganic \& Medicinal Chemistry, 17(15), pp. 5414-5419

Thévenot, D. R., Toth, K., Durst, R. A. and Wilson, G. S. (2001) 'Electrochemical biosensors: recommended definitions and classification', Biosensors and Bioelectronics, 16(1), pp. 121-131 
Tonomura, B. i., Nakatani, H., Ohnishi, M., Yamaguchi-Ito, J. and Hiromi, K. (1978) 'Test reactions for a stopped-flow apparatus: Reduction of 2,6-dichlorophenolindophenol and potassium ferricyanide by l-ascorbic acid', Analytical Biochemistry, 84(2), pp. $370-383$

Turner, A. P. (2013) 'Biosensors: sense and sensibility', Chemical Society Reviews, 42(8), pp. 3184-3196

Ucar, D., Zhang, Y. and Angelidaki, I. (2017) 'An overview of electron acceptors in microbial fuel cells', Frontiers in Microbiology, 8, pp. 643-643

Urbansky, E. T. and Schock, M. R. (2000) 'Understanding, Deriving, and Computing Buffer Capacity', Journal of Chemical Education, 77(12), pp. 1640

Vagenende, V., Yap, M. G. and Trout, B. L. (2009) 'Mechanisms of protein stabilization and prevention of protein aggregation by glycerol', Biochemistry, 48(46), pp. 1108411096

Vallée-Bélisle, A., Ricci, F. and Plaxco, K. W. (2012) 'Engineering biosensors with extended, narrowed, or arbitrarily edited dynamic range', Journal of the American Chemical Society, 134(6), pp. 2876-2879

Vargas, E., Ruiz, M. A., Ferrero, F. J., Campuzano, S., Ruiz-Valdepeñas Montiel, V., Reviejo, A. J. and Pingarrón, J. M. (2016) 'Automatic bionalyzer using an integrated amperometric biosensor for the determination of L-malic acid in wines', Talanta, 158, pp. 6-13

Vashishtha, A. K. and Konigsberg, W. H. (2016) 'Effect of Different Divalent Cations on the Kinetics and Fidelity of RB69 DNA Polymerase', Biochemistry, 55(18), pp. 26612670

Vasylieva, N. and Marinesco, S. (2013) 'Enzyme Immobilization on Microelectrode Biosensors', in Marinesco, S. \& Dale, N. (eds.) Microelectrode Biosensors. Totowa, NJ: Humana Press, pp. 95-114.

Veskoukis, A. S., Margaritelis, N. V., Kyparos, A., Paschalis, V. and Nikolaidis, M. G. (2018) 'Spectrophotometric assays for measuring redox biomarkers in blood and tissues: the NADPH network', Redox Report, 23(1), pp. 47-56

Volschenk, H., van Vuuren, H. J. J. and Viljoen-Bloom, M. (2006) 'Malic acid in wine: origin, function and metabolism during vinification', South African Journal for Enology and Viticulture, 27(2), pp. 123-136

Wang, Z. D., Wang, B. J., Ge, Y. D., Pan, W., Wang, J., Xu, L., Liu, A. M. and Zhu, G. P. (2011) 'Expression and identification of a thermostable malate dehydrogenase from multicellular prokaryote Streptomyces avermitilis MA-4680', Molecular Biology Reports, 38(3), pp. 1629-1636

Willeford, K. O. and Wedding, R. T. (1987) 'pH Effects on the Activity and Regulation of the NAD Malic Enzyme', Plant Physiology, 84(4), pp. 1084-1087 
Wittig, U., Kania, R., Golebiewski, M., Rey, M., Shi, L., Jong, L., Algaa, E., Weidemann, A., Sauer-Danzwith, H., Mir, S., Krebs, O., Bittkowski, M., Wetsch, E., Rojas, I. and Müller, W. (2012) 'SABIO-RK--database for biochemical reaction kinetics', Nucleic acids research, 40(Database issue), pp. D790-D796

Wolosiuk, R. A., Buchanan, B. B. and Crawford, N. A. (1977) 'Regulation of NADP-malate dehydrogenase by the light-actuated ferredoxin/thioredoxin system of chloroplasts', FEBS Letters, 81(2), pp. 253-258

Yéramian, N., Chaya, C. and Suárez Lepe, J. A. (2007) 'L-(-)-malic acid production by Saccharomyces spp. during the alcoholic fermentation of wine', Journal of Agricultural and Food Chemistry, 55(3), pp. 912-919

Yoshioka, S., Ukeda, H., Matsumoto, K. and Osajima, Y. (1992) 'Simultaneous flow injection analysis of L-lactate and L-malate in wine based on the use of enzyme reactors', Electroanalysis, 4(5), pp. 545-548

Zhang, H., Zhang, L., Chen, H., Chen, Y. Q., Ratledge, C., Song, Y. and Chen, W. (2013) 'Regulatory properties of malic enzyme in the oleaginous yeast, Yarrowia lipolytica, and its non-involvement in lipid accumulation', Biotechnology Letters, 35(12), pp. 2091-2098

Zhang, Y., Aono, T., Poole, P. and Finan, T. M. (2012) 'NAD(P)+-malic enzyme mutants of Sinorhizobium sp. strain NGR234, but not Azorhizobium caulinodans ORS571, maintain symbiotic N2 fixation capabilities', Applied and environmental microbiology, 78(8), pp. 2803-2812

Zoecklein, B., Fugelsang, K. C., Gump, B. H. and Nury, F. S. (1999) Wine Analysis and Production. New York: Springer.

Zolghadri, S., Bahrami, A., Hassan Khan, M. T., Munoz-Munoz, J., Garcia-Molina, F., Garcia-Canovas, F. and Saboury, A. A. (2019) 'A comprehensive review on tyrosinase inhibitors', Journal of Enzyme Inhibition and Medicinal Chemistry, 34(1), pp. 279-309 


\section{Chapter Eight - Appendices}

Appendix 1. Chromatograms produced during sizeexclusion chromatography for protein purification
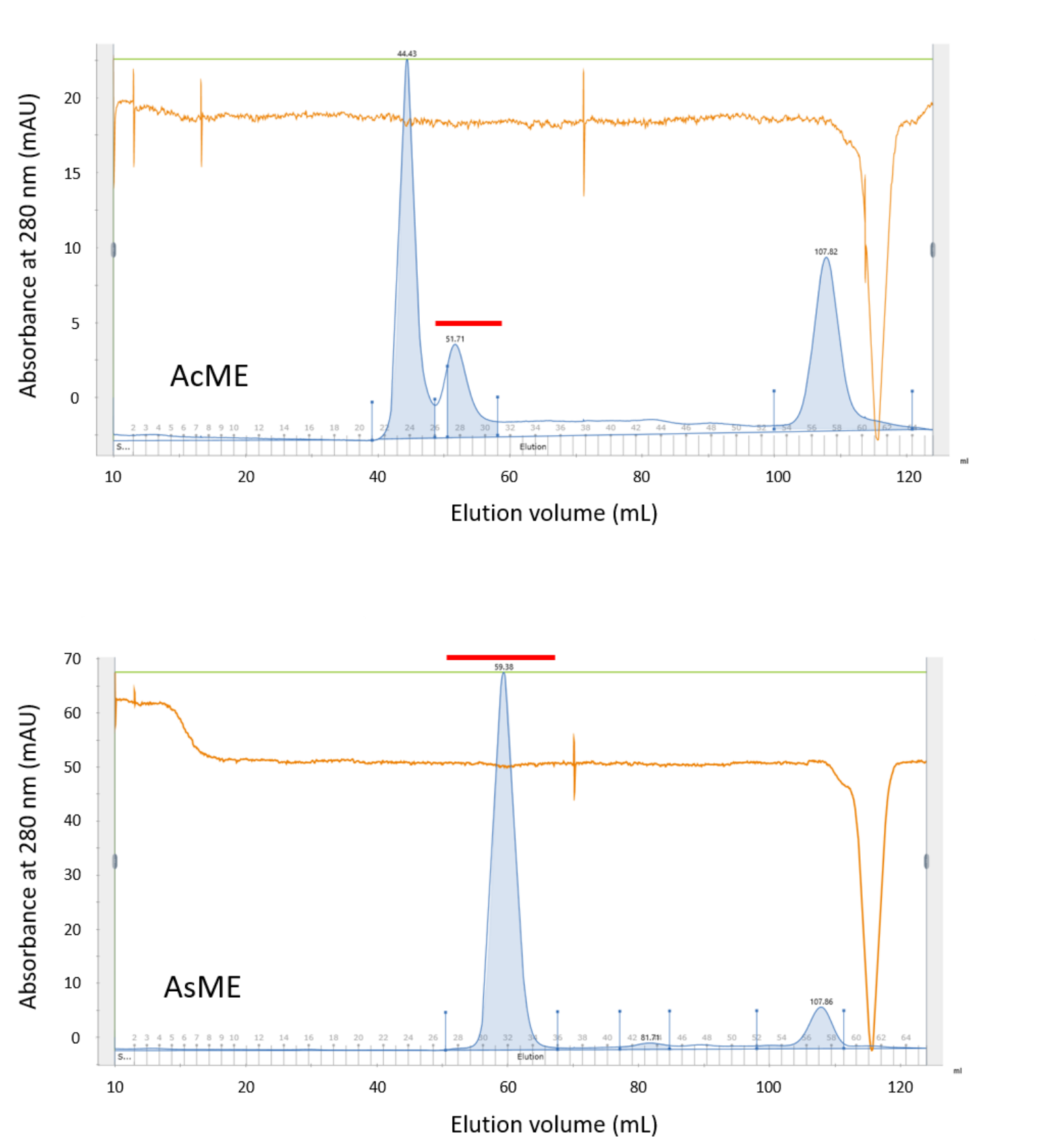


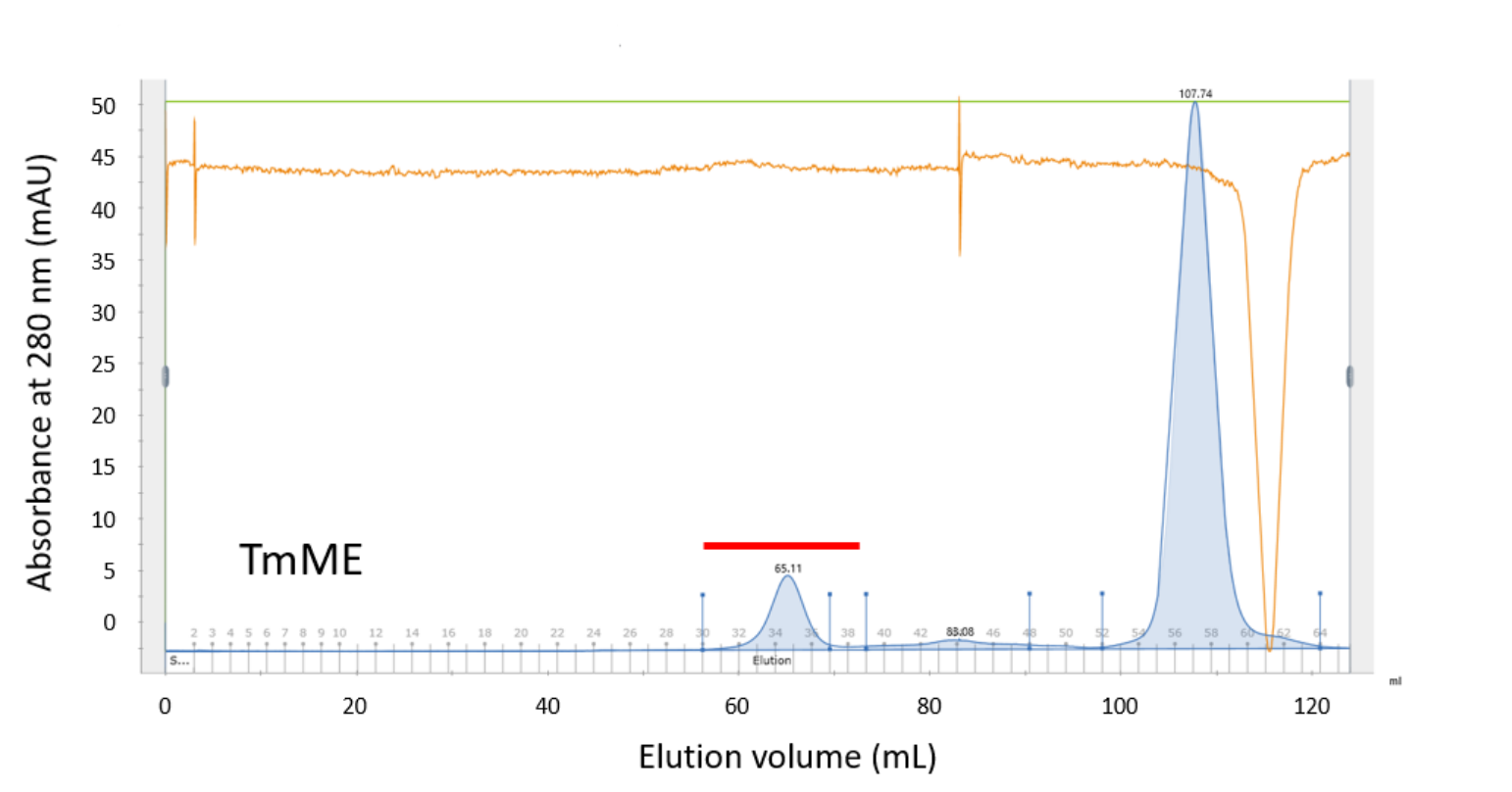

Supplementary figure 1. Size exclusion chromatography chromatograms for AcME, AsME and TmME. The change in UV absorbance at $280 \mathrm{~nm}$ is plotted against the volume eluted from the column (integrated blue trace). Red bars represent the fractions run on SDS-PAGE gels to check the protein size and purity.

\section{Appendix 2. NAD+ optimization for $\operatorname{AsME(R181Q)~kinetics~}$ assays when using $25 \mathrm{mM} \mathrm{Mg}^{2+}$}

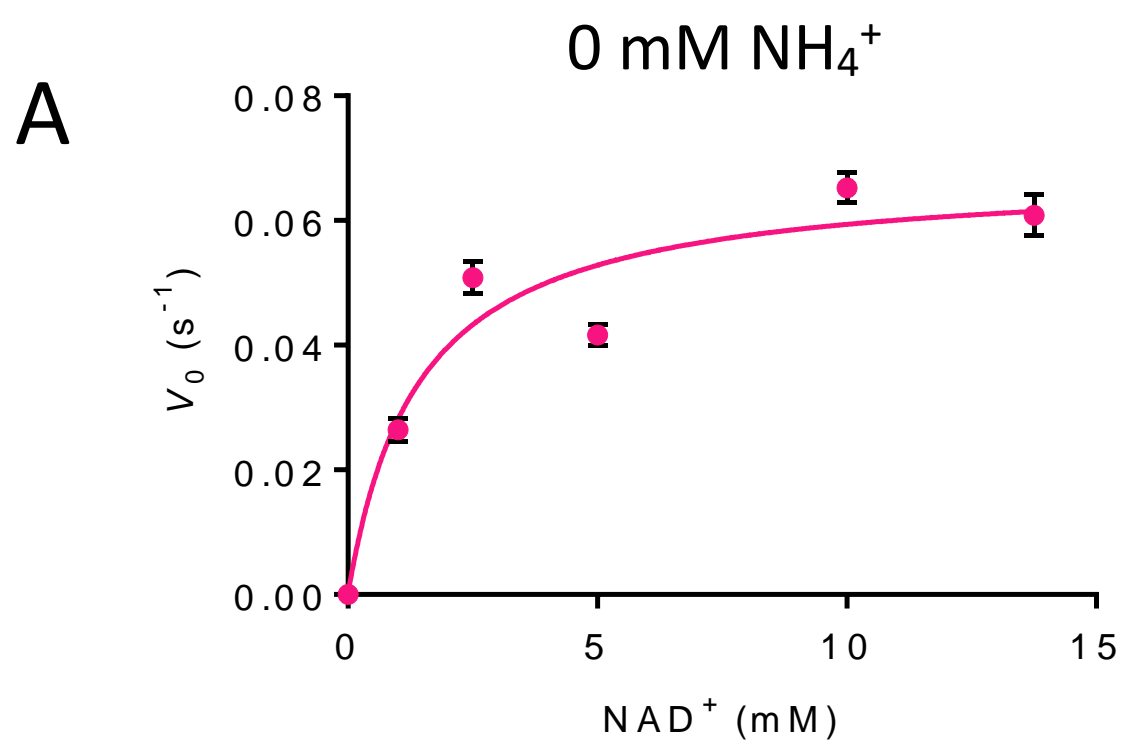



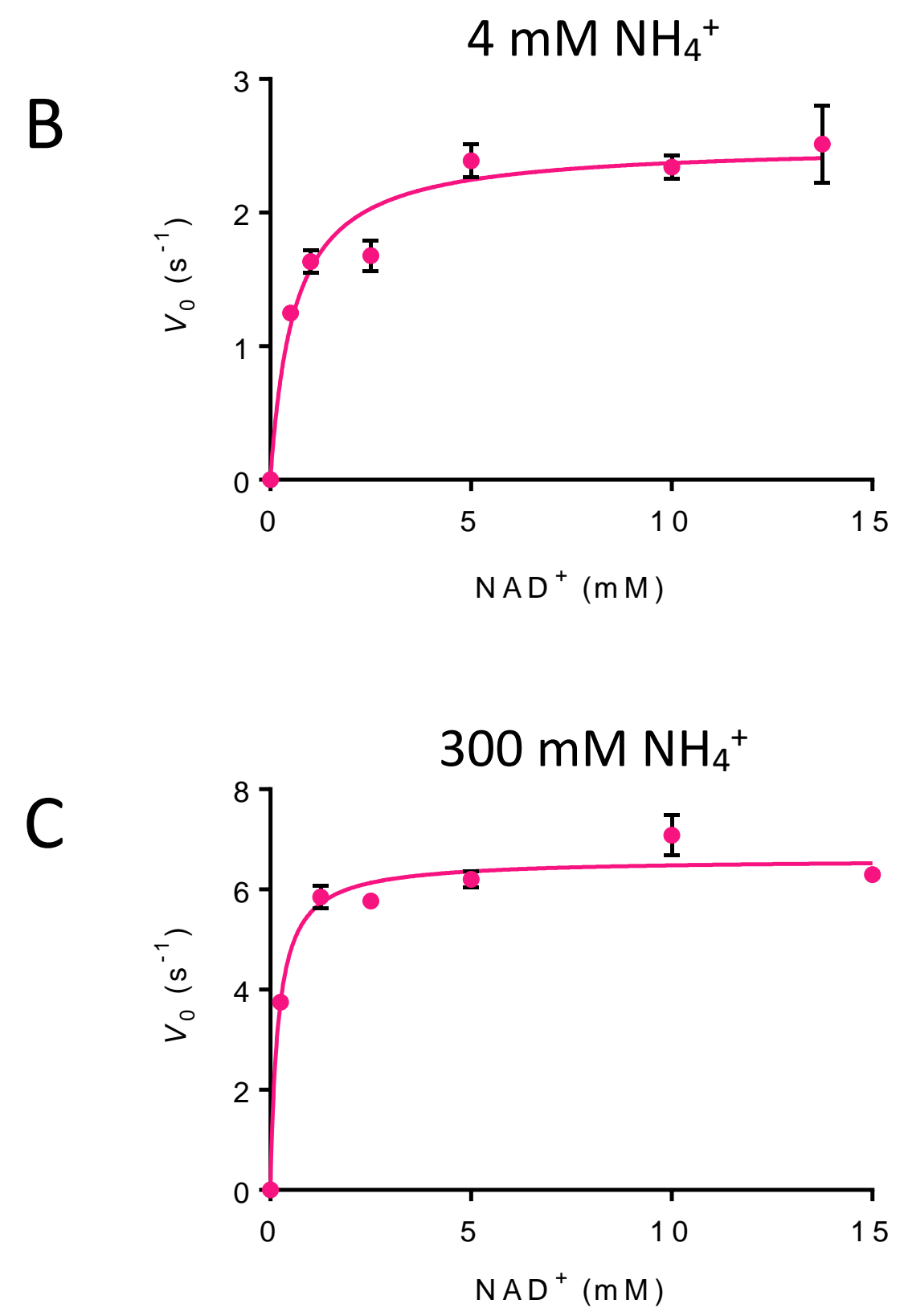

Supplementary figure 2. The kinetic behaviour of AsME(R181Q) at different concentrations of $N A D^{+}$, when using $25 \mathrm{mM} \mathrm{Mg}^{2+}$ in assays. The initial velocity $\left(V_{0}\right)$ is plotted against increasing concentrations of $\mathrm{NAD}^{+}$when using (A) $0 \mathrm{mM}$, (B) $4 \mathrm{mM}$ and (C) $300 \mathrm{mM} \mathrm{NH}_{4}{ }^{+}$in assays. Assays used a fixed concentration of $100 \mathrm{mM}$ malate. Plots have been fitted with the Michaelis-Menten equation, with each point representing the mean of triplicates. Error bars showing the standard error, though are sometimes too small to be seen. 